\title{
Development of a Sorption-based Joule-Thomson Cooler for the METIS INSTRUMent ON E-ELT
}




\title{
Development of a Sorption-based Joule-Thomson Cooler for the METIS Instrument on E-ELT
}

\author{
Yingzhe Wu
}




\section{Ph.D. committee:}

\section{Chairman}

prof. dr. ir. J.W.M. Hilgenkamp University of Twente

\section{Promoter}

prof. dr. ir. H.J.M. ter Brake University of Twente

\section{Members}

prof. dr. B.R. Brandl

Leiden University

prof. dr. J.G.E. Gardeniers

University of Twente

prof. dr. J.L. Herek

University of Twente

prof. dr. ir. T.H. van der Meer

University of Twente

dr. ir. S. Vanapalli

University of Twente

prof. dr. A.T.A.M. de Waele

Eindhoven University of Technology

Frontcover: A helium sorption-compressor cell for the METIS cooler. The honeycomb pattern on the background is the E-ELT's 40-metre primary mirror that consists of 798 hexagonal segments (Source: ESO).

Backcover: An Artist's impression of the E-ELT (Source: ESO). The background picture taken from the Atacama Desert where the E-ELT will be located, is the centre of Milky Way. (Source: ESO/G. Hüdepohl, atacamaphoto.com)

The research described in this thesis was enabled through the Netherlands Research School for Astronomy (NOVA) by financial support from the Netherlands Organization for Scientific Research (NWO) under contract 184.021.006. It was carried out at the Energy, Materials and Systems group of the Faculty of Science and Technology of the University of Twente, with collabration Airbus Defence and Space Netherlands (formerly Dutch Space).

Development of a sorption-based Joule-Thomson cooler for the METIS instrument on E-ELT Yingzhe Wu

Ph.D thesis, University of Twente, Enschede, the Netherlands

ISBN: 978-90-365-3993-7

DOI: $10.3990 / 1.9789036539937$

Printed by PrintPartners Ipskamp, Enschede, the Netherlands

(C) Yingzhe Wu, Nov. 2015 


\title{
Development of a Sorption-based Joule-Thomson Cooler for the METIS Instrument on E-ELT
}

\author{
PROEFSCHRIFT
}

ter verkrijging van

de graad van doctor aan de Universiteit Twente, op gezag van de rector magnificus,

prof. dr. H. Brinksma,

volgens besluit van het College voor Promoties

in het openbaar te verdedigen

op woensdag 25 november 2015, om 16.45 uur

door

\section{Yingzhe Wu}

geboren op 24 april, 1985

te Hangzhou, CHINA 
Dit proefschrift is goedgekeurd door de promotor:

prof. dr. ir. H.J.M. ter Brake 
To my beloved wife, Yi and my parents 



\section{Latin letters}

$\begin{array}{ll}A & \text { Area } \\ A_{c} & \text { Cross sectional area } \\ b & \text { van de Waals volume } \\ c & \text { Specific heat capacity (solid) } \\ c_{p} & \text { Specific heat capacity at the constant pressure } \\ c_{p} & \text { Specific heat capacity at the constant volume } \\ C & \text { Heat capacity } \\ D & \text { Diameter } \\ E & \text { Effectiveness of heat exchanger } \\ F & \text { View factor } \\ f_{D} & \text { Darcy-Weisbach friction factor } \\ f_{s} & \text { Safety factor } \\ f_{w} & \text { Welding factor } \\ g & \text { Specific Gibbs free energy } \\ G & \text { Gibbs free energy } \\ h & \text { Specific enthalpy } \\ \dot{H} & \text { Enthalpy flow rate } \\ k & \text { Thermal conductivity } \\ L_{b} & \text { Anchor point coordinate of the thermal link } \\ L_{t o t} & \text { Total length of the sorption compressor } \\ m & \text { Mass } \\ \dot{m} & \text { Mass-flow rate } \\ M & \text { Molar mass }\end{array}$

$\left[\mathrm{m}^{2}\right]$

$\left[\mathrm{m}^{2}\right]$

$\left[\mathrm{m}^{3} \mathrm{~mol}^{-1}\right.$ ]

[ $\mathrm{J} \mathrm{kg}^{-1} \mathrm{~K}^{-1}$ ]

[ $\mathrm{J} \mathrm{kg}^{-1} \mathrm{~K}^{-1}$ ]

$\left[\mathrm{J} \mathrm{kg}^{-1} \mathrm{~K}^{-1}\right.$ ]

[ $\mathrm{J} \mathrm{K}^{-1}$ ]

[m]

[-]

[-]

[-]

[-]

[-]

[ $\mathrm{J} \mathrm{kg}^{-1}$ ]

[J]

[ $\mathrm{J} \mathrm{kg}^{-1}$ ]

[ $\mathrm{W} \mathrm{kg}^{-1}$ ]

[W m ${ }^{-1} \mathrm{~K}^{-1}$ ]

[m]

[m]

[kg]

[ $\mathrm{kg} \mathrm{s}^{-1}$ ]

$\left[\mathrm{kg} \mathrm{mol}^{-1}\right.$ ] 
$n$

$p$

$P_{L}$

$Q$

$\dot{Q}$

$\dot{q}$

$r$

$R$

$R$

$s$

$S$

$t$

$T$

$U$

V

$\vec{V}$

$\dot{W}$

$x$
Number of sorption compressor cells

Pressure

Heating power per length

Heat

Heat-flow rate (Power)

Heat normalized to the adsorbent mass

Radius

Thermal resistance

Universal gas constant

Specific entropy

Source term in the energy conservation equation

Time

Temperature

Heat transfer coefficient

Volume

Velocity vector

Power

Amount normalized to the adsorbent mass
$[-]$

$[\mathrm{Pa}]$

[W m ${ }^{-1}$ ]

$[\mathrm{J}]$

[W]

[ $\mathrm{J} \mathrm{kg}^{-1}$ ]

[m]

$\left[\mathrm{K} \mathrm{W}^{-1}\right]$

[ $\mathrm{J} \mathrm{K}^{-1} \mathrm{~mol}^{-1}$ ]

[ $\mathrm{J} \mathrm{kg}^{-1} \mathrm{~K}^{-1}$ ]

[W m ${ }^{-3}$ ]

[s]

[K or $\left.{ }^{\circ} \mathrm{C}\right]$

[W m ${ }^{-2} \mathrm{~K}^{-1}$ ]

$\left[\mathrm{m}^{3}\right]$

$\left[\mathrm{m} \mathrm{s}^{-1}\right]$

[W]

[W]

\section{Greek letters}

$\begin{array}{ll}\alpha & \text { Porosity } \\ \delta & \text { Thickness } \\ \epsilon & \text { Emissivity } \\ \rho & \text { Density } \\ \sigma_{y} & \text { Yield strength } \\ \Omega & \text { Thermal expansion of the superheated liquid }\end{array}$

$[-]$

[m]

[-]

$\left[\mathrm{kg} \mathrm{m}^{-3}\right]$

[Pa]

$\left[\mathrm{K}^{-1}\right]$

\section{Accents and subscripts}

$\begin{array}{llll} & \text { Amount in a compressor cycle } & \text { cd } & \text { Cooling down } \\ 1 s t G M & \text { First stage of the GM cooler } & \text { cell } & \text { Sorption compressor cell } \\ 2 n d G M & \text { Second stage of the GM cooler } & \text { cond } & \text { Thermal conduction } \\ a c & \text { Aftercooling } & \text { compr } & \text { Compressor } \\ a d d & \text { Additional } & c r & \text { Critical } \\ a d s & \text { Adsorbed } & c y c & \text { Compression cycle } \\ a m b & \text { Ambient } & \text { CONT } & \text { Container } \\ b & \text { Bottom } & e f f & \text { Effective } \\ b & \text { Bulk (density) } & \text { ex } & \text { Exhausting } \\ B F & \text { Buffer } & \text { Evap } & \text { Evaporator } \\ B F H & \text { High pressure buffer } & e x & \text { Exhausting } \\ B F L & \text { Low pressure buffer } & f & \text { Final } \\ c & \text { Cooling } & f r e & \text { Free working fluid } \\ c & \text { Cold } & h & \text { High pressure }\end{array}$




$\begin{array}{llll}h p & \text { High pressure } & \text { ot } & \text { Outer tube } \\ H S & \text { Heat-sink } & \text { out } & \text { Out-flow } \\ H T & \text { Heater } & \text { OA } & \text { Overall } \\ i & \text { Inner } & \text { para } & \text { Parasitic } \\ \text { im } & \text { Intermediate pressure } & \text { preC } & \text { Pre-cooling } \\ \text { in } & \text { In-flow } & \text { ref } & \text { Reference } \\ \text { input } & \text { Input power } & \text { sor } & \text { Adsorbent } \\ i t & \text { Inner tube } & \text { sorp } & \text { Sorption } \\ I S L & \text { Insulation layer } & s v & \text { Saturated vapor } \\ l & \text { Low pressure } & \text { SC } & \text { Sorption compressor } \\ l p & \text { Low pressure } & s s & \text { Single stage compressor } \\ n b & \text { Normal boiling point } & \text { rad } & \text { Thermal radiation } \\ n b, l & \text { Liquid at normal boiling point } & \text { tot } & \text { Total } \\ n e t & \text { Net value } & t & \text { True (density) } \\ n s & \text { N-stage compressor } & \text { tr } & \text { Transferred } \\ o & \text { Outer } & \text { void } & \text { Void volume } \\ \text { opt } & \text { Optimized } & w & \text { Warm }\end{array}$

\section{Abbreviations}

$\begin{array}{ll}\text { ADSN } & \text { Airbus Defence and Space Netherlands } \\ \text { BSC } & \text { Baseline sorption cell } \\ \text { BV } & \text { Bypass valve } \\ \text { CFHX } & \text { Counter flow heat exchanger } \\ \text { CHX } & \text { Cold heat exchanger } \\ \text { COP } & \text { Coefficient of performance } \\ \text { CV } & \text { Control volume } \\ \text { DPLH } & \text { Dual-pressure Linde-Hampson cycle } \\ \text { E-ELT } & \text { European Extremely Large Telescope } \\ \text { Eff } & \text { Effectiveness of heat exchanger } \\ \text { ESA } & \text { European Space Agency } \\ \text { ESO } & \text { European Southern Observatory } \\ \text { GGHS } & \text { Gas-gap heat switch } \\ \text { GM } & \text { Gifford-McMahon } \\ \text { HX } & \text { Heat exchanger } \\ \text { JPL } & \text { NASA's Jet Propulsion Laboratory } \\ \text { JT } & \text { Joule-Thomson } \\ \text { JTCS } & \text { Joule-Thomson cold stage } \\ \text { LVT } & \text { Very Large Telescope } \\ \text { METIS } & \text { Mid-infrared E-ELT Imager and Spectrograph } \\ \text { MLI } & \text { Multi-layer insulation } \\ \text { preCHX } & \text { Pre-cooling heat exchanger } \\ \text { RCEC } & \text { Radial-conductance-enhanced cell } \\ \text { SC } & \text { Sorption compressor } \\ \end{array}$





\section{Contents}

1 Introduction 1

1.1 E-ELT \& METIS instrument . . . . . . . . . . . . . . . 2

1.2 Sorption cooler $\&$ its history . . . . . . . . . . . . . . 4

1.3 Motivation $\&$ research roadmap . . . . . . . . . . . . 6

1.4 Outline .......................... 7

2 Fundamentals of Joule-Thomson Cold Stage and Sorption Compressor 9

2.1 Joule-Thomson cold stage . . . . . . . . . . . . . . . . . 10

2.1.1 Basic Joule-Thomson cold stage: Linde-Hampson system . . . . 10

2.1.2 Thermodynamic analysis for a basic Joule-Thomson cold stage . . 11

2.1.3 Precooled Joule-Thomson cold stage . . . . . . . . . . . . . 13

2.2 Sorption compressor . . . . . . . . . . . . . . . 15

2.2.1 Compression cycle in a sorption compressor . . . . . . . . . . 15

2.2.2 Fundamentals of the adsorption phenomenon . . . . . . . . . 18

2.2.3 Thermodynamic analysis of a sorption compressor . . . . . . . 20

2.3 Working fluid optimization for the sorption-based Joule-Thomson cooler . 24

2.3.1 Optimization criteria . . . . . . . . . . . . . . . 24

2.3.2 Optimization method ................ 24

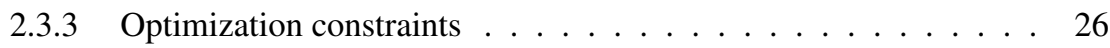

2.3.4 Discussion on optimization results . . . . . . . . . . . 27

2.4 Conclusions . . . . . . . . . . . . . . . . . . 35 
3 Baseline Design of the METIS Cooler 37

3.1 METIS cooler chain . . . . . . . . . . . . . . . . . 38

3.2 Baseline compressor configuration . . . . . . . . . . . . . . . 39

3.3 Baseline design . . . . . . . . . . . . . . . . . . 40

3.3.1 Sorption cooler modeling overview . . . . . . . . . . . . 40

3.3.2 Optimization of the operating parameters . . . . . . . . . 42

3.4 Demonstration plan for the METIS cooler . . . . . . . . . . . . 52

3.5 Conclusions . . . . . . . . . . . . . . . . . 55

4 Joule-Thomson Cold Stage Design and Demonstration for the METIS Cooler 57

4.1 Joule-Thomson cold stage configuration . . . . . . . . . . . . . 58

4.1.1 Parallel configuration vs serial configuration . . . . . . . . . 58

4.1.2 Alternative hydrogen-stage configurations . . . . . . . . . . . 59

4.2 Required effectiveness of the CFHXs in METIS cooler chain (baseline) . 62

4.3 CFHX design . . . . . . . . . . . . . . . . . . . . 63

4.3.1 Select the CFHX type . . . . . . . . . . . . . 63

4.3 .2 CFHX Modelling . . . . . . . . . . . . . . . . . . . 64

4.4 Full scale, $8 \mathrm{~K}$ helium Joule-Thomson cold stage . . . . . . . . . . . . . 67

4.4.1 An outlook of the experiment setup . . . . . . . . . . . 67

4.4.2 Precooling subsystem ............... . 70

$4.4 .3 \quad 15-8$ K CFHX . . . . . . . . . . . . . . . . . . 70

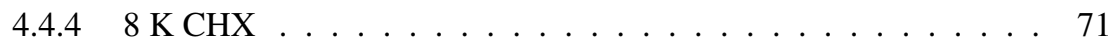

4.4.5 Joule-Thomson restriction . . . . . . . . . . . . 71

4.4.6 Experimental results and discussion . . . . . . . . . 72

4.5 Conclusions . . . . . . . . . . . . . . . . . . . . 74

5 Sorption Compressor Design for the METIS Cooler 77

5.1 Limitations of the gas-gap heat switch . . . . . . . . . . . . 78

5.2 Switchless configuration . . . . . . . . . . . . 78

5.3 Dynamic model . . . . . . . . . . . . . . . . . . . . . 79

5.3.1 Analytical description . . . . . . . . . . . . . . 79

5.3.2 Simulations and discussions . . . . . . . . . . . 82

5.4 Experimental validation of the dynamic model . . . . . . . . . . . . 85

5.4.1 Experimental setup . . . . . . . . . . . . . . 85

5.4.2 Experimental results and discussion . . . . . . . . . . 87

5.5 Performance comparison between the switchless and GGHS configurations 91

5.6 Detailed design of the sorption compressor cells for the METIS cooler . . 93 


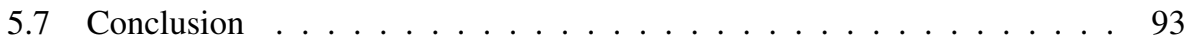

6 Scaled Helium Sorption Compressor $\quad 95$

6.1 Overview of the experimental setup . . . . . . . . . . . . 96

6.2 Detailed design . . . . . . . . . . . . . . . . . . . . . . 98

6.2 .1 Sorption compressor cell . . . . . . . . . . . . . . 98

6.2 .2 Heat $\operatorname{sink} \ldots \ldots \ldots . \ldots \ldots 0$

6.2 .3 Check valves . . . . . . . . . . . . . . . . . . . . . . . . . . . . . . . . .

6.2 .4 Buffers ........................... 104

6.2.5 Heat exchangers . . . . . . . . . . . . . . . 104

6.3 Compressor assembling . . . . . . . . . . . . . . . 107

6.3 .1 Cell assembling . . . . . . . . . . . . . . 107

6.3 .2 Set-up assembling . . . . . . . . . . . . . . 109

6.4 Experiment and discussions . . . . . . . . . . . . . . . . 111

6.4.1 Preparation before the cool-down . . . . . . . . . . . 111

6.4 .2 Introductory test experiments . . . . . . . . . . . . 111

6.4.3 Single-cell experimental results . . . . . . . . . . . . . 114

6.4.4 Comparison of single-cell experiments with simulations . . . . 118

6.4 .5 Performance sensitivity . . . . . . . . . . . . . . 120

6.4.6 Multi-cells operation . . . . . . . . . . . . . . . 124

6.5 Conclusions ............................ 124

7 Scaled 40 K Neon-based Sorption Joule-Thomson Cooler 127

7.1 Overview of the scaled $40 \mathrm{~K}$ neon-based sorption JT cooler . . . . . . . 128

$7.240 \mathrm{~K}$ neon cold stage design and primarily testing . . . . . . . . . . . . 129

7.2 .1 CFHX ....................... 129

7.2 .2 Joule-Thomson restriction . . . . . . . . . . . . . . . . . . . . . . . . . . . . . . . . . . . . . . . . . . . . . .

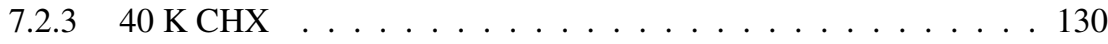

7.2 .4 Cold stage assembly . . . . . . . . . . . . . . . . 130

7.2.5 Experimental verification . . . . . . . . . . . . . 131

7.3 Neon sorption compressor design . . . . . . . . . . . . . . 136

7.4 Conclusions . . . . . . . . . . . . . . . . . . . 138

8 Conclusions and Outlook 139

8.1 Conclusions ........................... 140

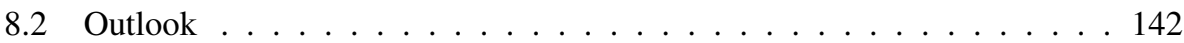

A Convection Term Approximation in 1-D Sorption Compressor Model 145 
Bibliography

149

Summary

Samenvatting (Summary in Dutch

161

Acknowledgements

165 
The universe is vast and full of wonders. It has persistently fascinated the curiosity of mankind since the prehistoric era. For thousands of years, the observation of the sky had been limited to the relatively narrow visible spectrum. Thanks to the modern electrooptical technology, humans perception has been widely expanded in both directions on the spectrum. Nowadays, we are able to record much more information from the universe than our ancestors did. To get high-quality imaging, the background thermal noise must be minimized. Therefore, cryogenic cooling is usually required in an astronomy imaging system to promise an acceptable resolution.

In the very first chapter of this thesis, some background information about the European Extremely Large Telescope (E-ELT) and the Mid-infrared E-ELT Imager and Spectrograph (METIS) is given, followed by a general introduction to the sorption cooling technology. The outline of the entire thesis is listed at the end of this chapter. 


\subsection{E-ELT \& METIS instrument}

Featuring an aperture of more than 20 m diameter, extremely large telescopes (ELT) are considered worldwide as one of the highest priorities in ground-based astronomy. However, ELTs are no new idea: 25-m class telescopes date back to the mid-70s [1]. Although these studies concluded that such telescopes were already technically feasible, the science case was not as strong as today permitted by adaptive optics, and underlying technologies were far less cost-effective than they are now. A few ELT concepts were proposed in the following decades [1], and successfully pioneered in the early 90s with the twin 10-m diameter Keck telescopes located on the top of Mauna Kea, Hawaii.

Since 1998, the European Southern Observatory (ESO) has been pursuing a conceptual study for a giant optical/infrared telescope with a primary mirror diameter up to 100 $\mathrm{m}$, dubbed for the eponymous bird keen night vision and for being Over Whelmingly Large [1-3]. ESO has worked in close cooperation with European industries on this original concept, with the goal of breaking the steep cost-to-diameter relation of the classical telescope approach.

Since the end of 2005, ESO has been working together with its user community of European astronomers and astrophysicists, to define the new giant telescope needed by the middle of the next decade. Following extensive community consultation through five working groups, the European Extremely Large Telescope (E-ELT), a revolutionary new concept of a ground-based telescope for optical/near-infrared range, was produced [4]. Figure 1.1 shows an artists impression of the E-ELT. This monster telescope will be built on a mountain top in Cerro Armazones, Chile, only $20 \mathrm{~km}$ from Cerro Paranal, home of ESOs Very Large Telescope (VLT) [5]. The telescope has an innovative five-mirror

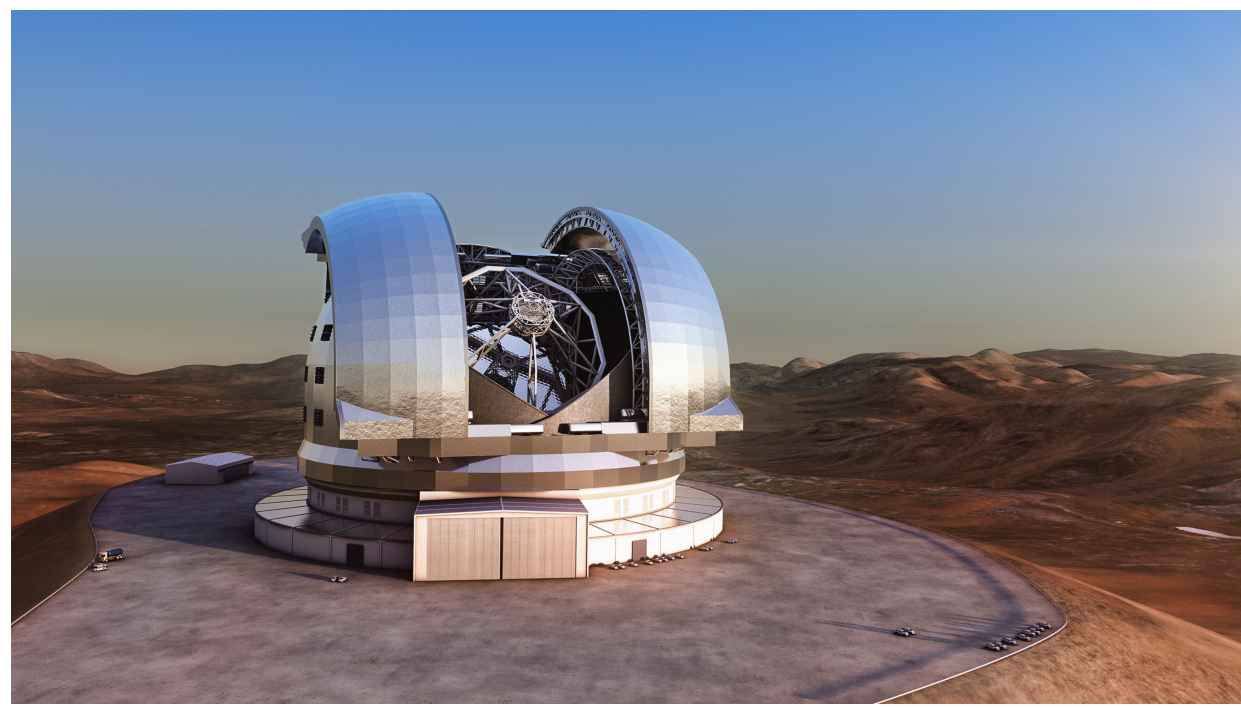

Figure 1.1. Artist impression of the E-ELT. 
design, and will be supported by adaptive optics and multiple instruments. Composed of 798 hexagonal segments, each $1.45 \mathrm{~m}$ wide, but only $50 \mathrm{~mm}$ thick, the primary mirror of E-ELT will be $39.3 \mathrm{~m}$ in diameter. With its unprecedented large eye on the sky, E-ELT will gather 15 times more light than the largest optical telescopes operating at the time of its development and vastly advance astrophysical knowledge and allow for detailed observations of among others the first objects in the universe and planets in other star systems [6].

E-ELT will have several scientific instruments, and it will be possible to switch from one instrument to another within minutes. Eight different instrument concepts and two post-focal adaptive modules are currently being studied. METIS, the Mid-infrared E-ELT Imager and Spectrograph, is one of those proposed instruments, and will offer imaging and spectroscopy over the wavelength range of 3-14 $\mu \mathrm{m}$, covering the $\mathrm{L}, \mathrm{M}$ and $\mathrm{N}$ bands $[7,8]$.

METIS consists of a warm part including instrumentation, structural supports and a vacuum vessel, and a cold part inside the vacuum vessel consisting of the cold optics and detectors [9] schematically depicted in Figure 1.2. The operating temperature levels of the imaging, dispersing and detecting subsystems of the instrument are determined by METIS radiometric performance. The detectors require approximately $40 \mathrm{~K}$ and $8 \mathrm{~K}$ for the $\mathrm{L} / \mathrm{M}$ and $\mathrm{N}$ band, respectively [7]. The temperatures of the optics, optomechan-

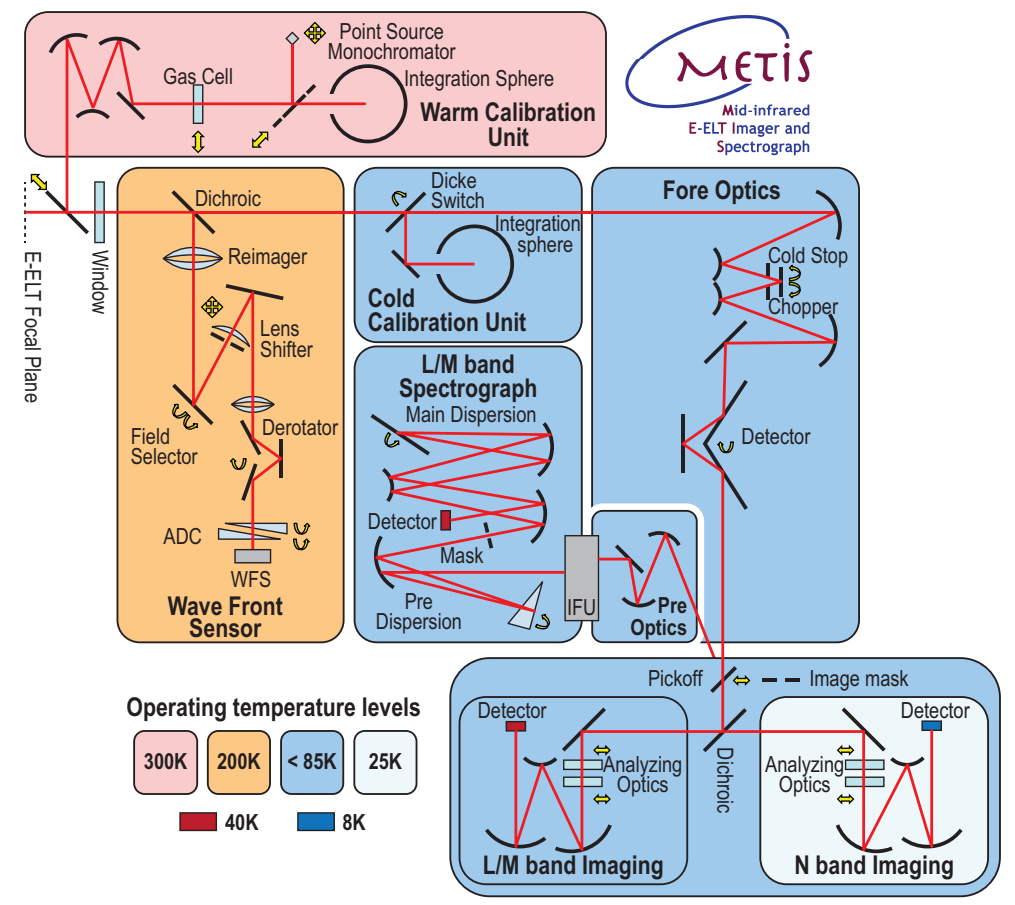

Figure 1.2. Optical overview of METIS instrument. 
Table 1.1. Required temperature levels and heat loads of METIS cryogenic units.

\begin{tabular}{lcc}
\hline METIS units & Required Max. temperature (K) & Heat load (W) \\
\hline N-Band Detectors & $\mathbf{8}$ & $\mathbf{0 . 4}$ \\
N-Band Imager & $\mathbf{2 5}$ & $\mathbf{1 . 1}$ \\
LM-Band Detectors & $\mathbf{4 0}$ & $\mathbf{1 . 4}$ \\
LM-Spectrometer & $<85$ & \\
LM-Imager & $<85$ & \\
Cold Calibration Unit & $<85$ & - \\
Cold Calibration Unit & $<85$ & \\
Fore Optics & $<85$ & \\
Radiation Shield & $<85$ & \\
\hline
\end{tabular}

ical components, and the thermal radiation shield are driven by their contribution to the overall noise budget that needs to be lower than the contributions from telescope and atmosphere. Performance analysis yields maximum allowable temperatures of $85 \mathrm{~K}$ for all cold modules, except for the N-band imager, which has to be cooled to below $30 \mathrm{~K}$ [7]. Furthermore, in configuration trade-offs, the number of different temperature levels has been reduced to four, being listed in Table 1.1.

A separate liquid nitrogen (LN2) bus system will cool the radiation shield and a backbone. All $85 \mathrm{~K}$ units will be thermally attached to the backbone. The three lower temperature levels, i.e. $40 \mathrm{~K}, 25 \mathrm{~K}$ and $8 \mathrm{~K}$, will be provided by cryocooler(s). The respective heat loads at these temperature levels are also shown in Table 1.1.

A key factor in the design of METIS is limiting the level of vibrations introduced at the detectors by the cooling system. Conventional cooling solutions such as Stirling or pulse-tube coolers have mechanically moving parts and require dedicated design measures with associated extra costs and risks to reduce the vibrations at the detector level. Failure to properly reduce vibrations can lead to a significant reduction in the optical performance of the instrument. Equally important is the short-term temperature stability of the cooling system at the cryogenic interfaces to prevent calibration errors due to changing detector temperatures. Furthermore, reliability is directly linked to the availability of the instrument. Sorption coolers, as discussed in more detail in the following sections, contain no moving parts, thus generate no vibrations, and have a long lifetime. Furthermore, a high temperature stability can be realized. Therefore, a vibration-free cooling technology based on sorption coolers is proposed for the METIS instrument.

\subsection{Sorption cooler \& its history}

Figure 1.3 shows an artist impression of a typical sorption Joule-Thomson (JT) cooler. It is composed of a sorption compressor and a JT cold stage. Apart from a few passive valves, sorption JT coolers have no moving parts, which is attractive for a number of 


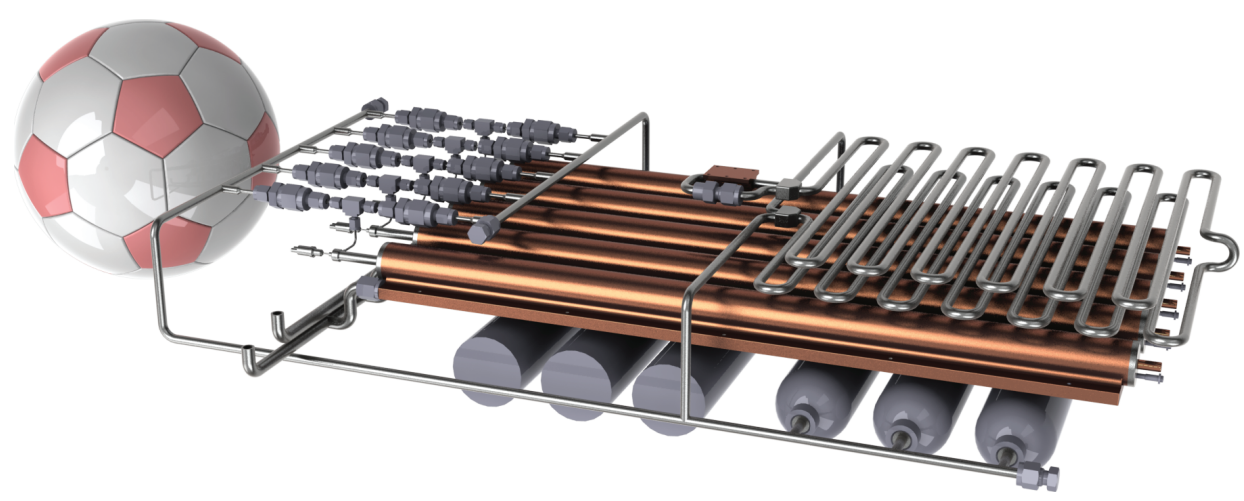

Figure 1.3. Artist impression of a $40 \mathrm{~K}$ neon sorption JT cooler.

reasons: they are vibration-free, EMI-free and have the potential of a long lifetime [10]. They also give flexibility in the integration of the instrument.

It was not until 1963 that sorption cooler concepts were proposed by Vickers of NASAs Jet Propulsion Laboratory (JPL) while the similar absorption refrigerators had been widely used for industrial and household applications for decades. Vickers suggested using silica gel as the adsorbent and a JT expander to provide cooling for long life spacecraft. The idea was eventually patented in 1966 [11].

However, no sorption cooler was actually built until the early 1970s, when van Mal and Mijnheer at the Philips Research Laboratories delivered approximately $1 \mathrm{~W}$ of cooling power at $26 \mathrm{~K}$ by using a LaNi5 chemical sorption compressor [12]. The development of a physical sorption cooler was first initiated by Hartwig of the University of Texas since 1975 . He suggested a variety of alternative physical sorption JT working fluid including $\mathrm{Ar}, \mathrm{N}_{2}, \mathrm{~N}_{2} \mathrm{O}, \mathrm{NH}_{3}$ and $\mathrm{He}$, and successfully demonstrated a physical sorption cooler achieving $3 \mathrm{~W}$ at $185 \mathrm{~K}$ incorporating zeolites and $\mathrm{N}_{2} \mathrm{O}$ [13-15].

Following these early efforts, several sorption cooler configurations were proposed and demonstrated [16-26], among which an important demonstration was the Brilliant Eyes Ten-Kelvin Sorption Cryocooler Experiment (BETSCE). BETSCE was designed to demonstrate $10 \mathrm{~K}$ sorption cooler technology in a space environment and cool infrared detectors to $10 \mathrm{~K}$ and below. It eventually was flown on Space Shuttle mission STS-77, and cooled down from $70 \mathrm{~K}$ to $10 \mathrm{~K}$ in less than 2 minutes and sustained a simulated I2R detector heat load of $100 \mathrm{~mW}$ for 10 minutes [27-30].

Another significant milestone of sorption cooler development is the $20 \mathrm{~K}$ Planck hydrogen cooler [31, 32], the first sorption cooler launched for a long term space mission. It was designed for the European Space Agency (ESA) Planck mission, to provide a total of $1 \mathrm{~W}$ cooling power for cooling the Planck Low Frequency Instrument (LFI) around $20 \mathrm{~K}$ while providing a pre-cooling for a $4 \mathrm{~K} \mathrm{JT}$ mechanical refrigerator for the High Frequency Instrument (HFI). Fabricated and assembled by the JPL, two coolers (one for redundancy), were finally launched with the Planck spacecraft on May 14th, 2009, served 
the instruments successfully for almost 4.5 years until the mission was completed on 23 October 2013 [33].

\subsection{Motivation \& research roadmap}

Sorption coolers have been developed for over a decade at the University of Twente in collaboration with Airbus Defence and Space Netherlands (formerly Dutch Space B.V.). In 1997, Burger et al. started with exploring the possibility of combining a micro JT cooler with a sorption compressor. Extensive investigations on sorption coolers were initiated, including adsorbent material characterization, thermodynamic analysis and modeling of sorption compressors, gas-gap heat switch (GGHS) and check valve design. These studies resulted in a first sorption cooler prototype at the University of Twente in 2001 $[34,35]$. Later, Wiegerinck started his Ph.D. research focusing on further improving the performance of sorption compressors for cryogenic cooling [36-38].

Funded by ESA Technological Research Programme (TRP), a 4.5 K helium sorption cooler for the ESA Darwin mission was developed, manufactured and tested $[39,40]$ at the University of Twente. It was able to produce $4.5 \mathrm{~mW}$ cooling power at $4.5 \mathrm{~K}$ with an input power of $1.96 \mathrm{~W}$ at a heat-sink temperature of $50 \mathrm{~K}$. The temperature stability in 1 hour is $1 \mathrm{mK}$ with controlling. Moreover, the cooler was operated continuously for 4 months with no performance degradation. Mechanical analysis predicts total exported vibration at a level less than $1 \mu \mathrm{N} / \mathrm{Hz}^{0.5}$ in the frequency band 0.1 to $1 \mathrm{~Hz}$.

Base on the success of the $4.5 \mathrm{~K}$ helium sorption cooler, the development continued on the hydrogen sorption cooler for the ESA Darwin mission [41]. This cooler reached a cold head temperature of $14.5 \mathrm{~K}$ with a net cooling power of $18.5 \mathrm{~mW}$ [10].

Enabled through the Netherlands Research School for Astronomy (NOVA) by financial support from the Netherlands Organization for Scientific Research (NWO) under contract 184.021.006, this Ph.D project focuses on developing a vibration-free cryogenic cooling solution based on sorption cooler for the METIS instrument in the E-ELT. This research is characterized by challenges such as multiple cooling levels, large cooling capacity within considerable efficiency and size, manufacturability and costs, etc.. The development that was carried out in this project following a roadmap:

Initialization: In this level, the basic principles related to the sorption cooler technology were gathered and studied, as well as the basic inputs (e.g. the fluid properties; suppliers, specifications, and characterizations of the adsorbent materials; and the cryogenic properties of the materials). Furthermore, the specifications and limitations of the cooler were defined, which is associated with Astron, the Netherlands Institute for Radio Astronomy.

Baseline design formulation: A first impression of the METIS cooler was presented at this level. A conceptual baseline design of the cooler chain for the METIS instrument was raised. Efforts were focused on the selection of the working fluid, the cooler chain layout 
and the preliminary evaluation of the cooler performance and size. The most critical components were labeled to be examinated in the next level.

Critical components design: Numerical models were built to study analytically the critical components in the METIS cooler, which is followed by optimizations and modifications. Detailed design of these components was carried out which instructed the experimental demonstrations in the following levels.

Submodule validation: Critical submodules including JT cold stage and sorption compressor unit were demonstrated to validate the analytical design and technical fabrication.

Full cooler stage validation: Associated with Airbus Defence \& Space Netherlands, a demonstrator at the cooler level is under development to present the feasibility of the sorption cooling technology for this particular large-scale ground application.

\subsection{Outline}

This thesis describes the research done to develop a vibration-free cryogenic cooling solution based on sorption cooler for the METIS instrument in the E-ELT. Following aspects will be detailed presented in this thesis:

- Chapter 1 gives a general introduction to the project and its background including sorption cooler development history, previous relevant work at the University of Twente, and a roadmap of this research.

- Chapter 2 starts with the basic thermodynamic cycles of a JT cold stage and a sorption compressor, followed by the background information about the adsorption phenomenon. As an essential input data for modeling a sorption compressor, the characterization of the adsorbent-adsorbate pair is discussed briefly. Finally, a general method for optimizing the working fluid for a sorption JT cooler is introduced.

- Chapter 3 presents a conceptual baseline design of the METIS cooler chain. It focuses on the multi-staging layout and the optimization of the operating parameters, resulting a preliminary estimation of the cooler chain performance and size.

- In Chapter 4, the detail design of the JT cold stages in the METIS cooler chain is presented. The multi-staging layouts for the cooler chain are compared, discussed and finalized. The required effectiveness of the counter flow heat exchangers (CFHXs) in the METIS cooler chain is defined as the target for the CFHX design. Then the detail design, fabrication, and measurement of a full-scale $8 \mathrm{~K}$ helium JT cold stage demonstrator are discussed.

- Chapter 5 describes the design of the sorption compressor for the METIS cooler chain. This design is based on a new concept that greatly increases the manufacturability and reduces the costs by canceling the GGHS. A dynamic model was developed to predict the performance of the sorption compressor. Furthermore basic dimensions 
and operating parameters for the sorption compressors in the METIS cooler chain were determined.

- Following the design in Chapter 5, a scaled helium sorption compressor demonstrator was design, manufactured and tested which is the major content of Chapter 6.

- Chapter 7 presents the demonstration of a scaled neon sorption cooler. The design of this cooler is described. The preliminary breadboard testing performed on the JT cold stage is discussed, and the current status of this cooler is stated.

- This thesis ends with conclusions and outlook in Chapter 8. 


\section{Fundamentals of Joule-Thomson Cold Stage and Sorption Compressor}

In this chapter, the basic thermodynamics related to a sorption Joule-Thomson (JT) cooler is introduced. It starts with the JT cold stage by describing the cooling cycle and the thermodynamic performance analysis. Then, the compression cycle in a sorption compressor is presented combined with some fundamentals of the adsorption phenomenon, including the comparison of physisorption and chemisorption and the thermal properties of the adsorbed fluid. Furthermore, a lumped, static analysis is performed on a sorption compressor to evaluate its performance. Finally, based on the thermodynamic analysis of the JT cold stage and the sorption compressor, a general method to optimize the working fluid for a sorption JT cooler is introduced. 


\subsection{Joule-Thomson cold stage}

\subsubsection{Basic Joule-Thomson cold stage: Linde-Hampson system}

A basic JT cold stage uses the Linde-Hampson cycle, as shown in Figure 2.1. It consists of a counter flow heat exchanger (CFHX), a JT restriction, and an evaporator. It is driven by a compressor at ambient temperature, $T_{a m b}$. Mechanical compressors usually operate at room temperature $(\sim 293 \mathrm{~K})$, whereas sorption compressors can run in a large temperature range from room temperature to cryogenic temperature. In a compressor, the working fluid is compressed adiabatically from low pressure to high pressure. The compressor is usually equipped with an aftercooler that, cools the compressor out-flow to ambient temperature (with a perfect aftercooler).

The warm pressurized fluid enters the CFHX and is cooled down (1-2) by the lowpressure fluid that flows in the opposite direction. The precooled high-pressure fluid then flows through the JT restriction and expands isenthalpically to low pressure (2-3). If the working fluid is precooled sufficiently that its temperature before the JT expansion is lower than the inversion temperature, the fluid then experiences a decrease in temperature via the expansion (down to the cooling temperature, $T_{c}$ ) and it is partly liquefied (point 3 ).

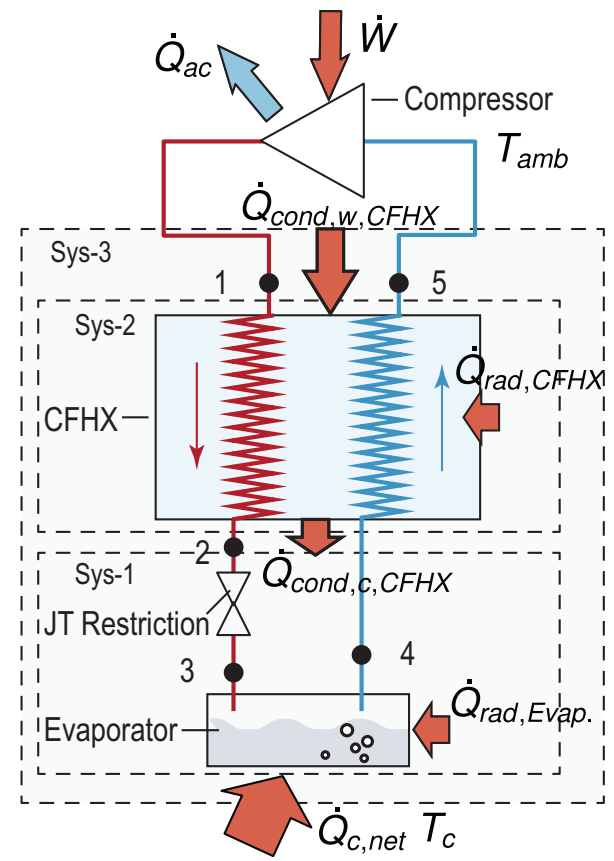

Figure 2.1. Schematic of a basic JT cold stage using the Linde-Hampson cycle. Driven by a compressor, a basic JT cold stage is composed of a counter flow heat exchanger (CFHX), a JT restriction, and an evaporator. 
The evaporator takes the heat load resulting from the device to be cooled and evaporates the liquid (3-4). The saturated low-pressure vapor (point 3) leaves the evaporator and flows back through the CFHX. The cold low-pressure fluid is heated up in the CFHX in its return to the compressor (4-5).

\subsubsection{Thermodynamic analysis for a basic Joule-Thomson cold stage}

For the thermodynamic analysis of the basic JT cold stage, it is assumed that,

1. the working fluid is a pure fluid (mixture JT cooling is not in the scope of this thesis);

2. the working fluid at the outlet of the compressor has a temperature of $T_{a m b}$ (perfect aftercooler) and a high pressure of $p_{h}$ (pre-defined, but to be optimized);

3. the working fluid expands isenthalpically through the JT restriction;

4. only saturated vapor returns to the CFHX;

5. the system operates at a steady state.

The cycle process is presented on a pressure-enthalpy $(\mathrm{p}-\mathrm{h})$ diagram as shown in Figure 2.2. The cooling temperature of a JT cold stage with a pure working fluid determines the pressure in the evaporator [42]. Combined with assumption 4 , the thermodynamic state of the flow at point 4 in Figure 2.2 is fully defined. The state at point 1 is also defined according to assumption 2.

Apply the first law of thermodynamics on the system that only consists of the CFHX, i.e. Sys-2 dashed-line box as shown in Figure 2.1,

$$
\underbrace{\dot{H}_{1}+\dot{H}_{1}+\dot{Q}_{\text {cond,w,CFHX }}+\dot{Q}_{\text {rad }, C F H X}}_{\text {Energy flow in }}=\underbrace{\dot{H}_{2}+\dot{H}_{5}+\dot{Q}_{\text {cond }, c, C F H X}}_{\text {Energy flow out }}
$$

Rearrange the equation,

$$
\underbrace{\left(\dot{H}_{1}-\dot{H}_{2}\right)}_{\dot{Q}_{h, t r, \text { CFHX }}}-\underbrace{\left(\dot{H}_{5}-\dot{H}_{4}\right)}_{\dot{Q}_{l, t r, \text { CFHX }}}+\underbrace{\left(\dot{Q}_{c o n d, w, C F H X}-\dot{Q}_{c o n d, c, C F H X}\right)}_{\Delta \dot{Q}_{\text {cond }, \text { CFHX }}}+\dot{Q}_{r a d, C F H X}=0
$$

where $\dot{Q}_{h, t r, C F H X}$ and $\dot{Q}_{l, t r, C F H X}$ are the heat flows in the CFHX from the high-pressure gas flow to the low-pressure gas flow, respectively. These are equal, i.e. the segment 1-2 has the same length as the segment 4-5 as shown in Figure 2.2, in an ideal situation in which there are no conduction and radiation contributions.

Again, apply the first law of thermodynamics on the system that consists of the JT restriction and the evaporator, i.e. Sys-1 dashed-line box as shown in Figure 2.1,

$$
\underbrace{\dot{H}_{2}+\dot{Q}_{\text {cond }, c, C F H X}+\dot{Q}_{\text {rad,Evap. }}+\dot{Q}_{c, \text { net }}}_{\text {Heat flow in }}=\underbrace{\dot{H}_{4}}_{\text {Heat flow out }}
$$


It is rearranged as,

$$
\dot{Q}_{c, n e t}=\underbrace{\left(\dot{H}_{4}-\dot{H}_{2}\right)}_{\dot{Q}_{c, \text { gross }}}-\dot{Q}_{c o n d, c, \text { CFHX }}-\dot{Q}_{\text {rad,Evap }} .
$$

Here, the gross cooling power of the cold stage is defined as the difference in enthalpy flows at points 4 and 2. Subtracting the conduction loss at the cold end of the CFHX and the radiation loss on the evaporator from this gross cooling power results in the net cooling power.

By combining Eq. 2.2 and Eq. 2.4, one can obtain the energy balance equation for the entire JT cold stage, i.e. Sys-3 desh-line box shown in Figure 2.1,

$$
\dot{Q}_{c, n e t}+\dot{Q}_{c o n d, w, C F H X}+\dot{Q}_{\text {rad }, \text { CFHX }}+\dot{Q}_{\text {rad }, \text { Evap. }}=\underbrace{\left(\dot{H}_{5}-\dot{H}_{1}\right)}_{\text {Cooling potential }}=\dot{m}\left(h_{5}-h_{1}\right)
$$

Here, a cooling potential is defined as the enthalpy difference between high- and lowpressure gas flows at the warm end of the CFHX.

In the primary stage of designing a JT cold stage, the ideal situation is usually considered where the conduction, radiation and pressure drop losses associated with the cold stage are neglected. Eq. 2.2 and Eq. 2.5 are reduced to,

$$
\begin{gathered}
\dot{Q}_{t r, C F H X}=\dot{H}_{1}-\dot{H}_{2}=\dot{H}_{5}-\dot{H}_{4} \\
\dot{Q}_{c}=\dot{H}_{5}-\dot{H}_{1}=\dot{m}\left(h_{5}-h_{1}\right)
\end{gathered}
$$

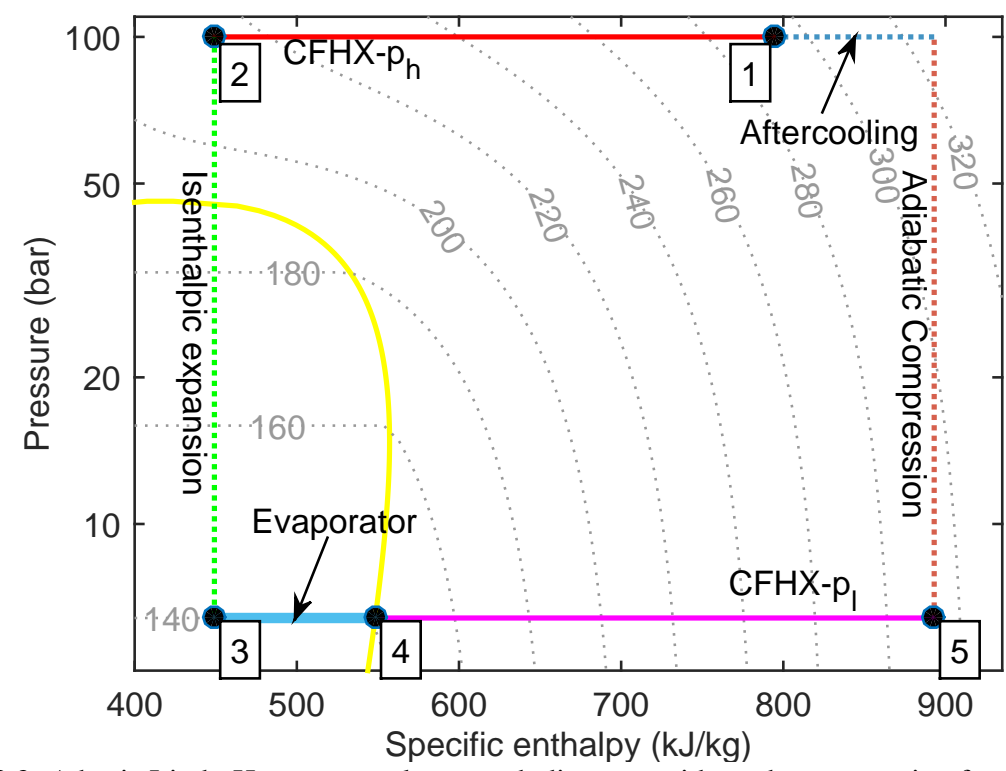

Figure 2.2. A basic Linde-Hampson cycle on a p-h diagram, with methane operating from $293 \mathrm{~K}$ to $140 \mathrm{~K}$ with a high pressure of $100 \mathrm{bar}$. The gray dashed lines are the isotherms and the numbers on isotherms indicate the temperatures in Kelvin. The yellow thick line is the saturation dome. 
By introducing the effectiveness E of the CFHX, the heat transferred in the CFHX can be written as,

$$
\dot{Q}_{t r, C F H X}=E \dot{Q}_{t r, C F H X, \max }
$$

Note that the effectiveness of the CFHX is defined as the ratio of the heat that is actually transferred from warm flow to cold flow to the maximum heat that can possibly be transferred between the two flows [43]. The maximum heat that can possibly be transferred in a CFHX equals,

$$
\dot{Q}_{t r, \max }=\dot{m} \min (\underbrace{h\left(T_{1}, p_{l}\right)-h_{4}}_{\text {when } T_{5}=T_{1}}, \underbrace{h_{1}-h\left(T_{4}, p_{h}\right)}_{\text {when } T_{2}=T_{4}})
$$

Therefore, the required flow rate for a given cooling power of the JT cold stage can be evaluated by combining Eq. 2.6-2.9,

$$
\dot{m}=\frac{\dot{Q}_{c}}{\left(h_{4}-h_{1}\right)+E \min \left(h\left(T_{1}, p_{l}\right)-h_{4}, h_{1}-h\left(T_{4}, p_{h}\right)\right)}
$$

Furthermore, the coefficient of the performance (COP) of a JT cold stage is defined by the ratio of the cooling power to the minimum compression power,

$$
C O P_{J T C S}=\frac{\dot{Q}_{c}}{\dot{W}_{c o m p r, \text { min }}}=\frac{\dot{Q}_{c}}{\dot{m} \Delta g_{\text {compr }}}=\frac{\dot{Q}_{c}}{\dot{m}\left[\left(h_{1}-T_{1} s_{1}\right)-\left(h_{5}-T_{5} s_{5}\right)\right]}
$$

Eq. 2.10 and Eq. 2.11 are useful for optimizing several critical parameters in the JT cold stage, such as the high pressure and the required effectiveness of the CFHX.

In the further design of the components in the JT cold stage, losses due to the nonideality, such as longitude conductions, radiations, and pressure drops, shall be considered and calculated in details. Nevertheless, loss terms will usually degrade the performance of the JT cold stage. It is wise to consider reasonable margins during the preliminary design.

\subsubsection{Precooled Joule-Thomson cold stage}

Reducing the inlet temperature of the CFHX is, in general, attractive since it increases the enthalpy difference at the warm end of the CFHX and thus the performance of the cold stage. In order to obtain temperatures below $40 \mathrm{~K}$ precooling to well below $300 \mathrm{~K}$ is even required since the candidate working fluids (neon, hydrogen, and helium) have their so-called inversion temperatures below $300 \mathrm{~K}$ [44].

A JT cold stage with a pre-cooling stage is illustrated schematically in Figure 2.3 and its cycle process on the p-h diagram in Figure 2.4. To analyze such a system, the assumptions described in Sec. 2.1.1 remain. Additionally, a few assumptions are made as follows,

6. the pre-cooling is only applied to the high-pressure line, 


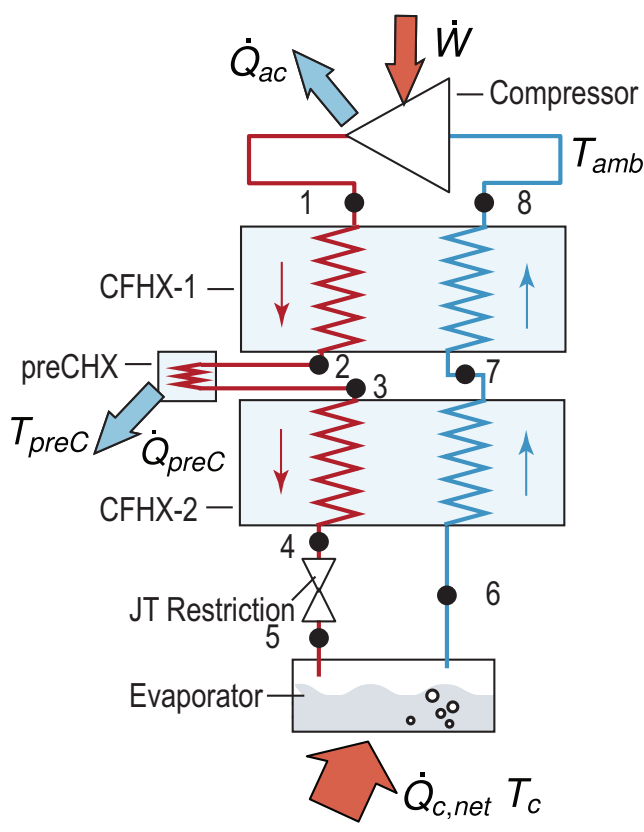

Figure 2.3. Schematic of a JT cold stage with pre-cooling stages. preCHX: precooling heat exchanger.

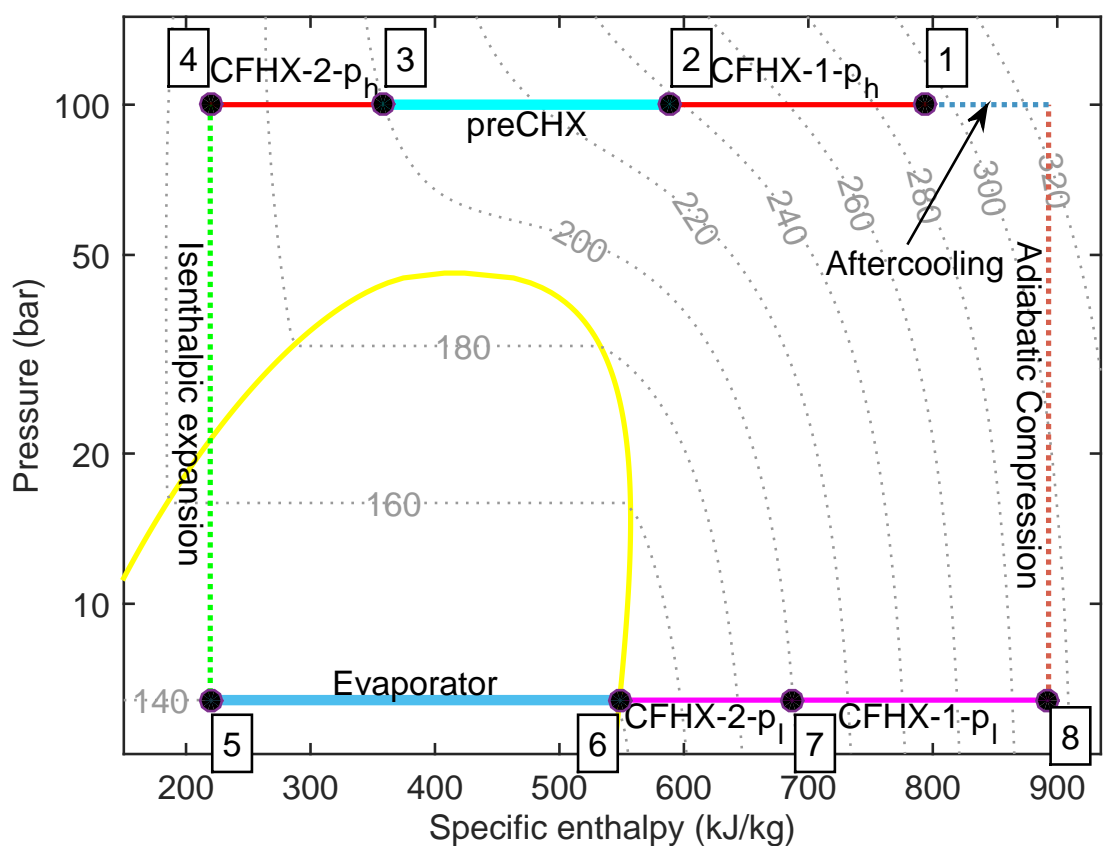

Figure 2.4. A pre-cooled Linde-Hampson cycle on a p-h diagram, with methane operating from $293 \mathrm{~K}$ to $140 \mathrm{~K}$, pre-cooling of $200 \mathrm{~K}$ and a high pressure of $100 \mathrm{bar}$. The gray dashed lines are the isotherms and the numbers on isotherms indicate the temperatures in Kelvin. The yellow thick line is the saturation dome. 
7. the pre-cooling source is considered as a cold heat-sink with a constant pre-cooling temperature, $T_{\text {preC }}$,

8. the pre-cooling heat exchanger (preCHX) is perfect. Therefore, the outflow temperature of the preCHX equals the precooling temperature.

With these assumptions, the thermodynamic statuses of the flow at point 1,3 , and 6 are fully defined. The analysis starts from the last CFHX, made in analogy with a basic JT cold stage operating at an ambient temperature that equals $T_{\text {prec }}$. Considering an ideal situation and replacing the subscripts in Eq. 2.10, one can find the required flow rate is only dependent on the effectiveness of the last CFHX and the required cooling power,

$$
\dot{m}=\frac{\dot{Q}_{c}}{\left(h_{6}-h_{3}\right)+E_{2} \min \left(h\left(T_{3}, p_{l}\right)-h_{6}, h_{3}-h\left(T_{6}, p_{h}\right)\right)}
$$

The flow rate is directly related to the performance of the JT cold stage of interest since it is proportional to the compression work needed in the compressor. Therefore the last CFHX is, in most cases, the most critical CFHX in a pre-cooled JT cold stage. However, the rest of CFHX will influence the per-cooling power that is related to the performance of the entire cooling system. And the required pre-cooling power at $T_{\text {preC }}$ can be expressed by,

$$
\dot{Q}_{\text {preC }}=\dot{m}\left[h_{1}-h_{3}-E_{1} \min \left(h\left(T_{1}, p_{l}\right)-h_{7}, h_{1}-h\left(T_{7}, p_{h}\right)\right)\right]
$$

JT cold stage with multiple pre-cooling stages can be studied in the similar way. Comparing Figure 2.2 and Figure 2.4, one can find that by applying pre-cooling the enthalpy difference available at the evaporator increases significantly, resulting a reduce in the required flow rate pressure drop through the CFHX. Consequently, the COP of the JT cold stage is essentially improved. The pre-cooling is usually preferable if there is an (or several) existing cold heat-sink source that can provide the pre-cooling relatively efficiently.

\subsection{Sorption compressor}

\subsubsection{Compression cycle in a sorption compressor}

A sorption compressor is based on the principle that a large amount of gas can be adsorbed physically or absorbed chemically by certain solids such as activated carbon, metal-organic framework (MOF), praseodymium-cerium-oxide (PCO) or metal hydride [45]. A single-stage sorption compressor as considered in this thesis is shown in Figure 2.5 [37]. Since the sorption cell adsorbs and desorbs the working fluid in a cyclic manner, passive check valves are required to maintain the correct flow direction through the JT cold stage. Furthermore, buffer volumes are applied to stabilize the pressure difference over the cold stage. Via thermal switches, the sorption cell is connected to a heat sink that can be a convectively cooled unit for ground applications or a radiator in space. In order to 


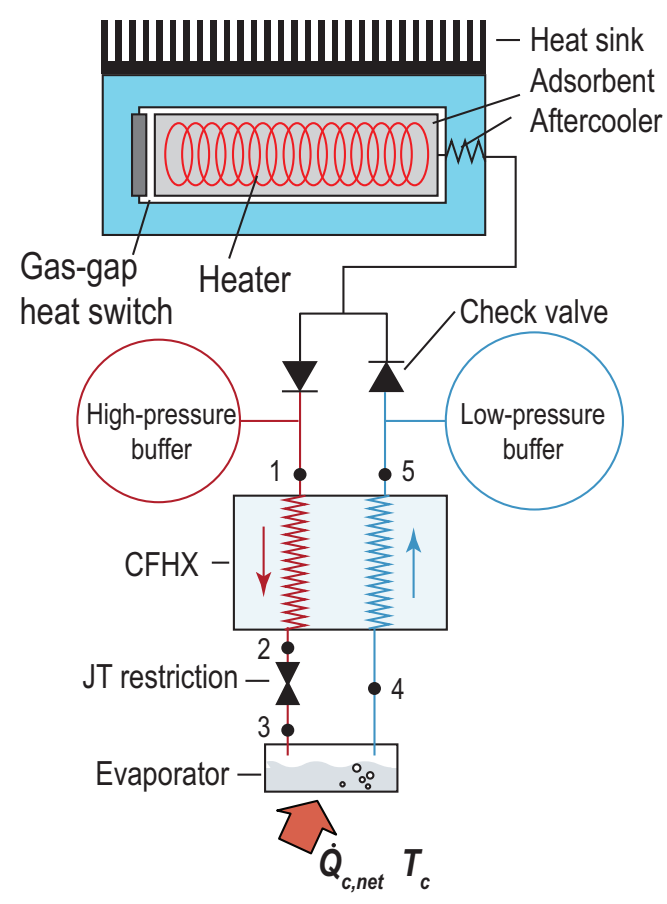

Figure 2.5. Schematic of a single-stage sorption compressor driving a JT cold stage for cryogenic cooling.

generate higher flow rates, or for redundancy reasons, multiple cells are usually arranged in parallel.

The cycle of a single-stage sorption compressor, as shown in an adsorption-isotherm diagram in Figure 2.6, can be divided into four phases that are compression, out-flow, decompression and in-flow.

Starting from point $\mathrm{A}$ at initial low temperature $T_{l}$ and low pressure $p_{l}$, the sorption cell is heated while thermally isolated from the heat sink. In this compression phase (a-b in Figure 2.6) both check valves are closed. As the temperature increases, the adsorbed gas is released and fills the void volume in the cell. As a result, the pressure in the cell container increases. In this phase, the adsorbed amount of gas per unit of mass of adsorbent, $x_{a d s}$, decreases but the total amount of the gas in the cell remains the same.

When the pressure in the cell excesses the high pressure $p_{h}$ (point $\mathrm{b}$ in Figure 2.6), the check valve on the high-pressure side opens, and the pressurized gas flows out of the cell while the heating continues. In this out-flow phase (b-c in Figure 2.6), the pressure in the cell is maintained at $p_{h}$ as the buffer volumes are large enough to stabilize the pressure.

When most of the adsorbed gas is desorbed and the cell has been heated up to a high temperature of $T_{h}$ (point $\mathrm{c}$ in Figure 2.6), heating is stopped and the cell is cooled down. The high-pressure check valve closes at that moment, and the pressure in the cell starts to drop. In this decompression phase (c-d in Figure 2.6), gas is adsorbed due to the temperature decrease in the sorption cell, and the pressure in the cell container consequently drops down until the initial low pressure $p_{l}$ is reached. 


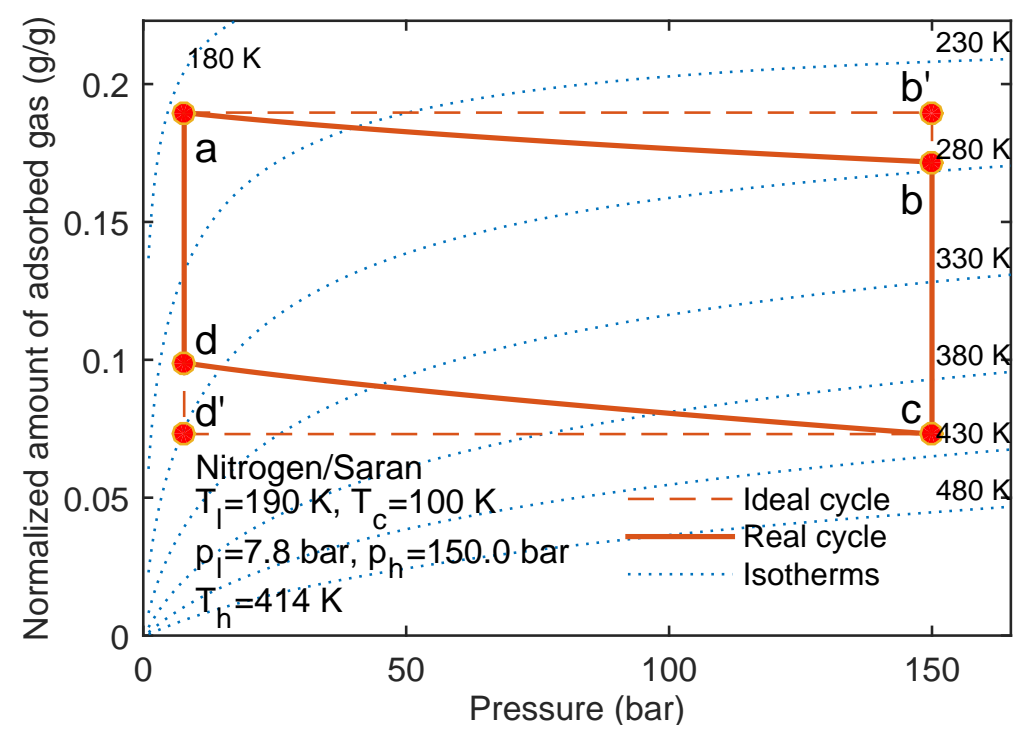

Figure 2.6. Example of a single-stage sorption compressor cycle operating with nitrogen and saran carbon. The blue dotted lines are the adsorption isotherms of nitrogen on saran carbon. a-b-c-d shows a cycle in an ideal adsorbent. Compression phase and decompression phase will deviate from the ideal process, as depicted by process a-b-c-d, because of the void volume in the adsorbent holding some amount of the working fluid during the cycle.

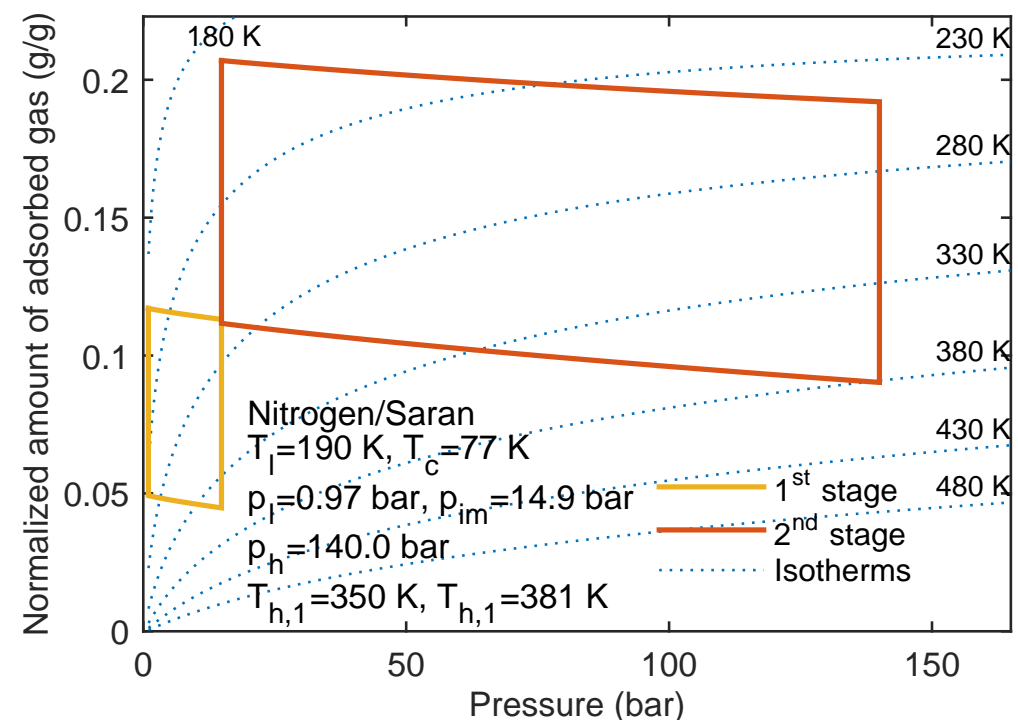

Figure 2.7. Example of a two-stage sorption compressor cycle running with nitrogen and saran carbon. 
The low-pressure check valve opens to start the in-flow phase (d-a in Figure 2.6). The low-pressure gas from the low-pressure buffer flows into the cell and is adsorbed onto the adsorbent material while the temperature further decreases until it reaching the startingpoint temperature $T_{l}$ which finishes a full compression cycle.

In a sorption compressor the void volumes within the adsorbent and in the connecting lines and check valves can significantly reduce the gas amount pumped by the sorption compressor in each cycle. These void volumes, therefore, decrease the cooling capacity of the system and raise its input power requirement. Obviously, the void volumes can be minimized by selecting a better adsorbent and by optimizing the compressor-cell design. The other method to reduce the void-volume effect, proposed by Bard [46], is to use multistage compression. A multistage compressor is basically composed of multiple singlestage compressor in serial to achieve higher pressure ratio and better efficiency. Figure 2.7 shows a compression cycle of a two-stage compressor. The gas is compressed to an intermediate pressure $p_{\text {im }}$ by the first stage and cooled down to its initial low temperature $T_{l}$ by flowing through an inter-aftercooler before entering into the second stage. The smaller pressure swing in each stage can significantly reduce the void-volume effect and increase the gas flow rate produced by the compressor, therefore improving the system performance.

\subsubsection{Fundamentals of the adsorption phenomenon}

Adsorption is the process by which the fluid molecules are collected on the solid surface causing an increase in the density of the fluid. The adsorption phenomenon is known for thousands of years. Adsorption of gasses on materials such as clay, sand and wood charcoal were utilized by the ancient Egyptians, Greeks and Romans [47]. Adsorption is of great importance in modern industry, used for instance in the separation of gasses, the purification of liquids and pollution control. Some adsorbent materials are also used as catalysts or catalyst supports whereas others play a vital role in the solid state reactions and biological mechanisms [48]. In high vacuum applications, the adsorbent materials are used for gas getting. Also, the adsorption phenomenon is utilized for thermal compression in the HVAC industry and cryogenic engineering.

\section{Physisorption versus Chemisorption}

The adsorption is caused by the interactions between the solid and the fluid molecules and is generally classified as physisorption or chemisorption depending on the kind of interaction force involved. Physisorption is mainly coupled to the van de Waals force that is also responsible for the vapor condensation, whereas chemisorption involves a chemical reaction between the solid surface and the fluid and forms stronger covalent and ionic bonds. In Table 2.1, the differences between physisorption and chemisorption are summarized [34, 48].

As indicated in Table 2.1, physisorption has a number of significant advantages compared to chemisorption, e.g. reversibility and generally applicable (not gas-specific). 
Table 2.1. Summary of differences between physisorption and chemisorption.

\begin{tabular}{ll}
\hline \multicolumn{1}{c}{ Physisorption } & \multicolumn{1}{c}{ Chemisorption } \\
\hline & \multicolumn{1}{c}{ Degree of specificity } \\
Low (Physisorption is a general phenomenon & High (Chemisorption is dependent on the re- \\
that happens at any interface between solid & activity of two substances) \\
and fluid) & \\
\hline
\end{tabular}

\section{Heat of adsorption}

Small, always exothermic (Same order as the Large (Same order as the energy change in a heat of liquefaction of the fluid) comparable chemical reaction, usually much larger than the heat of liquefaction)

\section{Adsorption degradation}

None, reversible process

Yes, irreversible process [45]

\section{Operating temperature}

Depending on the working fluid, large temperature range from room temperature to cryogenic temperature. However adsorption amount is limited at relatively high temperature.
Occurs above the certain temperature where the system has a thermal energy higher than the activation energy to attain the chemical reaction.

\section{Typical adsorbents}

Silica gel, activated carbon, zeolite, metal- Metal-hydride $\left(\mathrm{H}_{2}\right)$, praseodymium cerium organic framework oxide $\left(\mathrm{O}_{2}\right)$

Therefore, in this thesis the discussion is limited to physisorption.

\section{Isotherms data}

The sorption behavior of an adsorbent-adsorbate combination is a critical input to the sorption compressor model. This behavior can be measured by using a gravimetric or volumetric method [49] and represented by a series of isotherms in a plot of the amount of specific adsorbed gas as a function of the pressure, as shown by the thin dotted lines in Figure 2.6 and Figure 2.7. A large number of experimental hydrogen isotherms on various adsorbents, such as activated carbon, carbon nanotubes and graphite measured for storage purposes have been reported [50-52]. Isotherm data of other working fluids such as nitrogen, neon, and krypton, can also be found in literatures [53-55]. These literature data are certainly useful in a preliminary design phase. However, for detailed design, it is recommended to measure the isotherms of the particular working fluids and adsorbents. A number of theories have been developed for correlating isotherms to experimental data so as to predict the adsorption amount at a wide range of temperatures and pressures. Most of these theories are based on the potential theory of adsorption, which was originally proposed and experimentally substantiated by Polanyi $[56,57]$.

In the University of Twente, a sophisticated adsorption-isotherm measuring setup was 
built based on gravimetric method. A large number of isotherm measurements was carried out to produce input data for the sorption compressor development. In this thesis, helium, hydrogen and neon isotherms on saran carbon are of interest. The former two had been measured early years for the previous projects. The neon isotherm used in this study was provided internally by Jet Propulsion Laboratory, which is further verified by measuring several critical points on the isotherms.

\section{Thermal properties of the adsorbed fluid}

In order to evaluate the performance of a sorption compressor, it is necessary to know some basic thermal properties of the working fluid at the adsorbed phase, such as density and specific heat capacity. The adsorption mechanisms for the subcritical region and supercritical region are different. For the subcritical adsorption $\left(T<T_{c r}\right)$, the equilibrium pressure will not excess the saturation pressure, $p_{\text {sat }}$, or the non-adsorbed phase condenses. Therefore, it is reasonable to assume that the adsorbed phase of the adsorbate is in the form of a condensed liquid which is in a highly compressed state [57]. However, condensation will not happen regardless of the pressure in the supercritical region $\left(T>T_{c r}+10\right)$ [49]. The sorption compressor of interest to this thesis for JT cryogenic cooler usually operates at a heat-sink temperature significantly higher than the critical temperature. Thus the adsorbed phase is regarded as a sort of superheated, incompressible liquid and its density is only temperature dependent and given by a formula proposed by Ozawa [58],

$$
\rho_{a d s}(T)=\frac{\rho_{n b, l}}{\exp \left[\Omega\left(T-T_{n b}\right)\right]}
$$

where $\rho_{n b, l}$ and $T_{n b}$ are the liquid density and the temperature at the normal boiling point, and $\Omega$ indicates the thermal expansion of the superheated liquid which can be evaluated by $[59,60]$,

$$
\Omega=\frac{\ln \left(b \rho_{n b, l} / M\right)}{T_{c r}-T_{n b}}
$$

where $b$ is the van de Waals volume and $M$ is the molar mass.

For long time, the specific heat capacity of the adsorbed phase $c_{a d s}$ has been assumed to be equal to that of a condensed fluid at an equivalent state [61, 62]. However, in this thesis, $c_{a d s}$ is calculated by an equation proposed by Walton [63].

\subsubsection{Thermodynamic analysis of a sorption compressor}

In this section, a static analysis for a sorption compressor is performed based on the assumptions as follows,

1. there is no additional void volume in the sorption cell except the volumes of the pores in the adsorbent,

2. the pressure drop through the adsorbent pores is neglected, so that the pressure in the sorption cell is uniform during the cycle, 
3. adsorption and desorption are assumed to occur instantaneously, i.e. the adsorbed fraction directly follows temperature and pressure according to the isotherms,

4. the sorption cell is assumed as a lump, thereby its interior temperature essentially uniform during the thermal cycling,

5. the aftercooler is perfect, i.e. the working fluid exits the compressor-cell assembly at heat-sink temperature,

6. check valves are perfect, i.e. zero pressure drop and zero cracking pressure for the check valves,

7. the low temperature of the sorption cycle is equal to the heat-sink temperature $T_{H S}$,

8. stationary operation of the compressor is assumed.

The goal of this analysis is to find an expression for the coefficient of performance of a sorption compressor $C O P_{S C}$. The COP of a single stage sorption compressor $C O P_{S C, s s}$ is defined by the ratio of the input heat to the change in Gibbs free energy of the working fluid, written as $[36,38]$,

$$
C O P_{S C, s s}=\frac{\Delta \tilde{G}}{\tilde{Q}_{\text {input }}}=\frac{\Delta g \tilde{m}}{\tilde{Q}_{\text {input }}}=\frac{\Delta g \tilde{x}}{\tilde{q}_{\text {input }}}
$$

with $\Delta g$ the specific Gibbs free energy difference between the inflow and the outflow, $\tilde{m}$ the mass of working fluid cycled by the compressor per cycle, $\tilde{x}=\tilde{m} / m_{s}$ the mass of working fluid per unit of adsorbent material that is pumped by the compressor per cycle, and $\tilde{q}_{\text {input }}=\tilde{Q}_{\text {input }} / m_{s}$ is the input heat per cycle per unit of adsorbent material.

Since the aftercooler is assumed to be perfect, the outflow of the compressor is precooled to the heat-sink temperature $T_{H S}$ before it enters the cold stage while the inflow of the compressor is heated from a temperature of $T_{i n}$ to $T_{H S}$ before it enters the sorption cell. Then $\Delta g$ can be expressed as $[34,64]$,

$$
\Delta g=g_{\text {out }}-g_{\text {in }}=\left[h\left(T_{H S}, p_{h}\right)-T_{H S} s\left(T_{H S}, p_{h}\right)\right]-\left[h\left(T_{\text {in }}, p_{l}\right)-T_{\text {in }} s\left(T_{\text {in }}, p_{l}\right)\right]
$$

where $s(T, p)$ denotes the specific entropy of the working fluid as a function of temperature and pressure. Note that $T_{i n}$ is the temperature of the gas flowing into the compressorcell assembly. It usually equals to the exit temperature of the low-pressure line in the CFHX.

$\tilde{x}$ can be expressed as the total swing in amount of the fluid in the sorption cell normalized to the mass of the adsorbent material, written as,

$$
\tilde{x}=x_{t o t, a}-x_{t o t, c}=\left(x_{a d s}+x_{f r e}\right)_{a}-\left(x_{a d s}+x_{f r e}\right)_{c}
$$

where $x_{a d s}$ is the normalized amount of adsorbed fluid that is attached to the surface of the adsorbent material forming a thin layer of condensed phase, whereas $x_{f r e}$ is the normalized amount of free fluid that is not adsorbed but occupies the void volume inside the adsorbent material. The subscripts "a" and "c" represent the states "a" and "c" illustrated in Figure 2.6. The normalized amount of adsorbed fluid is measured experimentally, 
and usually presented through isotherms, as the blue dotted lines illustrated in Figure 2.6 and Figure 2.7. The normalized amount of free fluid in the sorption cell as a function of temperature and pressure results as $[36,38]$,

$$
x_{\text {fre }}(T, p)=\rho_{\text {fre }}(T, p)\left(\frac{\alpha}{\rho_{\text {sor }, t}(1-\alpha)}-\frac{x_{\text {ads }}(T, p)}{\rho_{\text {ads }}(T, p)}\right)
$$

Here $\rho_{f r e}$ is the density of the non-adsorbed free fluid, $\rho_{s o r, t}$ is the true density of the adsorbent material, $\rho_{a d s}$ is the density of the adsorbed fluid, and $\alpha$ is the porosity of the adsorbent material.

To investigate the required normalized input heat per cycle in Eq. 2.16, the energy balance of the cell during a compression cycle has to be studied. The adsorption part in the cell can be considered as a three-component mixture that consists of the porous solid adsorbent, the adsorbed fluid and the free fluid.

By assuming the free fluid as an ideal gas, the enthalpy change of such a mixture in the compressor cell is composed of (a.1) the sensible heat of each component caused by the temperature change and (a.2) the heat of adsorption (one can consider it as the latent heat of the adsorption phenomenon), as shown in Figure 2.8. The energy flow coming into and out of the compressor cell includes (b) the input heating or cooling, and (c) the

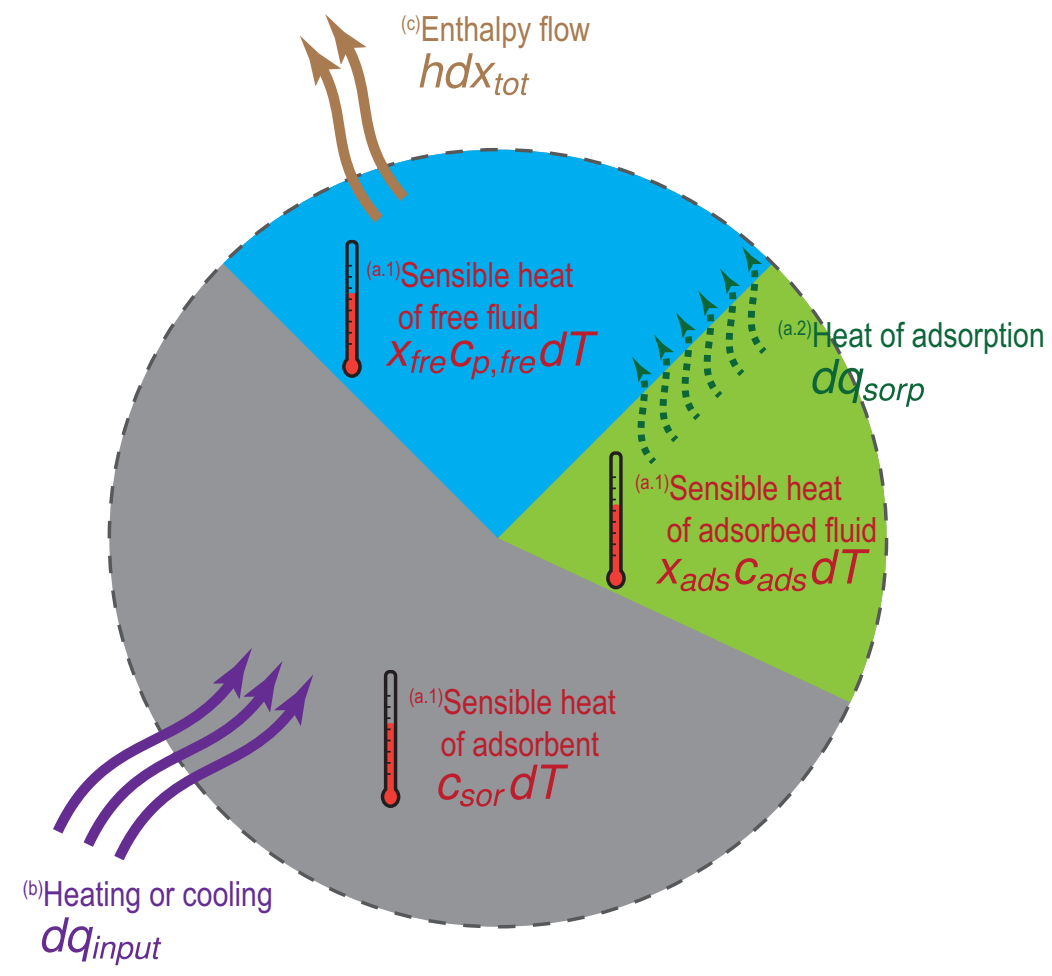

Figure 2.8. Energy balance of the compressor cell. 
enthalpy flow corresponding to the mass flow in or out. Therefore, the normalized energy balance can be written as,

$$
\underbrace{\underbrace{\left(c_{\text {sor }}+x_{\text {ads }} c_{\text {ads }}+x_{\text {fre }} c_{p, f r e}\right) \mathrm{d} T}_{\text {(a.2) heat of adsorption }}}_{\text {(a.1) sensible heat }}+\underbrace{\mathrm{d} q_{\text {sorp }}}_{\begin{array}{c}
\text { (b) heating } \\
\text { or cooling }
\end{array}}+\underbrace{h \mathrm{~d} x_{\text {tot }}}_{\begin{array}{c}
\text { (c) enthalpy } \\
\text { flow }
\end{array}}
$$

(a) enthalpy change

The heat of adsorption representing the enthalpy change of the system due to the redistribution of the working fluid between the adsorbed phase and non-adsorbed phase from one adsorption equilibrium to another, is derived from the isotherms, given by [48],

$$
\mathrm{d} q_{\text {sorp }}(T, p)=\left.\frac{R}{M} \frac{\partial \ln (p)}{\partial(1 / T)}\right|_{x_{a d s}} \mathrm{~d} x_{a d s}=-\left.\frac{R T^{2}}{M p} \frac{\partial p}{\partial T}\right|_{x_{a d s}} \mathrm{~d} x_{a d s}
$$

Furthermore, there are thermal masses other than the adsorption part, such as the cell container, which is also thermally cycled between the high and low temperatures. By adding these contributions, the required normalized input heat in a compression cycle can be calculated by integrating $\mathrm{d} q_{\text {input }}$ along the curve a-b-c in Figure 2.6,

$$
\begin{gathered}
\mathrm{d} q_{\text {input }}=\left(c_{\text {adsor }}+x_{a d s} c_{p, a d s}+x_{\text {fre }} c_{p, f r e}+\sum_{i} \frac{m_{\text {para }, i}}{m_{\text {sor }}} c_{\text {para }, i}\right) \mathrm{d} T \\
+\mathrm{d} q_{\text {sorp }}-h \mathrm{~d} x_{\text {tot }} \\
\tilde{q}_{\text {input }}=\left.\int_{a \rightarrow b} \mathrm{~d} q_{\text {input }}\right|_{x_{\text {tot }}}+\left.\int_{b \rightarrow c} \mathrm{~d} q_{\text {input }}\right|_{p}
\end{gathered}
$$

Here, the subscripted index "para,i" refers to a particular parasitic thermal mass component as discussed foregoing. Note that the total amount of the working fluid in the cell is constant from state "a" to "b", whereas the pressure is constant from "b" to "c".

Analogous to the expression of the COP for a single-stage compressor, a COP of the multistage sorption compressor can be defined as well. In stationary operation, the average generated flow rate $\dot{m}$ by each stage of a multistage compressor should be equal,

$$
\dot{m}=\frac{\tilde{m}_{1}}{t_{c y c, 1}}=\frac{\tilde{m}_{2}}{t_{c y c, 2}}=\ldots=\frac{\tilde{m}_{n}}{t_{c y c, n}}
$$

where the subscript $\mathrm{n}$ indicates the number of the compression stages. As a result, the overall COP for a n-stage sorption compressor is derived as,

$$
C O P_{S C, O A, n s}=\frac{\Delta \dot{G}}{\dot{Q}_{\text {input }}}=\frac{\Delta g \dot{m}}{\sum_{n} \dot{Q}_{\text {input }, i}}=\frac{\Delta g}{\sum_{n}\left(\tilde{Q}_{\text {input }, i} / \tilde{m}_{i}\right)}=\frac{\Delta g}{\sum_{n}\left(\tilde{q}_{\text {input }, i} / \tilde{x}_{i}\right)}
$$

where the input heat for each stage $\tilde{q}_{\text {input }, i}$ can be calculated by using Eq. 2.23 and it is reduced into Eq. 2.16 when $n=1$. 


\subsection{Working fluid optimization for the sorption-based Joule-Thomson cooler}

For a JT cooler operating at a specific cooling temperature, multiple working fluids usually can be chosen. In a previous study by Derking [65], the most suitable working fluid was selected considering the cold stage only. It was based on the coefficient of performance of an ideal JT cold stage and a figure of merit of the CFHX. This section presents a generalized methodology incorporating an overall coefficient of performance of the sorptionbased JT cooler $C O P_{O A}$ and the specific cooling energy in each compression cycle $q_{c}$. The questions aimed to answer here are: What fluid should be used in order to have the highest cooling energy per unit of heat put into the compressor, i.e. $C O P_{O A}$ ? And what, in that case, is the cooling energy per unit mass of the adsorbent material?

\subsubsection{Optimization criteria}

Two performance parameters are considered in selecting the best working fluid in a sorptionbased JT cooler:

\section{Overall coefficient of performance of a sorption-based JT cooler $\left(C O P_{O A}\right)$}

The overall coefficient of performance $C O P_{O A}$ of a sorption-based JT cooler is defined as the ratio of the heat taken from the load by the evaporator at the cold end to the total heat input in each compression cycle. Combining Eq. 2.11 and Eq. 2.25, the $C O P_{O A}$ of a basic JT cooler driven by an n-stage sorption compressor, can be written as the product of the COPs of the sorption compressor and the JT cold stage,

$$
C O P_{O A}=C O P_{J T C S} \cdot C O P_{S C, n s}=\frac{\Delta h_{c}}{\Delta g_{\text {compr }}} \frac{\Delta g_{\text {compr }}}{\sum_{n}\left(\tilde{q}_{\text {input }, i} / \tilde{x}_{i}\right)}=\frac{\Delta h_{c}}{\sum_{n}\left(\tilde{q}_{\text {input }, i} / \tilde{x}_{i}\right)}
$$

\section{Specific cooling energy in each compression cycle}

The specific cooling energy $q_{c}$ in a cycle is defined as the cooling energy per unit of mass of adsorbent:

$$
q_{c}=\frac{\tilde{m} \Delta h_{c}}{\sum_{n} m_{s o r, i}}=\left(\sum_{n} \frac{1}{\tilde{x}_{i}}\right)^{-1} \Delta h_{c}
$$

This is a useful parameter that provides insight into the mass efficiency of a specific combination of a working fluid and an adsorbent.

\subsubsection{Optimization method}

Each of the candidate fluids is considered separately, and the corresponding compressor operating parameters are optimized to achieve a maximum $C O P_{O A}$ for that specific fluid. Subsequently, the best fluid can be selected by comparing the respective $C O P_{O A}$ values. 
The loss terms taken into account are all related to the adsorbent and the fluids, i.e. energy lost in adsorbent heat capacity, gas lost in void volume, and the heat of adsorption. Therefore, the evaluated $C O P_{O A}$ indicates the maximum achievable performance. In practice, other loss terms, e.g., the parasitic thermal mass and the parasitic heat loads, will deteriorate the COP. Furthermore, we consider the total energies transferred and thus neglect any dynamic effect. Therefore, the COP evaluated in our method should primarily be used in a preliminary design phase for selecting the best working fluid. Once the fluid for a specific adsorbent material and specific temperature conditions has been selected, the compressor should be designed in more detail.

The optimization flowchart for a JT cooler driven by a single-stage sorption compressor is shown in Figure 2.9. In steps 1 and 2 the inputs are chosen: the cooling temperature $T_{c}$, the heat-sink temperature $T_{H S}$ and the adsorbent material. The cooling temperature determines what working fluids can be used and the corresponding boiling points determine the low pressure $p_{l}$ (point 1 in Figure 2.6 is thus fixed). For example, hydrogen and neon can be used to arrive at $30 \mathrm{~K}$, whereas nitrogen, carbon monoxide and methane can establish a temperature of $100 \mathrm{~K}$. In step 2, the selected gas is combined with an adsorben$\mathrm{t}$ material and the experimental isotherms (as plotted in Figure 2.6) and their theoretical correlations are important inputs.

For each sorption compressor, the high temperature $T_{h}$ and high pressure $p_{h}$ can be chosen in step 1 to determine the position and span of the cycle in the isotherm diagram, point "c" in Figure 2.6. Once points "a" and "c" of the cycle are fixed, the amount of pumped fluid and the heat input can be calculated per cycle in step 3 . The high pressure $p_{h}$ along with the heat-sink temperature $T_{H S}$ and the low pressure $p_{l}$ also determine the specific enthalpy difference of the working fluid at the warm end of the CFHX (step 4). The overall $C O P_{O A}$ of the system can now be evaluated using Eq. 2.26 (step 5). Within a practical pressure range, the high pressure $p_{h}$ is varied and for each value of $p_{h}$ the

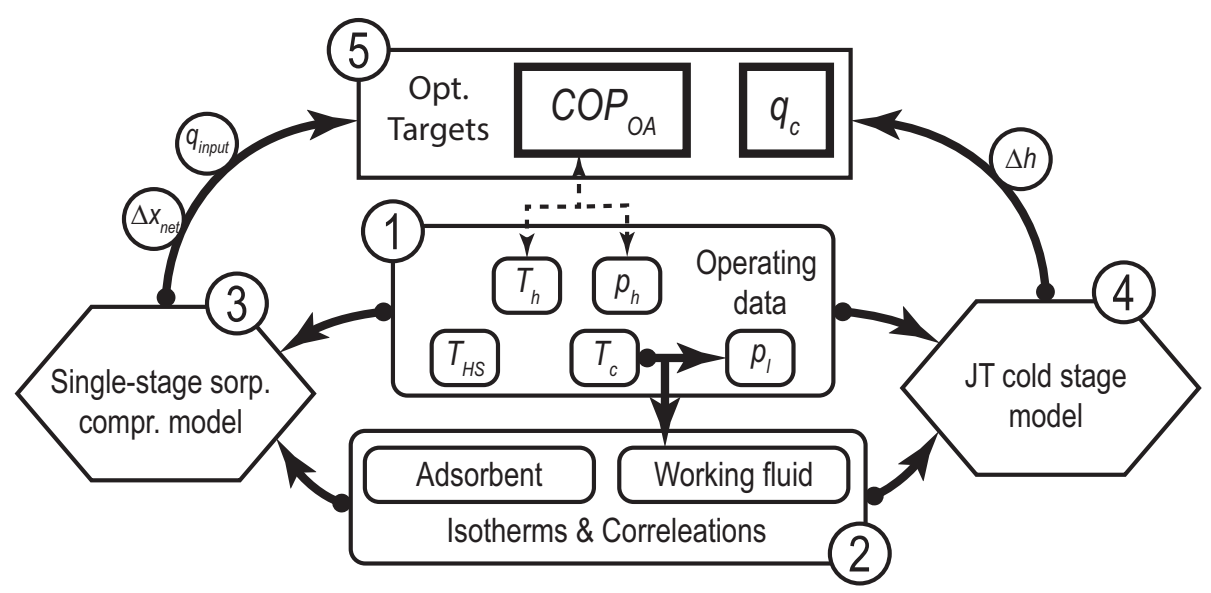

Figure 2.9. Optimization flowchart for a JT cooler driven by a single stage sorption compressor. 


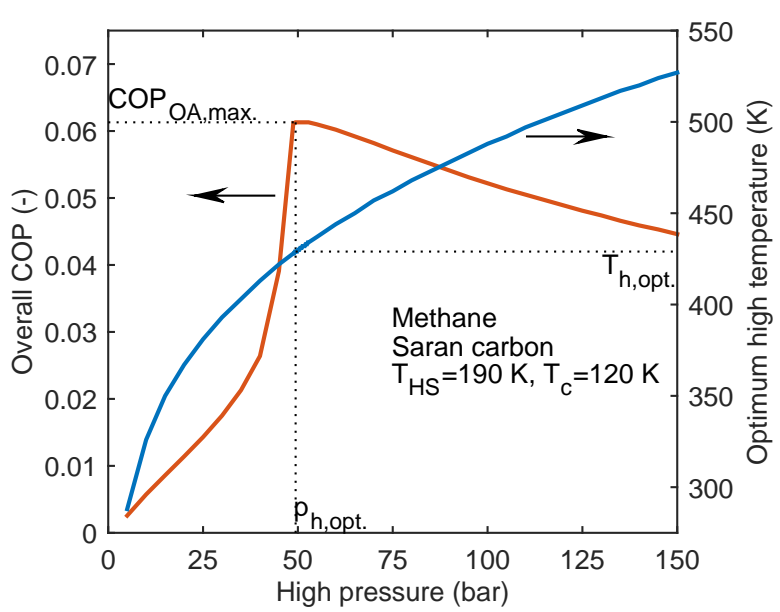

Figure 2.10. $C O P_{O A}$ and optimum $T_{h}$ as a function of $p_{h}$ for the combination methane and Saran carbon for a heat-sink temperature of $190 \mathrm{~K}$ and a cooling temperature of $120 \mathrm{~K}$.

optimum high temperature $T_{h}$ is determined. The corresponding values of $C O P_{O A}$ can now be plotted versus $p_{h}$ yielding the optimum parameters and maximum achievable COP. An example of such a plot is given in Figure 2.10.

The optimization process for a multistage sorption compressor is similar to that for the single-stage compressor. It is described here by taking a two-stage sorption compressor as an example. In a two-stage sorption compressor, the high pressure of the first stage, i.e. the intermediate pressure $p_{\text {im }}$ has to be determined, and the high temperatures for the two stages can be different. In order to find the optimum parameter combination, $p_{h}$ is fixed, and $p_{i m}, T_{h, 1}$ and $T_{h, 2}$ are optimized by using a multi-dimensional Newtons method. Repeating this calculation for different $p_{h}$, plots similar to Figure 2.10 can be created indicating the maximum achievable $C O P_{O A}$ and the optimum parameter values.

\subsubsection{Optimization constraints}

The analysis is based on the inherent properties of the fluids and the adsorbent, and sorption characteristics of the adsorbent-adsorbate pairs. The properties of the fluids are available from various fluid property software packages, such as Refprop [66]. The specific surface areas, specific heat capacities and the void volume fractions of the adsorbent materials are required in the sorption compressor modeling, and usually can be provided by the suppliers or are available in literature [67-69]. Also, the sorption isotherm data are crucial input as discussed in Sec. 2.2.2.

The operating temperature of the cooler is determined by the boiling point of the working fluid and thus by the pressure in the evaporator. In practice, the pressure drops due to viscous losses along the low-pressure line of the CFHX and the microchannels in the porous adsorbent make it difficult to realize a very low $p_{l}$. Therefore, in the optimiza- 
tion the value of $p_{l}$ is limited to the range of 0.2 bar to 10.0 bar. In case the triple point of a fluid is above 0.2 bar, the triple point pressure is chosen as the minimum low-pressure value. Defining the low-pressure range at specific operating temperatures obviously limits the number of candidate working fluids.

The heat-sink temperature $T_{H S}$ of the sorption compressor, which in this optimization is equal to the warm-end temperature of the JT cold stage, affects $C O P_{O A}$ significantly. Because of the higher adsorption capacity of the adsorbent materials at a lower temperature, sorption compressors are usually operated at a minimum $T_{H S}$ in order to achieve high efficiency. Typical temperature levels can be selected for specific applications, for example, an ambient temperature of $293 \mathrm{~K}$ for a nitrogen cooler for cooling a groundbased IR sensor, or a cryogenic temperature of $60 \mathrm{~K}$ maintained by a radiator for a $5 \mathrm{~K}$ helium cooler for space application [70].

The operating high pressure $p_{h}$ and the high temperature $T_{h}$ are two intermediate variables to be optimized in finding the maximum $C O P_{O A}$ for a specific working fluid. The maximum high pressure $p_{h}$ is set at 150 bar as a practical limitation resulting from the compressor realization. The high temperature $T_{h}$ should not be too high relative to the heat-sink temperature $T_{H S}$ in order to limit the entropy production (and thus the losses) when the sorption cells are cooling down. Therefore, a high temperature limitation has to be selected based on the heat-sink temperature.

\subsubsection{Discussion on optimization results}

Optimization results are presented here for carbonized saran. It has a high specific surface area of more than $1000 \mathrm{~m}^{2} / \mathrm{g}$ and its high adsorption capacity and relatively low porosity make it an excellent adsorbent to be applied in a sorption compressor [68].

The working fluids that are considered in this investigation are listed in Figure 2.11 along with their operating temperature ranges based on our low-pressure setting discussed in Sec. 2.3.3. Two temperature ranges result: $16-38 \mathrm{~K}$ and $65-160 \mathrm{~K}$. In the gap from

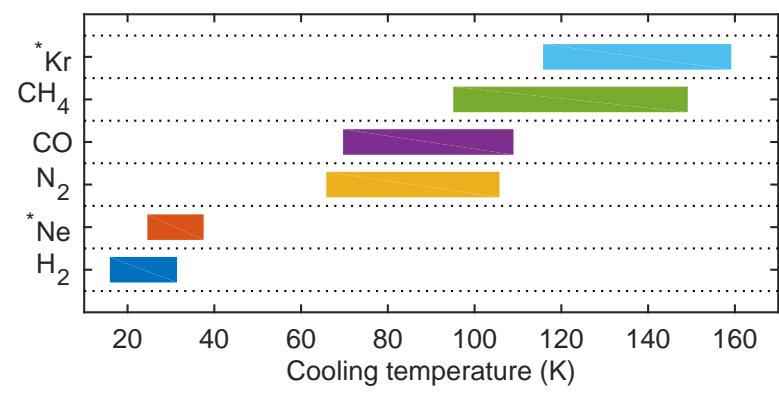

Figure 2.11. Candidate working fluids for a sorption-based JT cooler corresponding to a lowpressure range of 0.2-10 bar. *Triple point pressure is used to calculate the minimum temperature for krypton and neon. 
Table 2.2. List of selected values of $T_{H S}$ for working fluids and max. $T_{h}$.

\begin{tabular}{cccccccc}
\hline$T_{H S} / \mathrm{K}$ & $\mathrm{Kr}$ & $\mathrm{CH}_{4}$ & $\mathrm{CO}$ & $\mathrm{N}_{2}$ & $\mathrm{Ne}$ & $\mathrm{H}_{2}$ & Max. $T_{h} / \mathrm{K}$ \\
\hline 293 & $\bullet$ & $\bullet$ & $\bullet$ & $\bullet$ & & & 700 \\
190 & $\bullet$ & $\bullet$ & $\bullet$ & $\bullet$ & & & 530 \\
150 & $\bullet$ & $\bullet$ & $\bullet$ & $\bullet$ & $\bullet$ & $\bullet$ & 450 \\
120 & & & & & $\bullet$ & $\bullet$ & 370 \\
77 & & & & & $\bullet$ & $\bullet$ & 280 \\
\hline
\end{tabular}

38-65 K, no pure working fluid liquefies within the selected low-pressure range. A temperature below $15 \mathrm{~K}$ in a JT cooler can only be realized with helium and below about $5 \mathrm{~K}$, a selection should be made between helium-3 and helium-4, but that is beyond the scope of this thesis.

Typical heat-sink temperature levels are selected for specific working fluids, as listed in Table 2.2. For hydrogen and neon whose maximum inversion temperatures are below room temperature, lower heat-sink temperatures are chosen. At each heat-sink temperature, a limit is set to the high temperature $T_{h}$ and included in Table 2.2.

\section{Results for single-stage compression}

Results for a JT cooler driven by a single-stage sorption compressor are presented in this section. The temperature range of $65-160 \mathrm{~K}$ is covered by nitrogen, carbon monoxide, krypton, and methane. Figure 2.12 shows the $C O P_{O A}$ for these fluids as a function of the high pressure for $T_{c}$ equals: (a) $120 \mathrm{~K}$, (b) $100 \mathrm{~K}$, and (c) $77 \mathrm{~K}$. The optimum parameters and corresponding performance of the cooler are shown in Table 2.3. The $C O P_{O A}$ is relatively poor when the heat-sink temperature is at room temperature, $293 \mathrm{~K}$. At room temperature, all fluids are supercritical and behave essentially as an ideal gas. Therefore, only small changes in specific enthalpy can be established under compression. As a result, the COP of the JT cold stage will be quite low. In addition, saran has a low adsorption capacity for the working fluids at high heat-sink temperature. In that case, the sorbent has to be heated up to a very high temperature to build up a high pressure in the cell, which results in a large compressor heat input and a rather small amount of fluid transferred during a cycle. Thus the $C O P_{S C}$ is typically lower than $0.2 \%$, as shown in Table 2.3. A lower heat-sink temperature significantly improves the performance of a sorption-based JT cooler and decreases the optimum $T_{h}$ and $p_{h}$. Also, the specific cooling energy $q_{c}$ increases, as listed in Table 2.3, which means that much less adsorbent material is needed. Therefore, in practice, the mass and volume of the system which is proportional to the amount of the adsorbent can be reduced remarkably by lowering $T_{H S}$.

Close to its critical temperature, a fluid behaves highly non-ideal, and compression results in a much higher change in specific enthalpy. A steep increase occurs in the $C O P_{J T C S}$ at a specific high pressure, as well as in the $C_{O P}$ (Figure 2.12 (a) for krypton and methane). 


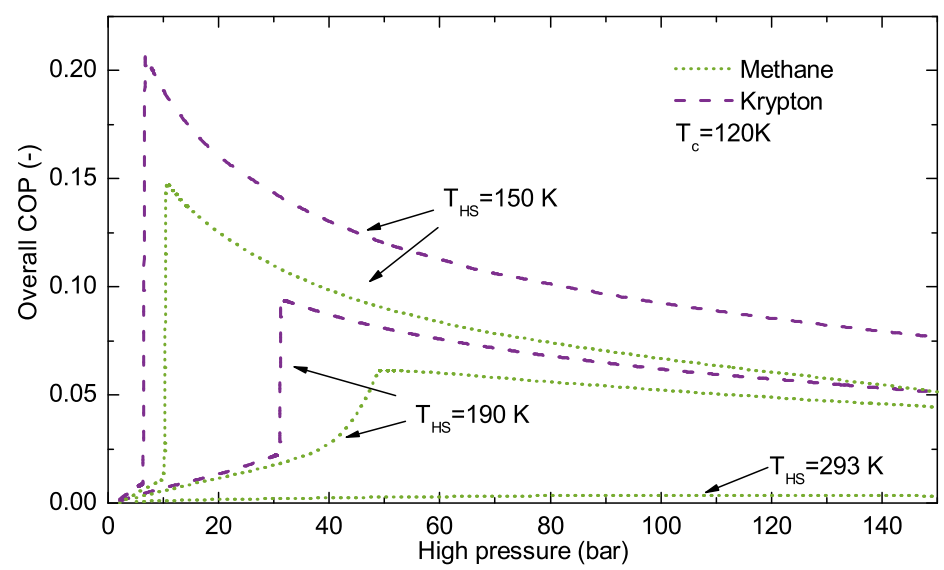

(a) $T_{c}=120 \mathrm{~K}$

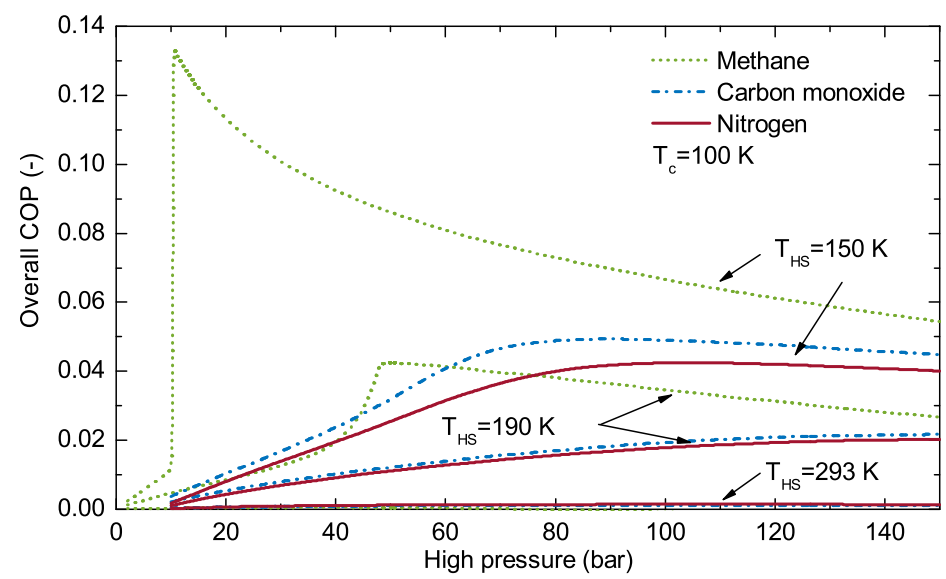

(b) $T_{c}=100 \mathrm{~K}$

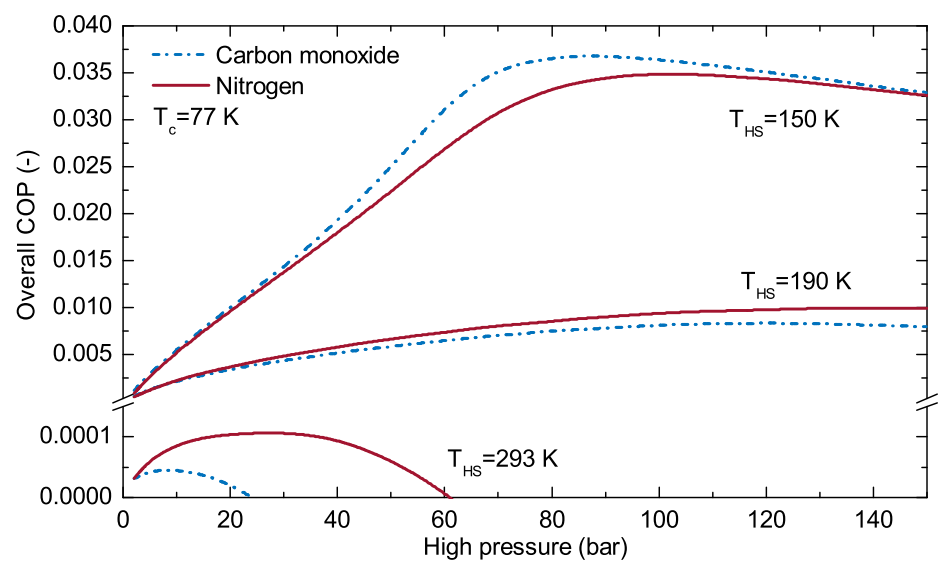

(c) $T_{c}=77 \mathrm{~K}$

Figure 2.12. $C O P_{O A}$ as a function of the high pressure for three values of $T_{c}$. 
Table 2.3. Max. $C O P_{O A}$ parameters for a sorption-based JT cooler (single-stage compressor), $T_{c}=120 \mathrm{~K}, 100 \mathrm{~K}, 77 \mathrm{~K}$.

\begin{tabular}{|c|c|c|c|c|c|c|c|c|}
\hline Fluid & & $\begin{array}{c}p_{l} \\
\text { (bar) }\end{array}$ & $\begin{array}{c}p_{h, o p t} \\
\text { (bar) }\end{array}$ & $C O P_{O A, \max }$ & $C O P_{S C}$ & $C O P_{J T C S}$ & $\begin{array}{c}T_{h, o p t} \\
(\mathrm{~K})\end{array}$ & $\begin{array}{c}q_{c} \\
(\mathrm{~kJ} / \mathrm{kg})\end{array}$ \\
\hline \multicolumn{9}{|c|}{ (a) $T_{c}=120 \mathrm{~K}$} \\
\hline \multirow{2}{*}{$T_{H S}=293 \mathrm{~K}$} & $\mathrm{Kr}$ & 1.034 & 65.0 & 0.00173 & 0.0139 & 0.124 & 700 & 0.928 \\
\hline & $\mathrm{CH}_{4}$ & 1.914 & 115.0 & 0.00366 & 0.0182 & 0.201 & 700 & 2.170 \\
\hline \multirow{2}{*}{$T_{H S}=190 \mathrm{~K}$} & $\mathrm{Kr}$ & 1.034 & 31.2 & 0.09420 & 0.0676 & 1.390 & 403 & 26.88 \\
\hline & $\mathrm{CH}_{4}$ & 1.914 & 49.4 & 0.06130 & 0.0512 & 1.200 & 429 & 21.43 \\
\hline \multirow{2}{*}{$T_{H S}=150 \mathrm{~K}$} & $\mathrm{Kr}$ & 1.034 & 6.6 & 0.20800 & 0.0551 & 3.780 & 286 & 41.55 \\
\hline & $\mathrm{CH}_{4}$ & 1.914 & 10.4 & 0.14881 & 0.0402 & 3.699 & 311 & 35.53 \\
\hline \multicolumn{9}{|c|}{ (b) $T_{c}=100 \mathbf{K}$} \\
\hline \multirow{3}{*}{$T_{H S}=293 \mathrm{~K}$} & $\mathrm{CH}_{4}$ & 0.344 & 35.0 & 0.00055 & 0.0107 & 0.0517 & 700 & 0.292 \\
\hline & $\mathrm{CO}$ & 5.444 & 110.0 & 0.00112 & 0.0118 & 0.0949 & 700 & 0.615 \\
\hline & $\mathrm{N}_{2}$ & 7.783 & 110.0 & 0.00144 & 0.0161 & 0.0894 & 618 & 0.618 \\
\hline \multirow{3}{*}{$T_{H S}=190 \mathrm{~K}$} & $\mathrm{CH}_{4}$ & 0.344 & 50.4 & 0.04237 & 0.0561 & 0.7556 & 498 & 17.82 \\
\hline & $\mathrm{CO}$ & 5.444 & 150.0 & 0.02169 & 0.0433 & 0.5007 & 426 & 6.91 \\
\hline & $\mathrm{N}_{2}$ & 7.783 & 150.0 & 0.02030 & 0.0430 & 0.4720 & 414 & 6.37 \\
\hline \multirow{3}{*}{$T_{H S}=150 \mathrm{~K}$} & $\mathrm{CH}_{4}$ & 0.344 & 10.4 & 0.13352 & 0.0745 & 1.7920 & 335 & 34.25 \\
\hline & $\mathrm{CO}$ & 5.444 & 90.0 & 0.04939 & 0.0409 & 1.2083 & 339 & 13.71 \\
\hline & $\mathrm{N}_{2}$ & 7.783 & 105.0 & 0.04242 & 0.0370 & 1.1459 & 348 & 12.61 \\
\hline \multicolumn{9}{|c|}{ (b) $T_{c}=77 \mathbf{K}$} \\
\hline \multirow{2}{*}{$T_{H S}=293 \mathrm{~K}$} & $\mathrm{CO}$ & 0.577 & 10.0 & 0.00005 & 0.00439 & 0.0104 & 617 & 0.017 \\
\hline & $\mathrm{N}_{2}$ & 0.972 & 25.0 & 0.00011 & 0.00551 & 0.0194 & 654 & 0.046 \\
\hline \multirow{2}{*}{$T_{H S}=190 \mathrm{~K}$} & $\mathrm{CO}$ & 0.577 & 120.0 & 0.00800 & 0.0310 & 0.2660 & 530 & 3.32 \\
\hline & $\mathrm{N}_{2}$ & 0.972 & 140.0 & 0.00992 & 0.0366 & 0.2715 & 530 & 4.24 \\
\hline \multirow{2}{*}{$T_{H S}=150 \mathrm{~K}$} & $\mathrm{CO}$ & 0.577 & 85.0 & 0.03680 & 0.0602 & 0.6110 & 398 & 11.40 \\
\hline & $\mathrm{N}_{2}$ & 0.972 & 100.0 & 0.03484 & 0.0588 & 0.5927 & 394 & 11.05 \\
\hline
\end{tabular}


Table 2.4. Optimum working fluids and temperature range depending on compressor heat-sink temperature.

\begin{tabular}{ccccc}
\hline \multicolumn{5}{c}{$T_{c}(\mathrm{~K})$} \\
\hline$T_{H S}(\mathrm{~K})$ & $\mathrm{N}_{2}$ & $\mathrm{CO}$ & $\mathrm{CH}_{4}$ & $\mathrm{Kr}$ \\
150 & $65-70$ & $70-95$ & $95-116$ & $116-140$ \\
190 & $65-90$ & $90-95$ & $95-116$ & $116-160$ \\
293 & $65-104$ & $104-109$ & $109-150$ & $150-160$ \\
${ }^{\text {Cold stage only [65 }{ }^{a}}$ & $65-70$ & $70-95^{b}$ & $95-116$ & $116-140^{c}$ \\
\hline${ }^{a}$ Inlet of the cold stage at 300 K [65] & & \\
${ }^{b}$ Result in [65] for oxygen \\
${ }^{c}$ Result in [65] for nitrogen-trifluoride
\end{tabular}

These calculations and considerations are made for multiple cooling temperatures. Then, the temperature range of $65-160 \mathrm{~K}$ can be divided into subranges, and optimum working fluids can be selected based on the maximum $C O P_{O A}$ and corresponding $q_{c}$. At the low-temperature end of the range, nitrogen is the best choice, whereas at higher temperatures, successively carbon monoxide, methane and krypton become better. The results are shown in Figure 2.13 and summarized in Table 2.4. In this table the optimization results are included that are based on the performance of the cold stage only, as presented in the study of Derking et al [65].

In that study, Derking et al. included oxygen and nitrogen-trifluoride as indicated in Table 2.4. Because of material oxidation and toxicity we have not included these gasses in our analysis. Nevertheless, it is striking to see that the results correspond quite well to the low compressor heat-sink temperatures, whereas at $T_{H S}=293 \mathrm{~K}$ including the sorption compressor in the analysis shifts the optimum-gas ranges to higher temperatures.

This effect can best be explained by considering nitrogen and methane aiming at a cold-tip temperature of $100 \mathrm{~K}$ starting from a warm end at $293 \mathrm{~K}$. If only the cold stage is considered, then Derking et al. find that both gases should be operated at the maximum available pressure considered in their study of 200 bar, and that in that case methane has almost a factor of 2 better cold-stage COP. However, when including a sorption compressor Figure 2.12 shows that things change significantly. At $293 \mathrm{~K}$, the attainable high pressure for methane, constrained by the maximum sink temperature of $700 \mathrm{~K}$, is only 35 bar resulting in a much lower cold-stage COP. Nitrogen can be operated at an optimum setting, sink temperature $618 \mathrm{~K}$, and high pressure of 110 bar. This is significantly higher than in the methane case. As a result in Figure 2.12, the cold-stage COP of nitrogen is better than that of methane. In addition, the compressor COP of nitrogen is better as well. In order to have a boiling point at $100 \mathrm{~K}$, the low pressure in the methane system should be 0.344 bar whereas nitrogen can operate with a low pressure of 7.783 bar. However, the total amount of gas in the compressor at the starting point of the compression cycle for methane is much smaller than that for nitrogen $(15.8 \mathrm{~g} / \mathrm{kg}$ vs. $66.6 \mathrm{~g} / \mathrm{kg})$. Therefore, it is harder to establish a high pressure in methane, certainly when starting from a lower pres- 


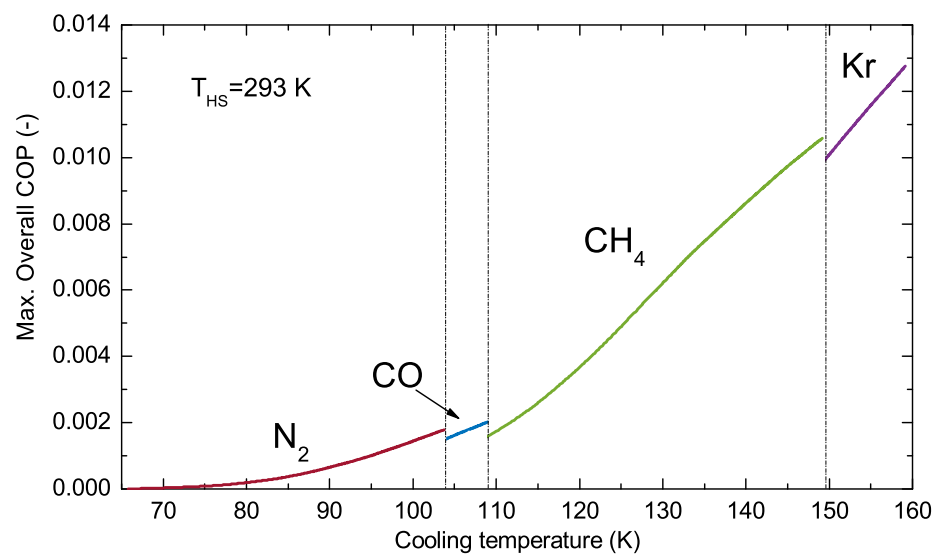

(a) $T_{H S}=293 \mathrm{~K}$

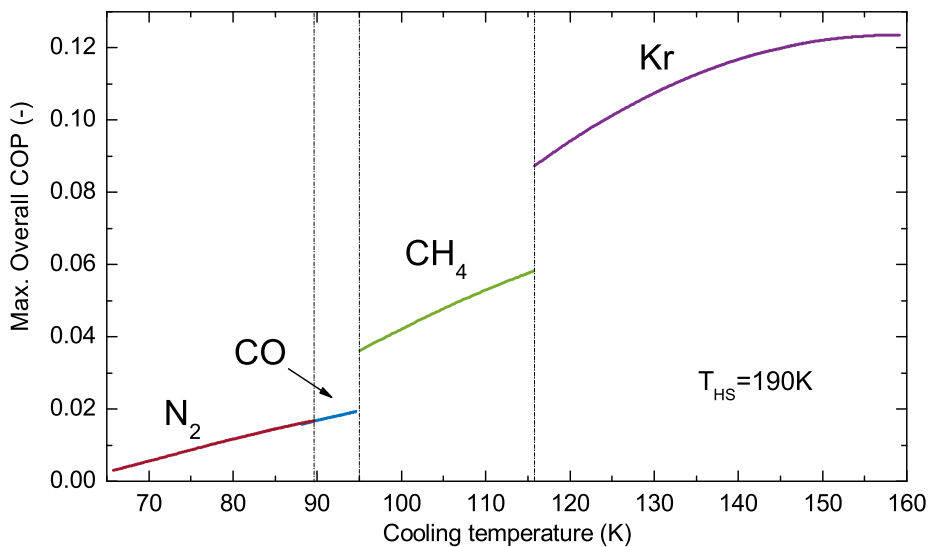

(b) $T_{H S}=190 \mathrm{~K}$

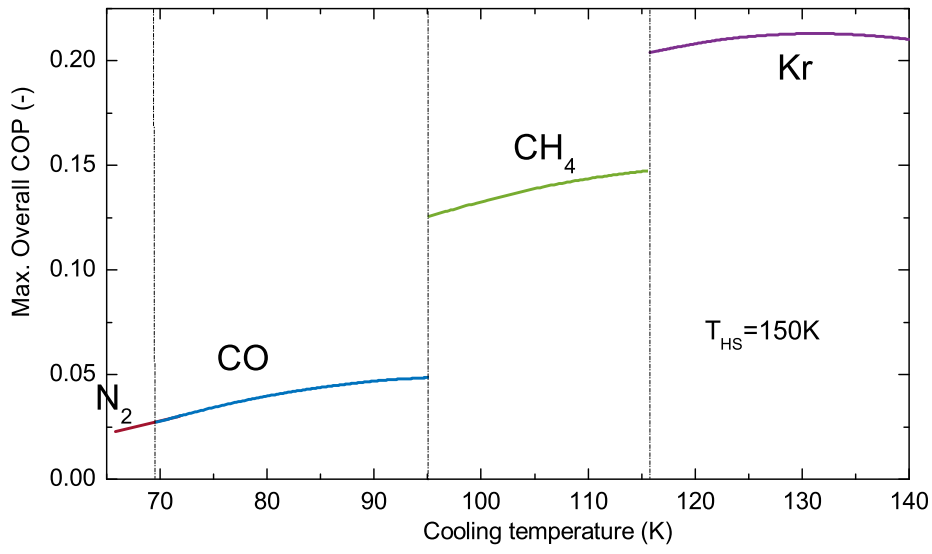

(c) $T_{H S}=150 \mathrm{~K}$

Figure 2.13. Max. $C O P_{O A}$ as a function of the cooling temperature for three $T_{H S}$. 
Table 2.5. Maximum $C O P_{O A}$ parameters for a sorption-based JT cooler operating with neon or hydrogen (single-stage compressor), $T_{c}=30 \mathrm{~K}$.

\begin{tabular}{ccccccccc}
\hline Fluid & $\begin{array}{c}p_{l} \\
\text { (bar) }\end{array}$ & $\begin{array}{c}p_{h, \text { opt }} \\
\text { (bar) }\end{array}$ & COP OA,max. & COPSC $_{S C}$ & COP $_{\text {JTCS }}$ & $\begin{array}{c}T_{h, o p t} \\
(\mathrm{~K})\end{array}$ & $\begin{array}{c}q_{c} \\
(\mathrm{~kJ} / \mathrm{kg})\end{array}$ \\
\hline (a) $T_{c}=30 \mathrm{~K}$ & & & & & & & \\
\hline \multirow{2}{*}{$T_{H S}=150 \mathrm{~K}$} & $\mathrm{Ne}$ & 2.231 & 13.0 & $1.809 \mathrm{E}-5$ & 0.00307 & 0.00589 & 356 & 2.343 \\
& $\mathrm{H}_{2}$ & 8.043 & 35.0 & $4.271 \mathrm{E}-4$ & 0.03649 & 0.01171 & 278 & 38.12 \\
\hline \multirow{2}{*}{$T_{H S}=120 \mathrm{~K}$} & $\mathrm{Ne}$ & 2.231 & 25.0 & $1.868 \mathrm{E}-4$ & 0.00908 & 0.02057 & 328 & 22.84 \\
& $\mathrm{H}_{2}$ & 8.043 & 65.0 & $2.316 \mathrm{E}-3$ & 0.05622 & 0.04119 & 268 & 264.1 \\
\hline \multirow{2}{*}{$T_{H S}=77 \mathrm{~K}$} & $\mathrm{Ne}$ & 2.231 & 105.0 & $1.212 \mathrm{E}-2$ & 0.05749 & 0.21077 & 242 & 1358 \\
& $\mathrm{H}_{2}$ & 8.043 & 105.0 & $1.554 \mathrm{E}-2$ & 0.07641 & 0.20342 & 204 & 1839 \\
\hline
\end{tabular}

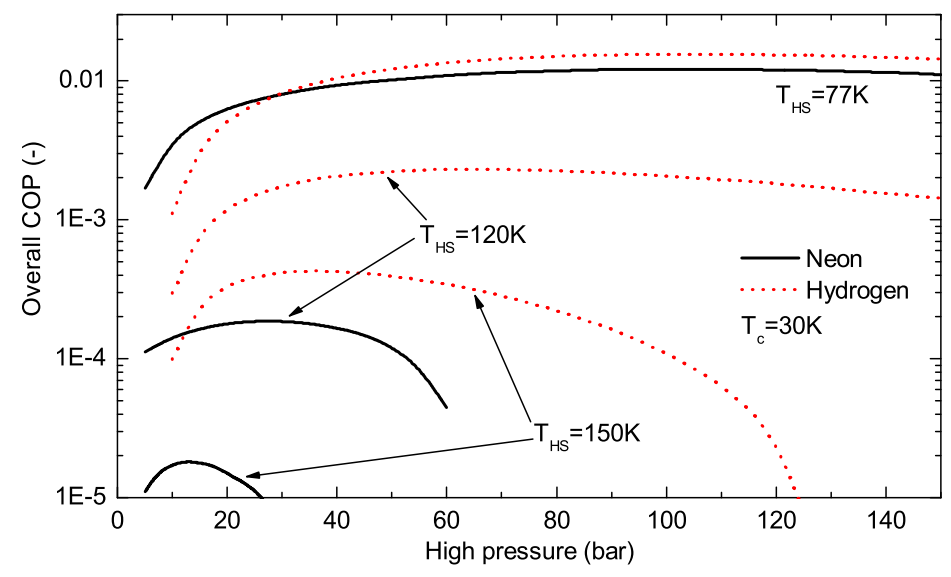

Figure 2.14. $C O P_{O A}$ as a function of the high pressure for $T_{c}$ of $30 \mathrm{~K}$.

sure. As indicated in Figure 2.12, the compression ratio in the methane system at $293 \mathrm{~K}$ is 102 whereas in nitrogen it is only 14 , much easier to achieve. The energy input to the compressor can be related to the required temperature increase. In the methane compressor at $293 \mathrm{~K}$ this would be $407 \mathrm{~K}$ to realize 35 bar, whereas the nitrogen compressor can establish 110 bar with a temperature increase of "only" $325 \mathrm{~K}$. All these effects result for methane in a lower compressor COP that, combined with the lower cold-stage COP, result in a lower overall performance.

In the temperature range of 16-38 K, neon and hydrogen can be used as the working fluids. As a result of the low-pressure range selection, neon can be used in the temperature range of $25-38 \mathrm{~K}$ and hydrogen in the range of $16-31 \mathrm{~K}$. In the range $25-31 \mathrm{~K}$ both fluids need to be compared. For example, for a cooling temperature of $30 \mathrm{~K}$, the optimum parameters and the corresponding performance of the cooler are calculated and listed in Table 2.5. In Figure 2.14, the $C O P_{O A}$ values as a function of high pressure are 


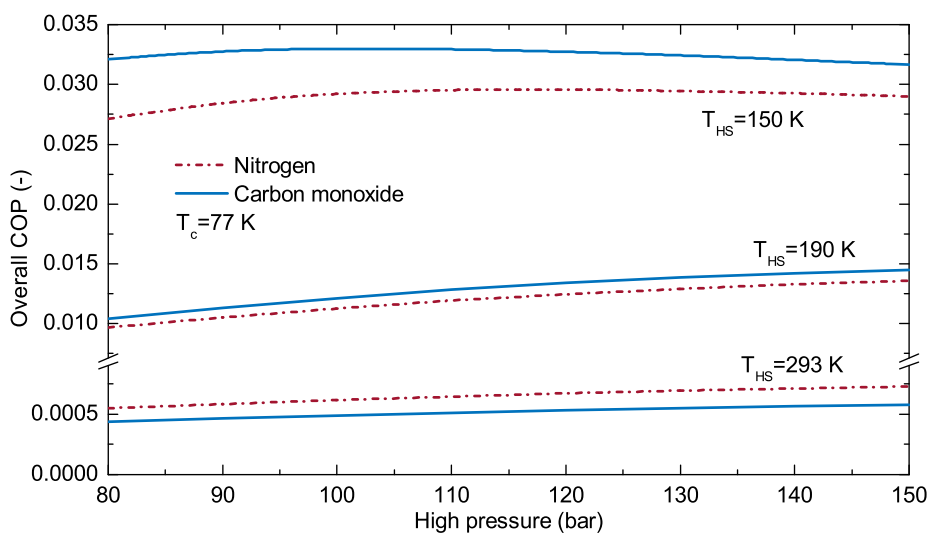

Figure 2.15. $\mathrm{COP}_{O A}$ as a function of the high pressure for JT cooler with two-stage sorption compressor, $T_{c}=77 \mathrm{~K}$.

plotted. From the previous study of Derking et al [65] it was not clear which working fluid could best be used in this cooling temperature range. However, when including a sorption compressor in the analysis, hydrogen appears to have a better performance than neon for a cooling temperature of $30 \mathrm{~K}$ as shown in Table 2.5. At a specific evaporator temperature, the low pressure in the hydrogen system is always higher than that in the neon system. Therefore, a hydrogen sorption compressor can establish a higher optimum high pressure with a lower high temperature requiring less heat input. In addition, hydrogen with a higher optimum high pressure gives larger $q_{c}$ values than neon (10 times at $30 \mathrm{~K})$. Consequently hydrogen is the best choice, and this statement holds true in the whole overlapping region $(25-31 \mathrm{~K})$ and is not affected by the heat-sink temperature.

\section{Results for two-stage compression}

Some illustrative results for a JT cooler driven by a two-stage sorption compressor are shown in Figure 2.15. The optimum $p_{h}, p_{i}, T_{h, 1}$ and $T_{h, 2}$ at maximum overall COP, and the corresponding cooler performance are shown in Table 2.6. Compared to the results for a single-stage compressor in Table 2.3, the $C O P_{O A}$ and $q_{c}$ both benefit from the two-stage arrangement if the heat-sink temperature is $293 \mathrm{~K}$. However, as the heat-sink temperature decreases and the adsorption capacity of the carbon increases, the optimum $p_{h}$ as well as the pressure ratio, tend to become smaller. As a result, using a two-stage compressor becomes less advantageous $\left(T_{H S}=190 \mathrm{~K}\right)$ or even worse $\left(T_{H S}=150 \mathrm{~K}\right)$.

In order to determine when to use a single-stage compressor and when a two stage, a contour map of constant maximum $C O P_{O A}$ values as a function of $T_{c}$ and $T_{H S}$ can be made, as shown in Figure 2.16 for nitrogen. The dashed line is a boundary line that separates the map into two parts: below the line, two-stage compression yields a higher $C O P_{O A}$ because of the required higher pressure ratio due to lower $T_{c}$ and higher $T_{H S}$; above the line, single-stage compression. The boundary line provides a useful criterion 
Table 2.6. Maximum overall COP parameters for a sorption-based JT cooler (two stage compressor), $T_{c}=77 \mathrm{~K}$.

\begin{tabular}{|c|c|c|c|c|c|c|c|c|c|c|}
\hline Fluid & $\begin{array}{l}T_{H S} \\
(\mathrm{~K})\end{array}$ & $\begin{array}{c}p_{l} \\
\text { (bar) }\end{array}$ & $\begin{array}{c}p_{\text {im,opt }} \\
\text { (bar) }\end{array}$ & $\begin{array}{l}p_{h, o p t} \\
\text { (bar) }\end{array}$ & $C O P_{O A, \max }$ & $C O P_{S C}$ & $C O P_{J T C S}$ & $\begin{array}{c}T_{h, 1, o p t} \\
(\mathrm{~K})\end{array}$ & $\begin{array}{c}T_{h, 1, o p t} \\
(\mathrm{~K})\end{array}$ & $\begin{array}{c}q_{c} \\
(\mathrm{~kJ} / \mathrm{kg})\end{array}$ \\
\hline $\mathrm{CO}$ & \multirow{2}{*}{293} & 0.577 & 6.0 & 150.0 & $\begin{array}{c}0.00058 \\
(0.00005)^{a}\end{array}$ & 0.0084 & 0.069 & 537 & 750 & $\begin{array}{c}0.205 \\
(0.017)\end{array}$ \\
\hline $\mathrm{N}_{2}$ & & 0.972 & 9.2 & 150.0 & $\begin{array}{c}0.00073 \\
(0.00011)\end{array}$ & 0.0113 & 0.064 & 505 & 659 & $\begin{array}{c}0.214 \\
(0.046)\end{array}$ \\
\hline $\mathrm{CO}$ & \multirow{2}{*}{190} & 0.577 & 7.4 & 150.0 & $\begin{array}{c}0.01451 \\
(0.00800)\end{array}$ & 0.0492 & 0.295 & 333 & 411 & $\begin{array}{c}3.09 \\
(3.32)\end{array}$ \\
\hline $\mathrm{N}_{2}$ & & 0.972 & 9.4 & 150.0 & $\begin{array}{c}0.01358 \\
(0.00992)\end{array}$ & 0.0486 & 0.280 & 324 & 405 & $\begin{array}{c}2.88 \\
(4.24)\end{array}$ \\
\hline $\mathrm{CO}$ & \multirow{2}{*}{150} & 0.577 & 3.2 & 100.0 & $\begin{array}{c}0.03303 \\
(0.03680)\end{array}$ & 0.0521 & 0.634 & 247 & 353 & $\begin{array}{c}6.73 \\
(11.40)\end{array}$ \\
\hline $\mathrm{N}_{2}$ & & 0.972 & 4.0 & 120.0 & $\begin{array}{c}0.02959 \\
(0.03484)\end{array}$ & 0.0481 & 0.615 & 237 & 367 & $\begin{array}{c}6.12 \\
(11.05)\end{array}$ \\
\hline
\end{tabular}

${ }^{a}$ Numbers in the brackets are the values of a cooler driven by the single-stage compressor, referred to Table 2.3

for preliminary design purposes. The boundary line can fairly well be approximated by,

$$
T_{c}=0.35 T_{H S}+21
$$

If the cold-tip temperature is below the value resulting from this equation, then twostage compression should be considered. For instance, starting from a sink temperature of $220 \mathrm{~K}$, two-stage compression is attractive if the target tip temperature is be below $87 \mathrm{~K}$.

\subsection{Conclusions}

Basic and precooled JT cold stages are thermally analyzed based on ideal performance. The sorption compression cycle is introduced, as well as the comparison of physisorption and chemisorption and the thermal properties of the adsorbed fluid. Then a lumped, static analysis is performed on a single-stage sorption compressor.

Based on the thermodynamic analysis of the JT cold stage and the sorption compressor, a general methodology for optimizing the working fluid in a sorption-based JT cooler is presented. By using this method, the working fluid of a JT cooler driven by a singlestage sorption compressor is optimized for two ranges of cold-tip operating temperatures: $65-160 \mathrm{~K}$ and $16-38 \mathrm{~K}$. In the former range, nitrogen is best at the low-temperature end, followed by carbon monoxide, methane, and krypton. The boundary temperatures depend on the heat-sink temperature. For instance, starting from $293 \mathrm{~K}$, the respective boundaries 


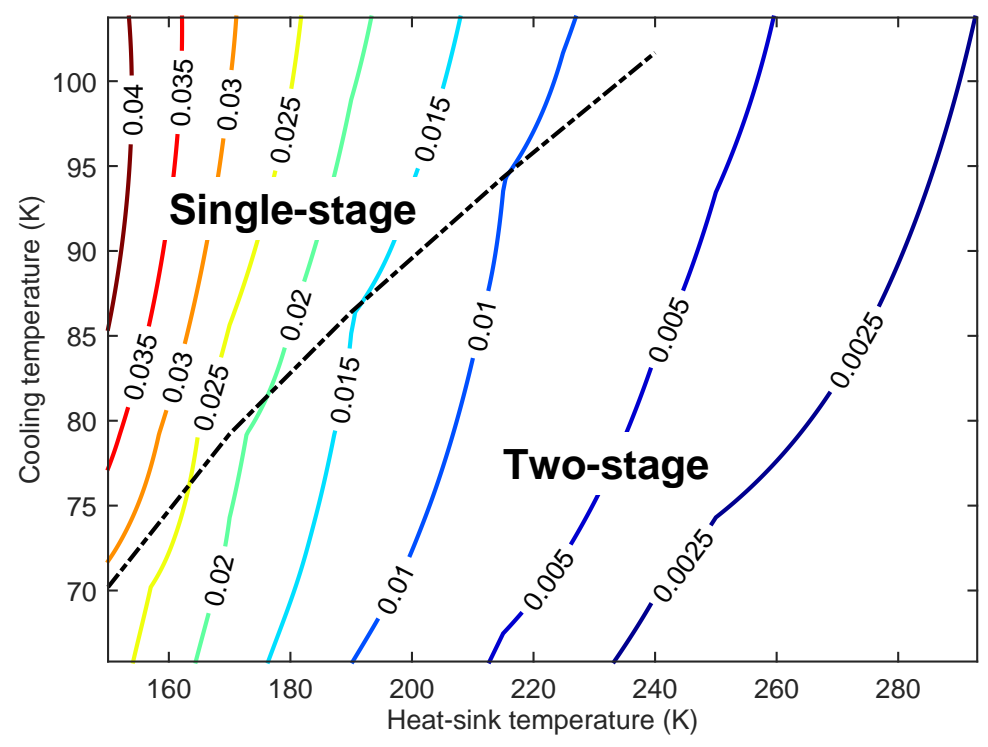

Figure 2.16. Max. $C O P_{O A}$ map for nitrogen when both single-stage and two-stage compression is considered (see text).

are 65-N2-104-CO-109-CH4-150-Kr-160. In the lower temperature range hydrogen is preferred up to $31 \mathrm{~K}$ and above that temperature neon is better.

The optimization method is also extended to two-stage compression, and specifically nitrogen and carbon monoxide are considered. A useful criterion for preliminary design purposes was derived indicating that, for nitrogen as the working fluid, two-stage compression is preferred if the cold-tip temperature is below $T_{c}=0.35 T_{H S}+21 \mathrm{~K}$, in which $T_{c}$ is the heat-sink temperature. The same method can be applied for other working fluids to find a maximum $C O P_{O A}$. Combined with the working fluid optimization method, the best working fluid can be found when both single stage compression and two-stage compression are taken into account. 


\section{Baseline Design of the METIS Cooler}

This chapter introduces the baseline cooler design for the METIS instrument. The baseline design should give a first impression of the system level configuration, performance, and size. Based on the thermodynamic analysis presented in Chapter 2, a quasi-static model for the sorption JT cooler was built. With proper presumptions and design margin$\mathrm{s}$, this model was used to optimize the system operating parameters such as the working pressures, the heating temperatures, etc.. 


\subsection{METIS cooler chain}

Based on our experience with sorption-based coolers, we designed a sorption JT cooler chain that meets the requirements of the METIS instrument [71]. As shown in Figure 3.1, this conceptual design consists of three stages thermally linked in parallel, to obtain cooling at $40 \mathrm{~K}, 25 \mathrm{~K}$ and $8 \mathrm{~K}$ individually. Neon, hydrogen, and helium are selected as the working fluids for three stages respectively, based on the optimization described in Sec. 2.3.

The helium stage is driven by a single-stage sorption compressor unit and uses four CFHXs, followed by a Joule-Thomson Restriction (JTR) and a cold-tip heat exchanger. In order to facilitate the maximum achievable performance, pre-cooling heat exchangers are applied at $40 \mathrm{~K}, 25 \mathrm{~K}$ and $15 \mathrm{~K}$. The efficiency of the helium stage is maximized by reducing the precooling temperature as far as possible. The limiting factor in this respect is that the pressure drop in the hydrogen-stage return line becomes more significant compared to the lower boiling-point pressure that corresponds to the reduced evaporator temperature. At $15 \mathrm{~K}$ the boiling pressure of hydrogen is 0.13 bar that we consider a minimum. Furthermore, at lower temperatures the hydrogen in the high-pressure line has a higher risk of freezing. Extensive experience on a $15 \mathrm{~K}$ hydrogen sorption cooler was

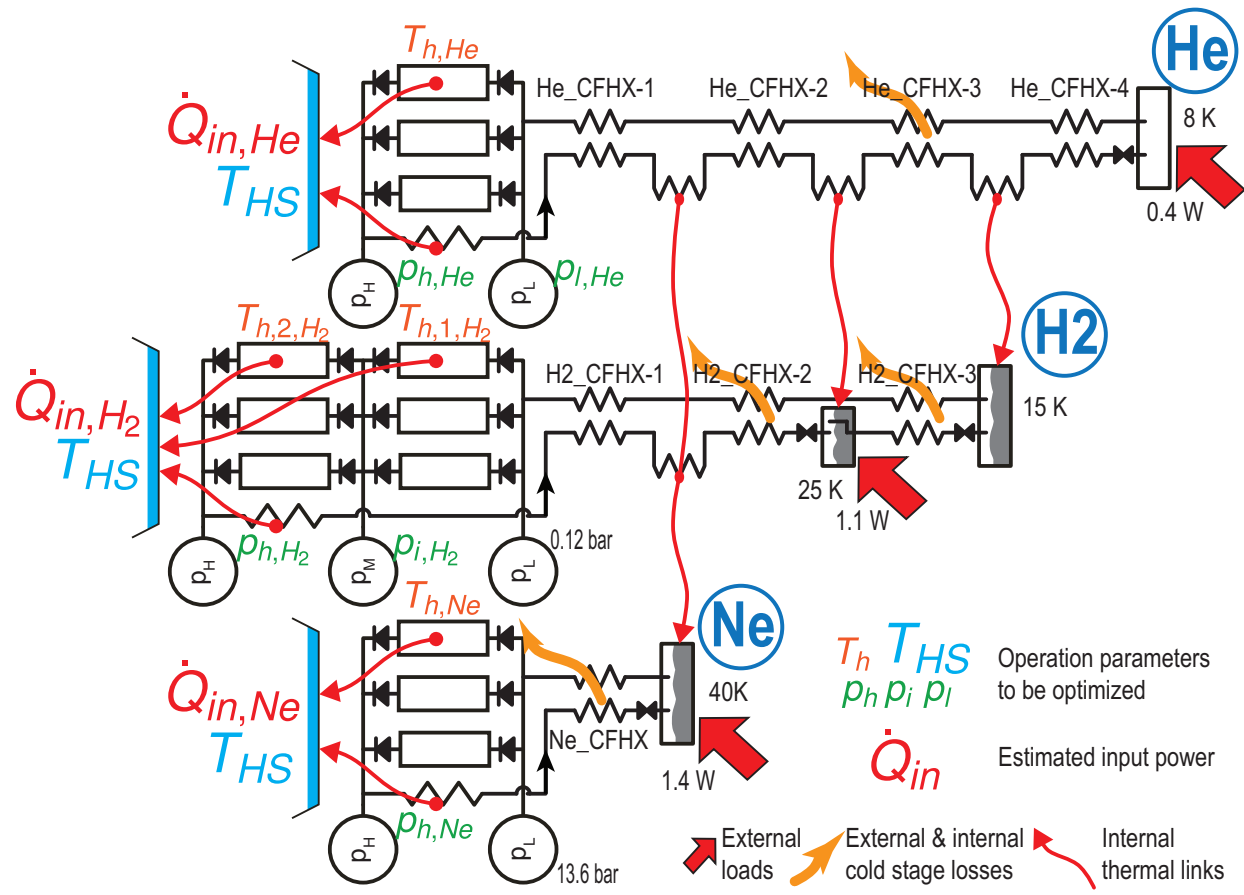

Figure 3.1. Conceptual design of METIS cooler chain. Neon and hydrogen stages precooling the helium stage and together establishing the METIS temperature platforms at $40 \mathrm{~K}, 25 \mathrm{~K}$ and $8 \mathrm{~K}$. 
well accumulated during the previous project in University of Twente [41]. Because the cooling temperature of $8 \mathrm{~K}$ is above the critical temperature of helium, the gas will not liquefy during expansion and a well-designed gas heat exchanger is needed to transfer the heat load to the cold helium gas at the cold tip.

The hydrogen stage provides not only the $25 \mathrm{~K}$ temperature level required both by the METIS instrument but also the $15 \mathrm{sK}$ precooling for the helium stage. Double expansion is used in this stage considering system compactness. A pressure ratio of more than 200 is required. Thus it has to operate with a two-stage compressor.

Finally, a neon-operated stage delivers the required cooling power at $40 \mathrm{~K}$. This stage uses a single-stage compressor and its cooling capacity is split into cooling of the METIS $\mathrm{L} / \mathrm{M}$-detectors and precoolings of the helium and hydrogen stages.

\subsection{Baseline compressor configuration}

The baseline design of the sorption compressor cell remains the concept used in a $4.5 \mathrm{~K}$ helium cooler [40] and 14.5 $\mathrm{K}$ hydrogen cooler in previous studies [41]. As schematically illustrated in Figure 3.2, the cell consists of a cylindrical, thin-wall container that is filled with activated saran carbon pills. Saran carbon is a type of carbon that is well studied and has been used successfully applied in the earlier developments at the University of Twente. The heater is placed at the center of the carbon, and a small gas channel is drilled on the carbon to reduce the axial pressure drop. A filter is used to prevent any carbon particles from flowing into the cold stage causing clogging. A gas inlet tube connects the container to the tubing outside of the cell. Two support spacers made of Inconel alloy hold the container, to maintain a gap between the sorption container and the heat-sink. This narrow gap can act as a gas-gap heat switch (GGHS). The gas pressure in the gap can be varied to yield relatively high or low thermal conduction between the container and the heat sink. In this way, the container can be thermally isolated from the heat sink during the heating phase of the cell, and it can be thermally connected to the heat sink during the cooling phase of the cell. This pressure variation is carried out with the gas-gap actuator,

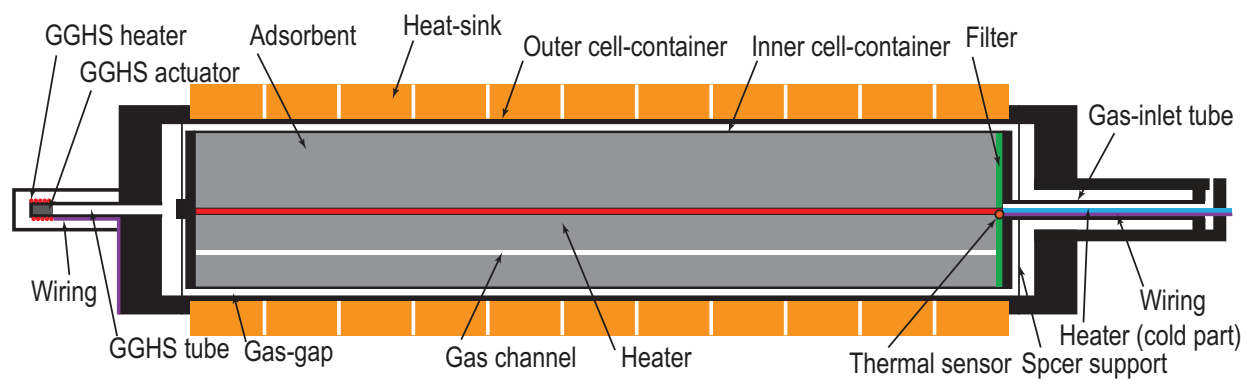

Figure 3.2. Schematic of a sorption compressor cell operating with a gas-gap heat switch (GGHS). 
a small piece of sorber material that is capsuled at the end of a thin-wall tube (GGHS tube) connected to the gas gap and that can be varied in temperature.

The selection of the right adsorbent material and conductive gas for the GGHS depends on the heat-sink temperature, the gas-gap size and the desired ON/OFF conductance ratio that needs to be achieved. Saran carbon and nitrogen were considered first, but the thermal conductance of the "ON" state was limited due to the poor thermal conductivity of the nitrogen. So that this combination required a large number of compressor cells for generating the required mass flow. Using hydrogen significantly reduces the number of compressor cells because hydrogen has a much higher thermal conductivity and thus allows for faster cycling of the cells. However, hydrogen hardly adsorbs onto the saran carbon at around $70 \mathrm{~K}$. Therefore, in the design a small piece of $\mathrm{ZrNi}$ is used that is cycled between $60{ }^{\circ} \mathrm{C}$ and $177^{\circ} \mathrm{C}$. This GGHS actuator is encapsulated at the end of the GGHS tube whose size is well optimized not just to limit the thermal conduction loss to the heat-sink but also to cycle fast enough. It is possible to build an "ON" state of $200 \mathrm{~Pa}$ and an "OFF" state of $0.1 \mathrm{~Pa}$ in the gas gap.

The compressor cells are thermally attached to a cryogenic heat sink, such as a cold platform or a cryogen bath. It will be realized by a dedicated LN2 loop since LN2 is used for cooling the optics of the METIS instrument on E-ELT. The heat-sink temperature is critical to the performance and the size of the system. Generally, the lower the temperature and the higher the pressure, the more gas is adsorbed on the activated carbon. Therefore, as the heat-sink temperature decreases, the efficiency of the sorption compressor increases, while both the size and mass reduce. It is possible to vary the sink temperature in a range of 64 to $74 \mathrm{~K}$ by pumping the $\mathrm{LN} 2$ loop to reduce the pressure on the gas nitrogen exit line (note that the atmospheric pressure on the Cerro Armazones, with an altitude of $3064 \mathrm{~m}$, is about 0.65 bar, leading a boiling temperature of LN2 of $74 \mathrm{~K}$ ).

\subsection{Baseline design}

\subsubsection{Sorption cooler modeling overview}

A thermodynamic quasi-static model based on the analysis described in Chapter 2 is built. By using this model, it is possible to optimize the operating conditions and estimate the performance of the METIS cooler chain on system level.

As shown in Figure 3.3, inputs such as the operating conditions, the cooling temperature, the cooling power, the efficiency of the cooler heat exchangers, compressor-cell geometries, and the relevant material properties are gathered at first. Secondly, the sorption compressor performance normalized to the activated carbon mass that includes the normalized input heat per cycle, $q_{\text {heating }}$ and the normalized fluid mass transferred during a cycle, $\Delta x_{t o t}$, is calculated by the sorption compressor module of the model. The required mass flow rate, $\dot{m}$, is delivered by the JT cold stage (JT-CS) module at the same time. So far the model is static. Combining the static results from the two modules, one can give 


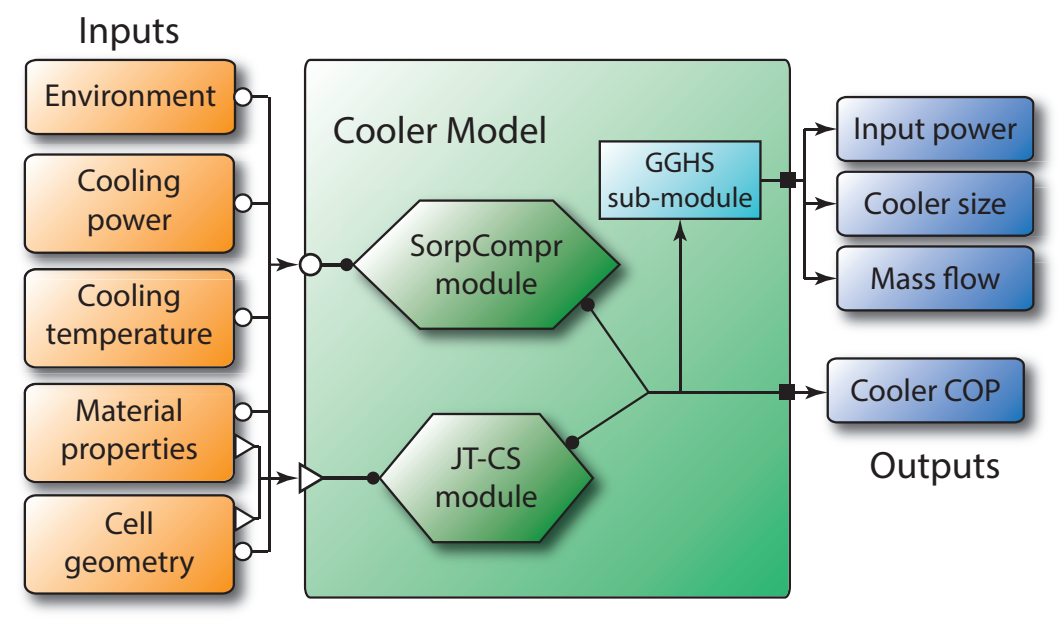

Figure 3.3. Sorption cooler modeling chart.

the total input power into the system, the required mass flow rate and the total coefficient of performance (COP) of the cooler.

In order to have an impression of the cooler size that is more or less dominated by the size of the sorption compressor, it is necessary to introduce the cycle time. To simplify the model, a few assumptions are made:

1. The cylindrical container filled with the activated carbon is considered as a lumped capacity. Therefore, the temperature and the pressure of the carbon are uniform. It is reasonable to assume this if the heating is slow, and the diameter of the cell is not too big. The lumped method will deviate from reality with an increasing cell diameter. The temperature gradient along the radius will influence the sorption compressor performance. Although multi-dimensional dynamic analysis can provide the pressure variation and the temperature distribution, characterizing the system more accurately, the lumped method is more suitable for the investigation on the system level;

2. The cycle time is assumed to be twice of the cooling down time. Usually, the cooling down time is much longer than the heating up time. Therefore, this assumption results in a worst-case estimate of the compressor size;

3. Only the heat transfer through the GGHS and the thermal mass of the cell including the carbon, the container, and the heater, are considered;

4. A constant temperature difference $\Delta T_{l, H S}$ between the heat sink and the sorption container at its minimum operating temperature is assumed.

Following these simplifications, the cooling down time is calculated by considering the sorption cell as a thermal RC system that consists of the thermal resistance via the 
GGHS $\left(R_{G G H S}\right)$ and the thermal capacity of the sorption container $\left(C_{C O N T}\right)$ and the carbon $\left(C_{\text {sor }}\right)$ cycling between a high temperature of $T_{h}$ and a low temperature of $T_{l}$, expressed as,

$$
t_{C D}=\ln \left(\frac{T_{h}-T_{H S}}{\Delta T_{l, H S}}\right) R_{G G H S}\left(C_{C O N T}+C_{s o r}\right)
$$

The cycle time can be then expressed as,

$$
t_{c y c}=2 t_{C D}
$$

and the required carbon mass and the number of compressor cells results as

$$
\begin{gathered}
m_{\text {sor }}=\frac{\dot{m} t_{c y c}}{\Delta x_{\text {tot }}} \\
n_{\text {cell }}=\left\lceil\frac{m_{\text {sor }}}{(1-\alpha) \rho_{\text {sor }, t} V_{\text {CONT }}}\right\rceil
\end{gathered}
$$

in which $\alpha$ is the porosity of the activated carbon. Note that the approximation in this chapter only gives a rough first impression of how many cells may be required for each stages in the METIS cooler chain. In Chapter 5, the cell dimensions will be optimized using a more accurate dynamic model. The cycle times of the sorption compressors will be evaluated more accurately and the design will be updated.

\subsubsection{Optimization of the operating parameters}

The operating parameters include the heat-sink temperature $T_{H S}$ of the LN2 bus, the high temperatures $T_{h}$ of the sorption container, and the unknown pressures, referred as $p_{h}$, $p_{i}$ and $p_{l}$ in Figure 3.1. The input heating power was minimized to find the optimum operating parameters. In this optimization, the optimum temperatures were searched by Newtons method [72] whereas the optimum pressures were found by Fibonacci search method [73].

The JT cold stage is assumed to be ideal, which means perfect CHFXs, precooling HXs and Cold HXs, as well as isenthalpic expansion through the JT restriction. In the optimization, the thermal mass of the container wall, container end caps, and the activated carbon that are temperature dependent were taken into account. The thickness of the container wall was calculated by,

$$
\delta_{w, \text { cont }}=\frac{f_{s}}{f_{w}} \frac{p_{\max } D_{i, c o n t}}{2 \sigma_{y}}
$$

and the thickness of the container end cap was given by,

$$
\delta_{w, \text { cont }}=D_{i, \text { cont }} \sqrt{\frac{f_{s}}{f_{w}} \frac{p_{\max }}{2 \sigma_{y}}}
$$


A safety factor $\left(f_{s}\right)$ of 2.5 and a welding factor $\left(f_{w}\right)$ of 0.7 were chosen. A minimum thickness of $250 \mu \mathrm{m}$ was set for the container wall, and $2.0 \mathrm{~mm}$ for the container end caps considering the technical difficulty. In addition to the porosity of the carbon, a void volume per unit cell length of $2.0 \mathrm{~cm}^{3} / \mathrm{m}$ was assumed for each cell. A temperature difference of $3 \mathrm{~K}$ was assumed between the heat sink and the cell at its minimum operating temperature. As stated before, the cycle time is twice the cooling down time. The temperature difference between the boiling neon and the evaporator outside was assumed to be $0.5 \mathrm{~K}$ and the temperature difference at the hydrogen stage was $0.1 \mathrm{~K}$. There are many additional loss factors that will deteriorate the performance of the cooler. In the system design phase, $25 \%$ margins were added to the requirements of the net cooling power and precooling power to cover of the additional losses in the JT cold stages. Furthermore, the required input power was increased by $10 \%$ to incorporate parasitic terms such as the input power for cycling the GGHS and other losses in the sorption compressor. Table 3.1 lists the assumptions and margins used in the optimization.

The baseline dimensions and operating parameters for a cylindrical sorption compressor cell are presented in Table 3.2, so that a first impression of compressor size and number of cells can be generated for the further detailed design. As mentioned before, the detailed design of the compressor cells will be discussed in Chapter 5.

\section{Helium stage}

Table 3.1. Design margins and assumptions for the operating condition optimization.

\begin{tabular}{|c|c|c|c|}
\hline Parameter & Value & Unit (W) & Description \\
\hline$v_{a d d, L}$ & 2.0 & $\mathrm{~cm}^{3} / \mathrm{m}$ & Additional void volume per unit cell length in each cell. \\
\hline$\Delta T_{l, H S}$ & 3.0 & $\mathrm{~K}$ & $\begin{array}{l}\text { Difference between the heat-sink temperature and the cell mini- } \\
\text { mum operating temperature. }\end{array}$ \\
\hline$t_{c y c}$ & $2 * t_{C D}$ & $\mathrm{~S}$ & Cycle time is taken as twice the duration of the cool-down phase. \\
\hline$\Delta \dot{Q}_{c}$ & 25 & $\%$ & Margin of the cooling power, added to the required cooling powers. \\
\hline$\Delta \dot{Q}_{\text {preC }}$ & 25 & $\%$ & $\begin{array}{l}\text { Margin of the pre-cooling power, added to the required pre-cooling } \\
\text { powers. }\end{array}$ \\
\hline$\Delta \dot{Q}_{i n}$ & 10 & $\%$ & Margin of the input power, applied after all the other margins. \\
\hline
\end{tabular}

Table 3.2. Baseline dimensions and operating parameters for the sorption compressor cell.

\begin{tabular}{|c|c|c|c|}
\hline Parameter & Value & Unit (W) & Description \\
\hline Dia. of the carbon & $10-17.5$ & $\mathrm{~mm}$ & Diameter of the carbon. \\
\hline Len. of the carbon & 50 & $\mathrm{~cm}$ & Length of the carbon (cell). \\
\hline Width of GGHS & 500 & $\mu \mathrm{m}$ & $\begin{array}{l}\text { GGHS width, distance between the container and the } \\
\text { heat-sink. }\end{array}$ \\
\hline Gas of GGHS & Hydrogen & - & - \\
\hline ON pres. of GGHS & 200 & $\mathrm{~Pa}$ & Filling pressure of the GGHS when it is ON. \\
\hline OFF pres. of GGHS & 0.1 & $\mathrm{~Pa}$ & Vacuum pressure of the GGHS when it is OFF. \\
\hline
\end{tabular}


The cooling powers required for the neon and hydrogen stages not only depend on the heat loads from the METIS instrument but also on the pre-cooling power needed by the helium stage. Therefore, the optimization process was started with the helium stage.

The helium stage provides the cooling at the lowest temperature level of $8 \mathrm{~K}$ for the $\mathrm{N}$ band image detector on the METIS instrument. Table 3.3 presents the relevant constraints, operating parameters to be optimized and the corresponding outputs.

The total COP of the helium stage as a function of the low pressure and the high pressure is presented in Figure 3.4, with a heat-sink temperature of $70 \mathrm{~K}$. The same optimization was performed for various heat-sink temperatures in the range of 66-77 $\mathrm{K}$. The results are given in Table 3.4 and Table 3.5. The required input power, as well as the number of sorption cells, decreases significantly with reducing heat-sink temperature. On a pressure-enthalpy (p-h) diagram, the last part of the JT cycle of the helium cold stage is shown in Figure 3.5. The enthalpy is strongly dependent on the temperature, but weakly on the pressure. Consequently the isotherm lines on the $\mathrm{p}-\mathrm{h}$ diagram are almost vertical, and it is hard to create significant enthalpy difference by an isenthalpic expansion, resulting in a low cold-stage efficiency. Also, the helium adsorption amount above $50 \mathrm{~K}$ on saran activated carbon is quite limited resulting in a relatively low sorption compression efficiency. The overall COP of the helium stage is, therefore, expected to be low and a large number of cells are required.

It is clear to see that the high pressure at the JTR inlet (point 8 in Figure 3.5) should not be increased above the inversion point where the Joule-Thomson coefficient $(\partial T / \partial p)_{h}$ is zero. Further increase of the high pressure will only shift the JT expansion line (green dashed line in Figure 3.5) to the right, resulting in a lower enthalpy difference between points 9 and 10, and consequently in less cooling power. Furthermore the optimum high and low pressures do not change much with $T_{H S}$.

The last eight rows of Table 3.4 and Table 3.5 present the effect of the carbon diameter on the required number of cells, the required carbon mass and the cycle time. The diameter of the carbon is used as an input for calculating the $R_{G G H S}$ and $C_{\text {sorp.cont. }}$ in the simplified RC model. Diameters from $10 \mathrm{~mm}$ to $17.5 \mathrm{~mm}$ were investigated. In general, a smaller cell can be cycled faster resulting in less required carbon, but the lower carbon

Table 3.3. Requirements for the helium stage of the METIS cooler.

\begin{tabular}{llclc}
\hline Input parameters & & \multicolumn{3}{c}{ Output parameters } \\
\hline Cooling temp. & $T_{c, \mathrm{He}}$ & $8 \mathrm{~K}$ & Low/High pres. & $p_{h, \mathrm{He}} / p_{l, \mathrm{He}}$ \\
Gross coolign power & $\dot{Q}_{c, \mathrm{He}}$ & $0.5 \mathrm{~W}^{b}$ & High temp. $^{a}$ & $T_{h, \mathrm{He}}$ \\
& $T_{p r e C, 1, \mathrm{He}}$ & $40 \mathrm{~K}$ & Mass flow rate & $\dot{m}_{\mathrm{He}}$ \\
Pre-cooling temp. & $T_{p r e C, 2, \mathrm{He}}$ & $25 \mathrm{~K}$ & Pre-cooling powers & $\dot{Q}_{p r e C, 1 / 2 / 3, \mathrm{He}}$ \\
& $T_{p r e C, 3, \mathrm{He}}$ & $15 \mathrm{~K}$ & Input power & $\dot{Q}_{i n, \mathrm{He}}$ \\
\hline
\end{tabular}

\footnotetext{
${ }^{a}$ Parameters to be optimized.
}

${ }^{b}\left(0.4_{\text {required }}+0.1_{\text {margin }}\right) \mathrm{W}$ 


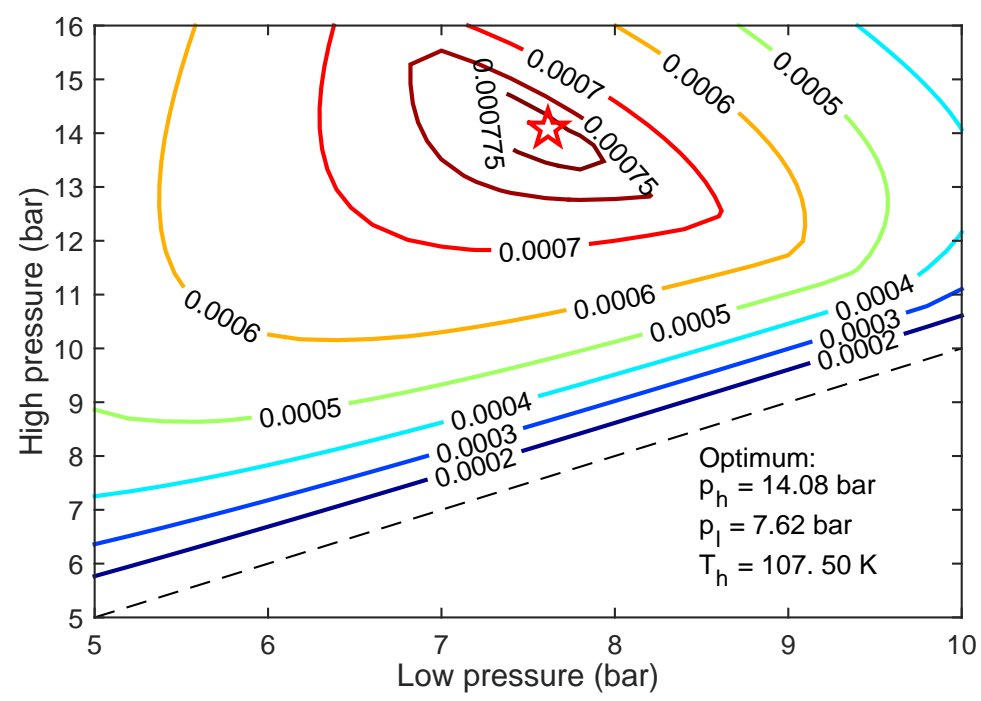

Figure 3.4. Operating parameter optimization for the helium stage of the METIS cooler, $T_{H S}=70 \mathrm{~K}$. The lines indicate constant values of the total COP.

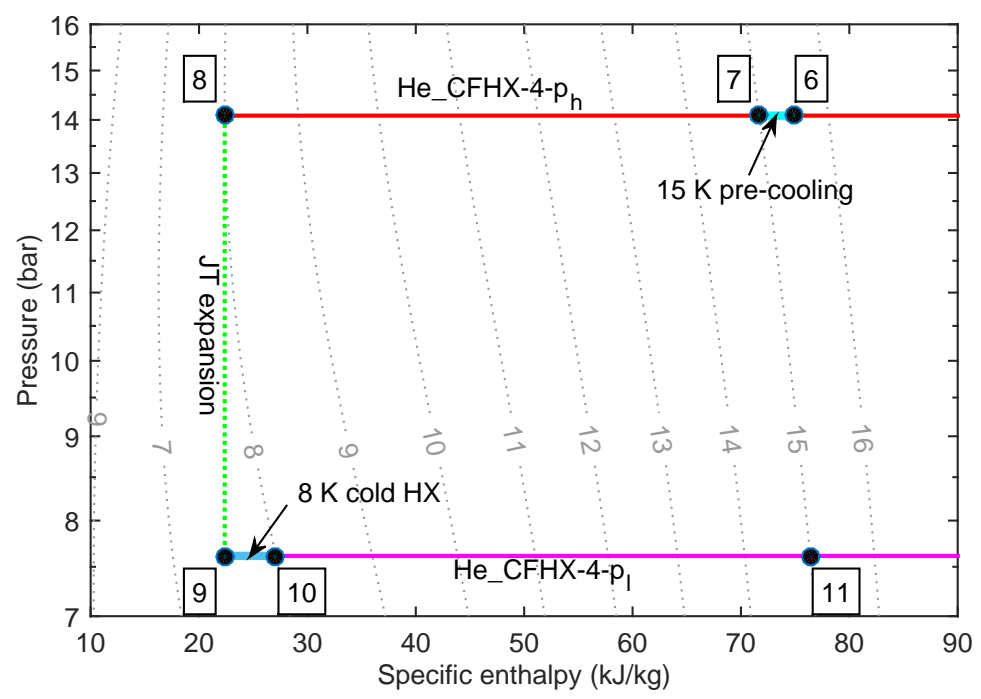

Figure 3.5. Cooling cycle on a p-h diagram for the Helium stage.

mass per cell leads to a larger number of cells. Not that this is a rough estimation which gives an impression of the order of magnitude. The optimization of the cell dimensions is presented in Chapter 5 using a dynamic model that can calculate the cycle time more accurately. 


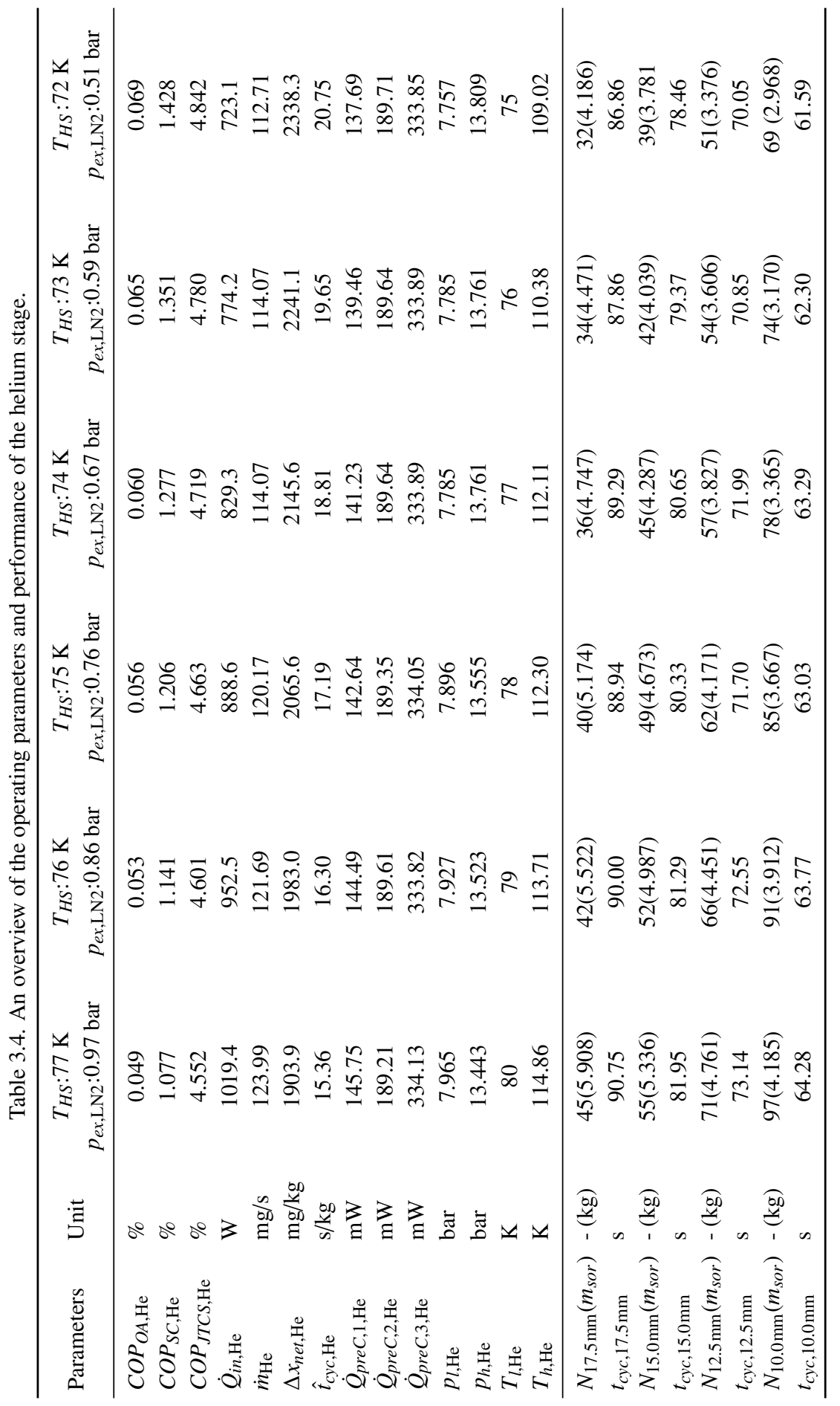




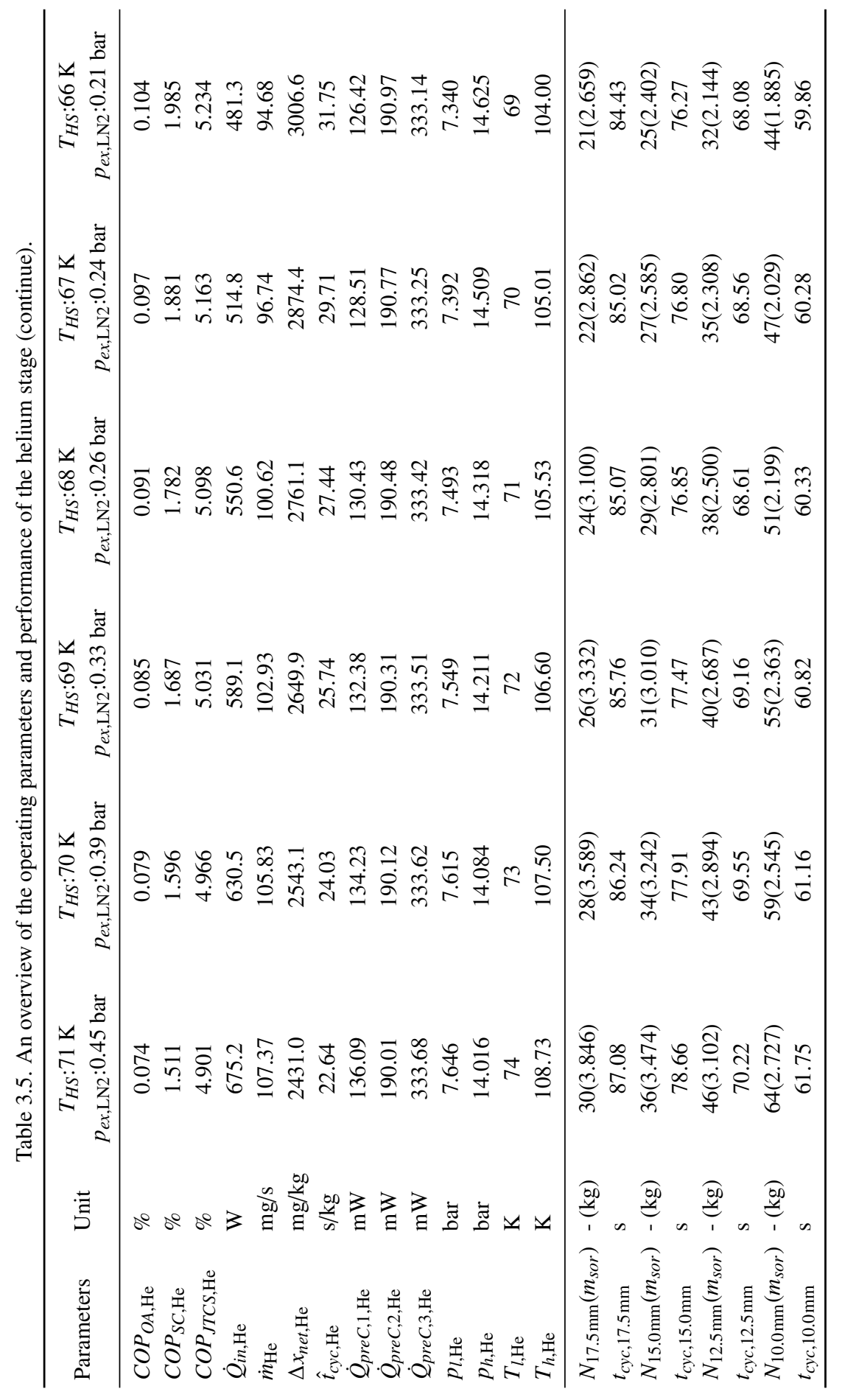




\section{Hydrogen stage}

The hydrogen stage does not only provide the cooling power at $25 \mathrm{~K}$ required by the METIS instrument but also serves as a pre-cooler of the helium stage at $25 \mathrm{~K}$ and $15 \mathrm{~K}$. The relevant constraints, operating parameters to be optimized and the corresponding outputs are listed in Table 3.6. As the hydrogen stage is driven by a two-stage sorption compressor, the intermediate pressure has to be optimized together with the high pressure while individual optimum high temperatures for both stages are searched as well.

With $0.1 \mathrm{~K}$ margin, the boiling-point temperatures of the cooling stages are $24.9 \mathrm{~K}$ and $14.9 \mathrm{~K}$, corresponding to boiling pressures of 3.143 bar and 0.123 bar, respectively. In Figure 3.1 a double expansion of the high-pressure flow is used but other configurations may also be applied. Some alternative concepts are considered in Chapter 4 . The cooling cycle in the double-expansion configuration is plotted on a p-h diagram in Figure 3.6. The high pressure flow expands to 3.143 bar (4-5) and partly liquefies. The liquid evaporates providing cooling at $24.9 \mathrm{~K}(5-6)$. Then the flow leaves the $25 \mathrm{~K}$ cold HX into the third CFHX, where it is partly liquefied in the heat exchange with the cold counterflow low-pressure gas (6-7). In the second expansion, the pressure drops to 0.123 bar, and a temperature of $14.9 \mathrm{~K}$ is reached (7-8). The cold two-phase fluid then vaporizes by taking the cooling load from the precooling $\mathrm{HX}$ of the helium stage (8-9). For a single JT expansion with specific required cooling power, the minimum flow rate is achieved when the fully saturated vapor leaves the evaporator. However in this double expansion configuration with a constant flow rate for both cooling stages, one cannot establish fully saturated vapor at both outlets of the evaporators at the same time with certain cooling loads. Therefore, the flow rate is determined by,

$$
\dot{m}_{\mathrm{H}_{2}}=\max \left(\frac{\dot{Q}_{c, 1, \mathrm{H}_{2}}}{h_{s c, T_{c, 1}}-h_{5}}, \frac{\dot{Q}_{c, 2, \mathrm{H}_{2}}}{h_{s c, T_{c, 2}}-h_{8}}\right)
$$

where subscript "sv" indicates a fully saturated vapor. As a result, there is additional cooling power $\Delta \dot{Q}_{c, j \in\{1,2\}}$ at one of the two temperature levels.

Table 3.6. Requirements for the hydrogen stage of the METIS cooler.

\begin{tabular}{|c|c|c|c|c|}
\hline \multicolumn{3}{|l|}{ Input parameters } & \multicolumn{2}{|l|}{ Output parameters } \\
\hline Cooling temp., 1 & $T_{c, 1, H_{2}}$ & $(25-0.1) \mathrm{K}$ & Intermediate/High pres. ${ }^{a}$ & $p_{\text {im }, \mathrm{H}_{2}} / p_{h, \mathrm{H}_{2}}$ \\
\hline Cooling power, 1 & $\dot{Q}_{c, 1, \mathrm{H}_{2}}$ & $1.38 \mathrm{~W}^{b}+1.25 * \dot{Q}_{\text {preC }, 2, \mathrm{He}}{ }^{c}$ & High temp. ${ }^{a}$ & $T_{h, 1 / 2, \mathrm{H}_{2}}$ \\
\hline Cooling temp., 2 & $T_{c, 2, \mathrm{H}_{2}}$ & $(15-0.1) \mathrm{K}$ & Mass flow rate & $\dot{m}_{\mathrm{H}_{2}}$ \\
\hline Cooling power, 2 & $\dot{Q}_{c, 2, \mathrm{H}_{2}}$ & $1.25 * \dot{Q}_{\text {preC }, 3, \mathrm{He}}{ }^{c}$ & Pre-cooling power & $\dot{Q}_{\text {preC }, \mathrm{H}_{2}}$ \\
\hline Pre-cooling temp & $T_{\text {preC }, \mathrm{H}_{2}}$ & $40 \mathrm{~K}$ & Input power & $\dot{Q}_{i n, \mathrm{H}_{2}}$ \\
\hline
\end{tabular}

${ }^{a}$ Parameter to be optimzied.

${ }^{b}\left(1.1_{\text {required }}+0.28_{\text {margin }}\right) \mathrm{W}$

${ }^{c} \dot{Q}_{\text {preC }, 2, \mathrm{He}}$ and $\dot{Q}_{\text {preC,3,He }}$ are taken from Table 3.4 and Table 3.5 


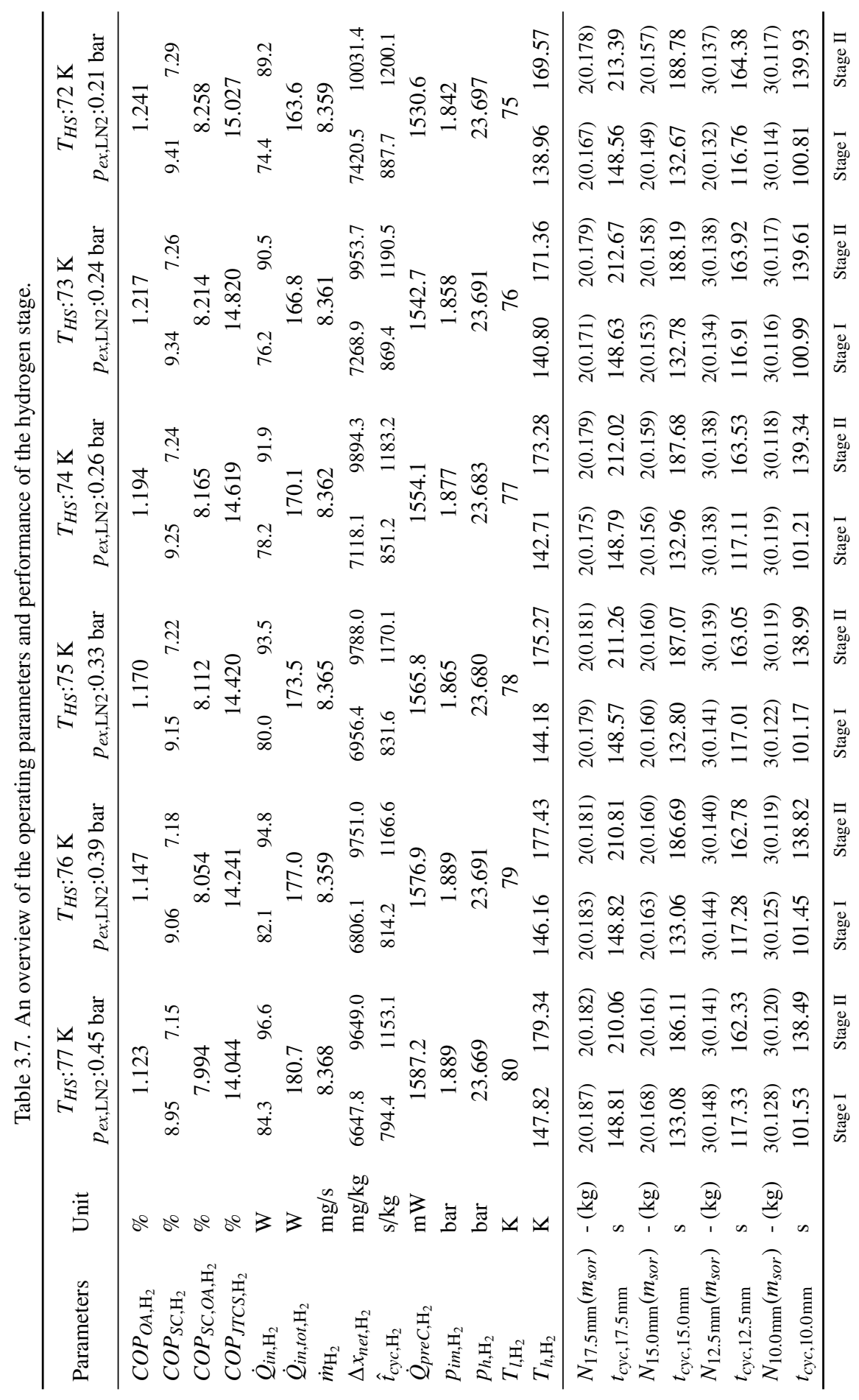




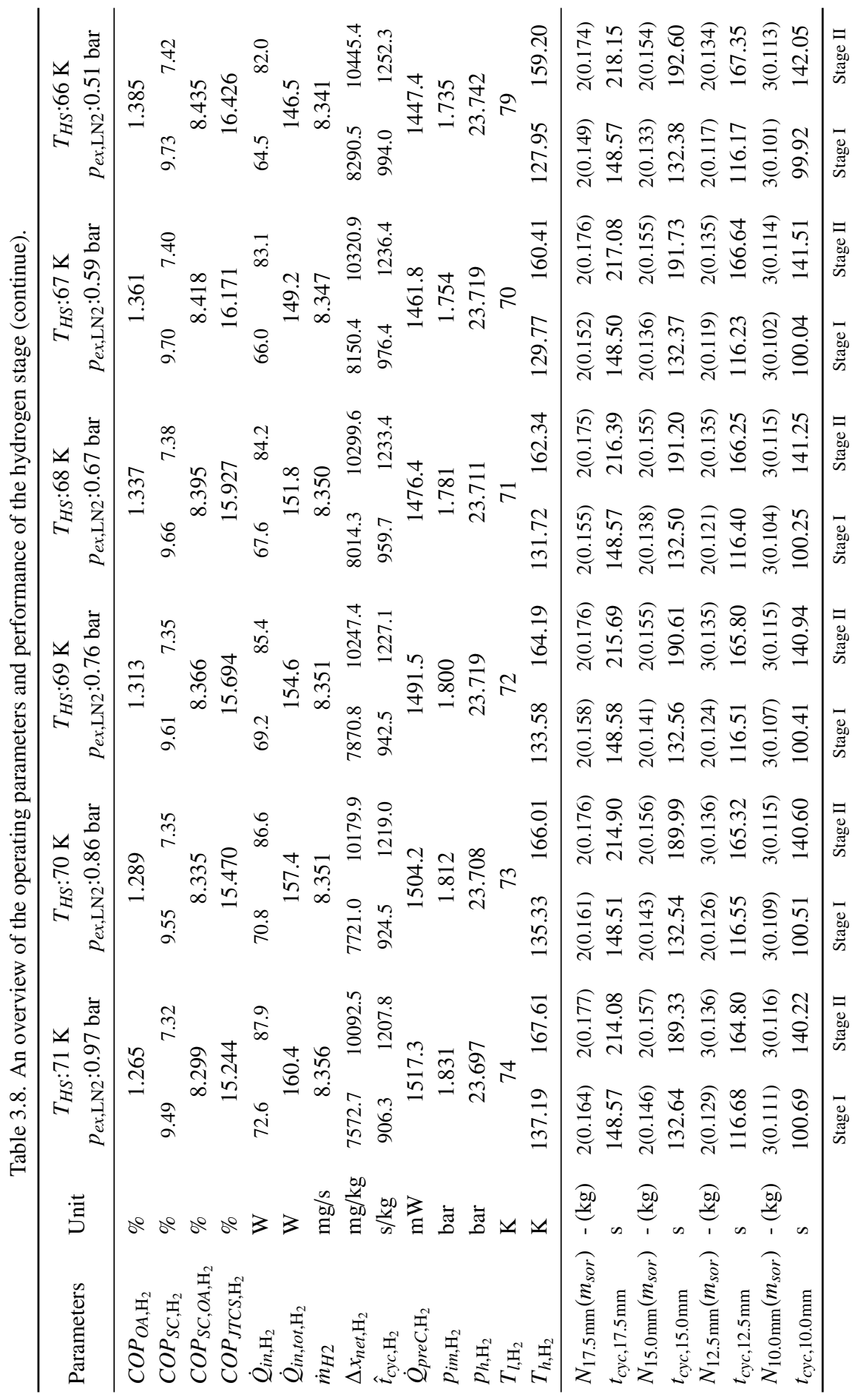




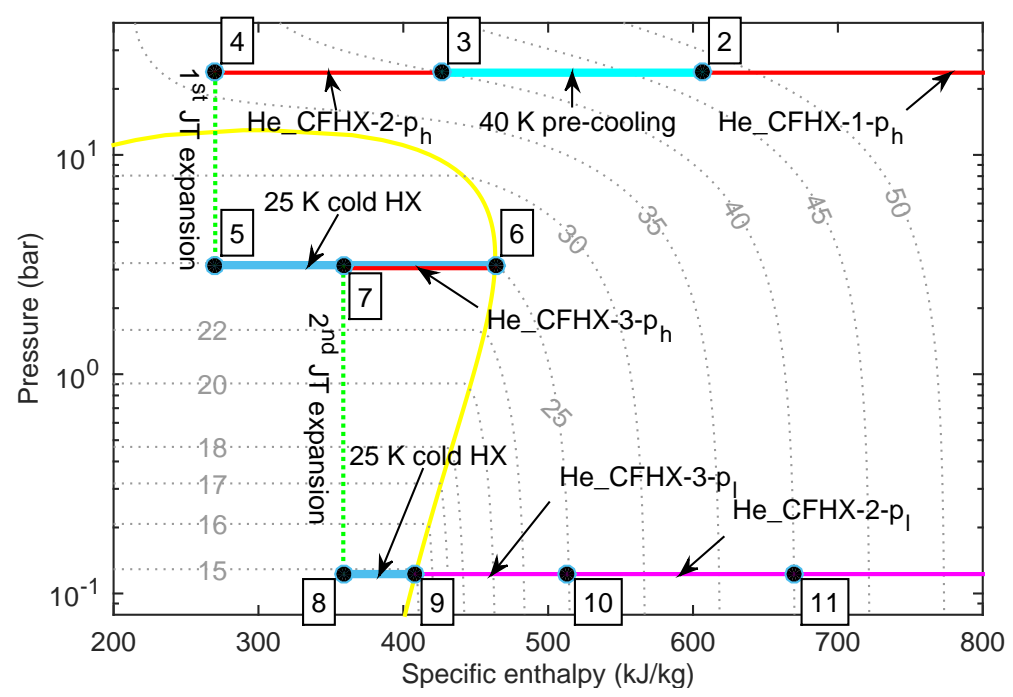

Figure 3.6. Cooling cycle on a p-h diagram for the Hydrogen stage.

The optimization results for the hydrogen stage are given in Table 3.7 and Table 3.8. Since, at around $70 \mathrm{~K}$, the hydrogen adsorption on saran carbon is much higher than that of the helium, the hydrogen compressor efficiency is 6-8 times greater than that of the helium compressor. Consequently, the hydrogen stage needs significantly fewer cells and lower carbon mass. Despite the fact that the required cooling power is three times that of the helium stage, the input power is just a quarter of the helium stage input. Furthermore, similar to the helium stage the input power decreases significantly as the heat-sink temperature decreases. It must be noted that most enthalpy difference for cooling in the hydrogen stage is achieved by precooling at $40 \mathrm{~K}$ (2-3 in Figure 3.6). The pre-cooling power needed is about $1.5 \mathrm{~W}$. This power will be rejected to the evaporator of the neon stage, increasing its cooling capacity requirement by more than a factor of two, as compared to the METIS instrument requirement.

\section{Neon stage}

The neon stage provides the cooling temperature of $40 \mathrm{~K}$ required by the METIS instrument, as well as the precoolings for the hydrogen and the helium stages. The relevant constraints, operating parameters to be optimized and the corresponding outputs are listed in Table 3.9.

The neon stage is a single JT cold stage without any precooling. The cooling temperature is set to be $39.5 \mathrm{~K}$, with a margin of $0.5 \mathrm{~K}$ below the required $40 \mathrm{~K}$. Thus the low pressure is fixed at the corresponding boiling pressure of 13.565 bar which is relatively high to allow the use of a single-stage sorption compressor. Therefore, the optimization is quite straightforward. Table 3.10 and Table 3.11 give an overview of the optimization results of the neon stage. 
Table 3.9. Requirements for the neon stage of the METIS cooler.

\begin{tabular}{lcllc}
\hline Input parameters & & Output parameters & \\
\hline Cooling temp. & $T_{c, \mathrm{Ne}}$ & $(39-0.5) \mathrm{K}$ & High pres. $^{a}$ & $p_{h, \mathrm{Ne}}$ \\
Cooping power & $\dot{Q}_{c, \mathrm{Ne}}$ & $1.75 \mathrm{~W}^{b}+1.25 *\left(\dot{Q}_{\text {preC }, 1, \mathrm{He}}+\dot{Q}_{p r e C, \mathrm{H}_{2}}\right)^{c}$ & High temp. $^{a}$ & $T_{h, \mathrm{Ne}}$ \\
& & & Mass flow rate & $\dot{m}_{\mathrm{Ne}}$ \\
& & Input power & $\dot{Q}_{i n, \mathrm{Ne}}$ \\
\hline
\end{tabular}

${ }^{a}$ Parameter to be optimzied.

$b^{b}\left(1.4_{\text {required }}+0.35_{\text {margin }}\right) \mathrm{W}$

${ }^{c} \dot{Q}_{\text {preC }, 1, \mathrm{He}}$ is taken from Table 3.4 and Table 3.5, while $\dot{Q}_{\text {pre } C, \mathrm{H}_{2}}$ is taken from Table 3.7 and Table 3.8 .

\subsection{Demonstration plan for the METIS cooler}

In order to demonstrate the sorption JT cooler technology for such a large ground application and to validate the design, three demonstration setups were planned based on the baseline design of the METIS cooler chain discussed in this chapter:

1. Full-scale $8 \mathrm{~K}$ helium JT cold stage. This demonstrator (discussed in Chapter 4) is representative of the helium stage in the METIS cooler chain in terms of cooling power, cooling temperature and mass flow, but it is driven by a mechanical compressor. Precooling is supplied by a conventional mechanical cooler. The aim is to validate the cold-stage performance, in particular regarding the 15-8 K CFHX, the $8 \mathrm{~K} \mathrm{CHX}$, and the JT restriction.

2. Scaled helium sorption compressor. The second demo is a scaled version of the helium compressor in the METIS cooler chain, which contains sorption compressor cells of sizes representative for METIS and operates with the same heat-sink temperature as the METIS cooler. However, the number of cells is limited to about one tenth of the METIS helium compressor. The aim is to validate the performance of the helium sorption compressor, in particular regarding COP, cell size, and the dynamic behavior of the sorption compressor. The detailed design of the compressor cells is considered in Chapter 5 whereas the helium compressor demonstrator is discussed in Chapter 6.

3. Scaled $40 \mathrm{~K}$ neon sorption cooler. This is a complete neon-stage demo-cooler establishing about one-fourth of the required cooling power of the neon stage cooler for METIS. The purpose is to demonstrate a full-functional sorption cooler and validate the design of the critical components. This demonstrator is discussed in Chapter 7. 


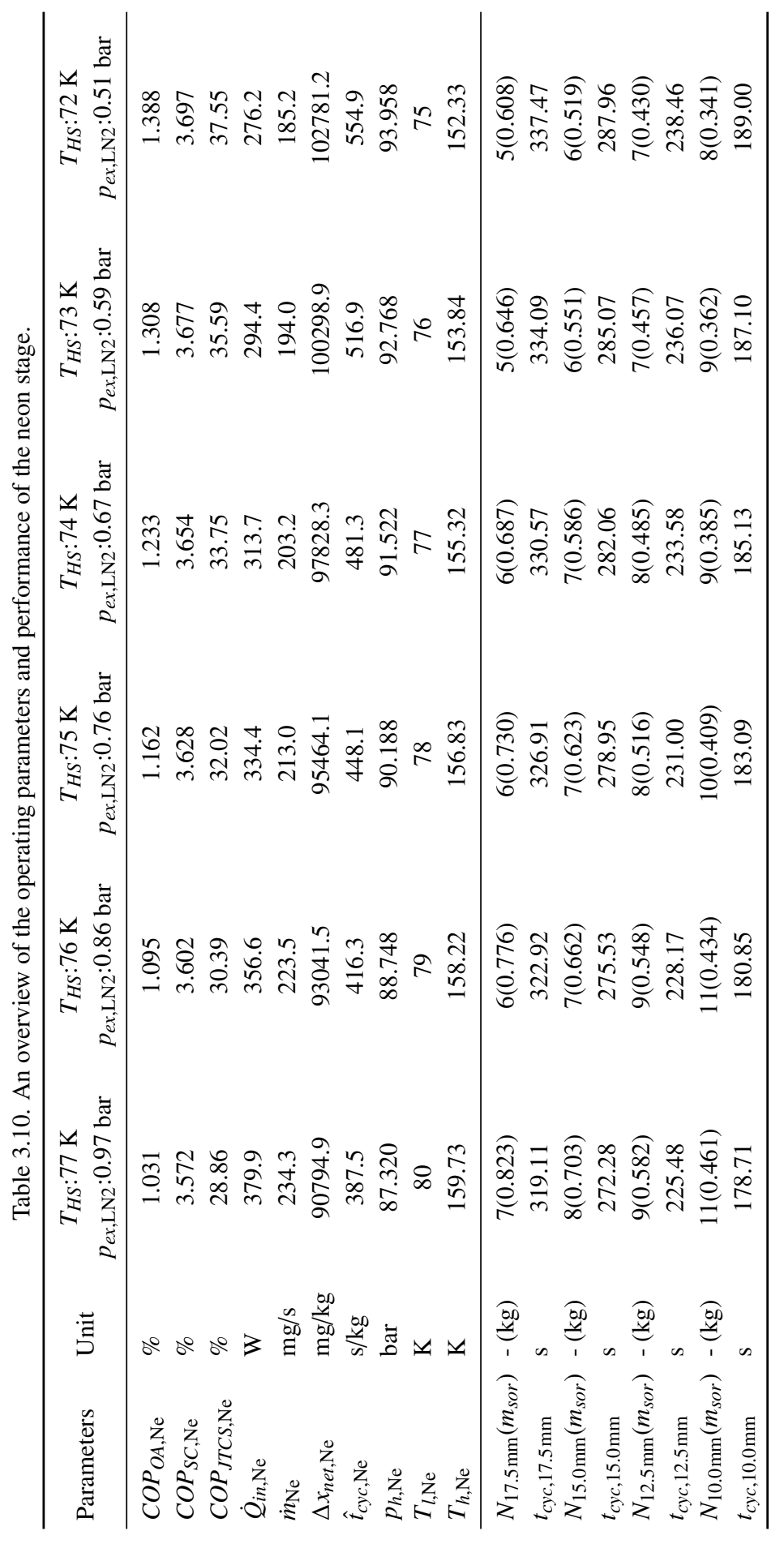




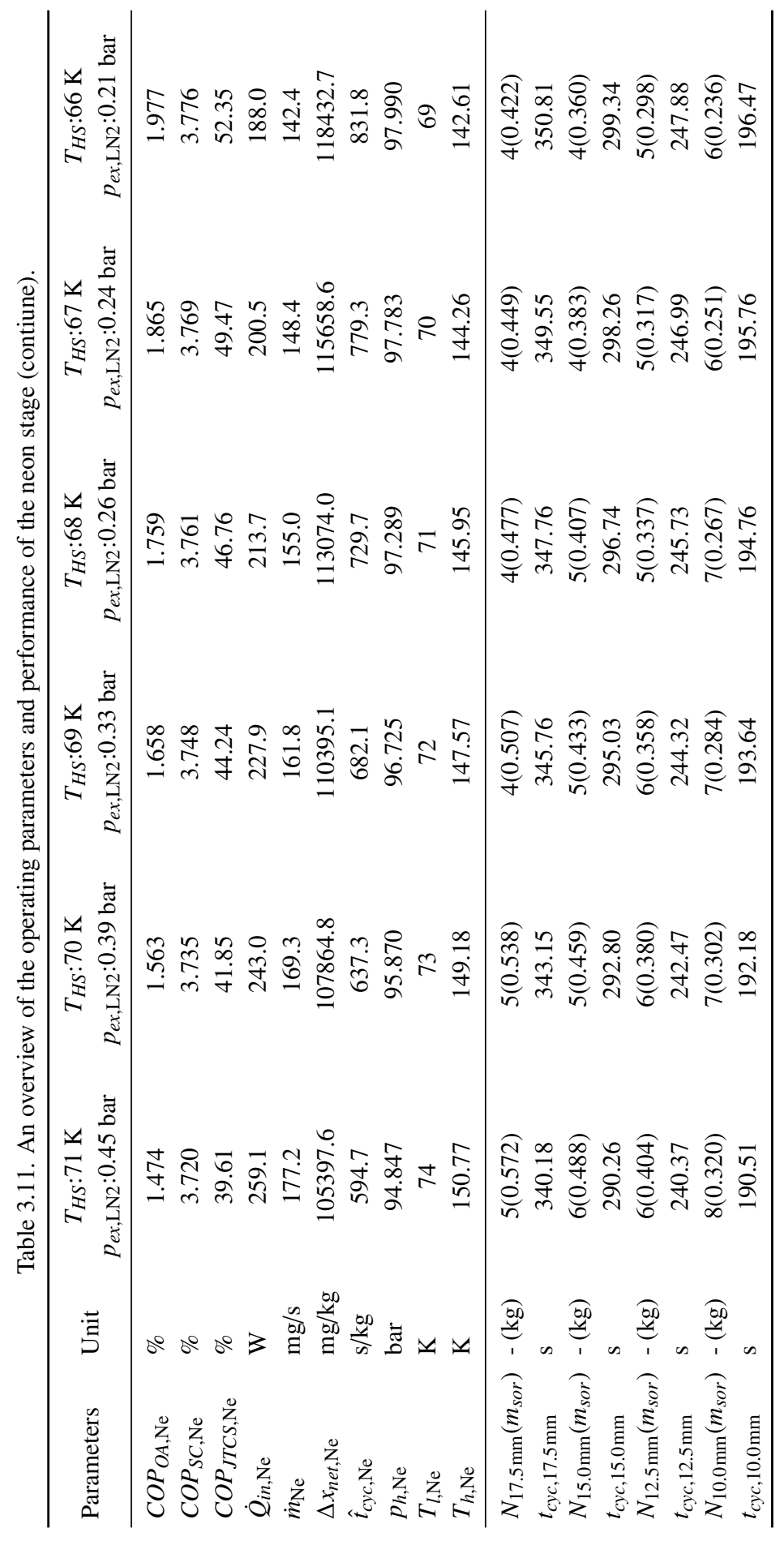




\subsection{Conclusions}

In this chapter, the conceptual design of the METIS cooler chain is presented and primary impressions of configurations, size and performance are given. Figure 3.7 presents a summary of the baseline design. Three stages are thermally linked in parallel and provide cooling at $8 \mathrm{~K}$ (helium), $25 \mathrm{~K}$ (hydrogen) and $40 \mathrm{~K}$ (neon). The heat-sink temperature for the sorption compressors is at $70 \mathrm{~K}$. The helium stage determines the overall input power and size of the system. It operates over a small pressure ratio (only 1.85) but consumes more than $60 \%$ of the total input power $(631 \mathrm{~W})$ and requires 34 cells with a carbon diameter of $1.5 \mathrm{~cm}$ and cell length of $0.50 \mathrm{~m}$ (rough estimation, to be updated in Chapter 5). The hydrogen stage uses a two-stage sorption compressor to compress the hydrogen from 0.12 bar up to 23.7 bar with an input power of $157 \mathrm{~W}$. The neon stage requires a quite high optimum high pressure of almost 96 bar and it requires $243 \mathrm{~W}$ input. In total, the cooler chain requires $1031 \mathrm{~W}$ input at $70 \mathrm{~K}$ (taking into account $25 \%$ margins in (pre)cooling powers and $10 \%$ margin added to the resulting input power). Based on this baseline design, three demonstrators are configured that will be discussed in Chapter 4 to 7 in detail.

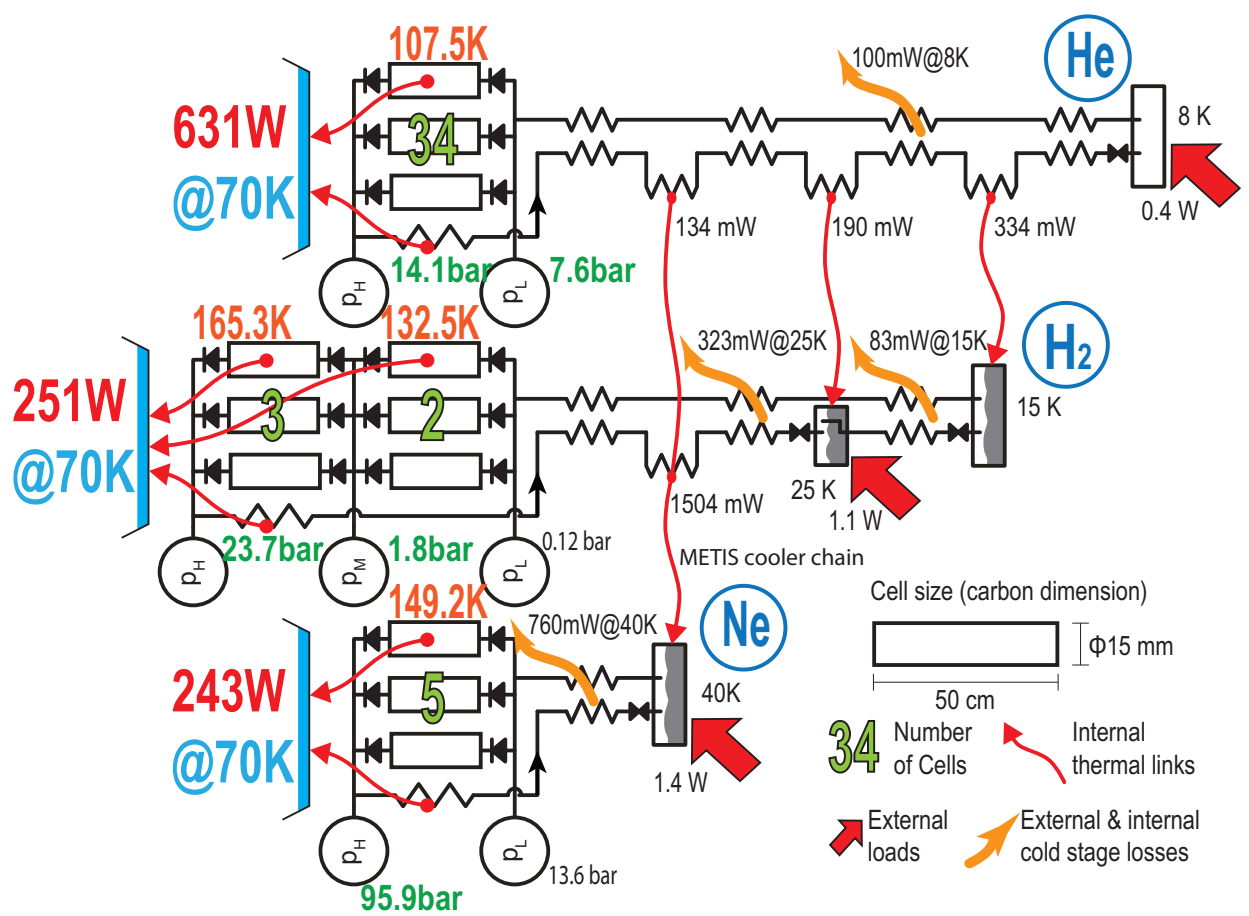

Figure 3.7. Summary of the baseline design of the METIS cooler chain. 



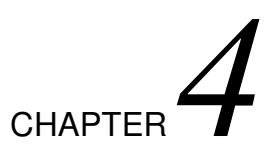

\section{Joule-Thomson Cold Stage Design and Demonstration for the METIS Cooler}

In this chapter, different configurations for the METIS cold stage are considered. The requirements of the counter flow heat exchangers (CFHXs) in the METIS cooler chain are discussed. Then, a numerical model for a tube-in-tube CFHX is introduced. The design of the most critical part of the METIS cold stage is discussed in detail. This most critical part is the lowest temperature section of the helium cold stage. This part comprises the lowest temperature CFHX, the JT restriction, and the heat exchanger to the $8 \mathrm{~K}$ platform. This section was fabricated, tested and demonstrated to comply with the required fullscale METIS performance. The chapter closes with a discussion on these experimental results. 


\subsection{Joule-Thomson cold stage configuration}

In this section, alternative configurations of the METIS cold stage are discussed based on the baseline configuration.

\subsubsection{Parallel configuration vs serial configuration}

In most cases, multi-stage JT coolers are arranged in a parallel configuration $[74,75]$ because of both thermal efficiency and practical complexity. Unlike traditional mechanical compressors that operate at around $300 \mathrm{~K}$, a sorption compressor can operate at cryogenic heat-sink temperature. Thus, the METIS sorption compressors can be heat-sunk at the liquid-nitrogen cooled METIS platform. Or, as a further alternative depicted in Figure 4.1, the helium stage and neon stage can be arranged in series by sinking the helium sorption compressor at $40 \mathrm{~K}$, cooled by the neon stage. Reducing the heat-sink temperature to $40 \mathrm{~K}$ significantly improves the performance and reduces the size of the helium stage. The disadvantage is that it requires more cooling power from the neon stage. This kind of configuration should be selected only if the neon stage is efficient enough so that the gain in the helium stage is more than the cost in the neon stage. However, the COP of the neon stage is very limited, around $1-2 \%$, while the gain factor of the helium stage performance by changing the heat-sink temperature from $70 \mathrm{~K}$ to $40 \mathrm{~K}$ is only about 3.6. Therefore, it is wise to operate the helium stage relatively inefficiently at $70 \mathrm{~K}$ to keep the overall system more efficient.
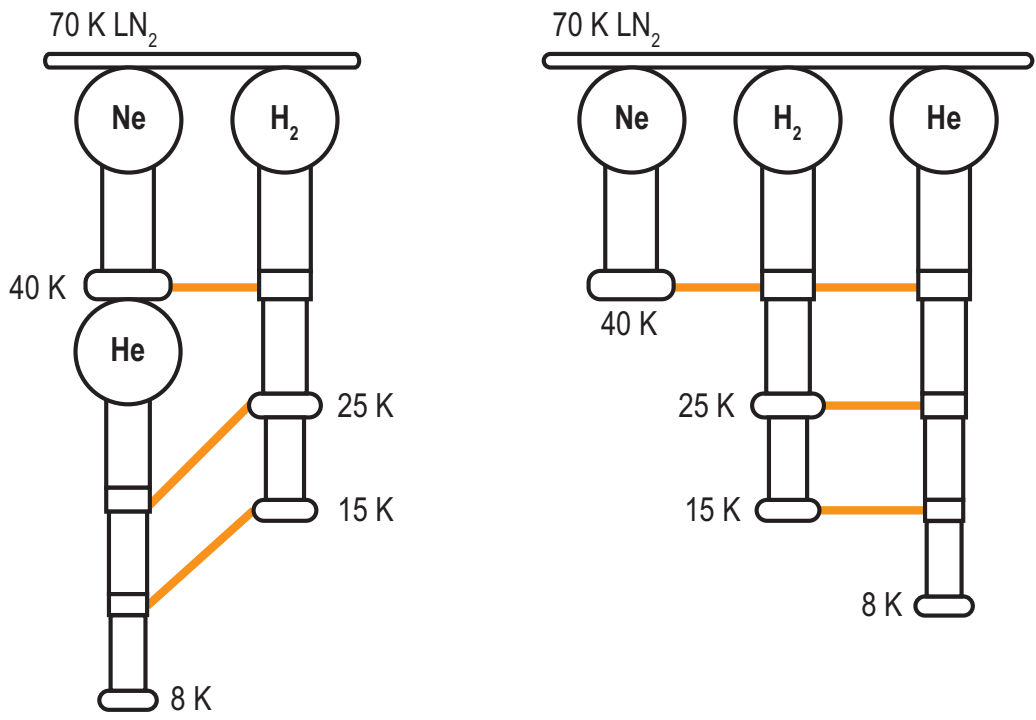

Figure 4.1. Optional (serial) configuration (left) for the METIS cooler chain compared to the baseline (parallel) configuration (right). 


\subsubsection{Alternative hydrogen-stage configurations}

The hydrogen stage in the METIS cooler chain provides cooling at $25 \mathrm{~K}$ and $15 \mathrm{~K}$. There is a variety of possible configurations for the hydrogen stage to achieve these two cooling levels, as shown in Figure 4.2. One straightforward configuration is to arrange two separated stages in parallel (SSP) like Figure 4.2 (a). Two stages operate independently so that optimum high pressure can be found for each stage. However, it requires five CFHXs and two precooling stages, which results a bulky system. In order to reduce the complexity and size of the system, the hydrogen stage can be also realized by a dualpressure Linde-Hampson configuration. The central idea is related to the dual-pressure liquefier of Carl von Linde [76] (see Figure 4.3), in which the high-pressure fluid experiences two successive isenthalpic expansion (also called double expansion). Based on this basic dual-pressure Linde-Hampson configuration (DPLH), there are three variations available for the METIS hydrogen stage as shown in Figure 4.2 (b), (c) and (d), referred as DPLHV1, DPLHV2 and DPLHV3. DPLHV1 adds an additional CFHX between the two evaporators to further cool the saturated liquid at the intermedia pressure to subcooled before it expands via the second JT restriction. In DPLHV2, the flow is separated before the first expansion and the second expansion happens between the high and low pressures rather than between the intermediate and low pressures in DPLHV1. DPLHV1 and DPLHV2 both require a triple-flow CHFX, which is complicated to design and manufacture. Based on DPLHV1, DPLHV3 is proposed for the METIS baseline design that cancels the intermediate pressure line. The advantage is to simplify the CFHX and reduce the cold stage size. However, it requires more input power to compress all the flow from the low to the high pressure. Given the cooling requirement obtained for the hydrogen stage in Chapter 3, the high pressure was optimized to obtain minimum input for all these four configurations and the cold stage performances with the optimum high pressures are listed in Table 4.1.

For the cooling temperatures of 24.9 and $14.9 \mathrm{~K}$, the corresponding vapor pressures

Table 4.1. Cold-stage performance comparison between different configurations.

\begin{tabular}{|c|c|c|c|c|}
\hline \multicolumn{5}{|l|}{ Inputs: } \\
\hline Working fluid & Hydrogen & \multicolumn{2}{|c|}{ Warm end temp., $\mathrm{K}$} & 73 \\
\hline Cooling temp., $\mathrm{K}$ & $24.9 / 14.9$ & \multicolumn{2}{|c|}{ Pre-cooling temp., $\mathrm{K}$} & 40 \\
\hline Cooling power, $\mathrm{W}$ & $1.613 / 0.417$ & \multicolumn{2}{|c|}{ Effectiveness of CFHXs } & $100 \%$ for all \\
\hline \multicolumn{5}{|l|}{ Outputs: } \\
\hline Configuration & SSP & DPLHV1 & DPLHV2 & DPLHV3 \\
\hline$p_{h, o p t}$, bar & $35.98 / 3.21$ & 35.98 & 35.58 & 45.49 \\
\hline$\Delta G_{i n}, \mathrm{~W}$ & $4.49(3.58+0.91)$ & $5.37(4.47+0.90)$ & $5.40(4.47+0.93)$ & 9.78 \\
\hline$\dot{Q}_{\text {preC }} @ 40 \mathrm{~K}, \mathrm{~W}$ & $1.20(1.19+0.01)$ & 1.49 & 1.49 & 1.39 \\
\hline$\dot{m}, \mathrm{mg} / \mathrm{s}$ & $5.01 / 0.92$ & $6.24(5.32+0.92)$ & $6.27(5.31+0.96)$ & 5.57 \\
\hline
\end{tabular}




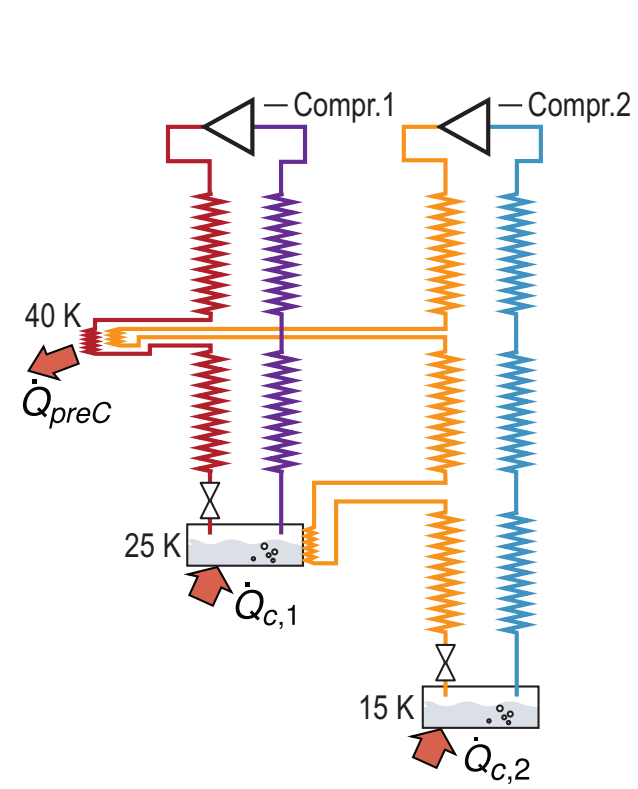

(a) SSP
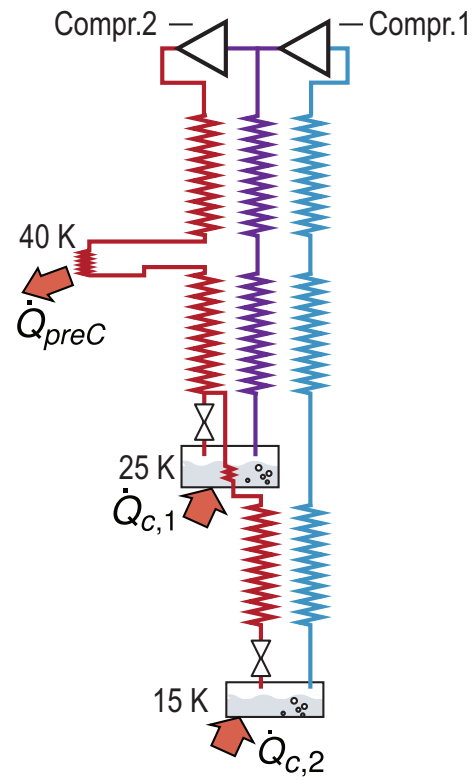

(c) DPLHV2

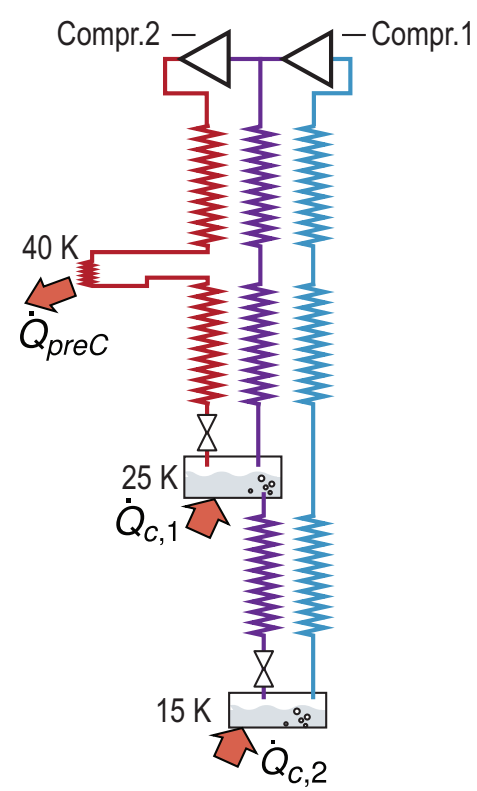

(b) DPLHV1

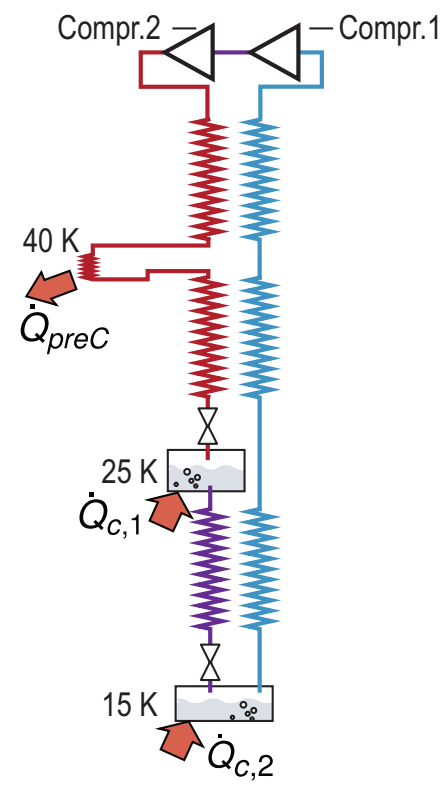

(d) DPLHV3

Figure 4.2. Alternative configurations for the hydrogen stage in the METIS cooler. (a) Separated stages in parallel (SSP); (b), (c) and (d) are three variations of dual-pressure Linde-Hampson configurations (DPLHV1, DPLHV2, DPLHV3). 


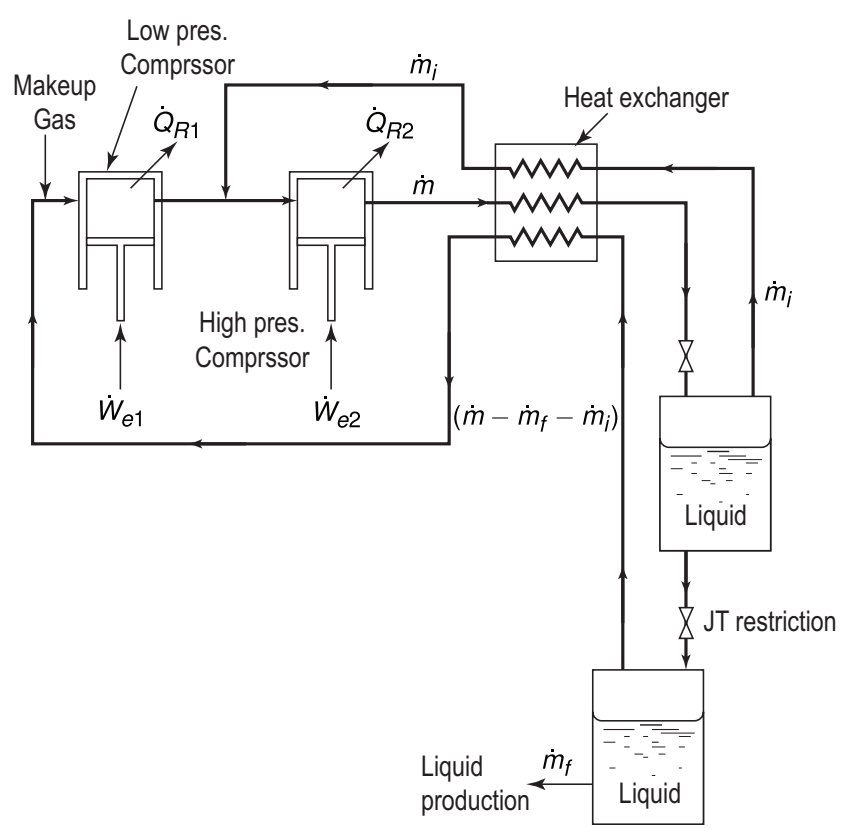

Figure 4.3. Schematics of the dual-pressure liquefier of Carl von Linde [76].

are 3.143 and 0.123 bar. In the SSP configuration, the optimum high pressure can be obtained for both stages. It, therefore, results in highest efficiency (lowest input) and lowest flow rate compared to the other configurations. In three variations of dual-pressure Linde-Hampson configurations, the high pressure for the $14.9 \mathrm{~K}$ cooling is determined either by the evaporation pressure of the $24.9 \mathrm{~K}$ cooling (for DPLHV1 and DPLHV3) or by the high pressure of the $24.9 \mathrm{~K}$ cooling (for DPLHV2). The DPLHV1 has the same optimum high pressure as the $24.9 \mathrm{~K}$ stage in the SSP because the evaporation pressure of $24.9 \mathrm{~K}$ cooling ( $3.143 \mathrm{bar}$, the intermedia pressure) is very close to the optimum high pressure (3.21 bar) for the $14.9 \mathrm{~K}$ cooling. In its evaporator, $85 \%$ of the fluid flows returns to the warm end while the rest of the fluid continues to the next JT restriction via CFHX3 to produce cooling at $14.9 \mathrm{~K}$. The performance of DPLHV2 is very similar to that of DPLHV1 since the flow rate for the $14.9 \mathrm{~K}$ cooling stage is much smaller than that for the $24.9 \mathrm{~K}$ stage. DPLHV3 is the least efficient configuration. It also requires higher high pressure that will consequently be even less competitive with respect to the efficiency once the compressor is considered.

The performance of the SSP, DPLHV1 and DPLHV2 are close, and clearly superior to the DPLHV3 that is considered in the baseline design in Chapter 3. Therefore, we recommend investigating these three alternative configurations for the further design. To decide which configuration can best be used, the sorption compressor, the sizes and complexity of the cold stage and the manufacturing feasibility have to be considered and compared in more detail. 


\subsection{Required effectiveness of the CFHXs in METIS cool- er chain (baseline)}

A JT cold stage for cryogenic cooling usually consists of one or more CFHXs, pre-coolers, JT restrictions, and evaporators. There are eight CFHXs in the baseline design of METIS cooler, operating from $70 \mathrm{~K}$ to $40 \mathrm{~K}, 40 \mathrm{~K}$ to $25 \mathrm{~K}, 25 \mathrm{~K}$ to $15 \mathrm{~K}$ and $15 \mathrm{~K}$ to $8 \mathrm{~K}$, respectively. At these low temperature ranges, any tiny decrease in CFHX effectiveness (as defined in Sec 2.1.2) will cause a considerable increase in required mass-flow rate at specific cooling power, increasing the input power and the size of the compressor [44], as illustrated in Figure 4.4.

However, a high effectiveness of a CFHX requires a large heat transfer area that results in a large size and pressure drop. Therefore, a trade-off between the performance and size has to be made. At the same time, the eight CFHXs in the METIS cooler do not all have the same effect on the overall efficiency of the entire system. One of the CFHXs can be very critical to the cooler performance, and has a strict effectiveness requirement, whereas another CFHX can be less important and may have a relatively low effectiveness.

An analysis of the total input power sensitivity with respect to the effectiveness of the CFHXs is carried out, and the results are presented in Table 4.2. In this analysis, the minimum required effectiveness for each CFHX was determined corresponding to an input increase of less than $1.5 \%$ of the ideal case while all other CFHXs were assumed to be perfect. These minima were used to define recommended values for all CFHXs. With this set of recommended effectiveness values, the input increase compared to the

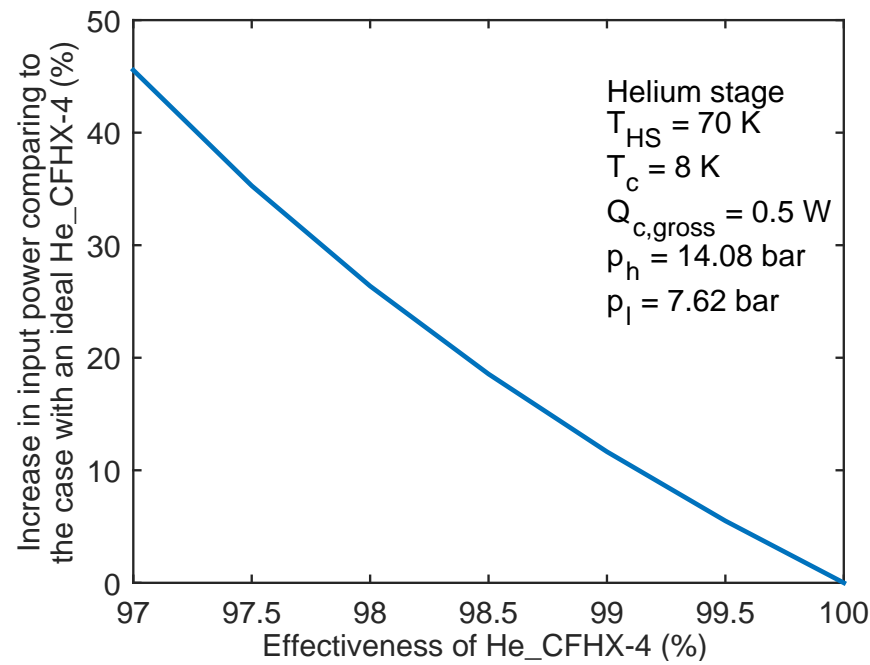

Figure 4.4. The increase in the input power required by the helium stage in the METIS cooler as the effectiveness of the coldest helium CFHX (He_CFHX-4 in Figure 3.1) deviating from the ideal case (in which $E_{C F H X}=100 \%$ ). 
Table 4.2. Required effectiveness of CFHXs (CFHX reference as indicates in Table 3.1).

\begin{tabular}{lccccc}
\hline Ref. CFHX & Min. Eff. & Recom. Eff. & Ref. CFHX & Min. Eff. & Recom. Eff. \\
\hline Ne_CFHX & $\geqslant 95.7 \%$ & $98 \%$ & He_CFHX-1 & $\geqslant 98.6 \%$ & $99 \%$ \\
H2_CFHX-1 & $\geqslant 91.7 \%$ & $98 \%$ & He_CFHX-2 & $\geqslant 98.4 \%$ & $99 \%$ \\
H2_CFHX-2 & $\geqslant 98.4 \%$ & $99 \%$ & He_CFHX-3 & $\geqslant 99.7 \%$ & $99.7 \%$ \\
H2_CFHX-3 & $\geqslant 97.5 \%$ & $98 \%$ & He_CFHX-4 & $\geqslant 99.8 \%$ & $99.8 \%$ \\
\hline
\end{tabular}

baseline is $12.7 \%$ for the neon stage, $8.7 \%$ for the hydrogen stage, $2.1 \%$ for the helium stage, and about $5.7 \%$ for the entire cooler. In other words, with the recommended CFHX effectiveness, the baseline input power will give $11.3 \%$ less cooling power for the neon stage, $8.0 \%$ less for the hydrogen stage and $2.1 \%$ for the helium stage compared to the ideal cooler with perfect CFHXs. Furthermore, Table 4.2 shows that (as may be expected) the coldest helium-stage CFHX (He_CFHX-4) is most critical.

\subsection{CFHX design}

\subsubsection{Select the CFHX type}

There is a variety of CFHX types, generally classified as tubular, plate and fin, or perforated plate [44]. Tubular heat exchangers use metal tubes that are strong, robust, and can be easily fabricated into complex assemblies. Its simple geometry of the flow channel also makes it economically competitive for small-scale applications and its design is relative easy and accurate. Although, usually, it is not as compact as fin or perforated

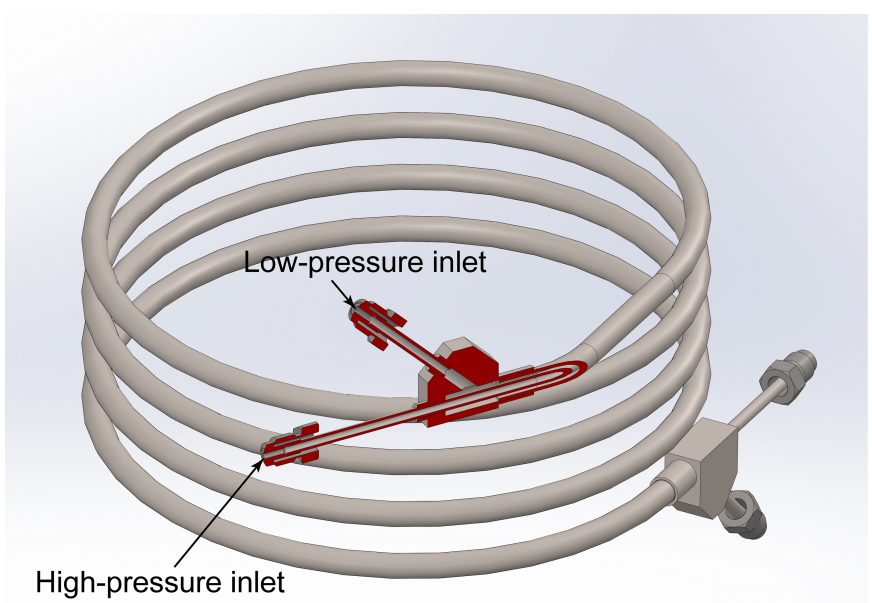

Figure 4.5. CAD view of the assembly of a tube-in-tube CFHX. The splitter at the high-pressure inlet is presented in a cross-sectional view. 
plate heat exchangers. The simplest form of a tubular heat exchanger is the tube-in-tube or double-pipe CFHX. As shown in Figure 4.5, a tube-in-tube CFHX has two concentric tubes creating the inner and outer channels for the warm and cold fluids flow in opposite directions. Normally the high-pressure flow is contained in the inner tube to minimize the required tube wall thickness. Splitters are used to separate the two flows at the inlet and outlet. Tube-in-tube CFHXs in the JT cooler are usually quite long and wound into a helical coil shape to realize a compact size as shown in Figure 4.5.

Tube-in-tube CFHXs are widely used in recuperative cryogenic coolers, such as dilution refrigerators, Linde-Hampson liquefiers, as well as in Joule-Thomson coolers. In this research, we chose the tube-in-tube heat exchanger as the CFHX type for the METIS cooler. The further modeling and design discussed in this section are based on this configuration.

\subsubsection{CFHX Modelling}

In order to design efficient tube-in-tube CFHXs for the METIS cooler, a static 1-dimensional model was built to model the fluid dynamics and heat transfer in the CFHX. In this model, only longitudinal gradients in temperature and pressure are considered whereas the temperatures and pressures in the radial direction for each flow and tube are assumed to be uniform. As usually is the case, the high-pressure flow is in the inner tube while the low-pressure fluid flows through the annular channel between the tubes. In the stationary case, the energy balances for the high-pressure flow, inner tube, low-pressure flow and outer tube can, respectively, be written as,

$$
\begin{gathered}
\dot{m} \frac{\partial h_{h p}}{\partial x}+\pi D_{i, i t} U_{h p}\left(T_{h p}-T_{i t}\right)=0 \\
\frac{\partial}{\partial x}\left(A_{c, i t} k_{i t} \frac{\partial T_{i t}}{\partial x}\right)=-\pi D_{i, i t} U_{h p}\left(T_{h p}-T_{i t}\right)+\pi D_{o, i t} U_{i, l p}\left(T_{i t}-T_{l p}\right) \\
\dot{m} \frac{\partial h_{l p}}{\partial x}+\pi D_{o, i t} U_{i, l p}\left(T_{i t}-T_{l p}\right)+\pi D_{i, o t} U_{o, l p}\left(T_{o t}-T_{l p}\right)=0 \\
\frac{\partial}{\partial x}\left(A_{c, o t} k_{o t} \frac{\partial T_{o t}}{\partial x}\right)=\pi D_{i, o t} U_{o, l p}\left(T_{o t}-T_{l p}\right)+\pi D_{o, o t} \varepsilon_{o, o t} \sigma F\left(T_{o t}{ }^{4}-T_{a m b}{ }^{4}\right)
\end{gathered}
$$

where $\dot{m}$ is the mass flow rate of the flow, $h$ is the specific enthalpy of the fluid, $x$ is the longitudinal coordinate, $D$ is the diameter of the tube, $U$ is the convective heat transfer coefficient, $A_{c}$ is the cross-sectional area of the tube wall, $k$ is the thermal conductivity of the tube, $\epsilon$ is the emissivity of the tube surface, $\sigma$ is the Stefan-Boltzmann constant, $F$ is the view factor from the outer surface of the outer tube to the environment and $T_{a m b}$ is the environment temperature. And the subscripts hp and lp indicate high-pressure and low-pressure flows, $i$ and o represent inner and outer surfaces of the tubes, and it and ot refer to inner tube and outer tube.

As schematically shown in Figure 4.6, the model is 1-dimensional, and the tube-intube CFHX is divided into finite elements in the longitudinal direction. The temperatures 


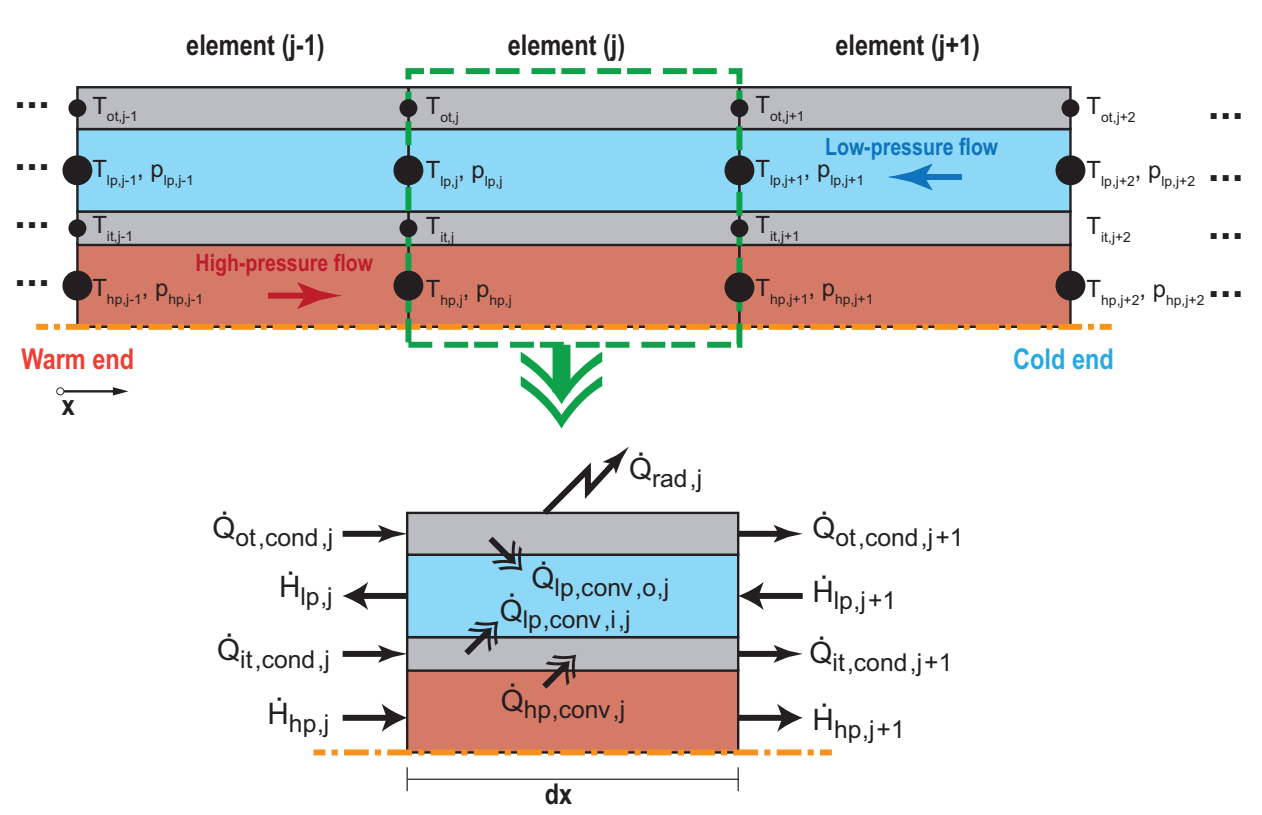

Figure 4.6. Schematic of the CFHX model. Top: CFHX divided into finite elements; temperatures and pressures on the boundaries are of interest. Bottom: heat transfers and enthalpy flows in a finite element.

and pressures at the element boundaries are of the interest, and they are assumed to be linearly distributed in each element. There is no radial temperature or pressure gradient in the flows or tubes. The convection heat transfers between the flows and tube walls are calculated according to the temperature difference between the fluid and solid. The conduction heat transfers through the tubes in the longitude direction are included. Furthermore the radiation heat transfer from the environment to the out surface of the out tube is also calculated, which will affect the temperature profile in the CFHX quite significantly (especially in the range of 70-300 K). We assume the environment (usually the radiation shield) has much larger area than the CFHX, and the view factor is 1 . With this assumption, the model estimates the radiation losses as a worst case.

To obtain high effectiveness ( $>99 \%$ ), tube-in-tube CFHXs usually require quite long length for adequate heat-transfer area, resulting in an increased pressure drop in the flow. It is necessary to evaluate this pressure drop. The cooling temperature of a JT cold stage is determined by the evaporating pressure, i.e. the pressure at the low-pressure inlet of the last CFHX (the coldest one that connects to the evaporator). To establish a certain evaporating pressure, the pressure drop along the low-pressure channel will reduce the required intake pressure of the compressor resulting in a higher compressor input power. Furthermore, the pressure drop at the high-pressure side directly reduces the enthalpy difference at the cold tip resulting in less gross cooling power. The pressure drops in the 
flows are calculated by the Darcy-Weisbach equation,

$$
\begin{aligned}
-\frac{\partial p_{h p}}{\partial x} & =\frac{f_{D, h p}}{D_{h, h p}} \frac{\dot{m}^{2}}{2 \rho_{h p} A_{c, h p}{ }^{2}} \\
\frac{\partial p_{l p}}{\partial x} & =\frac{f_{D, l p}}{D_{h, l p}} \frac{\dot{m}^{2}}{2 \rho_{l p} A_{c, l p}{ }^{2}}
\end{aligned}
$$

To solve the equation system 4.1-4.6, the boundary conditions are defined by the inlet temperatures and pressures of the flows,

$$
\begin{array}{ll}
T_{h p, x=0}=T_{h p, \text { inlet }} & T_{l p, x=L}=T_{l p, \text { inlet }} \\
p_{h p, x=0}=p_{\text {hp,inlet }} & p_{l p, x=L}=p_{l p, \text { inlet }}
\end{array}
$$

and temperatures of the tubes at the warm end,

$$
T_{i t, x=0}=\left.\frac{T_{h p}+T_{l p}}{2}\right|_{x=0}, T_{o t, x=0}=T_{l p, x=0}
$$

We assume that, at the warm end, the inner tube temperature equals the average value of the flow temperatures, and the outer tube temperature is the same as the low-pressure flow. This approximation is reasonable since the temperatures of the high-pressure and low-pressure flows are very close at the warm end for a high-efficiency CFHX (a JT cold stage has the same working fluid and flow rate at both sides).

As an example, Figure 4.7 shows the simulation results for the CFHX in the neon stage of the METIS cooler. Here, the CFHX is $3 \mathrm{~m}$ long, made of $1 / 4 * 0.049$ inch and $1 / 8 * 0.028$ inch tubes. The high-pressure flow enters the CFHX at $73 \mathrm{~K}$ and $95.87 \mathrm{bar}$, whereas the low-pressure flow enters the CFHX at $40 \mathrm{~K}$ and 13.57 bar. The CFHX operates between $73 \mathrm{~K}$ and $40 \mathrm{~K}$ with a flow rate of $169.3 \mathrm{mg} / \mathrm{s}$. The simulation shows that this CFHX has an effectiveness $E_{C F H X}$ of up to $99.85 \%$ and the pressure drops are rather limited (5.20 mbar at the high-pressure side and $33.32 \mathrm{mbar}$ at low-pressure side). At lower temperatures, as the two-phase regime is approached, the enthalpy of the gas becomes more and more pressure dependent. It decreases with pressure. For that reason, at the cold end a temperature difference occurs between the two flows and the temperature gradient along the CFHX is specifically present at the low-temperature end. Since, at the warm end, the temperatures of both flows are very close the convective heat flow from the high-pressure to the low-pressure line is very small, comparable to the radiative heat load at the outer tube at that location. At the cold end the convective heat transfer rate is much larger than the radiative heat load. The total radiation loss of the entire CFHX is very small: $1.14 \mathrm{~mW}$. Furthermore thanks to the long tube made of low-conductivity stainless steel, the longitudinal conduction heat transfer through the tubes is also very tiny, less than $0.01 \mathrm{~mW}$ at the warm end and about $10 \mathrm{~mW}$ at the cold end. 


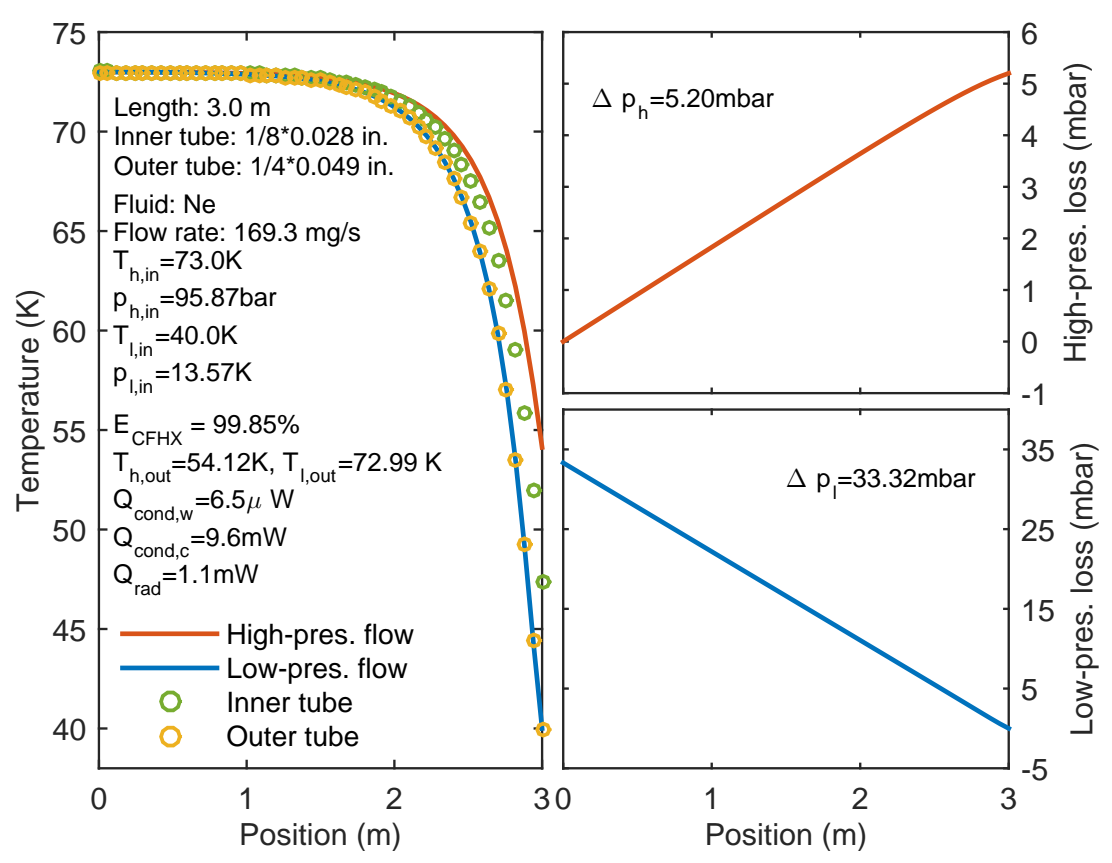

Figure 4.7. An example of simulation results of the CFHX model. The model calculates the temperatures and pressures for the tube-in-tube CFHX in the neon stage of the METIS cooler. Conductive heat flows are given at warm and cold ends of the CFHX, as well as the total radiative heat load.

\subsection{Full scale, $8 \mathrm{~K}$ helium Joule-Thomson cold stage}

\subsubsection{An outlook of the experiment setup}

The purpose of this setup is to validate the design of the most critical components in the JT cold stage: He_CFHX-4 (the most important CFHX in the METIS cooler chain), the $8 \mathrm{~K}$ cold heat exchanger, the JT restriction. Therefore, the experimental setup will focus on the $15-8 \mathrm{~K}$ part of the helium stage in the METIS cooler chain.

Figure 4.8 presents a schematic overview of the full scale, $8 \mathrm{~K}$ helium JT cold stage experimental setup. The 15-8 K part was built upon an existing, powerful precooling subsystem. The precooling subsystem has two tube-in-tube CFHXs (CFHX_300-70 and CFHX_70-15) as shown in Figure 4.8 in series that precools the compressed high-pressure flow down to $15 \mathrm{~K}$. A powerful two-stage GM cooler, Sumitomo RDK-408D2, simulates the neon and hydrogen stages in the METIS cooler chain to supply precooling at intermediate temperatures (70 $\mathrm{K}$ and $15 \mathrm{~K}$ in this setup). CFHX_300-70 and CFHX_70-15 were wound into helical coils that surround the GM cooler. At the cold ends of CFHX_300-70 and CFHX_70-15, the high-pressure lines are split from the tube-in-tube structures and wound onto the $1^{\text {st }}$ and $2^{\text {nd }}$ stage cold plates of the GM cooler, respectively. 


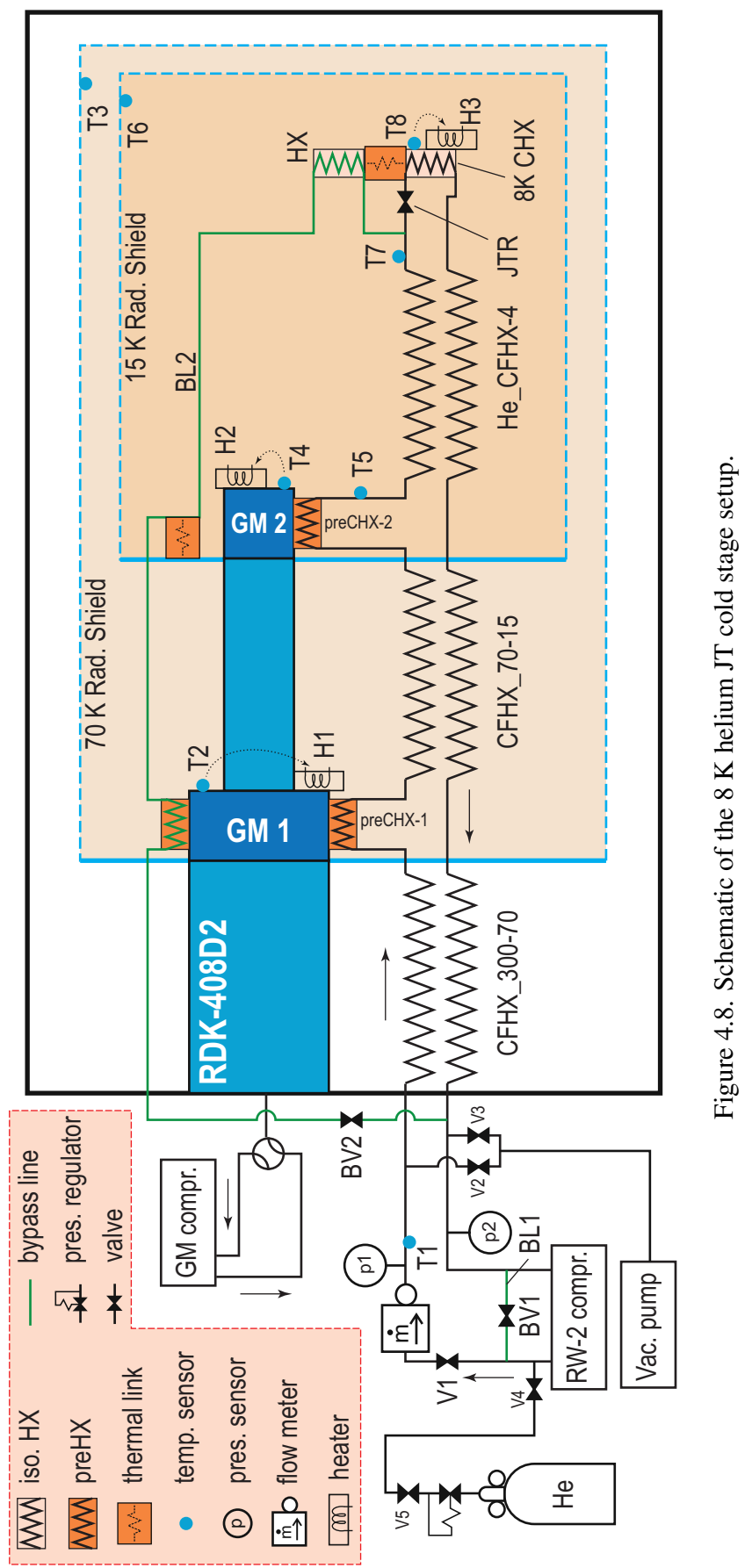


Two radiation shields were thermally linked to the two cold stages of the GM as well. Since the RDK-408D2 GM cooler is much more powerful than we need in this setup, resistance heaters $\mathrm{H} 1$ and $\mathrm{H} 2$ have to supply large heating powers to compensate the extra cooling power and keep the precooling temperatures at $70 \mathrm{~K}$ and $15 \mathrm{~K}$. MLI was only applied outside of the outer radiation shield and CFHX_300-70.

The flow in the JT cold stage was driven by a mechanical compressor, Leybold RW-2. This compressor is originally designed for a GM cooler and delivers almost a ten times higher flow rate than needed in this JT cold stage setup. Therefore, a flow bypass with a manual needle valve (BV1) was installed to connect the high-pressure to the low-pressure line. A mass flow meter (Bronkhorst EL-Flow ${ }^{\circledR}$, full-scale $150 \mathrm{mg} / \mathrm{s}$, calibrated at 14.5 bar with helium) measured the flow rate of fluid entering the high-pressure line of the JT cold stage. Two pressure sensors, p1 (GE PTX 1400, FS 25 bara) and p2 (GE PTX 5072 TB-A1, FS 25 barg), were used for measuring the high and low pressures at the warm end. Temperature sensors were placed at different positions as shown in Figure 4.8: T1, $\mathrm{T} 2$ and $\mathrm{T} 3$ are common diodes calibrated from room temperature to $50 \mathrm{~K}$ [77] measuring the room temperature, the operating temperature of the $1^{\text {st }}$ stage of the GM cooler and the $70 \mathrm{~K}$ radiation shield temperature; T4 and T6 are silicon diodes, DT670B-SD supplied by Lakeshore, mounted at the $2^{\text {nd }}$ stage of the GM cooler and the $15 \mathrm{~K}$ radiation shield; T5 and $\mathrm{T} 7$ are Lakeshore RoxTM sensors, recording the temperatures at the inlet and outlet of the high-pressure line of He_CFHX-4; T8 is a Lakeshore DT670A-CU, placed on the $8 \mathrm{~K}$ cold heat exchanger to measure the operating temperature of the JT cold stage.

During the cool-down process, the GM cooler is running and the flow in the JT cold stage has to be circulated to transfer the cooling power from the GM cooler to the JT cold stage and build a temperature gradient from the warm to the cold end. The flow has to be cooled down at least to below the inversion temperature (in this case about $36 \mathrm{~K}$ ) before it enters the JT restriction to create any cooling effect to continue cooling. However, if the gas is circulated through He_CFHX-4, it will take a very long time before the JT entrance is cooled to below $36 \mathrm{~K}$. That is because He_CFHX-4 was designed to have a very high effectiveness and very limited longitudinal thermal conduction. At start-up, the whole system is at $300 \mathrm{~K}$ and the GM cooler will quite quickly cool the respective stages to $70 \mathrm{~K}$ and $15 \mathrm{~K}$. However, the JT restriction and the $8 \mathrm{~K} \mathrm{CHX}$ will be at relatively high temperature and cool only by conduction and radiation. The circulating flow will not help in that respect. The precooled $15 \mathrm{~K}$ high-pressure flow will enter He_CFHX-4, but will be heated by the returning relatively warm flow. To fix this problem, a bypass line with a valve (BV2) was applied from the cold end of the high-pressure line to the warm end of the low-pressure line. BV2 will be open during the cool-down process so that most of the flow can go through this bypass line instead of returning through the low-pressure line of the CFHXs. The $15 \mathrm{~K}$ cooling power of the GM can thus be successfully transferred by the flow to the $15-8 \mathrm{~K}$ part. Once the $15-8 \mathrm{~K}$ part is cold enough, BV2 is closed, and the flow will go through the JT restriction creating cooling to further cool down. 


\subsubsection{Precooling subsystem}

The dimensions of the CFHXs and precooling heat exchangers are listed in Table 4.3, along with the pressure drops, outlet temperatures of the flows and the required cooling power at the two precooling stages.

Figure 4.9 shows the cooling capacity map of the Sumitomo RDK-408D2 GM cooler and the red dot marks the operating point, i.e. $70 \mathrm{~K}$ for the $1^{\text {st }}$ stage and $15 \mathrm{~K}$ for the $2^{\text {nd }}$ stage. It shows that the GM cooler can provide $10 \mathrm{~W}$ at $15 \mathrm{~K}$ and $70 \mathrm{~W}$ at $70 \mathrm{~K}$ which is more than sufficient for precooling the JT cold stage.

\subsubsection{5-8 K CFHX}

The CFHX model discussed in Sec. 4.3.2 was used to design the He_CFHX-4. Seamless $316 \mathrm{~L}$ steel tubes of various dimensions were investigated. The $1 / 8 * 0.028$ inch and

Table 4.3. Dimensions and estimated performance of the precooling subsystem.

\begin{tabular}{|c|c|c|c|c|c|c|}
\hline Components & $\begin{array}{c}D * \delta \\
\text { (Outer / Inner) } \\
\mathrm{mm}\end{array}$ & $\begin{array}{l}L \\
\mathrm{~m}\end{array}$ & $\begin{array}{c}E_{C F H X} \\
-\end{array}$ & $\begin{array}{c}\Delta p \\
\text { (high / low) } \\
\text { mbar }\end{array}$ & $\begin{array}{c}T_{\text {out }} \\
\text { (high / low) } \\
\text { K }\end{array}$ & $\begin{array}{c}\dot{Q}_{\text {preC }} \\
\mathrm{W}\end{array}$ \\
\hline CFHX_300-70 & $8 * 1 / 5 * 1$ & 1.5 & $90.17 \%$ & $7.96 / 76.92$ & $91.92 / 272.89$ & - \\
\hline preCHX-1 & $5 * 1$ & 0.8 & $99.86 \%$ & 1.27 & 70.03 & 12.11 \\
\hline CFHX_70-15 & $8^{*} 1 / 5^{*} 1$ & 2.4 & $89.34 \%$ & $2.83 / 15.25$ & 21.15 / 64.03 & - \\
\hline preCHX-2 & $2.5 * 0.4$ & 2.0 & $99.98 \%$ & 14.21 & 15.00 & 3.97 \\
\hline
\end{tabular}

RDK-408D2 Cold Head Capacity Map $(50 \mathrm{~Hz})$

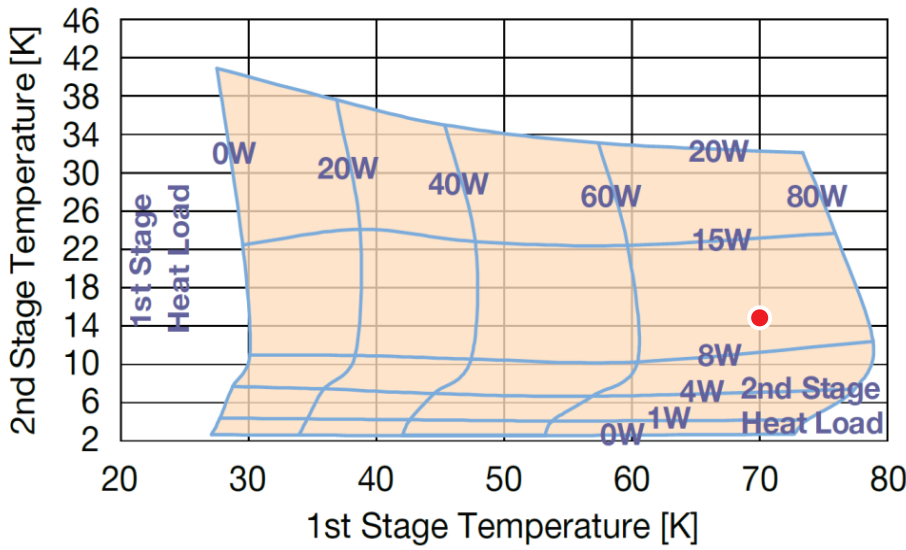

Figure 4.9. The cooling power map of the Sumitomo RDK-408D2 GM cooler. The red dot indicates the operating point for the JT cold stage setup. 


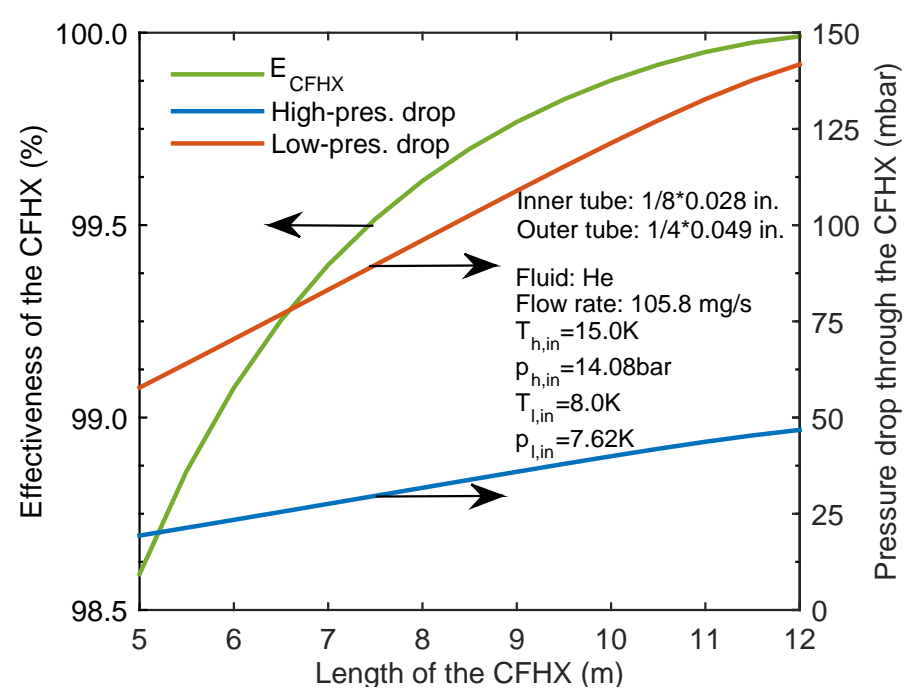

Figure 4.10. Effectiveness and pressure drops as a function of CFHX length for He_CFHX-4.

$1 / 4 * 0.049$ inch tubes were eventually selected, and the length of the CFHX was optimized as shown in Figure 4.10. In order to achieve an effectiveness higher than $99.8 \%$ in this critical CFHX, the CFHX has to be $9.24 \mathrm{~m}$. However, we chose about $25 \%$ margin and made the CFHX 11.5 m long, expecting an effectiveness of $99.97 \%$ and corresponding pressure drops of 45.4 and 137.6 mbar through the high-pressure and low-pressure channels, respectively.

\subsection{4 $8 \mathrm{~K} \mathrm{CHX}$}

The $8 \mathrm{~K}$ cold heat exchanger was designed to be a stainless tube winding on an isothermal copper cylinder. A semicircular groove was machined in a copper cylinder to provide more contact area (cylinder diameter $3 \mathrm{~cm}$ ). The tube was soldered on to the cylinder to have good contact. A numerical model similar to the CFHX model was built to find the required size of the $\mathrm{CHX}$. A $1 / 8 * 0.028$ inch tube with a length of $0.8 \mathrm{~m}$ was used resulting in 2.7 mbar pressure drop over the tube. By assuming an isothermal wall condition, the heat exchanger can achieve an effectiveness of $99.96 \%$.

\subsubsection{Joule-Thomson restriction}

The JT restriction has to provide a flow impedance that allows the helium flow (105.8 $\mathrm{mg} / \mathrm{s})$ isenthalpicly to expand from the high pressure (14.08 bar) to the low pressure (7.62 bar). In this setup, a capillary tube with an inner diameter of $203 \mu \mathrm{m}$ was used for this purpose. The Reynolds number in the tube is in the range of 17000-23000, and the Mach number is in the range of $0.115-0.223$. Therefore, the pressure drop through 


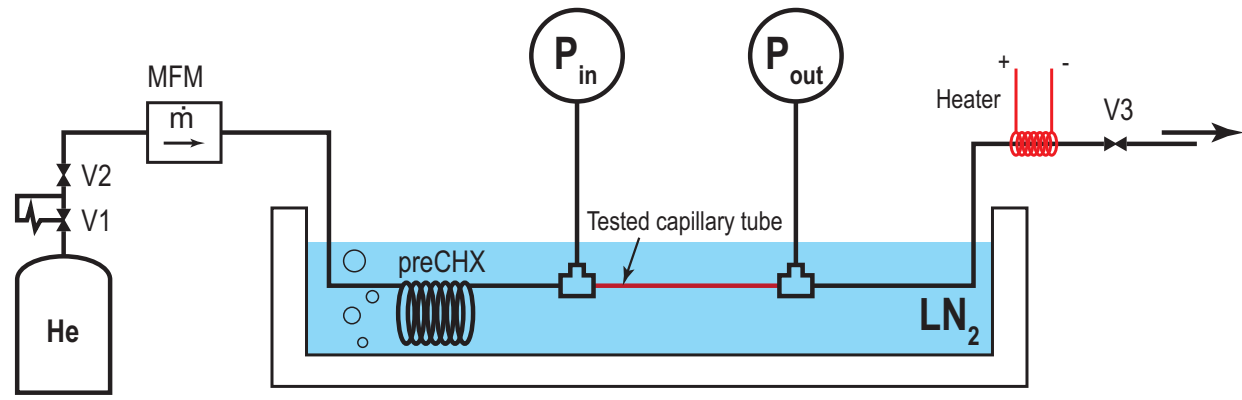

Figure 4.11. Schematic of the breadboard test setup to measure the roughness of the capillary tube.

the tube can be calculated by the Darcy-Weisbach equation in which the roughness of the inner surface of this capillary tube has to be known for evaluating the Darcy friction factor for the turbulent flow.

In order to find out how long the capillary tube should be to provide the desired impedance, a breadboard test as shown in Figure 4.11 was designed for measuring the pressure drops over the capillary tube at certain inlet pressures and flow rates. The incoming flow was regulated by valves V1 and V2 to the desired pressure, measured by a mass flow meter (MFM), and precooled in a liquid nitrogen (LN2) bath via an isothermal precooling heat exchanger (preCHX). The testing tube was immersed in the LN2 bath. In this way, the Reynolds number and Mach number of interest could be achieved. The pressures at the inlet and outlet were measured individually. The exhaust gas flow was heated up to room temperature and vented to the environment via valve V3 that controlled the flow rate. The pressure drops through the capillary tube were measured with different inlet pressures and mass flow rates.

In addition, a numerical model was built for simulating the fluid dynamics in the capillary tube by using the Darcy-Weisbach equation. This model was used to simulate the pressure drop test setup. Based on experimental data and simulation results the length of the capillary tube was chosen to be $14.8 \mathrm{~cm}$.

\subsubsection{Experimental results and discussion}

The JT cold stage system (without the compressor) was pumped and flushed several times to remove any contamination and kept at vacuum before connecting it to the RW-2 compressor via Aeroquip connectors. The RW-2 compressor was originally charged with helium at 16 bar (read from the pressure gauge on the compressor). To prevent the oil in the compressor contaminating the JT cold stage, the high-pressure line had to be connected first. It allowed the helium in the compressor to fill the JT cold stage via the oil separation system in the compressor. Since the volume in the JT cold stage is much smaller than that in the compressor, the equilibrium pressure stayed almost the same at 16 bar. Once the pressure was stabilized, the low-pressure line was connected. 
To cool the system, the GM cooler was first switched on with the JT compressor being off. The preCHX-1 and preCHX-2 were cooled down to $60 \mathrm{~K}$ and $5 \mathrm{~K}$ (controlled by the heaters on the GM, $\mathrm{H} 1$ and $\mathrm{H} 2$ ), and temperature gradients were created along CFHX_300-70 and CFHX_70-15. At the same time, T8 (the thermometer at the $8 \mathrm{~K}$ heat exchanger) was still above $180 \mathrm{~K}$. Then the JT compressor was switched on with BV1, BV2 and V3 open (see Figure 4.8). A flow of about $43.5 \mathrm{mg} / \mathrm{s}$ enters the high-pressure line of CFHX_300-70. Via preCHX-1 and preCHX-2, the flow "carried" the cooling power from the GM cooler to the next CFHX. Most of this flow returns via the bypass line since the JT restriction has a large flow resistance. The bypass line did not recover the cooling energy of the returning flow. Thus, the returning flow from the bypass line left the system at about the temperature of the first GM stage causing frost formation on the tube (outside of the vacuum bell jar). BV2 and V3 were tuned to have a proper flow rate. T8 started to drop while the mass flow rate also gradually increased because of the lower flow resistance at a lower temperature. The cool-down process was completed when T4, $\mathrm{T} 5, \mathrm{~T} 7$, and $\mathrm{T} 8$ were all bellow $20 \mathrm{~K}$.

Once the He_CFHX-4 and the 8K CHX were cooled down to $20 \mathrm{~K}$, BV2 was closed, and BV1 was tuned to adjust the flow rate and the pressures of the JT cold stage. The temperatures of the $1^{\text {st }}$ and $2^{\text {nd }}$ stages of the GM cooler were controlled at $70 \mathrm{~K}$ and $15 \mathrm{~K}$, respectively.

It was difficult to accurately determine how much charging pressure was required at room temperature since the internal volume of the compressor is unknown. The system was filled with helium gas or evacuated during the first operation to adjust it to the desired operation conditions: with a mass flow rate of $105.83 \mathrm{mg} / \mathrm{s}$ expanding from a high pressure of 14.08 bar to a low pressure of 7.62 bar. The aim was to have the actual operation as close as possible to the desired one.

With an average measured mass flow rate of $99.95(99.9 \pm 0.7) \mathrm{mg} / \mathrm{s}$, an average cooling power of $0.422(0.422 \pm 0.002) \mathrm{W}$ was measured at $7.98(8.00 \pm 0.25) \mathrm{K}$. The high and low pressures measured at room temperature were $14.96(14.95 \pm 0.05)$ bar and 7.31 $(7.30 \pm 0.05)$ bar respectively. The pressure drops through the CFHXs and preCHXs were evaluated based on the values in Table 3.5 with $105.8 \mathrm{mg} / \mathrm{s}$ and corrected according to the measured mass flow rate. The resulting pressure drops are $67.7 \mathrm{mbar}$ in the high-pressure line and 220 mbar in the low-pressure line. Then, the estimated pressures before and after the JT expansion were 14.89 bar and 7.53 bar. The cooling power at $8 \mathrm{~K}$ was measured for half an hour during which the temperature was stabilized in a range of $0.07 \mathrm{~K}$ and cooling power was stabilized in a range of $0.07 \mathrm{~W}$.

The parasitic losses including the radiation losses of the $8 \mathrm{~K} \mathrm{CHX}$ and the conduction losses through He_CFHX-4, the bypass line, and the electrical wires, were then calculated. The radiation loss from $8 \mathrm{~K}$ part to the $15 \mathrm{~K}$ surrounding was $14.3 \mu \mathrm{W}$, and the total conduction loss was $6.2 \mathrm{~mW}$.

By assuming He_CFHX-4 to be ideal, the calculated gross cooling power at the $8 \mathrm{~K}$ cold tip was $0.482(0.48 \pm 0.09) \mathrm{W}$ according to the measured mass flow rate and the 
Table 4.4. Operating pressures and performance comparison between the calculation and the measurement of the $8 \mathrm{~K}$ helium JT cold stage.

\begin{tabular}{lccc}
\hline Parameters & Opt. value in ideal case & Measured value & Deviation percentage \\
\hline$p_{h}$, bar & 14.08 & $14.96\left(14.89^{a}\right)$ & $+6.25 \%(+5.75 \%)$ \\
$p_{l}$, bar & 7.62 & $7.31(7.53)$ & $-4.07 \%(-1.18 \%)$ \\
$\Delta p_{h-l}$, bar & 6.46 & $7.65(7.36)$ & $+11.84 \%(+11.39 \%)$ \\
$T_{c}, \mathrm{~K}$ & 8.00 & 7.98 & $-0.25 \%$ \\
$\dot{Q}_{c}, \mathrm{~W}$ & 0.50 & $0.422(0.482)$ & $-15.60 \%(-3.60 \%)$ \\
$\dot{m}, \mathrm{mg} / \mathrm{s}$ & 105.83 & 99.95 & $-5.56 \%$ \\
\hline
\end{tabular}

\footnotetext{
${ }^{a}$ The values in the parentheses were evaluated according to the calculated pressure drops and the pressures measured at room temperature.
}

evaluated pressures. It is $14.2 \%$ higher than the measured cooling power and in the range of the design margin (25\% as indicated in Chapter 3 ).

Table 4.4 summarizes the operating pressures and performance results including cooling temperature, power, and mass flow rate. Compared to the design, the measurement shows $3.60 \%$ less flow rate and $11.84 \%$ more pressure difference between the high and low pressures. The flow impedance of the entire JT cooler is roughly $22.8 \%$ higher than calculated. One possible reason is that the length of the capillary JT restriction is over designed. In the numerical model that was used for choosing the JT restriction length, the entrance effect of the flow was not considered. However, at the relevant Reynolds numbers the entrance length can be 150 times the hydraulic diameter of the tube, yielding $3.04 \mathrm{~cm}$ in the total length of $14.8 \mathrm{~cm}$. According to Ref. [78], the friction factor at the entrance region should be increased by a minimum of $10 \%$ for design purposes.

Another reason is the contamination from the RW-2 compressor fouling the heat exchangers and the JT restriction and eventually clogging the flow. After the cooling power measurement, we kept the setup running at $8 \mathrm{~K}$ overnight. In the first 8.5 hours, the mass flow rate is quite stable. However, after that it started to decrease slowly. In 5.5 hours, the mass flow rate dropped by $2.2 \%$. The flow decreased further, and consequently the cooling power degraded. In 31 hours, the mass flow rate dropped to about $30 \mathrm{mg} / \mathrm{s}$, and the JT cold stage could no longer realize the $8 \mathrm{~K}$ and the temperature of the cold tip started to rise. In 63 hours, the mass flow rate decreased to $10 \mathrm{mg} / \mathrm{s}$. Unfortunately, the JT cold stage was found to be polluted by the lubricant oil from the RW-2 compressor. The oil was frozen in the high-pressure channel of the CFHX_300-70 and CFHX_70-15 causing the clogging issue.

\subsection{Conclusions}

Different JT cold stage configurations are discussed in this chapter. The parallel configuration is preferred in multi-stage sorption coolers. In Sec. 4.1.2, four configurations 
for the hydrogen cooler stage are considered, one of which the baseline as discussed in Chapter 3. The three alternative configurations perform better and further investigation on these designs is recommended.

A numerical model for a tube-in-tube CFHX was built for design purposes. Including the longitudinal conduction and radiation losses, the model simulates the heat transfer between the flows and the tubes and the pressure drops in the flow.

A full-scale $8 \mathrm{~K}$ helium JT cold stage was designed, fabricated and tested for demonstrating the helium JT cold stage in the METIS cooler chain. It obtained $0.422 \mathrm{~W}$ cooling power at $7.98 \mathrm{~K}$ which is above the requirement $(0.4 \mathrm{~W})$ and within the $25 \%$ design margin. Unfortunately, the setup was gradually clogged by oil contamination from the RW-2 GM compressor but the setup served its test purposes well. For long-term operation, an additional oil filtering system would have been required. 



\section{Sorption Compressor Design for the METIS Cooler}

In the baseline design of the METIS cooler that is discussed in Chapter 3, the sorption compressors are presumed to be equipped with an actively operated gas-gap heat switch. Although such a switch is attractive regarding efficiency, it severely complicates manufacturing the long compressor cells required for the METIS cooler. Therefore, in this chapter an alternative switchless compressor-cell design is investigated. The impact of this alternative compressor design on the cooler configuration for METIS cooler is discussed as well. 


\subsection{Limitations of the gas-gap heat switch}

Initially, most developments of sorption coolers were done for space applications [31, 34, 40] because of their unique advantages that have been already mentioned in Chapter 1 . In space applications, the coefficient of performance (COP) of the sorption JT cooler is of great importance because it directly relates to the size of the radiator on the spacecraft. The heat switch that makes and breaks the thermal contact of the sorption cell with the heat sink is, therefore, considered an essential part. Because of their high reliability, usually, gas-gap heat switches (GGHS) are used for this purpose [79-81].

To make an efficient and functional GGHS, a small gas gap is required between the adsorbent container and the heat sink (typically a few hundred microns [80]). The gap size is strictly defined and requires very high precision. The leak tightness and outgassing requirements for the gap space are comparable to those of ultra-high vacuum systems. In a small-scale cooler for space applications, where the compressor cell is relatively short and the number of cells is limited (e.g., 5 cells with a length of $10 \mathrm{~cm}$ for the $14.5 \mathrm{~K}$ hydrogen cooler in previous work [41]), the GGHS configuration is preferred for efficiency reasons. The METIS cooler, however, requires more than 50 cells, each $50 \mathrm{~cm}$ long. It is very difficult and expensive to realize a ultra-high vacuum quality narrow gap of $500 \mu \mathrm{m}$ over the full length of the cell. The defect rate in the production would not be acceptable. For the METIS cooler, the heat sink of the sorption compressor cells will be established by a pumped LN2 circuit with a large cooling capacity and, therefore, the compressor efficiency is less important than it is in space. Since the METIS cooler will be equipped with several tens of compressor cells, the manufacturability and costs of the cells become more a concern.

Furthermore, the selection of the operating gas-actuator pair in the GGHS is highly depended on the heat-sink temperature so that sometimes it makes the options quite limited. There are only two options for the gas-actuator pair in the GGHS of the METIS cooler: [nitrogen-activated carbon] and [hydrogen-metal hydride]. The former cannot build adequate conductance because of the limited conductivity of the nitrogen gas, which leads to a long cycle time and consequently to a large number of cells. The latter uses a metal-hydride actuator that, usually, operates above $60{ }^{\circ} \mathrm{C}$. It will require considerable input power to maintain the actuator at such a high temperature and the GGHS responding speed will be greatly compromised.

Therefore, an alternative compressor-cell design in which the GGHS is omitted was investigated, that greatly reduces the difficulty of manufacturing and assembly as well as the costs by sacrificing about $20 \%$ in efficiency.

\subsection{Switchless configuration}

In this alternative configuration for the METIS cooler, the GGHS is replaced by a fixed insulation layer (or insulator) as shown schematically in Figure 5.1. The insulation lay- 


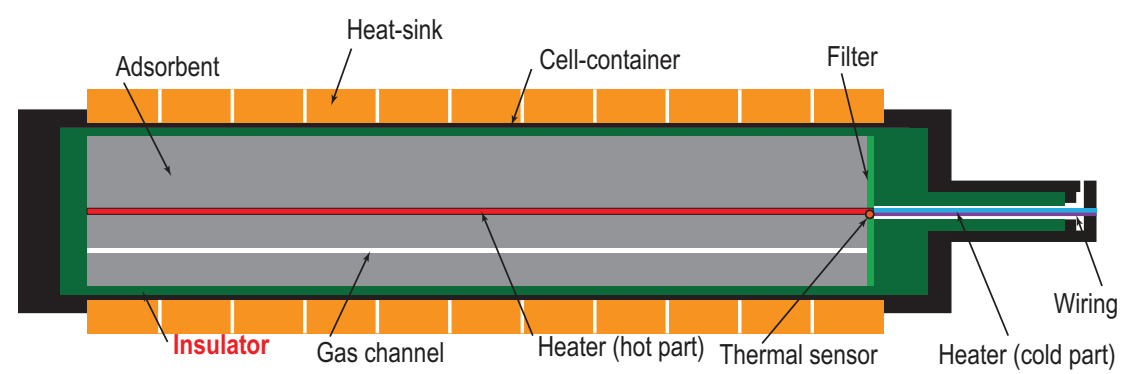

Figure 5.1. Schematic of a sorption compressor cooperating with a switchless insulation layer.

er material requires low conductivity, low heat capacity, low porosity and low thermal expansion coefficient. Furthermore, it should be an inert material. Kapton, Mylar, and Teflon are qualified candidates [82]. Kapton has the best thermal properties followed by Mylar, whereas Teflon is commercially available in a variety of geometries. Nevertheless, the solid insulation layer will have a much higher conductance than the GGHS in the OFF state. A significant amount of heat will leak to the heat sink during the desorption process (a-b-c in Figure 2.5). One can simply increase the thickness of the insulation layer to reduce this conduction loss, but thicker insulation also slows down the cooling process in the adsorption process (c-d-a) resulting in a longer cycle time and more cells required. Another way to decrease the conduction loss is rapid and short heating. However, short high-power pulse heating will generate a large radial temperature gradient in the adsorbent, resulting in a degradation of the performance. The insulation layer is inside the cell container that is thermally attached to the heat sink. Therefore, the wall of the container will experience a relatively small temperature swing. This reduces the parasitic loss in the thermal mass of the container. This is specifically relevant for a high-pressure compressor such as the neon compressor in the METIS cooler in which the container is quite thick. Considerable input power is needed to thermally cycle the container material in case of a GGHS.

In general, this switchless configuration will have a lower production cost and will be easier to assemble. With well-optimized dimensions, the switchless configuration is expected to require more input power than the GGHS configuration, but fewer cells since the cycle time may be reduced.

\subsection{Dynamic model}

\subsubsection{Analytical description}

In order to understand the thermal behavior of the switchless configuration cell and to predict its performance, a dynamic model was built. Although a quasi-static model was built and used for the preliminary design of the METIS cooler (Chapter 3), it is not accurate for the dynamic operation of the switchless configuration. Because of the short 
high-power pulsed heating during the desorption process, a large radial temperature gradient is expected. The assumption of a uniform temperature in the quasi-static model will give quite a significant deviation from the real behavior. Furthermore, the quasi-static model cannot provide an evaluation of the cycle time of the sorption compressor thus the required number of cells cannot be estimated with a good accuracy. The assessment of the cycle time by the dynamic model will be more reliable than that estimated it by using a simplified RC model.

This dynamic model is 1-dimensional assuming a relatively large aspect ratio of the compressor cell (in the METIS cooler design it will be more than 30), and only the radial temperature gradient is simulated. The dynamics of the fluid mass transfer is neglected. That means the momentum equation will not be solved in this model. The pressure drop through the cell will be checked later. Quasistatic sorption behavior is assumed, which implies that no dynamics is considered for the adsorption equilibrium. Locally, the amount of gas adsorbed or desorbed instantaneously follows the local temperature and pressure, according to the adsorption isotherms. Thus, the behavior of the sorption compressor cell is reduced to a thermal conduction problem interacting with the adsorption on the adsorbent material.

Figure 5.2 shows a cross-sectional view of the switchless sorption compressor cell for the dynamic model. The cylindrical cell is divided into small tubular control volumes in the radial direction. In this thesis referred to as adsorption mixture, the adsorption part is considered as a three-component mixture consisting of the porous solid adsorbent material, the free working fluid that is not adsorbed and the adsorbed working fluid that is attached on to the surface of the adsorbent material in a condensed state. The outer

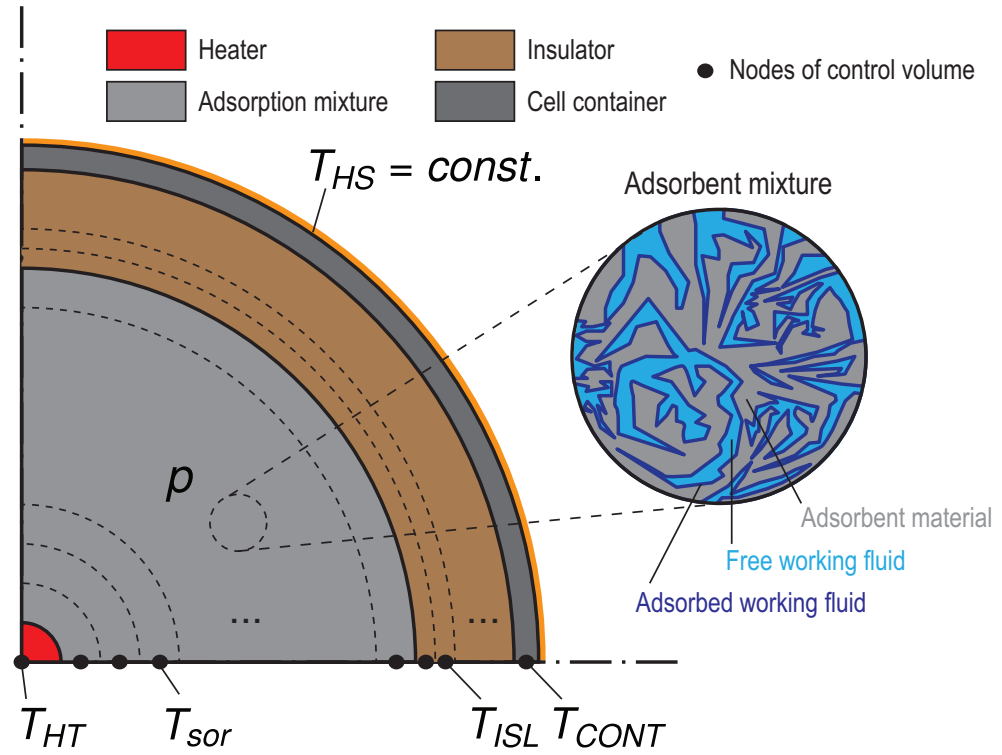

Figure 5.2. Cross-sectional view of the switchless sorption compressor in the dynamic model. 
surface of the cell container is assumed to be fixed at the heat-sink temperature $T_{H S}$.

The fundamental partial differential equation of the energy balance for thermal conduction in a solid material is given by,

$$
\rho c \frac{\partial T}{\partial t}=\nabla \cdot(k \nabla T)+S
$$

Here $S$ is the source term, the heat locally produced per unit of volume (in $\mathrm{W} / \mathrm{m}^{3}$ ). Eq. 5.1 can directly be applied to the heater, the insulation layer and the cell container within a 1-D cylindrical coordinates as follows,

$$
\begin{aligned}
& \text { Heater: } \quad \rho_{H T} c_{H T} \frac{\partial T}{\partial t}=\frac{1}{r} \frac{\partial}{\partial r}\left(k_{H T} r \frac{\partial T}{\partial r}\right)+\frac{4 \dot{Q}_{H T}}{\pi D_{H T}^{2} L} \\
& \text { Insulation layer: } \quad \rho_{I S L} c_{I S L} \frac{\partial T}{\partial t}=\frac{1}{r} \frac{\partial}{\partial r}\left(k_{I S L} r \frac{\partial T}{\partial r}\right) \\
& \text { Cell Container: } \quad \rho_{\mathrm{CONT}} c_{\mathrm{CONT}} \frac{\partial T}{\partial t}=\frac{1}{r} \frac{\partial}{\partial r}\left(k_{\mathrm{CONT}} r \frac{\partial T}{\partial r}\right)
\end{aligned}
$$

$\dot{Q}_{H T}$ is the heating power of the heater in watt, $D_{H T}$ is the diameter of the heater, and $L$ is the length of the heater which is presumed to equal the length of the sorption compressor cell.

The energy conservation of the adsorbent mixture is more complicated, and can be expressed as,

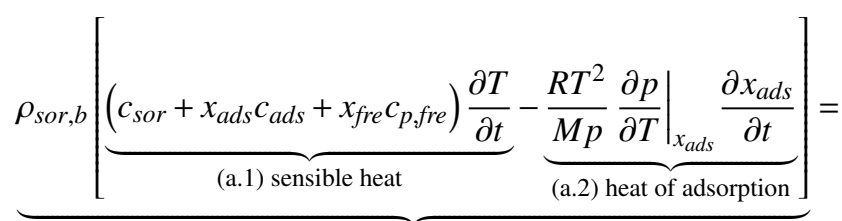

$$
\begin{aligned}
& \text { (a) Unsteady term (enthalpy change) } \\
& \underbrace{\nabla \cdot\left(k_{\text {eff }} \nabla T\right)}-\underbrace{\rho_{\text {sor }, b} \nabla \cdot\left(x_{\text {fre }} h_{f r e} \vec{V}\right)} \\
& \begin{array}{ll}
\text { (b) Diffusion term } & \text { (c) Convection term }
\end{array} \\
& \text { (heat flow due to the conduction) (enthalpy flow due to the convection) }
\end{aligned}
$$

where $\rho_{s o r, b}$ is the bulk density of the adsorbent material that is given by $\rho_{s o r, b}=\rho_{s o r, t}(1-$ $\alpha), \vec{V}$ is the velocity field of the free working fluid and $k_{\text {eff }}$ indicates an effective thermal conductivity of the adsorption mixture. The unsteady term that represents the enthalpy change is composed of the sensible heat due to the temperature change and the heat of adsorption. This is similar to Eq. 2.20. On the right side of Eq. 5.5, there are the diffusion term and the convection term. The former represents the heat flow due to the thermal conduction. In a 1-D cylindrical coordinates it is,

$$
\nabla \cdot\left(k_{e f f} \nabla T\right)=\frac{1}{r} \frac{\partial}{\partial r}\left(k_{e f f} r \frac{\partial T}{\partial r}\right)
$$


The latter term on the right side of Eq. 5.5 indicates the enthalpy flow due to the mass redistribution of the free working fluid, that is determined by the velocity field and the specific enthalpy of the free working fluid in the domain. An approximation has to be made, since the dynamics of the fluid transfer is not of interest in this model. It is assumed that the mass transfer only happens in the radial direction for the compression and decompression phases (no flow in our out of the cell), whereas it is in the axial direction for the in-flow and out-flow phases. The convection term approximately equals (see Appendix),

$$
\rho_{\text {sor }, b} \nabla \cdot\left(x_{f r e} h_{f r e} \vec{V}\right) \approx \begin{cases}-\rho_{\text {sor }, b} h_{l, H S} \frac{\partial x_{t o t}}{\partial t} & \text { in-flow phase } \\ -\rho_{\text {sor }, b} h_{f r e} \frac{\partial x_{t o t}}{\partial t} & \text { other phases }\end{cases}
$$

where $h_{l, H S}$ is the specific enthalpy of the incoming flow that is with the low pressure $p_{l}$ and heat-sink temperature $T_{H S}$ whereas $h_{f r e}$ is the specific enthalpy of the local free working fluid.

Because the momentum conservation equation is not of interest in this model, it is necessary to find an equation including the pressure to close the equation system. That is,

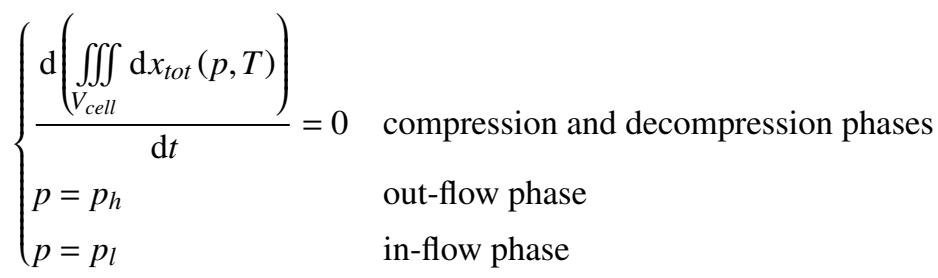

In the compression and decompression phases, the pressure in the cell is calculated by the conservation of the total mass of the working fluid in the cell. During the outflow and in-flow phases, the pressures are assumed to equal the high and low pressures, respectively.

Furthermore, the boundary condition is given by the assumption of a constant heatsink temperature $T_{H S}$ at the outer surface of the cell container. As shown in Figure 5.2, the finite-volume method is used in this dynamic model. All parts of the sorption compressor cell are divided into tubular finite control volumes. The partial-differential equations Eq. 5.2-5.5 are converted into a series of discretized equations. Combined with Eq. 5.8 and the boundary condition, the equation system is solved by the non-linear solver in MATLAB for each time step. The time derivative of the total working fluid mass integrated over the cell volume yields the mass flow rate produce by the sorption compressor cell.

\subsubsection{Simulations and discussions}

Typical simulation results of this dynamic sorption compressor model for the helium compressor in the METIS cooler are presented in Figure 5.3. In this simulation, a heat-sink temperature of $70 \mathrm{~K}$ is assumed, the carbon diameter is $14.5 \mathrm{~mm}$, the thickness of the 


\subsection{Dynamic model}

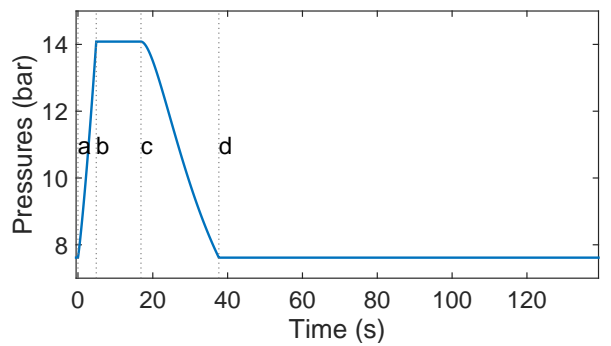

(a)

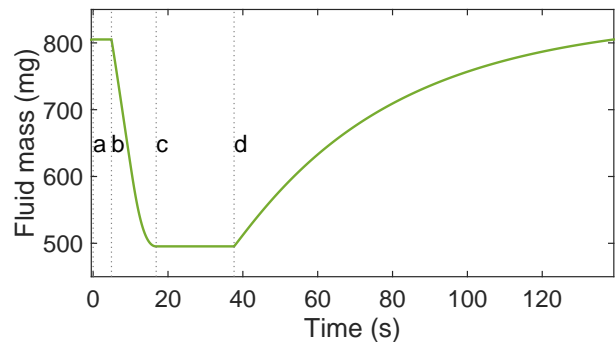

(c)

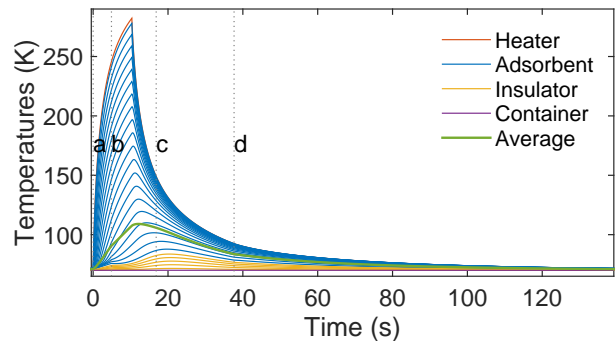

(e)

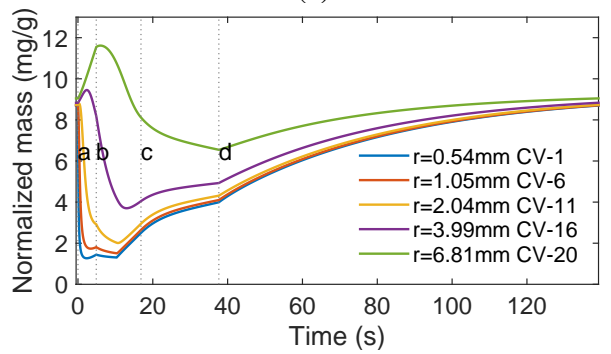

(g)

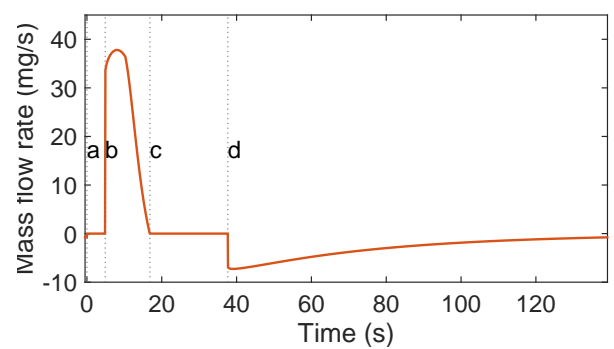

(b)

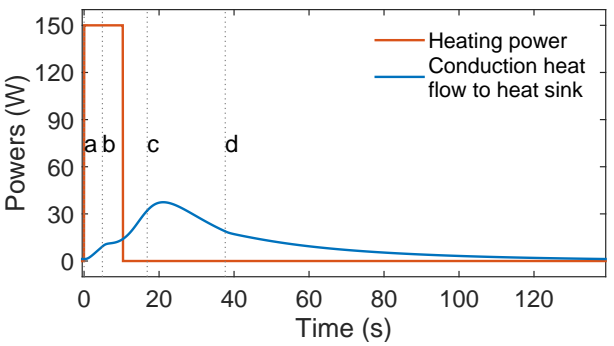

(d)

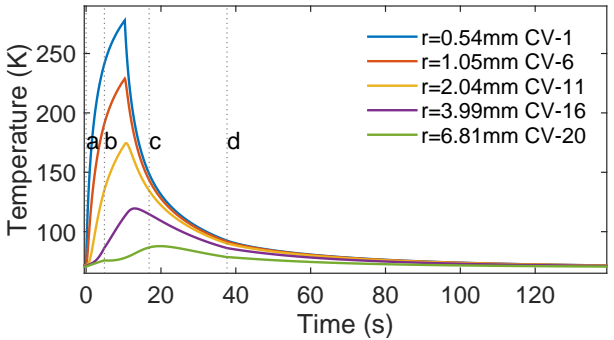

(f)

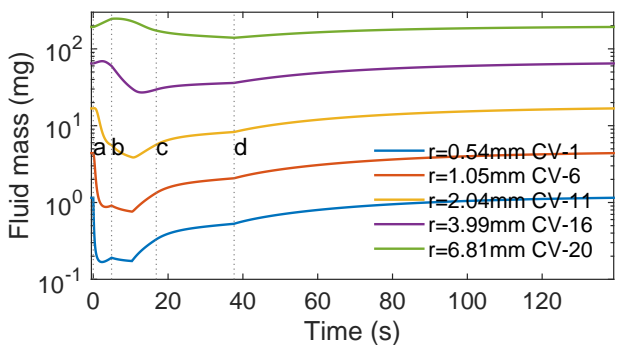

(h)

Figure 5.3. Typical simulation results of the dynamic sorption compressor model for the helium compressor in the METIS cooler. The vertical dashed lines split the whole cycle into 4 phases as described in Sec. 2.2.1. (a) Pressure in the cell; (b) Mass flow rate generated by the cell (positive value for out-flow and negative value for in-flow); (c) Total fluid mass in the cell; (d) Heating power of the heater and the heat flow into the heat sink; (e) Temperatures of the control volumes and the average temperature of the adsorbent mixture; (f) Temperatures of selected adsorbent mixture control volumes. $r$ is the radial coordinate of the control volume (CV). CV-1 represents the most inner one whereas CV-20 for most outer one; (g) Normalized fluid mass in the selected control volumes; (h) Fluid mass in the selected control volumes. 
Kapton insulation is $1.25 \mathrm{~mm}$, and the thickness of the container wall is $250 \mu \mathrm{m}$. A highpower pulse heating of $150 \mathrm{~W}$ is applied. The heating lasts $10.33 \mathrm{~s}$ as shown in Figure 5.3 (d), until the average temperature of the carbon reached the optimum high temperature of 107.5 K that is presented in Chapter 3. The total cycle time is $139.13 \mathrm{~s}$.

The compression phase lasts $4.89 \mathrm{~s}$ in which the pressure in the cell rises rapidly from the initial low pressure of 7.62 bar to a high pressure of 14.08 bar (see Figure 5.3 (a)). A large temperature gradient along the radial direction is built in the cell (see Figure 5.3 (e)). The inner carbon is heated up to $278 \mathrm{~K}$ while the outer part is still below $78 \mathrm{~K}$ (see Figure 5.3 (f)). The fluid mass contained in the carbon control volumes depends on the changes in the temperature and pressure. As shown in Figure $5.3(\mathrm{~g})$, at the beginning of the heating the inner carbon desorbs quickly due to the rapid rise in temperature while the outer carbon adsorbs because of the pressure increasing. After about $2 \mathrm{~s}$ heating, the inner carbon starts adsorbing instead of desorbing the working fluid because the effect of the pressure increase at that location becomes more dominant (see the blue and red lines in Figure $5.3(\mathrm{~g})$ ). In contrast, the outer carbon starts to desorb once the effect of the rising temperature is more dominant (see the purple and green lines in Figure $5.3(\mathrm{~g})$ ). Figure $5.3(\mathrm{~h})$ presents the absolute value of the fluid mass in the selected carbon control volume, indicating that the fluid is transferred from the inside to the outside during the compression phase.

Once the pressure reaches the high pressure, the high-pressure check valve opens and the working fluid is allowed to flow out of the cell at a constant high pressure (see Figure 5.3 (a) and (b)). The out-flow phase can be divided into two periods. In the first $5.44 \mathrm{~s}$ of this phase, the heating is still on, and a mass flow of about $34-38 \mathrm{mg} / \mathrm{s}$ is generated. Note the temperature of the outer carbon has a plateau because of the energy balance between the conduction heat, the enthalpy flows and the heat of adsorption. As a result, a similar plateau is found in the heat flow into the heat sink as shown in Figure 5.3 (d). The second period starts with switching off the heating and then the out flow lasts another $6.51 \mathrm{~s}$. Once the heating is stopped, the inner carbon cools down quickly transferring energy to the outside. The temperature wave progresses outwards rapidly. Thus, the outer carbon temperature starts to increase and desorb the working fluid creating a sudden rise in the mass flow rate as shown in Figure 5.3 (b). As the outer carbon is heated, the heat flow rate into the heat sink increases.

When the desorption in the cell can no longer maintain the high-pressure at 14.08 bar, the high-pressure check valve closes and the flow out of the cell into the buffer at that point is zero. Then, the decompression phase begins. In this phase, the pressure in the cell drops as the temperature wave further progresses outwards. During the entire decompression phase, the outer carbon is gradually desorbing the fluid while the inner carbon is adsorbing.

After $20.81 \mathrm{~s}$ of decompression, the in-flow phase starts which is the longest phase of the four. It lasts $101.48 \mathrm{~s}$ bringing the average temperature of the carbon to $71 \mathrm{~K}$. The in-flow rate is much less than the out-flow rate, but it lasts much longer. Obviously, the 
time integrals of the out-flow and in-flow rate are equal.

\subsection{Experimental validation of the dynamic model}

\subsubsection{Experimental setup}

The dynamic model is preliminarily validated by two experiments [83, 84]. The first one, setup 1 , is cycling a closed sorption compressor cell, building up the pressure and then depressurizing it. The second one, setup 2, combines check valves with the compressor cell to generate a mass flow at a certain pressure difference. In both experiments, the resulting pressure and mass flow rate were measured as a function of time. The results were compared with the simulation results given by the dynamic model, to confirm its validity.

A sorption compressor cell built in a previous project shown in Figure 5.4 [41] was used in both experiments. It is a sorption compressor cell designed for a hydrogen cooler. It uses a GGHS configuration, with a $100 \mu \mathrm{m}$ gas gap. To simulate the switchless configuration, the gas gap was filled with conductive gas at a steady pressure that then formed the insulation layer. The original check valves were designed to satisfy space application requirements for the specific pressure and mass flow range of the hydrogen cooler. They were removed in setup 1, and replaced in setup 2 by Swagelok high-purity commercial check valves (6LV-CW4BW6M-NE, see Sec. 6.2.3) which are cheaper and more flexible in an experimental test setup.

A schematic overview and a photograph of setup 1 are presented in Figure 5.5 (a) and (b). The sorption compressor cell is mounted on an aluminum platform that was immersed in a liquid-nitrogen bath during the experiment. The compressor cell is connected to a gas bottle, the vacuum pump, and a buffer volume through valves. The system was pumped and flushed for several times before being filled to a certain initial pressure (around 4-

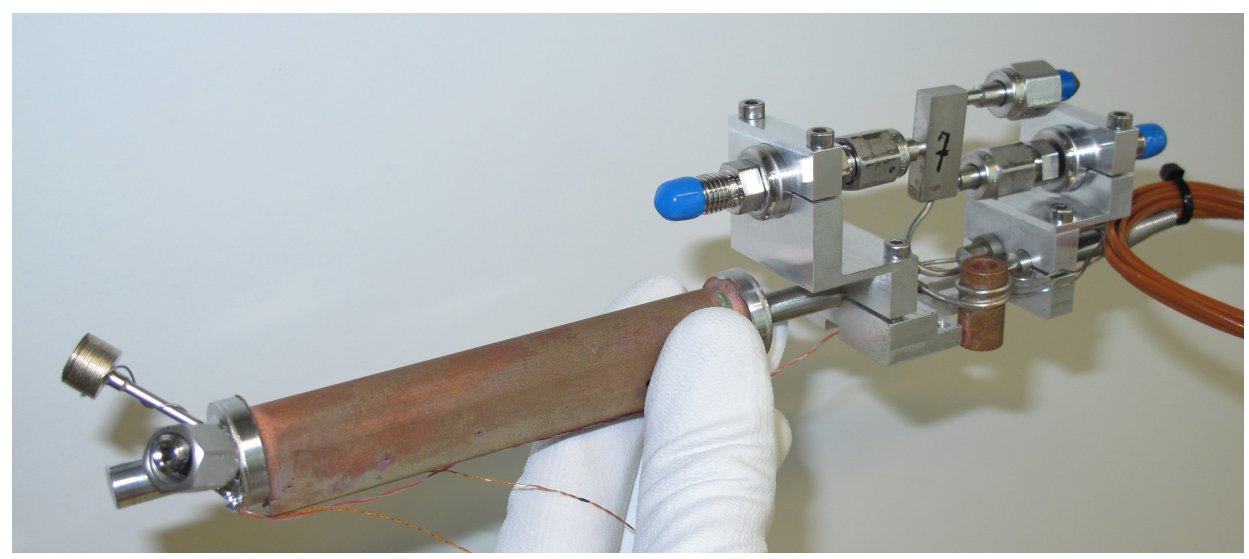

Figure 5.4. Sorption compressor cell for $14.5 \mathrm{~K}$ hydrogen cooler [41]. 


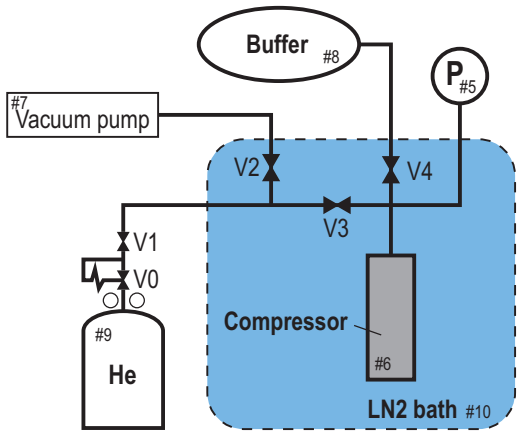

(a)

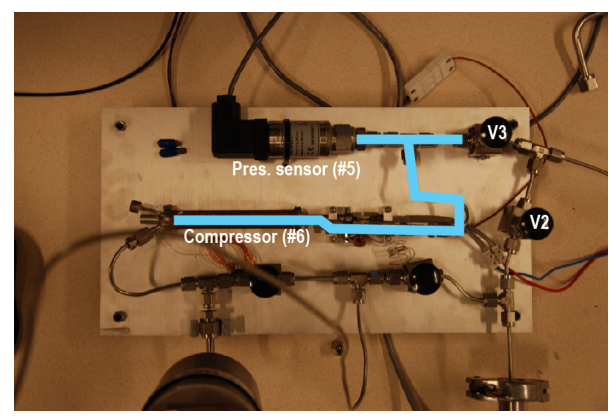

(b)

Figure 5.5. (a) Schematic overview of setup 1: V0-V4.Valves; \#5. Pressure sensor; \#6.Compressor cell; \#7.Vacuum pump; \#8.Buffer volume; \#9.Gas bottle. (b) Photograph of setup 1, with buffer removed.

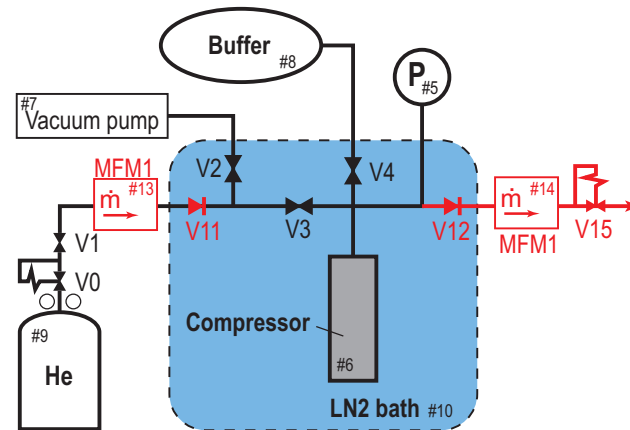

(a)

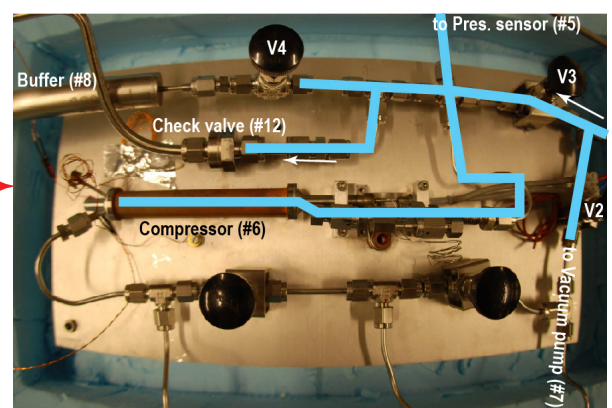

(b)

Figure 5.6. (a) Schematic overview of setup 2: V0-V4.Valves; \#5. Pressure sensor; \#6.Compressor cell; \#7.Vacuum pump; \#8.Buffer volume; \#9.Gas bottle; V11-12.Check valves; \#13-14, Mass flow meter; V15.Relief valve. (b) Photograph of setup 2.

10 bar). With all valves closed, the compressor cell was cycled by cyclic heating. A pressure wave was thus established and monitored in the volume composed of the compressor cell and some additional tubing, indicated by the blue line shown in Figure 5.5 (b).

Setup 2 is presented in Figure 5.6. Two check valves (V11 and V12), two massflow meters (\#13-14) and a relief valve (V15) were added based on Setup 1. During the operation, the compressor pumped the working fluid from a low pressure to a high pressure. The in-flow was delivered by a gas bottle, and the low pressure was controlled by a pressure regulator. The high pressure was regulated by the relief valve, and the out-flow was vented to atmosphere. The flow direction was secured by the check valves. Not only pressure, but also the in-flow rate and the out-flow rate were carefully measured during the experiment and compared with the simulation results obtained from the dynamic model.

For the purpose of model validation, it is essential to know the parasitic void volume $V_{\text {void,p }}$ in the experimental setups. The parasitic void volume consists of the volume in 
the tubing, in the pressure sensor, and in the valves. The total void volume $V_{\text {void,tots }}$ is the sum of this parasitic void volume and the void volume in the adsorbent $V_{\text {void,sor }}$.

$$
V_{\text {void,tot }}=V_{\text {void,para }}+V_{\text {void,sor }}
$$

To determine the parasitic void volume, a buffer with a known volume was connected through a valve V4. The system was pumped to vacuum, then filled with helium to an initial pressure $p_{i}$, with V4 closed. Then V4 was opened, letting the helium flow into the buffer volume resulting in a pressure drop to a final pressure, $p_{f}$. In this process, the mass of helium was constant,

$$
\begin{aligned}
m_{\mathrm{He}} & =m_{\text {sor }} x_{a d s}\left(T, p_{i}\right)+\frac{p_{i} V_{\text {void,tot }} M}{R T} \\
& =m_{\text {sor }} x_{\text {ads }}\left(T, p_{f}\right)+\frac{p_{f}\left(V_{\text {void,tot }}+V_{B F}\right) M}{R T}
\end{aligned}
$$

At the room temperature, the adsorption amount of helium in the saran carbon is negligible therefore Eq. 5.10 can be simplified into,

$$
p_{i} V_{\text {void,tot }}=p_{f}\left(V_{\text {void }, \text { tot }}+V_{B F}\right)
$$

Solving Eq. 5.11, the parasitic void volume can be readily determined,

$$
V_{\text {void,para }}=\frac{p_{f} V_{B F}}{p_{f}-p_{i}}-V_{\text {void,sor }}
$$

The measurements were performed by using different initial filling pressures $p_{i}$ from 5-15 bar. The parasitic void volumes of both setups were measured, that are $1.87 \pm 0.13 \mathrm{cc}$ for setup 1 and $2.00 \pm 0.13 \mathrm{cc}$ for setup 2 .

\subsubsection{Experimental results and discussion}

Typical measurement results for setup 1 are presented in Figure 5.7 with the corresponding simulated results. A square heating pulse of $30 \mathrm{~W}$ was used in the measurement for different heating times. In the helium measurement, the pressure recording matches the simulated pressure very well for short heating times, as shown in Figure 5.7 (a). However, after a series experiment, the liquid nitrogen was run out that part of the cell was out of the bath in the later cases that the heating time were increased to $12 \mathrm{~s}$ and $16 \mathrm{~s}$, resulting an increase in the heat-sink temperature. Because of that, the cell did not adsorb as much gas as it was assumed in the simulation. So the measured pressure has a significant deviation from the simulated values for these two cases.

In the case of neon as the working fluid, more gas can be adsorbed on the saran carbon compared to helium at the same liquid-nitrogen temperature. Therefore, the initial filling pressure was around 5 bar and the heating time was limited up to $8 \mathrm{~s}$, to prevent a too high pressure during the operation. The neon measurement results match the simulated 


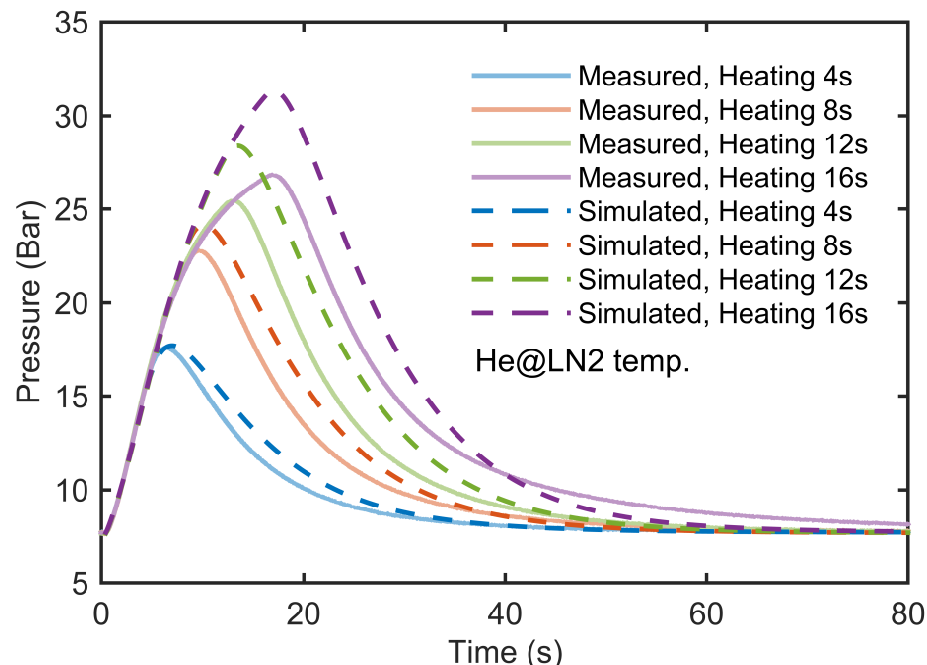

(a)

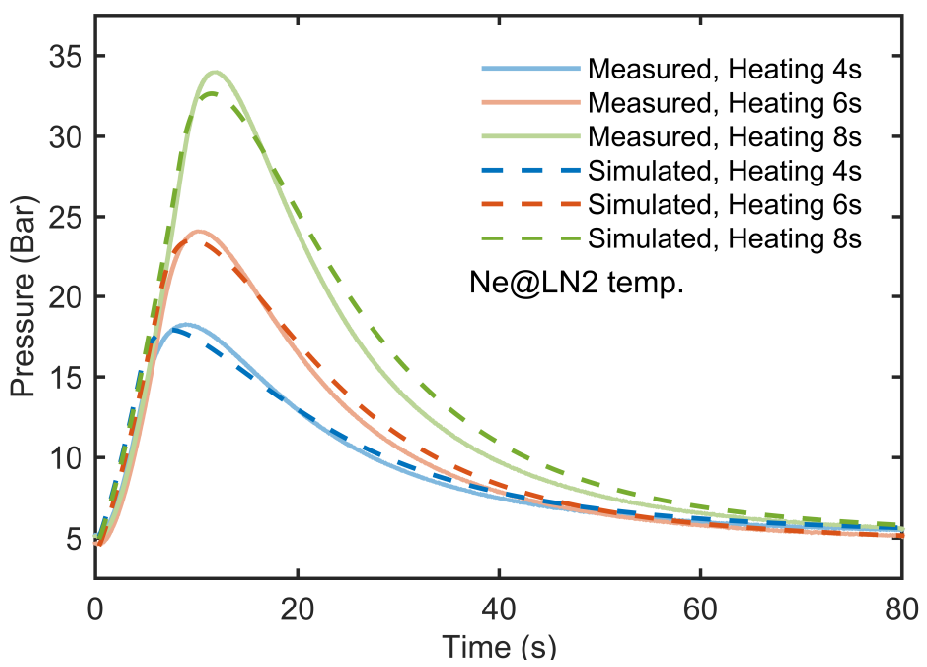

(b)

Figure 5.7. Measured pressure in setup 1 compared to the simulations. A square heating pulse of $30 \mathrm{~W}$ was applied in all cases. (a) The working gas is helium, with initial filling pressure is about 7.7 bar. Four runs with different heating times from $4 \mathrm{~s}$ to $16 \mathrm{~s}$ are presented. (b) The working gas is neon, with initial filling pressure is about 5.0 bar. Three runs with different heating times of $4 \mathrm{~s}$, $6 \mathrm{~s}$ and $8 \mathrm{~s}$ are presented. 


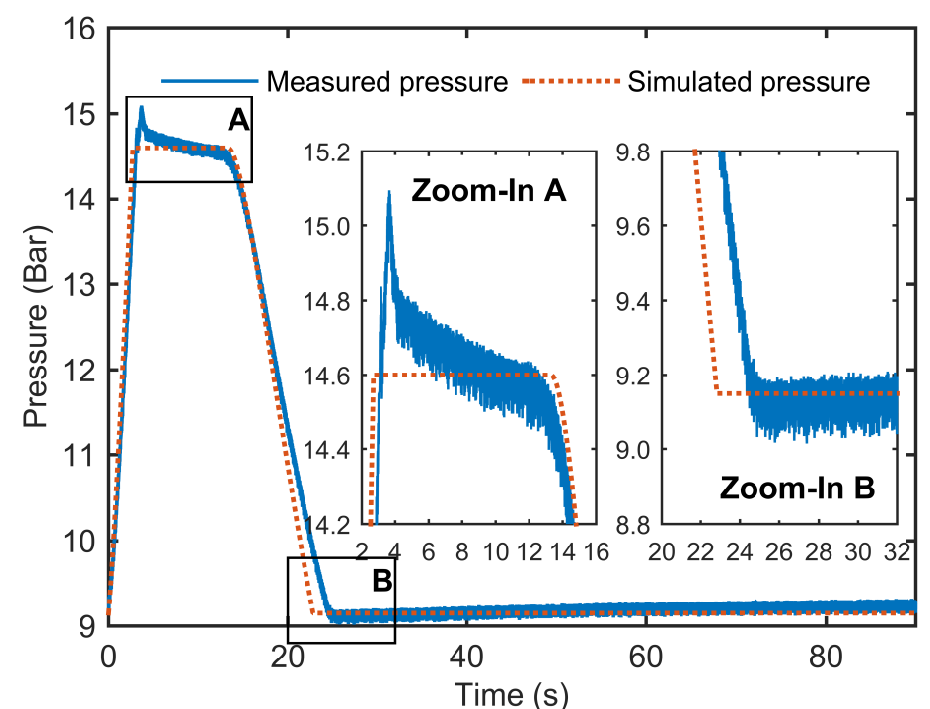

(a)

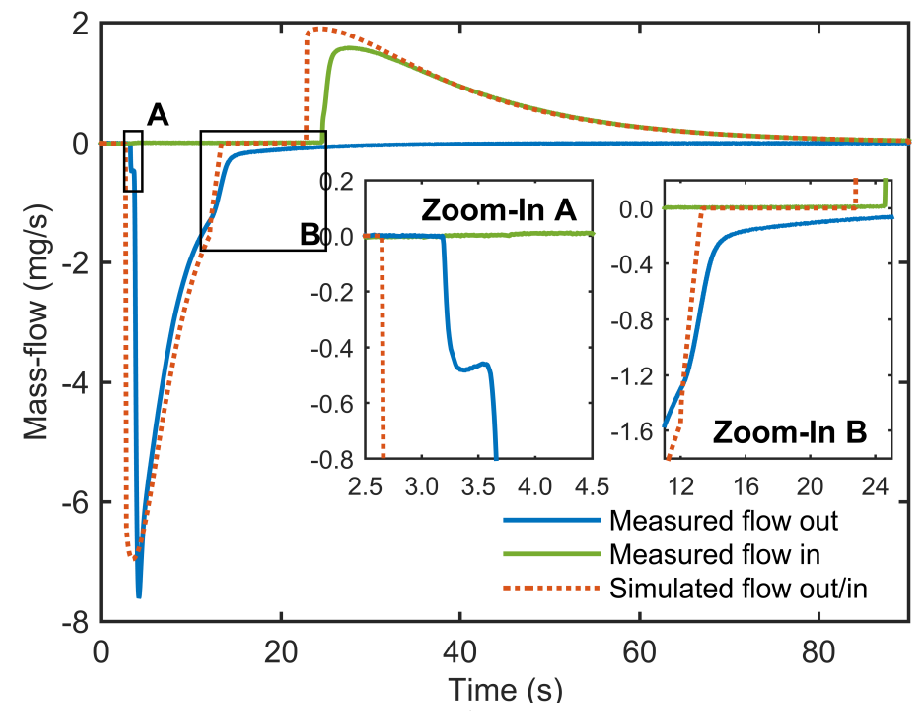

(b)

Figure 5.8. Measured pressure in setup 1 compared to the simulations. A square heating pulse of $30 \mathrm{~W}$ was applied in all cases. (a) The working gas is helium, with initial filling pressure is about 7.7 bar. Four runs with different heating times from $4 \mathrm{~s}$ to $16 \mathrm{~s}$ are presented. (b) The working gas is neon, with initial filling pressure is about 5.0 bar. Three runs with different heating times of $4 \mathrm{~s}$, $6 \mathrm{~s}$ and $8 \mathrm{~s}$ are presented. 
results very well also in the cool-down phase. The measurement results for neon match the simulated results very well, as shown in Figure 5.7 (b).

Figure 5.8 presents the pressure and flow rate in setup 2 for a single cycle operation. The working gas was helium. The initial filling pressure was 9.2 bar; the relief valve V15 was set at 14.5 bar and the working gas was helium. The pulse heating was $30 \mathrm{~W}$ for $12 \mathrm{~s}$ resulting in a rapid increase of the pressure in the compressor. The cracking pressure of the check valve appeared to be smaller than that of the relief valve so that the check valve opens first at around 14.8 bar while the relief valve was still closed. A small out-flow step was first measured at that moment and is shown in "Zoom-In A" Figure 5.8 (b). The relief valve V15 opened when the pressure increased up to 15.1 bar; a peak outflow was then generated accompanied with a pressure drop back to 14.5 bar, the relief valve setting value. Because of the pressure drop through the valves, the pressure in the cell was higher than 14.5 bar during the out-flow phase and lower than 9.2 bar during the in-flow phase. Furthermore, as shown in "Zoom-In B" Figure 5.8 (b), the check valve could not close immediately and had a leakage.

However, in the simulation the check valve and the relief valve behaviors were not taken into account, and the high and low pressures were set to be 14.60 bar and 9.15 bar. Consequently, the simulated out-flow starts about $1 \mathrm{~s}$ earlier than that in the real experiment. The simulated out-flow rate has a lower peak but the value is larger in average compared to the measurement. The simulated in-flow starts $2 \mathrm{~s}$ earlier than the measurement, and has a peak flow rate of $18.7 \mathrm{mg} / \mathrm{s}$. The simulation curve meets the measurement when $t=34 \mathrm{~s}$ and a good match is achieved till the end of the cycle. By integrating the mass flow over a cycle, a total amount of $34.2 \mathrm{mg}$ helium gas was found to be pumped in a cycle in the measurement compared to $40.4 \mathrm{mg}$ in the simulation. The relative error is $18.23 \%$. The deviation between the measurement and simulation is mainly caused by

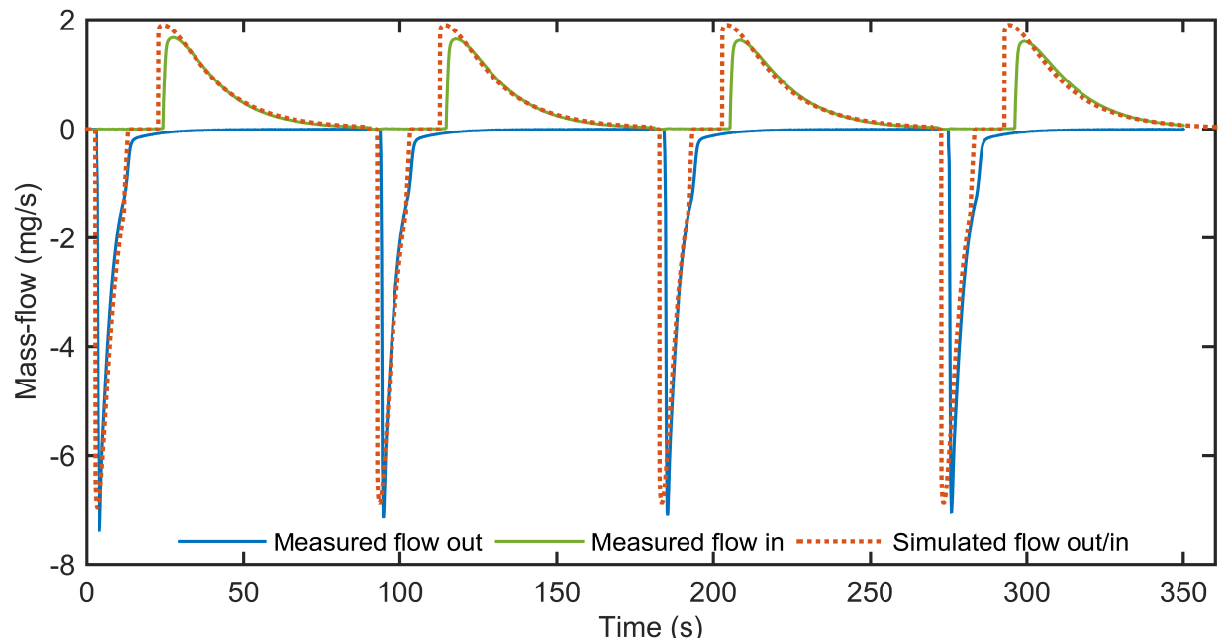

Figure 5.9. Measured flow rate in setup 2 compared to the simulation, four complete cycles. 
the non-ideality of the valves. Therefore, reasonable margins related to the check-valves should be considered by using this model to design a sorption compressor.

Another measurement included multiple cycles with a fixed cycle time of $90 \mathrm{~s}$. The measured mass flow rate versus time is shown in Figure 5.9. Note that the mass-flow peak decreased slightly each cycle. That is due to the liquid-nitrogen level dropping during the experiment. The heat-sink temperature slowly increases that results in a slight performance degradation. However, the measurement results agree with the simulation within reasonable errors mainly caused by non-ideality of the valves.

In summary, the dynamic model is validated by the experiments, and can be used for predicting the performance of the sorption compressor cells in the compressor system of the METIS cooler.

\subsection{Performance comparison between the switchless and GGHS configurations}

In order to compare the performance of the METIS cooler with and without GGHS, the dynamic model was adapted to evaluate the dynamic performance of the GGHS configuration as well. A comparison was made by calculating the required input power and number of the cells for the METIS cooler chain for both cases, and the results are shown in Figure 5.10. In this calculation, the carbon diameter was fixed at $14.5 \mathrm{~mm}$ whereas the container wall thickness was automatically adapted to the container diameter and the high pressure. The performances of a single cell are listed in Table 5.1. For the helium-stage compressor that is operating with a relatively small pressure ratio, the switchless configuration can produce $31.8 \%$ more flow rate with $51.7 \%$ additional input power compared to the GGHS configuration. In the hydrogen stage, the pressure ratios are up to 14.6 and 13.2 for the $1^{\text {st }}$ stage and $2^{\text {nd }}$ stage of the compressors, respectively. To achieve such high pressure ratios, the adsorbent has to be heated up to $135.3 \mathrm{~K}$ and $166.0 \mathrm{~K}$, respectively. However, the heating power for the switchless compressor is limited at $150 \mathrm{~W}$ resulting a long heating time during which a large part of the heat is lost via conduction. Consequently, the flow rates that are generated by the switchless hydrogen compressors are only slightly higher but at much higher input powers. Similarly, the switchless neon com-

Table 5.1. Single cell performance comparison between the switchless and GGHS configurations.

\begin{tabular}{|c|c|c|c|c|}
\hline \multirow{2}{*}{ Stage in METIS cooler } & \multicolumn{2}{|c|}{ Input power $(\mathrm{W})$} & \multicolumn{2}{|c|}{ Flow rage $(\mathrm{mg} / \mathrm{s})$} \\
\hline & Switchless & GGHS & Switchless & GGHS \\
\hline Helium & 12.640 & 8.331 & 2.559 & 1.942 \\
\hline Hydrogen, $1^{\text {st }}$ & 13.145 & 9.801 & 1.154 & 1.144 \\
\hline Hydrogen, $2^{\text {nd }}$ & 23.240 & 14.700 & 1.496 & 1.465 \\
\hline Neon & 12.679 & 12.483 & 9.856 & 10.111 \\
\hline
\end{tabular}




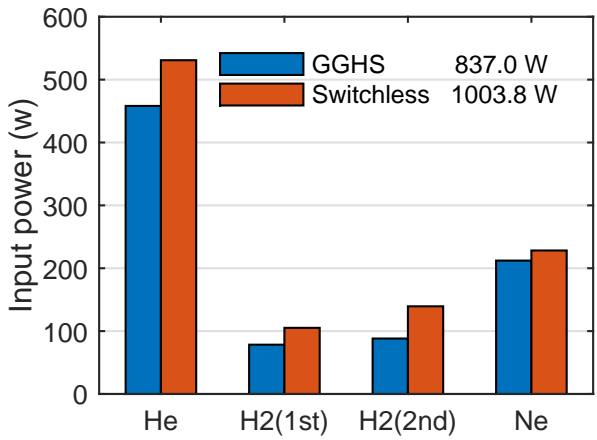

(a)

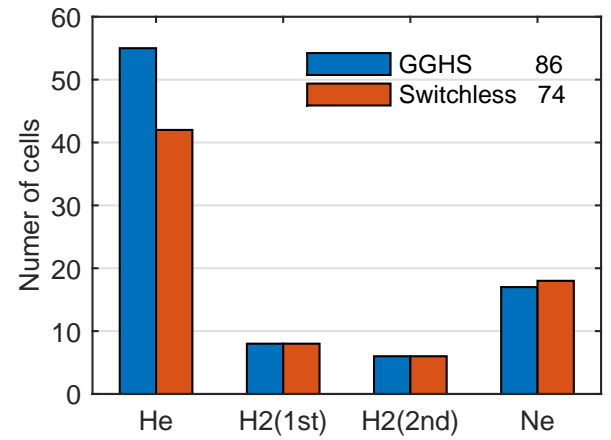

(b)

Figure 5.10. Input power (a) and cell number (b) comparison, GGHS configuration (blue bars) versus switchless configuration (red bars).

pressor has the same disadvantage of high conduction loss. However, the high pressure of the neon compressor is above 90 bar leading to a thick container design in the GGHS configuration. Therefore, the efficiency of the GGHS neon compressor is compromised by the fact that considerable heat is wasted in heating the container wall. As a result, the two cases for the neon compressor perform almost equally well.

Furthermore, the input power and the required number of cells for the METIS cooler chain were calculated based on the single cell performance. As illustrated in Figure 5.10, a major reduction in the number of cells is realized by the switchless configuration in the helium compressor. In total, by using the switchless configuration the METIS cooler requires $14.0 \%$ fewer cells, at the cost of $19.9 \%$ more input power compared to the GGHS configuration.

In the above analysis the performance evaluation for the GGHS configuration is quite optimistic since the GGHS behavior in the model is simplified. The GGHS is simulated by changing the effective conductivity of the contact gas in the gas-gap. Therefore, the dynamic behavior of the GGHS is not included. In a realistic GGHS, however, the switching between "ON" and "OFF" states takes time. For instance, in the hydrogen compressor developed in the previous project, it took $35 \mathrm{~s}$ for the GGHS to switch from "ON" to "OFF" and $10 \mathrm{~s}$ to switch back. Including the dynamics of the GGHS will make the cycle time longer and result in more parasitic conduction loss. In addition, the input power needed to actuate the GGHS is not included in the model, which can be about 2-3\% according to previous experience.

If the sorption compressors in the METIS cooler were to use the GGHS, as mentioned in Chapter 3, then hydrogen actuated by a metal-hydride would be a suitable contact gas. The operating temperature would be at room temperature requiring a long GGHS tube to minimize the conduction loss. Consequently, the switching time could be quite long and the actuating power combined with the conduction loss via the GGHS tube will contribute significantly. We estimate that the performance of the GGHS compressors may be $10 \%$ 
Table 5.2. Dimensions of the switchless sorption compressor for the METIS cooler.

\begin{tabular}{lccccc}
\hline \multirow{2}{*}{ Dimensions } & \multirow{2}{*}{ Unit } & \multirow{2}{*}{ Neon stage } & \multicolumn{2}{c}{ Hydrogen stage } & \multirow{2}{*}{$1^{\text {st }}$ stage } \\
$2^{\text {nd }}$ stage & Helium stage \\
\hline Container wall thickness & $\mathrm{mm}$ & 1.30 & 0.40 & 0.40 & 0.25 \\
Insulation thickness & $\mathrm{mm}$ & 2 & 1.5 & 2 & 1.25 \\
Material of the insulation & - & Kapton & Kapton & Kapton & Kapton \\
Diameter of the adsorbent & $\mathrm{mm}$ & 14.5 & 14.5 & 14.5 & 14.5 \\
Length of the cell & $\mathrm{cm}$ & 50 & 50 & 50 & 50 \\
\hline
\end{tabular}

worse and the required number of cells will be $5 \%$ more if the dynamics of the GGHS behavior is included.

In conclusion, combining the many advantages of the switchless configuration and the limitations of the GGHS, it is worth to sacrifice $20 \%$ input (actually less than that) to gain great simplicity in design, fabrication and operation of the sorption compressors in the METIS cooler.

\subsection{Detailed design of the sorption compressor cells for the METIS cooler}

By starting from the optimized operating conditions discussed in Chapter 3, the dimensions of the switchless sorption compressor cells for the METIS cooler were optimized. As shown in the system level design presented in Chapter 3, the helium stage compressor is the dominant stage which accounts for $53 \%$ of the input and $60 \%$ of the number of cells in the total sorption compressor system for the METIS cooler. Therefore, the helium-stage compressor was optimized first. Then the same carbon diameter was chosen for both the neon and hydrogen compressors to reduce the cost. The same optimization processes were carried out for the neon and hydrogen compressors. The dimensions of the switchless sorption compressor cells for the METIS cooler are presented in Table 5.2. For the METIS cooler 74 cells are required in total, that are $42(56.8 \%)$ cells for the helium stage, $8(10.8 \%)$ and $6(8.1 \%)$ cells for the $1^{\text {st }}$ and $2^{\text {nd }}$ stages of the hydrogen stage, respectively, and $18(24.3 \%)$ cells for the neon stage. The calculated total input power is $1004 \mathrm{~W}$ with $531(52.9 \%) \mathrm{W}$ for the helium stage, $245(24.4 \%) \mathrm{W}$ in total for the hydrogen stage and $228(22.7 \%) \mathrm{W}$ for the neon stage.

\subsection{Conclusion}

In this chapter, a switchless sorption compressor configuration is proposed for the METIS cooler. A dynamic model was built and experimentally verified for simulating this new configuration and for predicting the performance of the sorption compressor. Compared 
to the original configuration with a GGHS, the switchless design requires $20 \%$ more input power but $14 \%$ fewer cells, and it greatly simplifies manufacturing and it reduces production costs. Based on the model the detailed dimensions of the switchless compressor cells for the METIS cooler were determined. 


\section{Scaled Helium Sorption Compressor}

In this chapter, the design of the helium sorption compressor for the METIS cooler is validated in an experimental setup with a down-scaled version of this compressor. The setup is first described on the system level to create an overview of the setup, to nail down the goals of this experiment and to introduce the detailed design of the various components. According to the optimization results obtained in Chapter 5 by using the 1dimensional dynamic sorption compressor model, the sorption compressor cell assembly was designed. The detailed design of the critical components in the test setup such as the heat sink, the check valves, the buffers and the heat exchanger are discussed.

Next, experimental procedures and results are discussed, starting with the preparation and the set-up cool-down process. Introductory test experiments showed a malfunctioning of the check valves at cryogenic temperatures. The solution to this problem is discussed and extensive experiments were performed including single-cell operation, multi-cell operation, and verifying the effects of the aluminum foil inserts, the cycle time and the heat-sink temperature on the performance of the compressor. 


\subsection{Overview of the experimental setup}

As a second demonstrator, the helium sorption compressor setup was made for verifying the design and performance of the most critical stage in the METIS sorption compressor system. In the design of the METIS helium compressor, 46 cells are used. However, the parallel operation of these cells allows us to verify the design and performance in a down-scaled version using only a limited number of cells.

The setup was designed for four full-size helium compressor cells, expected to deliver $8.3 \%$ of the total mass flow rate (i.e. $8.90 \mathrm{mg} / \mathrm{s}$ ) required for the METIS helium stage. However, the single-cell operation should also be tested. The experimental data should be compared to the simulation results for validating the 1-dimensional dynamic model discussed in Chapter 5. Analyzes were done for improving the cell performance by reducing the radial temperature gradient in the carbon. The results indicate that the helium compressor cell can gain $24 \%$ more mass flow by adding aluminum foils between the carbon pills, without degrading the efficiency [84]. This concept was also tested in the experimental setup. Furthermore, the compressor performance against the cycle time and the heat-sink temperature is also investigated.

Figure 6.1 presents the schematic layout of the down-scaled helium sorption compressor setup. The manufacturing of the sorption compressor cells was started with six cells (number 1-6), and four of these were selected (cells 1, 3, 4, and 5). These were mounted in the setup in parallel with individual check valve sets. Cells 1 and 3 are baseline cells (BSC) with no aluminum foils between the carbon pills, whereas Cells 4 and 5 are radial conductance enhanced cells (RCEC) in which aluminum foils with a thickness of $40 \mu \mathrm{m}$ and a diameter of $12 \mathrm{~mm}$ are placed between the carbon pills.

The $70 \mathrm{~K}$ heat sink in this setup is provided by a GM cooler. The four cells surround the cold head of the GM cooler and are directly mounted on the $1^{\text {st }}$ stage cold plate. The cells are also linked to the $2^{\text {nd }}$ stage cold plate by means of thermal straps. The heatsinking is discussed in more detail in Sec. 6.2.2.

Several buffers are connected in series to smoothen the pulsed out-flow generated by the compressor cells and to reduce the storage pressure of the system at room temperature (discussed in Sec. 6.2.4). The buffers, as well as the $70 \mathrm{~K}$ radiation shield, are thermally attached to the $1^{\text {st }}$ stage of the GM cooler. The cold pressurized flow is warmed up to room temperature through a CFHX by the returning flow and then measured by the mass flow controller M2. The pressures at both sides of M2 are measured. The opening of M2 is controlled to maintain the average high pressure at a constant of 14.08 bar for the METIS helium cooler according to the optimized design presented in Table 3.5. For this purpose, the flow controller M2 is set by software to have a mass flow that is proportional to the measured high pressure $p_{h}$, thus establishing $\dot{m}=C p_{h}$. At the start of the compressor operation there is no pressure built up yet, and $C$ is set to zero, i.e. the valve in M2 is fully closed. When the high pressure has reached a certain threshold level (e.g. 13.50 bar), $C$ is set to an initial value that is based on the simulation results. This setting of $C$ determines 


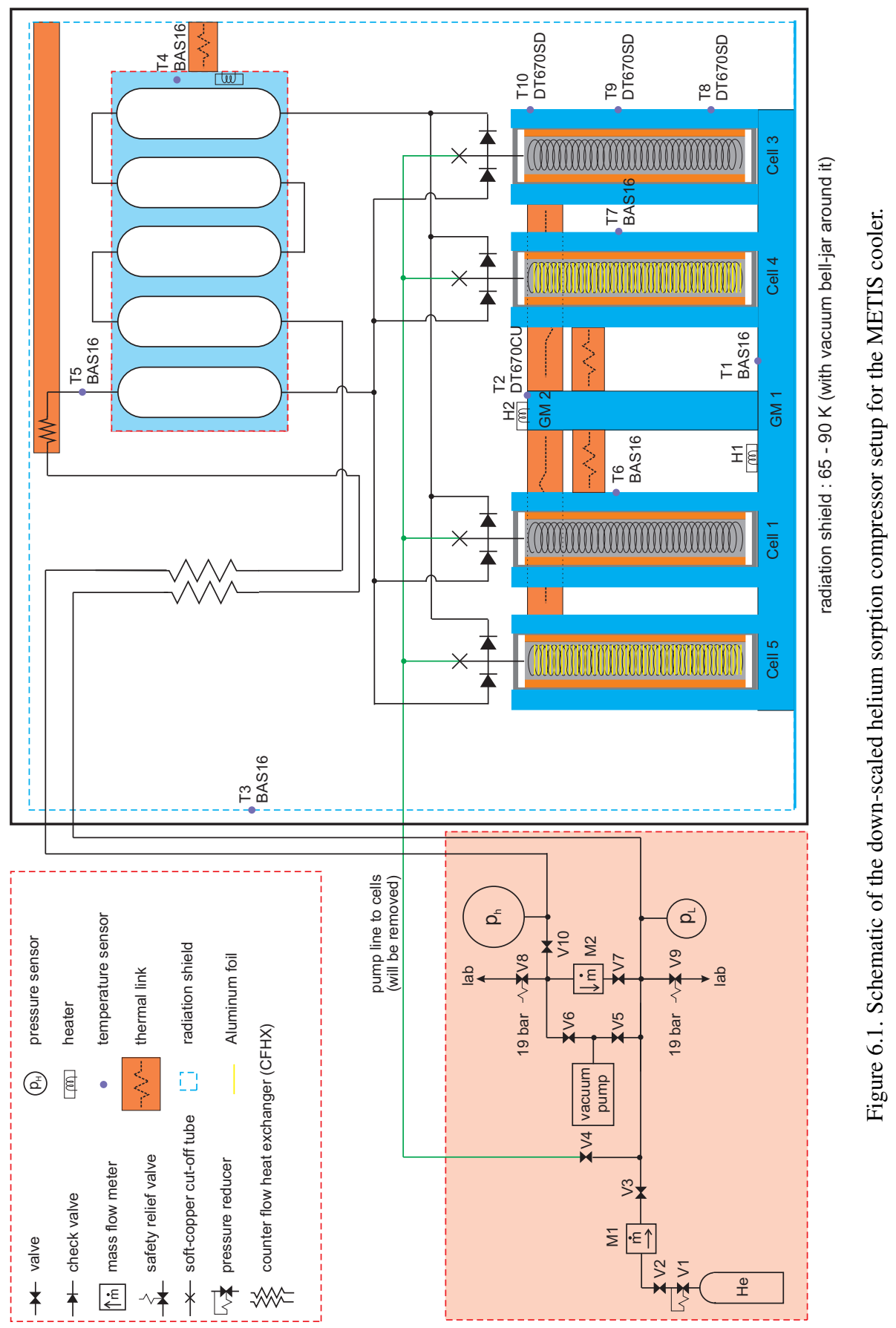


the high pressure. If the average high pressure is higher than the intended value (in this case 14.08 bar), then the value of $C$ in the control should be increased (the valve in the controller will open a bit more). Obviously, if the average pressure is too low, then the value of $C$ needs to be decreased. This iteration repeats until the average pressure is at the intended level. The value of $C$ at that point gives the corresponding mass-flow rate. The low pressure should be 7.62 bar and is determined by M2 and V7, and by the filling pressure and the operating conditions of the system. Therefore, an additional mass flow meter M1 is used to record the mass that is filled into the system.

In order to have the temperature of the in-flow into the compressor cell at $70 \mathrm{~K}$, an additional heat exchanger is added between the low-pressure buffer and the CFHX. Soft copper pinch-off tubes connect the cells to the vacuum pump allowing to fill, pump and flush the cells before the experiment. After that, they are be pinched and sealed, and the pumping line (green line) is removed.

\subsection{Detailed design}

\subsubsection{Sorption compressor cell}

Figure 6.2 is a drawing of the helium sorption compressor that presents the detailed design of the cell. The cell dimensions followed the optimization results listed in Table5.2 for the helium stage, except for the thickness of the container wall that is much thicker ( $1.5 \mathrm{~mm}$ instead of $0.25 \mathrm{~mm}$ ). Using a thicker wall will not degrade the helium compressor performance too much because the temperature swing and gradient in the container wall will be very limited compared to that in the carbon and the insulation layer, as shown in Figure 5.3 (e). On the other hand, this thicker wall allows us also to operate the cells with neon for test purposes if need at very high pressures (up to 100 bar).

The cell container was sealed by a blind cap (the cap in detail B) at one side (blind side) and a similar cap (the cap detail $\mathrm{C}$ ) with the sealed heater and check valves set at the other side (open side). The end caps were designed not just for standing high pressure but also for orbital welding. FEM thermal analysis was performed to optimize the cap dimensions such that parts in the container are not burned during the welding process.

The adsorbent material is saran carbon. It has a porosity of $50 \%$ and was shaped into cylindrical pills with a height of $5.08 \mathrm{~mm}$ and a diameter of $14.5 \mathrm{~mm}$. A central hole of $1.15 \mathrm{~mm}$ was drilled for the heater. The space for the carbon in the container is $505 \mathrm{~mm}$ long. Therefore, 99 carbon pills were used for filling the baseline cell and 98 pills for the radial conductance enhanced cell (incorporating $\mathrm{Al}$ foil). The insulation layer between the carbon and the container wall was made by wrapping Kapton foil (500FN131, thickness of $127 \mu \mathrm{m}$ ) into an annulus cylinder. The cryogenic shrinkage of the insulation layer will be about $3.8 \%$ (data for Polyamide from Ref. [85]) which is taken into account in the parasitic volume and corrected for in the model. The gas channels created by the Kapton shrinkage are negligibly small. However, the triangular channel formed between 


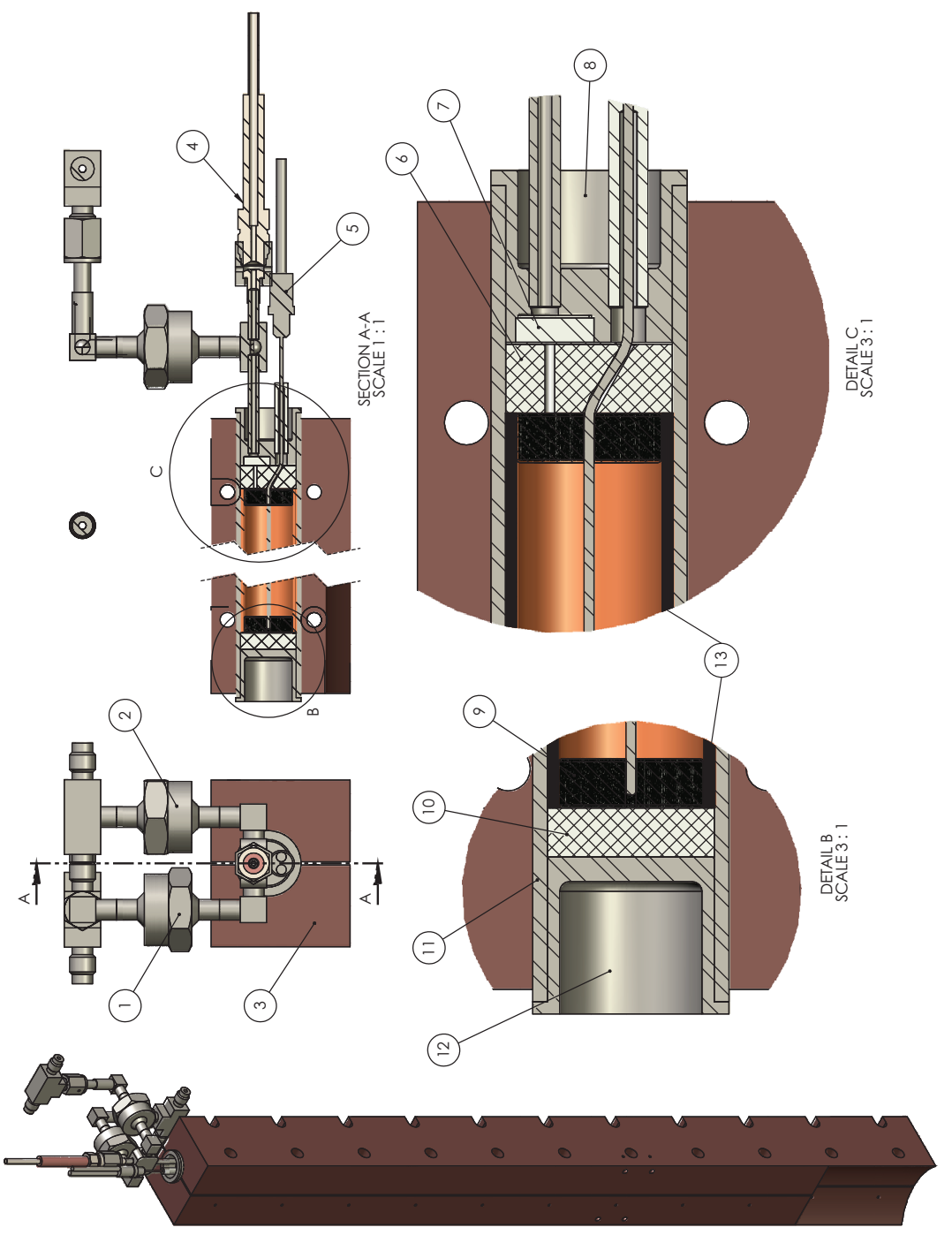

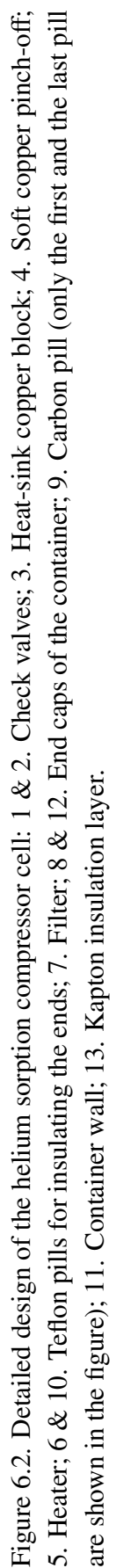


the Kapton film edge and the carbon pills in the axial direction, with an approximate hydraulic diameter of the film thickness, naturally becomes the channel for the working fluid flowing in and out. The thermal insulation between cell and heat sink at the ends of the cell was established by two Teflon pills (details B and C in Figure 6.2). A sintered stainless steel filter with media grade $100 \mu \mathrm{m}$ and a thickness of 0.1 inch (Mott 1100-44-0.1-100) was placed between the Teflon pill and the gas tube connection to the check valves, for preventing any carbon particles to flow into the JT cold stage which could cause clogging (part 7 in detail $\mathrm{C}$ ).

\subsubsection{Heat sink}

As mentioned in Chapter 3, the performance of the helium sorption compressor is very sensitive to the heat-sink temperature. For the real METIS cooler, the heat sinking will be provided by a pumped liquid-nitrogen bath that can easily achieve a quite uniform and steady heat-sink temperature. However, in the test setup, the heat sinking is provided by a GM cooler. It raises the question how to create a uniform and steady heat-sink temperature for dissipating the heat that is generated in four half-meter long cells with the limited cooling power of the GM cooler, and the limited space available in the vacuum bell jar.

The initial design of the heat sink is using thick copper blocks for clamping the cells, as shown in Figure 6.2. These copper blocks were to be mounted on the $1^{\text {st }}$ stage of the GM cooler with a good thermal contact. Furthermore, thermal straps were planned between the middle of the copper blocks and the $2^{\text {nd }}$ stage of the GM cooler. Thus, the heat of each cell flows to the two stages of the GM cooler via two thermal links. A relevant parameter in this configuration is the temperature profile along the compressor cell that obviously should be as small as possible (because of the strong dependence of performance on sink temperature). Furthermore, due to the cyclic operation of the cells the heat flow is varying in time and, therefore, the heat-sink temperature will not be stable in time.

An FEM thermal analysis was performed in SolidWorks to have a first impression on how the heat-sink temperature is influenced by the periodic heat flow from the cell. Here, the heat flowing into the heat sink versus the time was taken from the dynamic cell performance simulation discussed in Chapter 5. This FEM analysis showed that the profile along the cell heat sink is more than $1.3 \mathrm{~K}$, whereas the instability is less than $0.2 \mathrm{~K}$. This instability was considered acceptable for design-validation purposes and, therefore, further detailed design of the heat sinking was focused on reducing the temperature gradient along the cell heat sink.

Figure 6.3 presents the design of the heat-sink assembly and a schematic of the simplified 1-D analysis of the heat conduction along the heat sink. The heat flow from the cell was assumed to be constant, and its density per unit of length equals the average value $P_{L}=25.2 \mathrm{~W} / \mathrm{m}$. The analytical solution of the governing equation for the thermal 


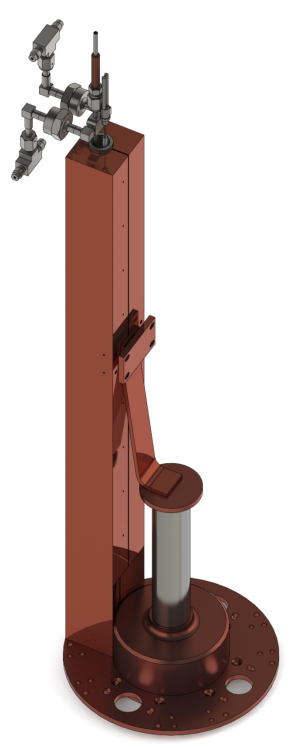

(a)
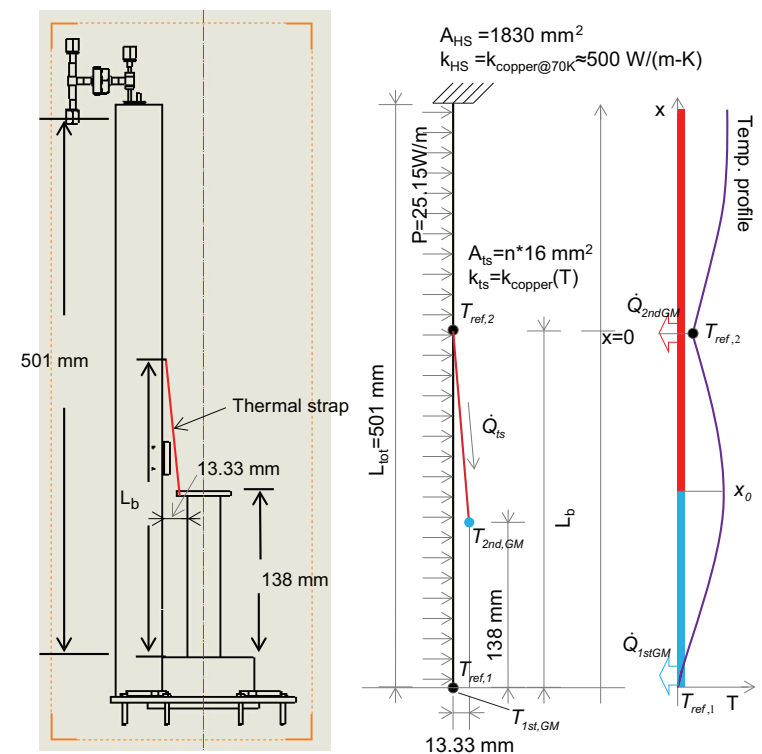

(b)

Figure 6.3. The heat-sink assembly: (a) compressor cell clamped in copper heat sink that is mounted on $1^{\text {st }}$ GM stage plus separate link to $2^{\text {nd }}$ stage; (b) schematic of an 1-D analysis of the heat conduction along the heat sink, and illustration of the parabolic temperature profile along the heat sink.

conduction along the heat sink consists of two parabolic temperature profile segments, as shown in Figure 6.3 (b),

$$
\begin{cases}x \geqslant 0 & T(x)=-\frac{P_{L}}{k_{H S} A_{H S}}\left[\frac{x^{2}}{2}-\left(L_{t o t}-L_{b}\right) x\right]+T_{r e f, 2} \\ x<0 & T(x)=-\frac{P_{L}}{k_{H S} A_{H S}}\left[\frac{x^{2}}{2}-x_{0} x\right]+T_{r e f, 2}\end{cases}
$$

Here, $T_{r e f, 1}$ is the temperature at the interface to the $1^{\text {st }}$ stage of the GM cooler and $T_{r e f, 2}$ is the temperature at the thermal anchoring point of the link to the $2^{\text {nd }}$ stage. $A_{H S}$ and $k_{H S}$ are the cross-sectional area of the heat sink and its thermal conductivity, respectively, whereas $x_{0}$ is the coordinate of the dome of the bottom temperature parabola $\left(x_{0}<0\right)$.

$$
x_{0}=-\frac{L_{b}}{2}+\frac{\left(T_{r e f, 2}-T_{r e f, 1}\right) k_{H S} A_{H S}}{P_{L} L_{b}}
$$

The heat flow from the cell to the sink is split into two streams. The first stream, $\dot{Q}_{1 s t, G M}$, i.e. the heat flow at $x<x_{0}$, indicated by the blue range in Figure $6.3(\mathrm{~b})$, flows to the $1^{\text {st }}$ stage of the GM cooler via the thermal contact at the bottom of the cell. It equals to,

$$
\dot{Q}_{1 s t, G M}=P_{L}\left(L_{b}+x_{0}\right)
$$


The second stream, $\dot{Q}_{2 n d, G M}$, i.e. the heat flow above $x_{0}$, flows to the $2^{\text {nd }}$ stage of the GM cooler via the thermal straps linked to the anchoring point at $x=0$, and can be written as,

$$
\dot{Q}_{2 n d, G M}=P_{L}\left(L_{t o t}-L_{b}-x_{0}\right)
$$

$T_{r e f, 1}$ and $T_{r e f, 2}$ are determined by the heat flows, the contact conductance at the thermal joints and the operating temperatures of the GM cold stages $T_{1 s t, G M}$ and $T_{2 n d, G M}$.

Then the task of reducing the gradient in the heat-sink temperature is to find the combination of the anchor distance $L_{b}$ and the operating temperatures of the GM cooler $T_{1 s t, G M}$ and $T_{2 n d, G M}$ to minimize the difference between the maximum and the minimum temperature along the heat sink. In addition, the cooling capacity of the GM cooler was considered as an important constraint during the optimization. The GM cooler must have sufficient cooling power to compensate the heat flows in Eqs. 6.3 and 6.4.

The heat sink has a cross-sectional area of $1830 \mathrm{~mm}^{2}$ and is assumed to have a thermal conductivity of $500 \mathrm{Wm}^{-1} \mathrm{~K}^{-1}$ (copper at $70 \mathrm{~K}$ ). The thermal link to the $2^{\text {nd }} \mathrm{GM}$ stage was established with copper braiding with a length of $24.2 \mathrm{~cm}$ and a cross-sectional area of $64 \mathrm{~mm}^{2}$. The thermal anchoring point was optimized to be at a distance of $380 \mathrm{~mm}$ from the $1^{\text {st }}$ stage interface. In this case, the calculated temperature difference along the heat sink is $1.05 \mathrm{~K}$.

\subsubsection{Check valves}

Check valves play an important role $[37,70]$ in transforming the periodic pressure swing inside the sorption compressor cell into a more or less continuous flow that drives the JT cold stage. A check valve has to fulfill the following requirements to keep the sorption cooler working properly and efficiently: low void volume, low leak flow, low pressure drop in forward direction, low cracking pressure and high reliability. Furthermore, in the METIS cooler, the check valves have to operate at cryogenic temperature. The combination of these requirements makes most of the commercial check valves not suitable. In a previous project, the University of Twente has developed a space qualified check valve for cryogenic applications [86]. However, to adapt this valve to the specific requirements of the METIS cooler, additional development time would be needed at high costs. Therefore, a Swagelok check valve (6LV-CW4BW6M-NE) with a neoprene sealing material at the valve poppet was selected.

A cross-sectional view of the check valve is shown in Figure 6.4. The forward flow direction is shown by the arrows from the left to the right. This kind of valve is designed for a relatively large flow rate (up to $30 \mathrm{~L} / \mathrm{min}$ ). At a low flow rate (maximum about $0.25 \mathrm{~L} / \mathrm{min}$ for the helium sorption compressor) significant vibrations of the valve were found, resulting in an audible frequency sound noise. That is because small flow cannot push the poppet fully open towards the poppet stop, and that results in an unstable situation. In order to reduce or prevent such unwanted vibrations, the deflection distance of the poppet has to be reduced. The solution was found (and experimentally verified) in 


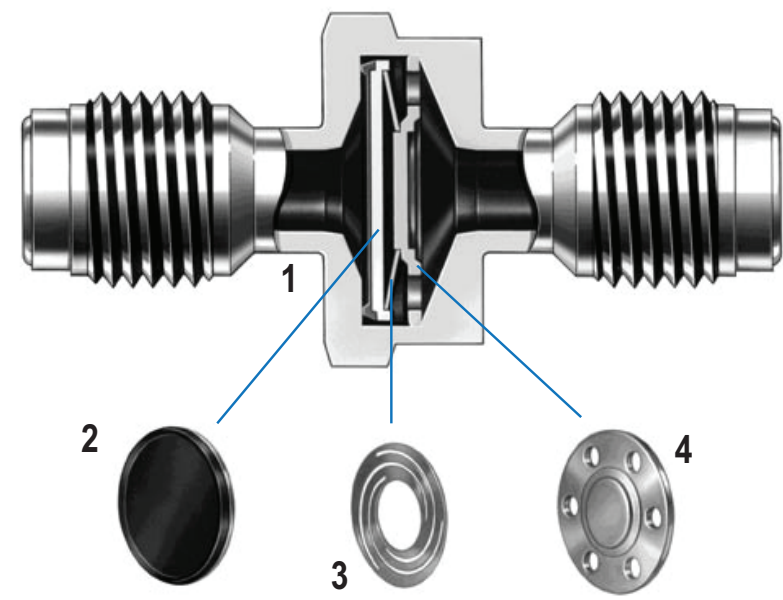

Figure 6.4. Cross-sectional view of the 6LV-CW4BW6M check valve, from Swagelok data sheet (MS-02-89.pdf).

bending the central disc of the poppet stop toward to the poppet by a distance of $0.58 \mathrm{~mm}$ as shown in Figure 6.5.

Sixteen valves were tested at liquid nitrogen temperature ( $77 \mathrm{~K}$ ) to verify leak flow, pressure drop, and cracking pressure. Both the pressure drop and cracking pressure were below 50 mbar, whereas the leak flow rate was below $2 \%$. The void volume per valve is about $2.75 \mathrm{cc}$ that is acceptable for a half meter long cell (the void volume in the porous carbon is up to $41.3 \mathrm{cc}$ ). The ten best performing valves were chosen for the compressorcell assembly.

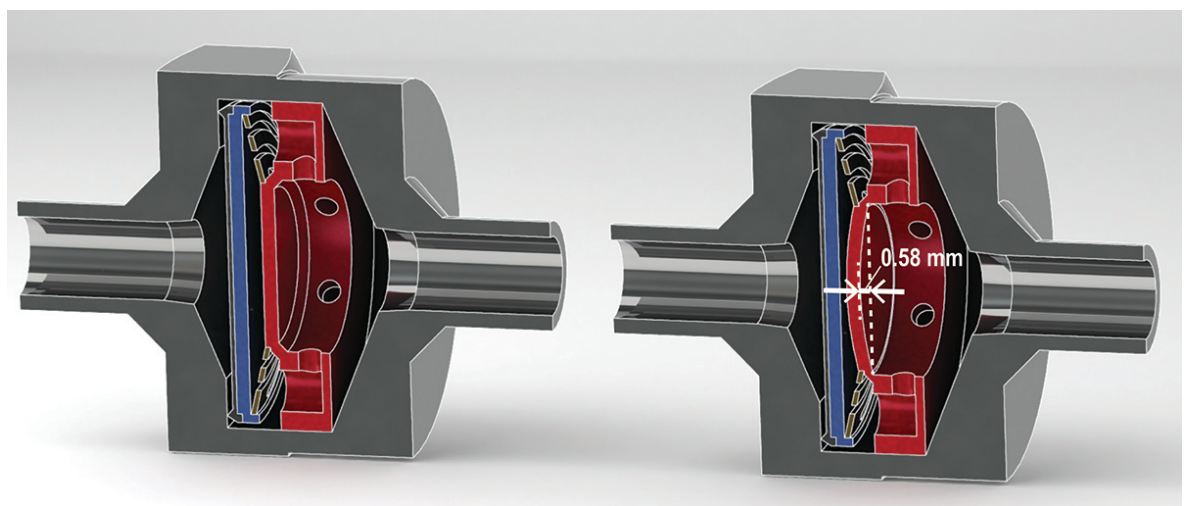

Figure 6.5. CAD drawings to represent the required adjustment to the poppet stop to reducing the unwanted vibration at a low flow rate. 


\subsubsection{Buffers}

Buffers are needed in a sorption compressor system for two reasons: 1. dampening the pressure variation caused by the asynchronous cycling of the sorption cells; 2 . providing a massive volume that reduces the system storage pressure at the room temperature.

Figure 6.6 shows the simulated cyclic operation of the four cells in the test setup, depicting the mass flow rate in and out of the cells. As shown in Figure 6.6, the four cells operate in parallel with subsequent phase differences of $90^{\circ}$. Because of the pulsed heating in the switchless configuration, the flow out of the cells exhibits short, high pulses, which are the positive peaks in Figure 6.6. For the experimental setup described in this chapter, the maximum operating cell number is four so that the peak flows at the highpressure side will cause a significant pressure swing if there is not enough buffer volume. However, for the real METIS cooler, the total cell number is over 40 so that one can cycle them with a much smaller phase difference (less than $9^{\circ}$ ). In that case, the highpressure peak flows largely overlap and consequently the system needs less buffer volume for damping the high-pressure variation. The passive cooling down phase of the cell usually takes a relatively long time that makes the in-flows of the cells small and lasting longer. Therefore, less buffer volume is required for the low-pressure stabilization.

Figure 6.7 shows the simulated pressure swing of the setup with four cells and using buffers of $2.0 \mathrm{~L}$ and $0.5 \mathrm{~L}$ at the high and low-pressure sides, respectively. Here, the mass flow rate in and out of the compressor cell was calculated based on the assumption that the high and low pressures are constant. Figure 6.7 shows that this is a fair assumption since the relative change in pressure is small. The peak-to-peak amplitude of the pressure oscillation depends on the number of cells that are operated, as shown in Figure 6.8. As long as the number of cells is less than 10, the high-pressure out-flows of the cells will not overlap, and the amplitude of the high-pressure oscillation decreases linearly with the number of cells. In the in-flow phase, however, because of the longer peaks, overlap arises for a lower number of cells and the gain in reducing the low-pressure oscillation is quickly "saturated".

The pressures of the system at room-temperature storage for different sizes of the buffers are listed in Table 6.1. The storage pressure increases at larger high-pressure buffer volume as it releases additional dense working fluid. And the storage pressure decreases at larger low-pressure buffer since that provides additional storage volume. Finally $2.0 \mathrm{~L}$ and $0.5 \mathrm{~L}$ for high and low-pressure buffers were chosen. The buffers were made of standard Swagelok sample bottles mounted on the $70 \mathrm{~K}$ platform that is cooled by the $1^{\text {st }}$ stage of the GM cooler.

\subsubsection{Heat exchangers}

The CFHX operating between room temperature and the heat-sink temperature of $70 \mathrm{~K}$ is configured as a tube-in-tube CFHX with a length of $3.0 \mathrm{~m}$ made of $1 / 8 * 0.028$ inch and 


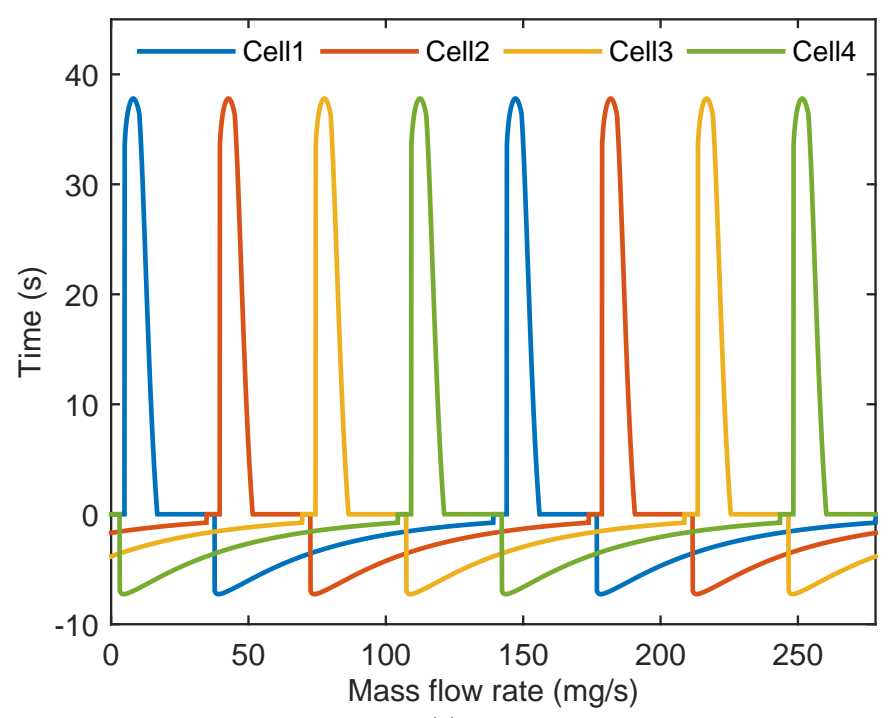

(a)

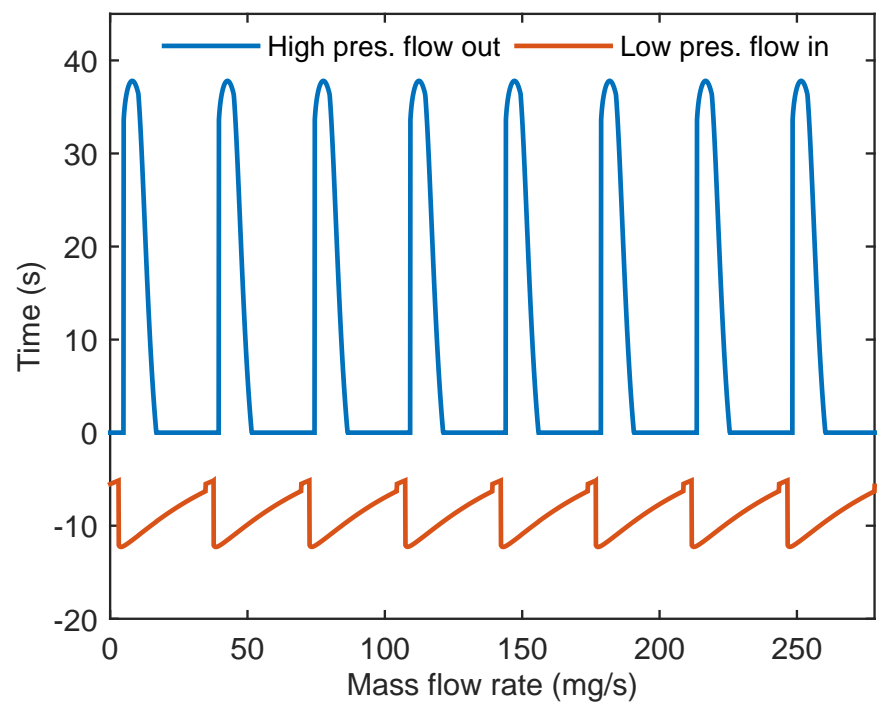

(b)

Figure 6.6. Simulated mass flow rate of the helium sorption compressor cells in the experimental setup. (a) Mass flow rate generated by individual cell; (b) Total mass flow rate generated by 4 cells operating out of phase. 


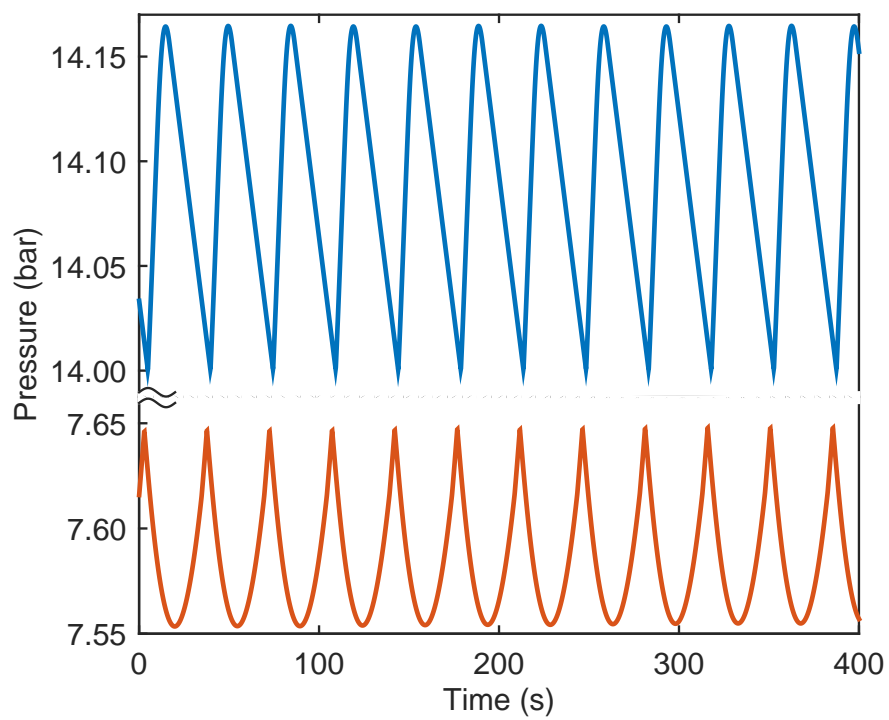

Figure 6.7. Pressure swings in the high and low-pressure sides of the setup with four cells and buffer volumes of $2.0 \mathrm{~L}$ and $0.5 \mathrm{~L}$, respectively.

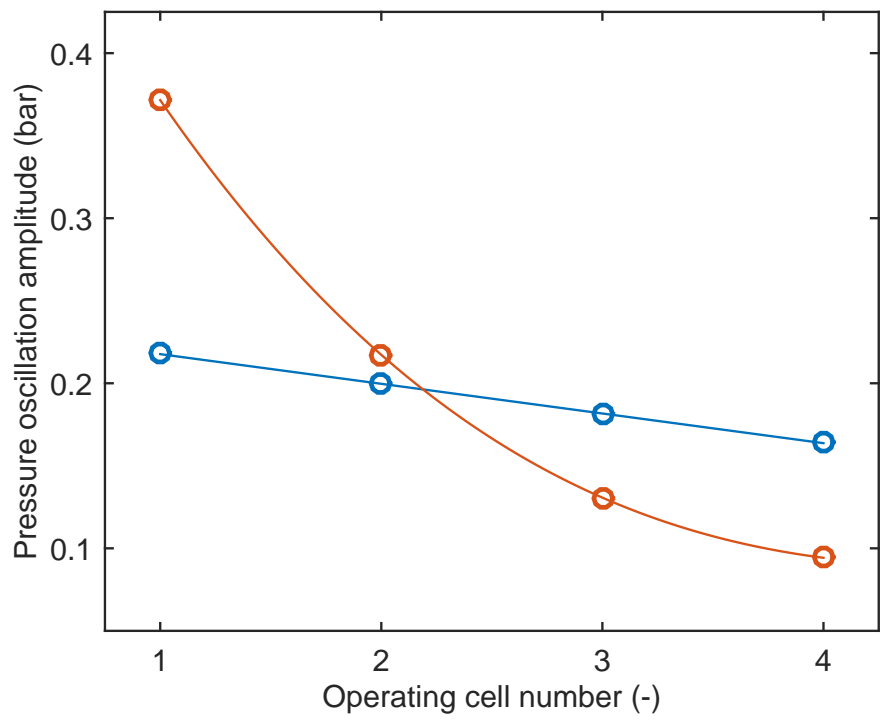

Figure 6.8. Pressure swings in the high and low-pressure sides of the setup as a function of the number of cells, with buffer volumes of $2.0 \mathrm{~L}$ and $0.5 \mathrm{~L}$ for high and low pressure side, respectively. 
Table 6.1. The storage pressure of the system with different sizes of the buffers.

\begin{tabular}{ccc}
\hline High-pres. buffer vol. (L) & Low-pres. buffer vol. (L) & Storage pressure (bar) \\
\hline 2.0 & 1.5 & 50.5 \\
2.0 & 1.0 & 53.5 \\
2.0 & 0.5 & 57.6 \\
1.5 & 1.5 & 48.9 \\
1.5 & 1.0 & 52.1 \\
1.5 & 0.5 & 56.8 \\
\hline
\end{tabular}

$1 / 4 * 0.049$ inch tubing. The inside circular channel is for the high-pressure flow whereas the outside annulus channel is for the low-pressure flow. The estimated effectiveness of the CFHX is over than $99.99 \%$, with pressure drops of 0.011 bar at the high-pressure side and 0.111 bar at the low-pressure side.

The low-pressure in-flow of the compressor cell should be precooled to the heat-sink temperature of $70 \mathrm{~K}$. However, even with a very efficient CFHX, the low-pressure flow could only be cooled to about $83 \mathrm{~K}$ before entering the low-pressure buffer, mainly due to the radiation loss. Furthermore, this evaluation is based on the assumption that the highpressure flow generated by the cell is perfectly precooled to the heat-sink temperature in the high-pressure buffers before it enters the CFHX. Therefore, a $70 \mathrm{~K}$ heat exchanger (HX) is required for precooling the low-pressure flow. This precooling HX was made of a $15 \mathrm{~cm}$ long, $1 / 8 * 0.028$ inch tubing that is thermally attached to the $70 \mathrm{~K}$ platform.

\subsection{Compressor assembling}

\subsubsection{Cell assembling}

Before assembling the compressor cell, the check valves, the gas tube, the gas splitter, the heater and the VCR connectors were welded onto the respective end cap, as shown in Figure 6.9 (a). The sintered stainless steel filter was inserted into the end cap housing between the Teflon pill and the gas-tube opening (see Figure 6.2 detail $\mathrm{C}$ ). The heater was properly bent to pass through the Teflon pill as illustrated in the cross-sectional view in Figure 6.2 (detail C). Figure 6.9 (b) presents the tools, components and material before assembling the sorption compressor cell. The cell container is made of $20 * 1.5 \mathrm{~mm}$ SS316L tube. The inner diameter of the container is $17 \mathrm{~mm}$, and the carbon pill diameter is $14.5 \mathrm{~mm}$ leaving an annular space of $1.25 \mathrm{~mm}$ for the Kapton insulation. The Kapton foil was cut into $0.5 \mathrm{~m}$ wide, and $0.41 \mathrm{~m}$ long. With such size, it can be wrapped into an 8.3 layers annular cylinder with an outer diameter of $17 \mathrm{~mm}$ and a thickness of $1.05 \mathrm{~m}$ $\mathrm{m}$ (theoretically, leaving $0.2 \mathrm{~mm}$ margin). The carbon pills were delivered in a vacuum package that was not opened until the carbon was inserted to prevent any contaminations. 


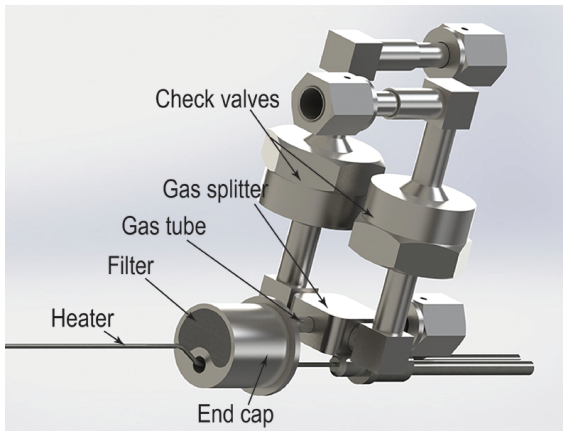

(a)

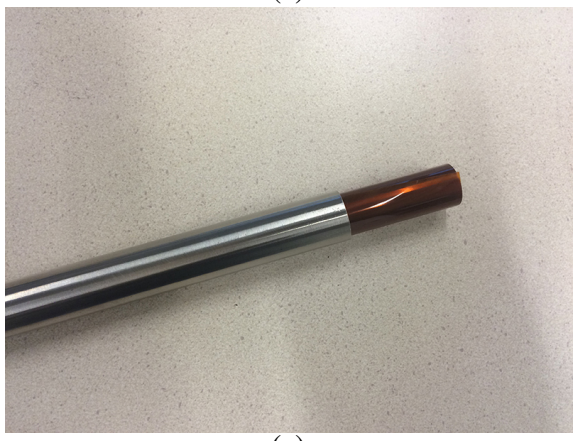

(c)

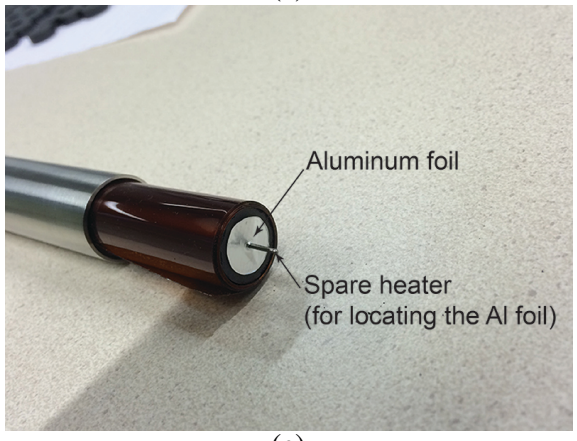

(e)

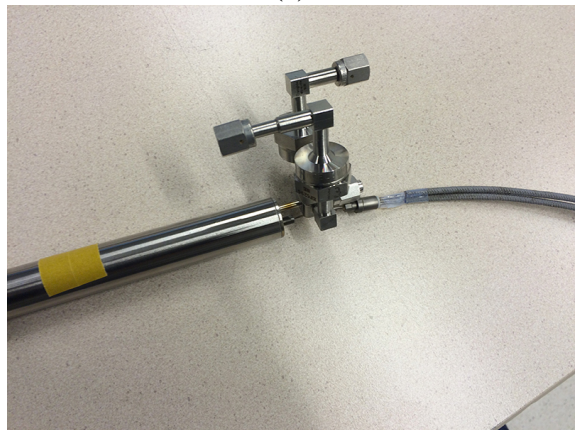

(g)

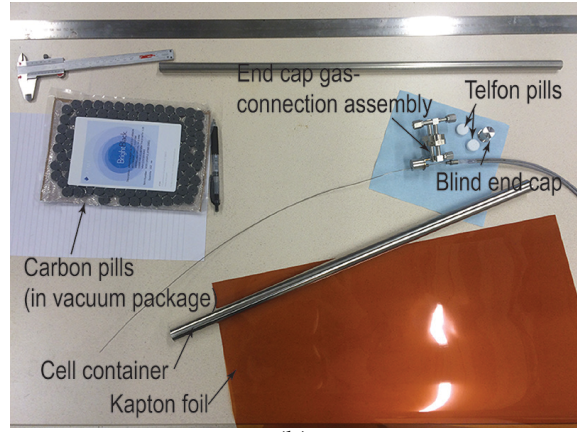

(b)

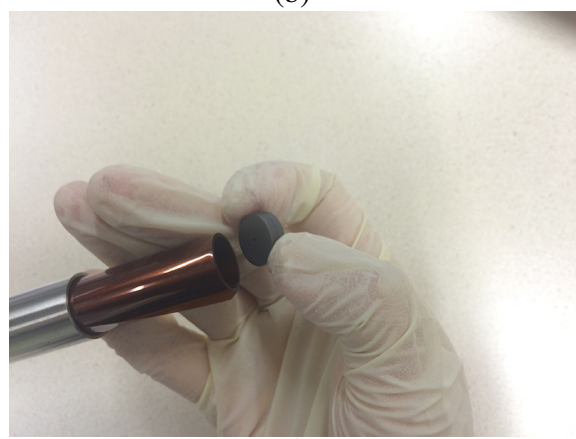

(d)

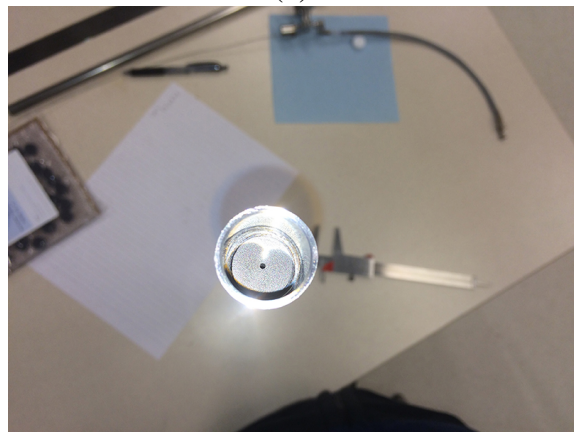

(f)

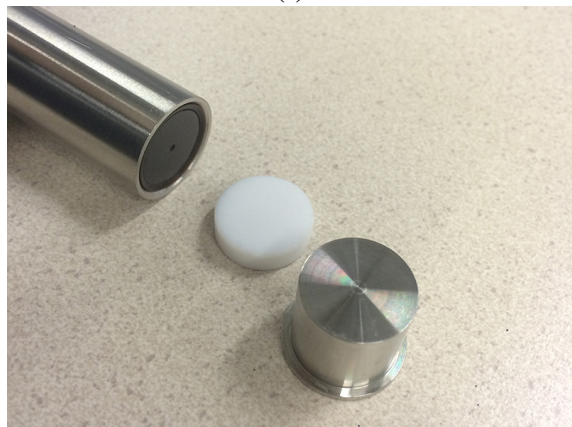

(h)

Figure 6.9. Sorption compressor cell assembling (details explained in text). 
The cells were assembled in the following steps:

1. The Kapton foil was rolled into a cylinder and inserted into the container tube leaving about $5 \mathrm{~cm}$ outside of the tube, as shown in Figure 6.9 (c);

2. Carbon pills were inserted into the Kapton cylinder as shown in Figure 6.9 (d). For the radial conductance enhanced cells, a spare heater was used for positioning the aluminum foils and the carbon pills which are inserted one after the other as shown in Figure 6.9 (e);

3. The Kapton insulation with the carbon pills was pushed into the container tube to its proper position (Figure 6.9 (f)).

4. The heater was inserted through the Teflon pill, then into the central hole in the carbon pills until the end cap closes the container tube (Figure $6.9(\mathrm{~g})$ );

5. The blind side of the container with the Teflon pill and end cap was closed (Figure 6.9 (h));

6. The heater resistance was tested;

7. End caps were welded, and leak testing performed.

Three baseline cells (Cell 1, 2 and 3) and three radial conductance enhanced cells (Cell 4, 5 and 6) were assembled. The heater of Cell 2 appeared to be broken during the welding process. So Cell 2 was discarded for the set-up and used instead for a burst test. It was filled with water up to 160 bar without leakage. Cell 6 had a leak on the check-valve connection. Therefore, Cells 1, 3, 4 and 5 were installed in the set-up.

\subsubsection{Set-up assembling}

The compressor cells were clamped by two copper blocks as the heat sink, as shown in Figure 6.10. Good thermal contact between the cell container and the heat sink was established by using Apiezon $\mathrm{N}$ cryogenic vacuum grease and 22 bolts fixing the construction. Temperature sensors T6 and T7 were mounted in the middle of Cells 1 and 4 (272.5 mm from the bottom of the copper heat sink, corresponding x coordinate of $-144.5 \mathrm{~mm}$ ). T8, T9 and T10 were placed on Cell $3(122.5,422.5$ and $522.5 \mathrm{~mm}$ from the bottom of the copper heat sink, corresponding x coordinate of $-294.5,-5.5$ and $-105.5 \mathrm{~mm}$, respectively), see Figure 6.1.

As shown in Figure 6.11 (a), the aluminum cold plate was first assembled. It was supported by Teflon rods from the bottom of the vacuum bell jar, and thermally linked to the $1^{\text {st }}$ stage of the GM cooler. This cold plate was to support the radiation shield and the buffers. All electronics on the GM cooler such as the heaters $\mathrm{H} 1$ and $\mathrm{H} 2$ and the temperature sensors T1 and T2 were mounted. Thermal straps were also clamped on to the $2^{\text {nd }}$ stage of the GM cooler. Teflon rods were used to mechanically support the $1^{\text {st }}$ stage of the GM cooler from the bottom of the vacuum bell jar in order to carry the weight of the sorption compressor cells.

The second step was to install the compressor-cell assemblies. These were placed through the CFHX that was properly positioned in advance, and the assemblies were 


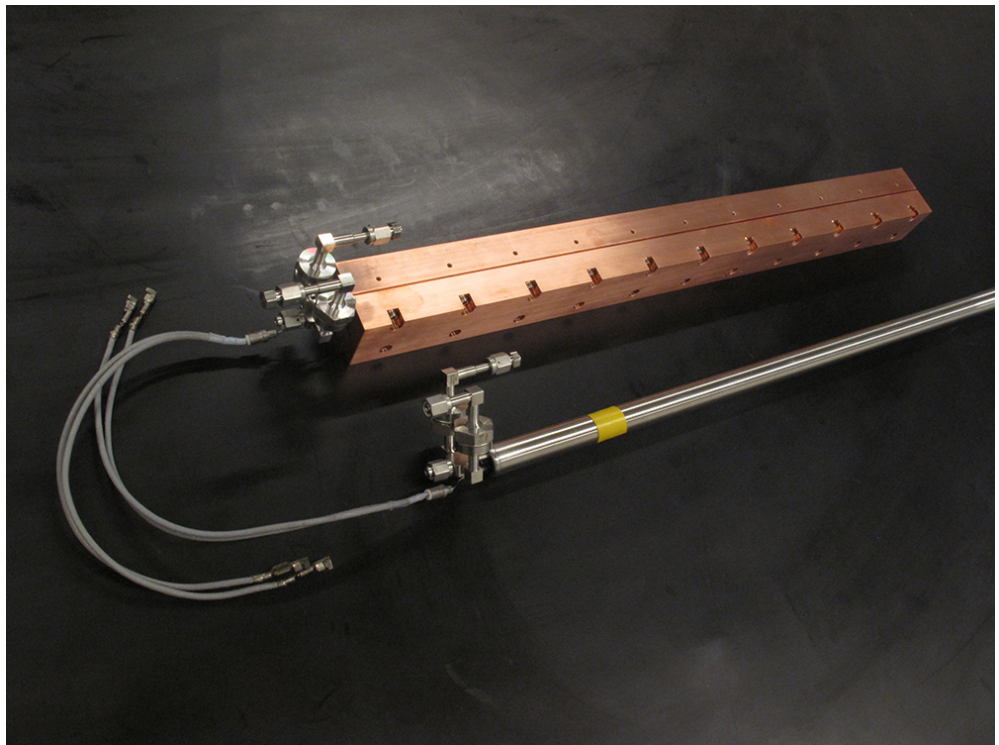

Figure 6.10. Sorption compressor cell assembly with the copper heat-sink blocks.

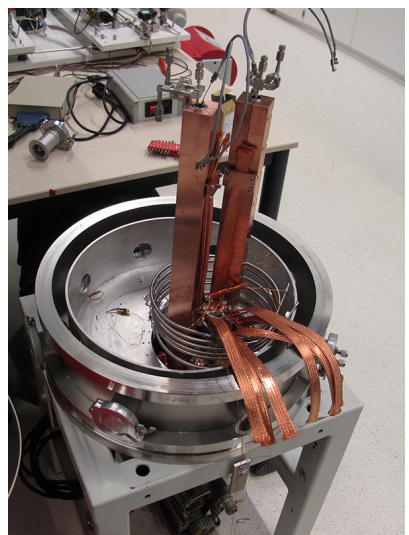

(a)

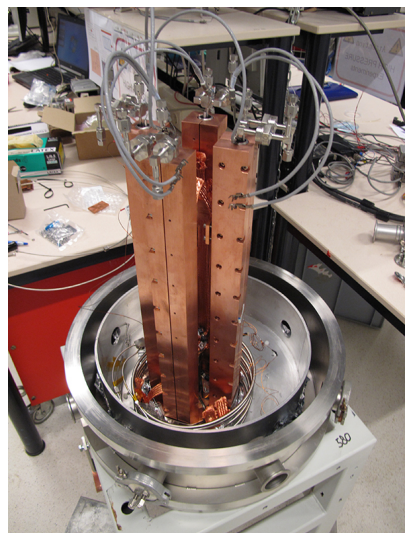

(b)

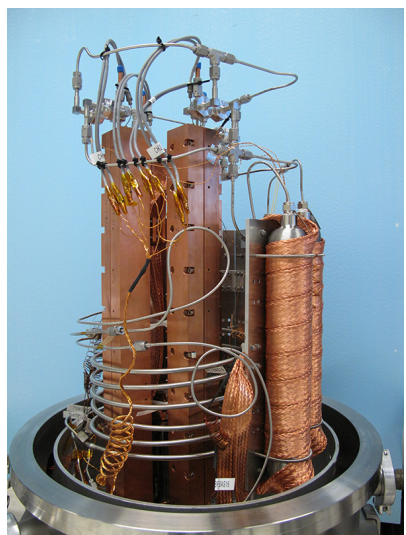

(c)

Figure 6.11. Experimental setup assembly: (a). Two cells plus heat sinks mounted; (b). Four cells mounted; (c). Cells, buffers, CFHX and gas lines installed.

tightly mounted onto the copper plate of the GM $1^{\text {st }}$ stage. Figure 6.11 (b) presents the set-up with all cells mounted.

Next, the buffers were assembled, all tubing was connected properly, and the electronics were installed and tested, as shown in Figure 6.11 (c). Then, the radiation shield was placed, and the vacuum bell jar was closed. Finally, the subsystem outside of the vacuum bell jar was built that includes the pressure sensors, the mass flow controller, the safety relief valves and the interfaces for charging and pumping. 


\subsection{Experiment and discussions}

\subsubsection{Preparation before the cool-down}

During the assembly process, the activated carbon was exposed to air and adsorbed considerable amounts of air and moisture. These would freeze at a cryogenic temperature causing a reduced adsorption capacity and also might cause clogging. Therefore, one of the crucial preparation steps before cooling down the system is cleaning it by flushing, pumping and baking to remove all contamination.

The system was flushed with helium for several times first. Then the compressor cells were baked by the central heater with T6 - T10 (see Figure 6.1) rising to $60{ }^{\circ} \mathrm{C}$ while the central temperature of the cells was about $100{ }^{\circ} \mathrm{C}$. At the same time, the system was pumped down to $10 \mathrm{E}-6$ mbar. The baking process lasted for more than 24 hours. After baking, the system was pre-charged to 5 bar, and a leak test was performed. The integral recording of M1 gave the total amount of helium that was filled into the system.

According to the safety regulation of our lab that maximum $10 \mathrm{bar} * \mathrm{~L}$ is allowed for pressurized vessels, we have to keep the system pressure below 20 bar (considering each buffer bottle is $0.5 \mathrm{~L}$ ). Therefore, the safety relief valve shown in Figure 6.1 is set to 19 bar and the system was further charged at cryogenic temperature since it could not be charged to the storage pressure of 58 bar at room temperature.

\subsubsection{Introductory test experiments}

The system was first cooled down by the GM cooler with M2 and V7 fully open. Thus the pressure in the system was uniform and, during the first 6.5 hours of the cool-down process, it decreased gradually from the initial pre-charging pressure of 5.0 bar to 2.1 bar. Then, the cell temperatures (T6-T10) reached $80 \mathrm{~K}$, and the system was further filled until the total amount of helium in the system reached the required level. The temperatures of the GM cold stages, T1 and T2 were controlled at $60 \mathrm{~K}$ and $50 \mathrm{~K}$, respectively. It took another 9.5 hours to cool the entire cold assembly to these temperatures.

In order to check the system operation, several introductory test experiments were performed. In these experiments, the heater of Cell 1 appeared not to function at the heatsink temperature of $70 \mathrm{~K}$ although it worked fine at room temperature. We were not able to find the exact reason of this failure and to fix it. The most possible reason would be the thermal contraction due to the cryogenic temperature breaking the heater resistor or an internal electrical connection. Since each compressor cell has its own check-valve unit, we were still able to run the other three cells with Cell 1 inactive.

In the individual tests of the other cells (Cells 3, 4 and 5) a serious problem with the check valves appeared. With heat-sink temperature $70 \mathrm{~K}$, peak heating power $150 \mathrm{~W}$, heating time $10 \mathrm{~s}$ and cycle time $60 \mathrm{~s}, \mathrm{M} 2$ and V7 were closed at first and the cells were cycled to build up a pressure difference. As shown in Figure 6.12 (a), for Cells 3 and 4, the high pressure $P_{h}$ (connected to the high-pressure buffer, measured at the outside of the 

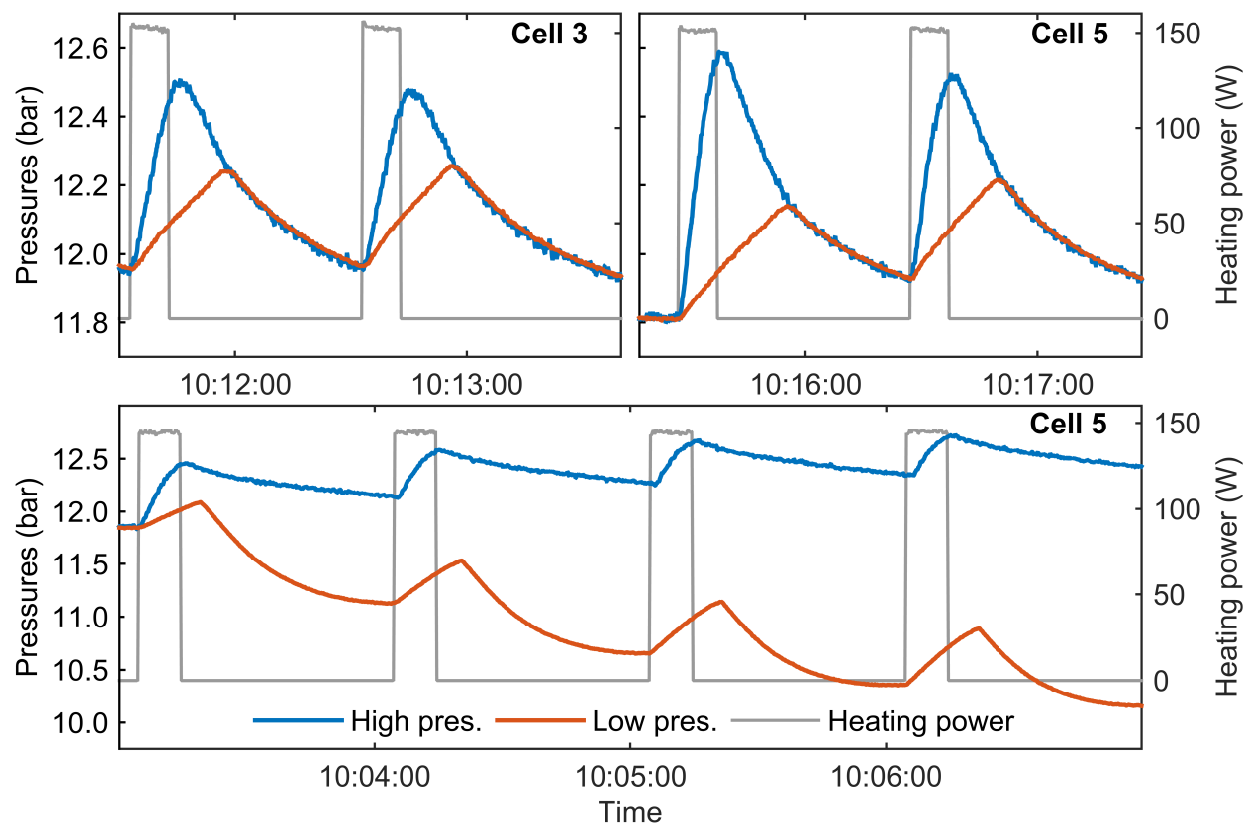

(a)

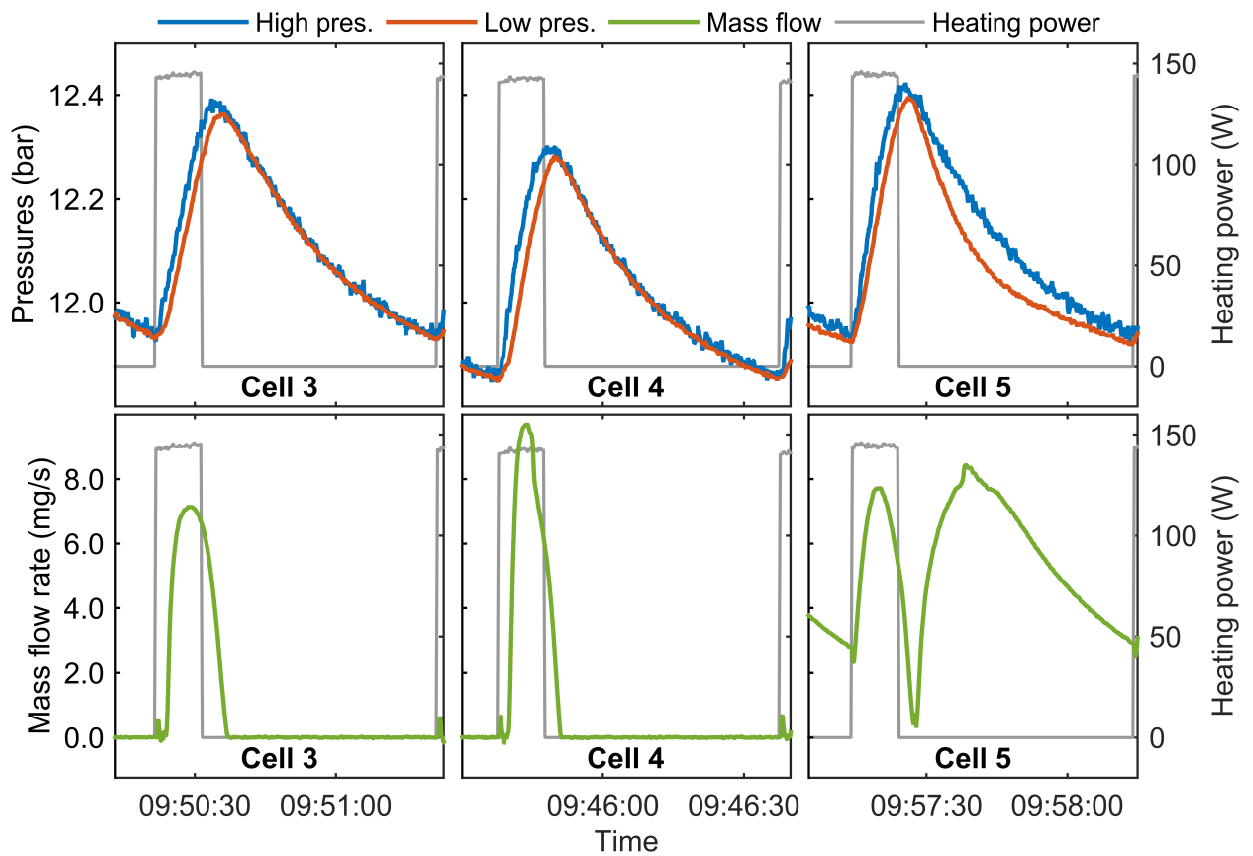

(b)

Figure 6.12. The measured prressures and the mass flow as a function of time in the testing runs. (a) Cycling with M2 closed; (b) Cycling with M2 and V7 fully open. 
system, see Figure 6.1) rose immediately once the heating was applied. This is because the pressures at both sides of the high-pressure check valve initially were equal and it required no time to build up pressure inside the cell. The high-pressure increase continued for a few seconds after the heating had been switched off. Then it dropped quickly which indicated that the high-pressure check valve did not close. The low-pressure check valve also appeared to be open as indicated by the pressure rising at the low-pressure side when the cell was heated. Because of the larger low-pressure buffer volume the low pressure increased slower which created a pressure difference for a while. At about 15-20 s after the heating had been switched off both pressures reduced at the same rate until the next cycle started. Cell 5, however, showed a different behavior. The low pressure rose during the heating process similar to Cells 3 and 4 indicating that the low-pressure check valve was also not closed for Cell 5. But during the cooling process the high pressure did not decrease so rapidly while the low pressure dropped much more which resulted in a pressure difference. This indicates that the high-pressure check valve of Cell 5 can close properly. The decrease in high pressure is due to the fact that the gas enters the buffer at a higher temperature and is cooled down to the heat-sink temperature of $70 \mathrm{~K}$ after the highpressure check valve closes. To further verify the check-valve conditions, a similar test was performed with M2 and V7 fully open thus allowing for a flow from the high-pressure to the low-pressure side of the cells. As shown in Figure 6.12 (b), Cells 3 and 4 generated a peak flow during the heating phase with a small pressure difference between the highand low-pressure sides. Although both the high- and low-pressure check valves were not closing properly, the high pressure responded faster and more than the low pressure because of the buffer size difference and the relatively short heating pulse, that led to a flow peak through M2. The pressures quickly leveled out and dropped simultaneously once the heating was stopped and there was no flow during this phase. For Cell 5, the pressures reached the same level at a few seconds after the heating was off. Meanwhile the mass flow reduced down to a minimum. Then, both pressures decreased. But the low pressure dropped faster resulting in an increase in mass flow through M2. This only could happen if the high-pressure check valve was closed during the cooling phase.

As the most probable reason causing the check-valve failure, we considered the cryogenic operating temperature although their operation was verified at $77 \mathrm{~K}$ as mentioned in Sec. 6.2.3. A small effect may have been caused by the fact that the valves in the compressor setup were actually cooled down to about $60 \mathrm{~K}$ before controlling the heat-sink temperature to $70 \mathrm{~K}$. The extra cooling may have caused the failure. However, a larger effect is expected from the fact that the check valves were cooled down without any pressure difference between the two sides. The check valves need a certain pressure difference that presses and deforms the sealing material to create a leak-tight sealing (neoprene for these valves, see Figure 6.4). Cooling down without a pressure difference kept the sealing material in its original shape. Apparently, the sealing material lost most of its elasticity at the cryogenic operating temperature and could not deform enough to establish a tight seal. 
To tackle these effects, heaters and temperature sensors were placed on the checkvalve units to keep them at the proper operating temperature. In addition, we kept a certain pressure difference between the check valves $\left(p_{h}>p_{\text {cell }}>p_{l}\right)$ during the cooldown process and the filling procedure to keep the sealing material in a deformed state. The further experiments discussed in Sec. 6.4.3 showed that this approach worked well and the leak rate of the check valves was sufficiently low.

\subsubsection{Single-cell experimental results}

A default setting of each cell was chosen according to the simulation results of the sorption compressor model: pulsed heating power for each cell $150 \mathrm{~W}$, heating time $8.73 \mathrm{~s}$, cycle time $103.6 \mathrm{~s}$, average heat-sink temperature $70 \mathrm{~K}$ and high pressure was 14.08 bar. Although the buffer volumes were used in the system to dampen the pressure swing, there was still significant pressure oscillation along the cycling period, especially for single-cell operation. As explained in Sec. 6.1, M2 controls the mass flow in proportion to the average high pressure $\left(\dot{m}=C p_{h}\right)$. In the case of single-cell operation the average is taken over each single full cycle. The value of $C$ is stepwise changed until the measured average pressure equals 14.08 bar. In this process, the ratio of the new value of $C$ to its old value equals the ratio of the measured average high pressure to the desired value (i.e. 14.08 bar). So, if the pressure is a factor of two too high, then $C$ should be increased by that factor of 2. After a few cycles the average pressure is 14.08 bar and the value of $C$ at that point gives the corresponding mass-flow rate.

The performance of the individual cells operating at nominal conditions was tested. In Figure 6.13, the plots on the left present the pressure, mass flow rates (raw and filtered) and temperature measurements of Cell 3 operating at nominal conditions. The pressure swings in high and low pressures were about 0.4 bar. These pressure swings influence the performance of the sorption compressor since they allow the cell to produce an outflow at relatively higher pressure and to adsorb an in-flow at relatively lower pressure. As explained above, the average high pressure was controlled at 14.08 bar. The measured average low pressure was $7.48(7.48 \pm 0.05)$ bar. As a result of the dynamic control in M2, the mass flow showed significant high-frequency noise. This was filtered by a SavitzkyGolay filter ( $k=3, f=399, k$ is the polynomial order and $f$ is the frame size), resulting in a flow fluctuation of less than 15\%. At the high pressure of 14.08 bar, Cell 3 established an average mass flow rate of $2.73(2.73 \pm 0.05) \mathrm{mg} / \mathrm{s}$. The heat-sink temperature oscillated about $0.3 \mathrm{~K}$ due to the periodic heating. According to T8, T9, T10 and the cooling temperatures of the GM cooler T1 and T2, the average heat-sink temperature of the copper heat-sink blocks is $70.6(70.6 \pm 0.5) \mathrm{K}$. And the maximum temperature deviation of the heat-sink temperature is about $0.9 \mathrm{~K}$ which is very close to the estimation of $1.05 \mathrm{~K}$ in Sec. 6.2.2.

The right plots in Figure 6.13 zoom in on one particular cycle. In the heating period, the high pressure did not rise until the pressure in the cell was built up in about $4 \mathrm{~s}$. Then 
(M) дәMod 6u!̣eән
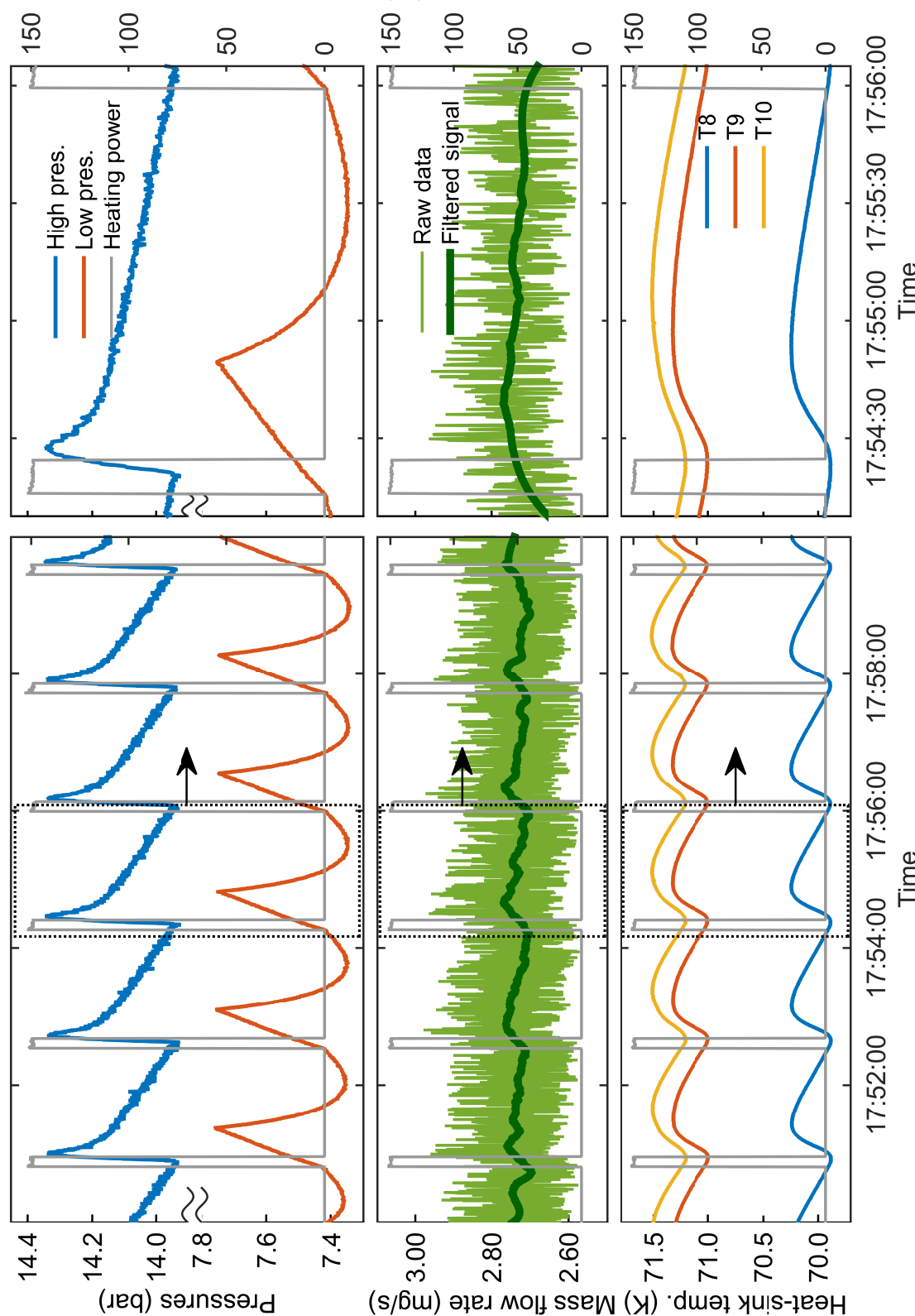

(2)

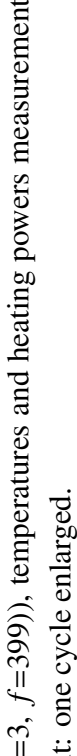

II

¿

유

○ं

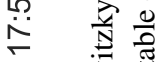

실

8 क

i் ते

豆

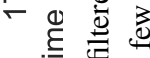

$\mathrm{O}^{i}$

ষ்

然是

할

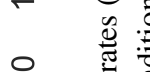

일

$\stackrel{1}{\sim}$

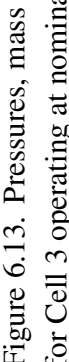




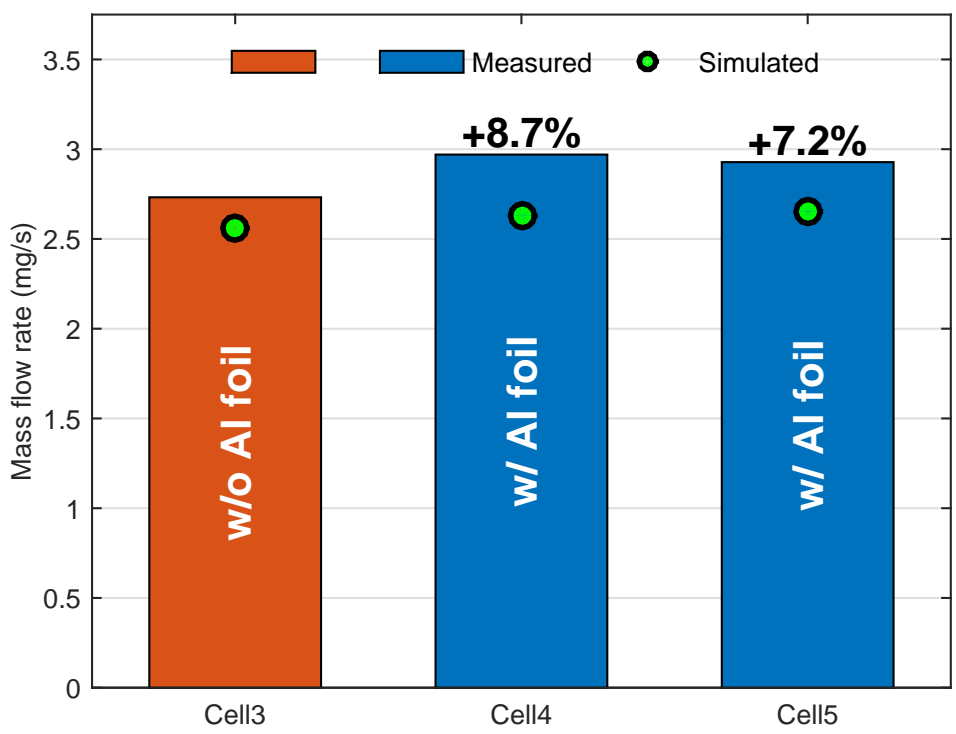

Figure 6.14. Measured average mass flow rate comparison between Cells 3, 4 and 5.

the high-pressure check valve opened and the high pressure increased rapidly. Meanwhile, the low pressure rose linearly because of the mass flow from high-pressure side to the lowpressure side. When the heating stopped, the high-pressure check valve closed in about $3.5 \mathrm{~s}$. Then the high pressure started to decrease because, firstly, the flow continued out of the high-pressure buffer and, secondly, the pressurized hot fluid was cooled down in the high-pressure buffer. It took another $20 \mathrm{~s}$ to reduce the pressure inside the cell to below the level of the low-pressure buffer. Then the low-pressure check valve opened and allowed the fluid to flow into the cell resulting in a reduction of the pressure in the low-pressure buffer. The low pressure went up at the end of the cycle as the in-flow of the cell becomes smaller than the flow through M2.

The performance of the baseline cell (Cell 3) and that of the radial conductance enhanced cells (Cells 4 and 5) are compared in Figure 6.14. The Cells 4 and 5 generated higher mass flows than Cell 3 by a factor of $8.7 \%$ and $7.2 \%$, respectively. By examining the pressures the experiment shown in Figure 6.15, in Cell 4, the high pressure started to rise earlier and had a higher peak than that in Cell 3. So, the pressure was built up faster in the compression phase, and a higher out-flow rate was obtained. The out-flow phase period seemed to be shortened in Cell 4 as well as in the subsequent decompression phase (low pressure dropped earlier) thanks to the radial enhanced conductance causing a faster thermal cycling. Due to the higher radial conductance the thermal conduction loss to the heat-sink during the heating period also increases. However, the heating time of the nominal setting was short $(8.73 \mathrm{~s})$ in order to minimize these conduction losses. The negative effect of these increased conduction losses is relatively small compared to the benefit of faster thermal cycling. 


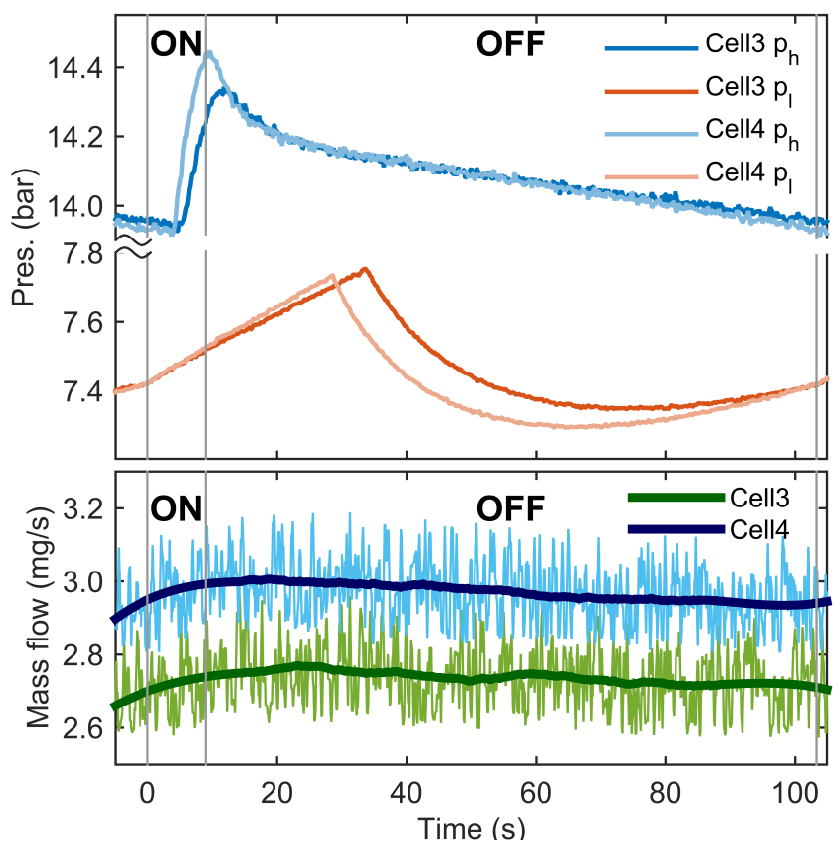

(a)

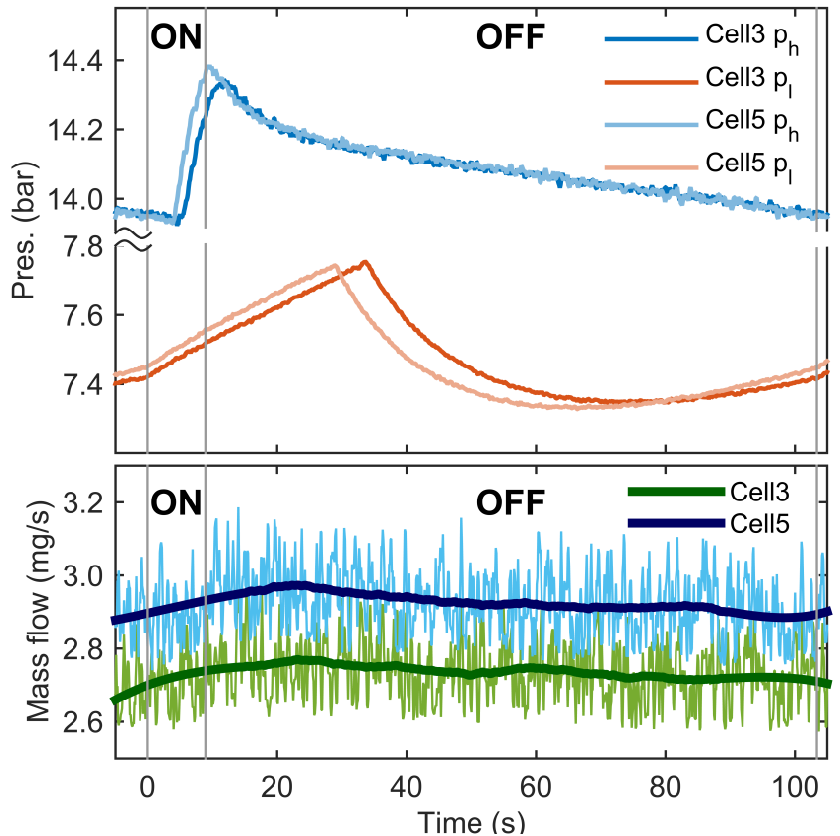

(b)

Figure 6.15. Measured pressures and mass flow rates comparison between (a) Cells 3 and 4; (b) Cells 3 and 5 in a particular cycle. 


\subsubsection{Comparison of single-cell experiments with simulations}

Operating parameters such as pressures, average heat-sink temperature, heating power, timing and compressor-cell dimensions were input into the 1-dimensional dynamic model presented in Chapter 5 for simulating the performance of the baseline cell. The simulation showed that the baseline cell can generate $2.56 \mathrm{mg} / \mathrm{s}$ at nominal conditions, which is $6.2 \%$ less than the measured value. Further relating experimental results to simulations, the periods of the compression-cycle phases are compared in Table 6.2. The measured compression (5.50 s v.s. $5.34 \mathrm{~s})$ and decompression periods ( $21.50 \mathrm{~s}$ v.s. $21.40 \mathrm{~s})$ are close to the simulated ones. However, the out flow stopped $2.7 \mathrm{~s}$ earlier and the actual out-flow time is $30.4 \%$ less compared to the simulations. This will mostly be due to the fact that in the simulations the high pressure is considered to be constant, whereas in reality in the compression phase, due to the heat pulse, the high pressure rapidly increases and peaks. In that phase, the experimental peak out-flow is 53\% higher than in the simulation. Since the in-flow phase timing is more or less determined by the cycle time, there is a relatively small difference between the experiment and the simulation.

The fact that the experimental mass-flow rate is $6.2 \%$ higher than the simulated one can be caused by a number of reasons:

Underestimation of the adsorption capacity of the carbon: The isotherm measurement normally has an uncertainty of $5-15 \%$. Because of that, it is possible that the saran carbon that we used has a larger difference in adsorption between the low-temperature, low-pressure point (point a in Figure 2.6) and the high-temperature, high-pressure point (point $\mathrm{c}$ in Figure 2.6) than assumed in the simulations. The simulated performance of the sorption cell is directly related to the isotherm data. To investigate the influence of this inaccuracy, a scaling factor was applied in the calculation of the adsorbed amount to simulate a deviation of the actual adsorption with respect to the calculated one. Figure 6.16 presents the mass flow rate per cell as a function of this scaling factor. The performance (mass flow rate per cell) increases linearly as the scaling factor increases up to 1.25. The sensitivity is about $0.0168(\mathrm{mg} / \mathrm{s}) / \%$ (or $0.66 \% / \%$ ). This means that a $9.4 \%$ higher adsorption capacity would be needed to explain a $6.2 \%$ higher flow rate.

Overestimation of the heat capacity of the adsorption mixture: In the helium sorption compressor operation, the heat capacity of the saran carbon $(<70 \%)$ and that of the

Table 6.2. Comparison between the measured and simulated periods of four phases in a sorption compression cycle (Cell 3).

\begin{tabular}{lcc}
\hline Time $(\mathrm{s})$ & Measured $($ Cell 3) & Simulated \\
\hline Compression & $5.50(+3.00 \%)$ & 5.34 \\
Out-flow & $6.50(-30.41 \%)$ & 9.34 \\
Decompression & $21.5(+0.47 \%)$ & 21.40 \\
In-flow & $70.10(+3.82 \%)$ & 67.52 \\
\hline
\end{tabular}



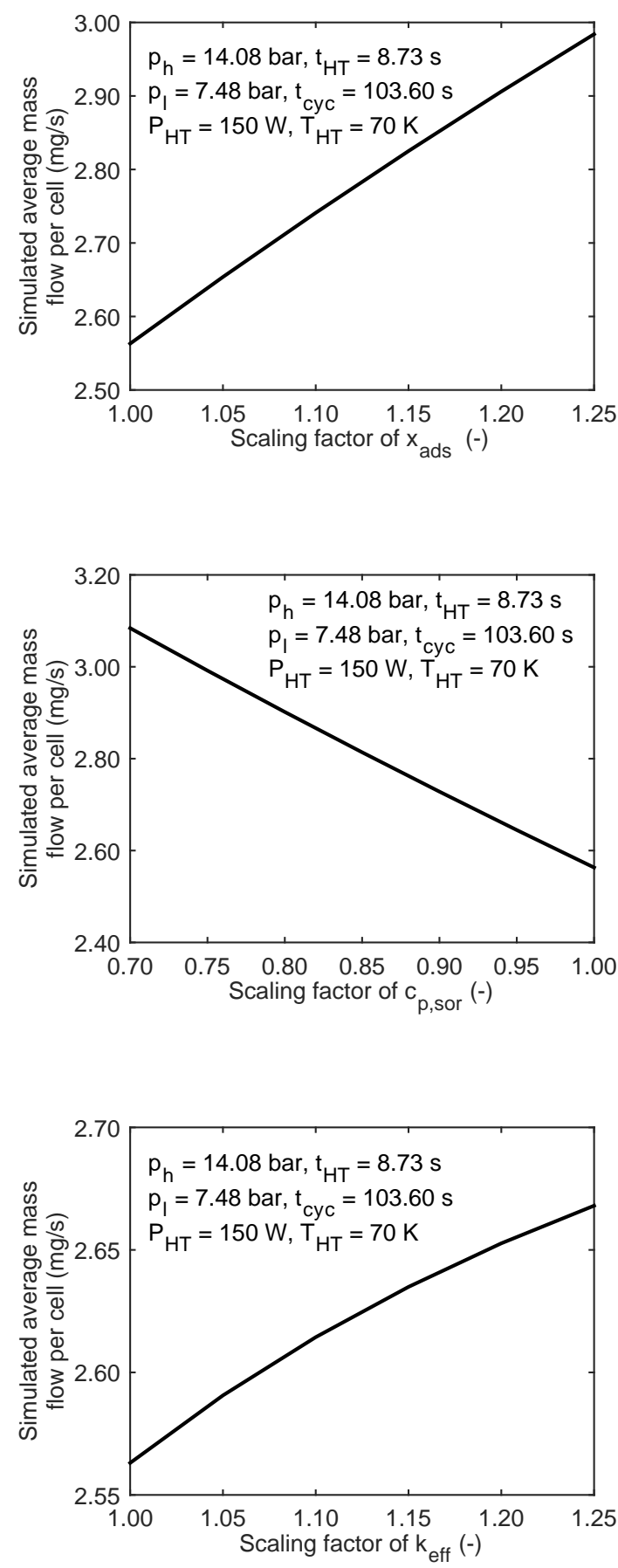

Figure 6.16. Simulated average mass flow rate per cell as a function of the adsorption amount scaling factor.

Figure 6.17. Simulated mass flow rate per cell as a function of the heat-capacity scaling factor.

Figure 6.18. Simulated average mass flow rate per cell as a function of an effective thermal conductivity scaling factor of the adsorbent mixture. 
helium in the adsorbed phase (20-30\%) dominate the total heat capacity of the adsorption mixture. If these are overestimated in the simulation, the adsorption mixture would be cycled in a lower temperature difference resulting a lower efficiency. In order to evaluate this effect, we simulated the performance with reduced heat capacity of the carbon and the adsorbed helium again by introducing a scaling factor. As shown in Figure 6.17, the sensitivity is about $0.0174(\mathrm{mg} / \mathrm{s}) / \%$ (or $0.68 \% / \%$ ). Thus, a $9.1 \%$ lower heat capacity corresponds to a $6.2 \%$ higher flow rate.

Underestimation of the effective thermal conductivity of the adsorption mixture: A higher thermal conductance in the adsorption mixture would result in a more evenly distributed temperature and would make the utilization of the adsorbent material more efficient. Consequently, it would improve the performance of the sorption compressor cell. It was proved not only theoretically but also experimentally by inserting aluminum foil between the carbon pills as discussed in Sec. 6.4.3. Therefore, if the effective thermal conductivity of the adsorption mixture was underestimated in the model, the simulation would naturally give a lower performance than expected. Figure 6.18 presents the simulated average mass flow rate per cell as a function of an effective thermal conductivity scaling factor that was applied to the adsorption mixture. Note that this effect is not as strong as the previous two effects (at maximum $0.21 \% / \%$, or $0.0055(\mathrm{mg} / \mathrm{s}) / \%$ ). Thus, the thermal conductivity in reality would need to be $30 \%$ higher to fully account for the $6.2 \%$ higher flow rate.

Neglecting the fluid dynamics in the cell during the out-flow phase: A significant difference between the experiment and simulation occurs in the out-flow phase as the peak out-flow may cause complicated fluid dynamics in the cell that may be not negligible. The convection effect of the fluid in the out-flow phase may be beneficial in distributing the temperature more evenly and thus improving the performance. This effect cannot be evaluated by simulations as it requires a multi-dimensional model that includes the fluid dynamics. This is not available and outside the scope of this thesis but this effect can be investigated further in future.

\subsubsection{Performance sensitivity}

Increasing the operating frequency, or rotational speed of a rotary compressor compared to its optimum setting, will increase its capacity but at the same time degrade its efficiency. The same applies to a sorption compressor although it operates at a very low frequency compared to the $50-60 \mathrm{~Hz}$ that is usually found in mechanical compressors. Changing the sorption compressor frequency was tested by varying the cycling time. Here, the compressor efficiency is defined by the ratio of the mass flow rate and the average input power since the pressures and the heat-sink temperature are all the same in all cases. Table 6.3 lists the performance of Cell 3 at different cycle time settings. Cases 29 and 30 are operating at - and $+10 \%$ cycle time, respectively compared to Case 27 which shows the performance at nominal conditions. In order to have the same heating energy, the 
Table 6.3. Sorption compressor performance within different cycle time (Cell 3).

\begin{tabular}{llcccccc}
\hline Case & Description & $P_{H T}, \mathrm{~W}$ & $t_{H}, \mathrm{~s}$ & $t_{c y c}, \mathrm{~s}$ & $P_{a v g}, \mathrm{~W}$ & $\dot{m}, \mathrm{mg} / \mathrm{s}$ & $\mathrm{Eff.}, \mathrm{mg} / \mathrm{J}$ \\
\hline 27 & Nominal conditions & 150 & 8.73 & 103.60 & 12.64 & 2.73 & 0.216 \\
29 & $-10 \% t_{c y c}$ & 150 & 8.73 & 93.24 & $14.04^{+11.1 \%}$ & $2.89^{+5.9 \%}$ & $0.206^{-4.6 \%}$ \\
30 & $+10 \% t_{c y c}$ & 150 & 8.73 & 113.96 & $11.49^{-9.9 \%}$ & $2.55^{-6.6 \%}$ & $0.221^{+2.3 \%}$ \\
28 & $-10 \% P_{H T} \&+11.11 \% t_{H}$ & 135 & 9.70 & 103.60 & $12.64^{+0.0 \%}$ & $2.70^{-1.1 \%}$ & $0.214^{-0.9 \%}$ \\
\hline
\end{tabular}

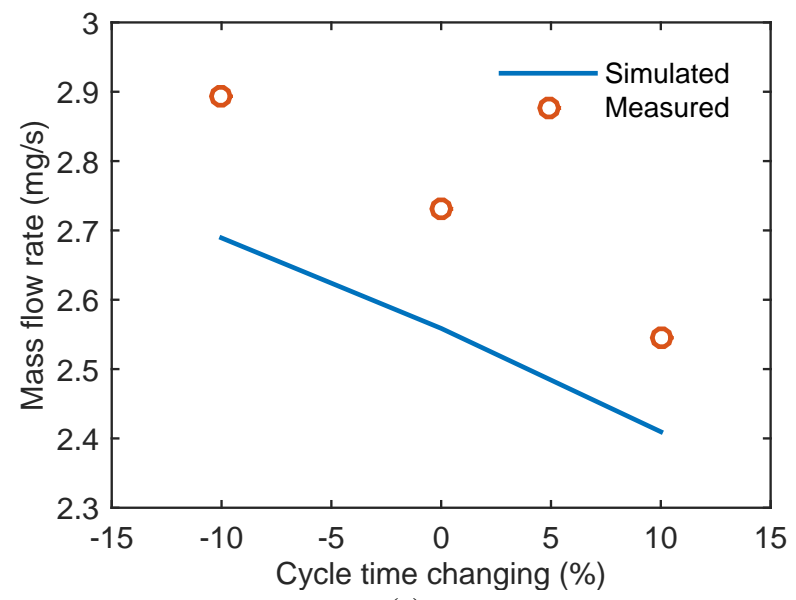

(a)

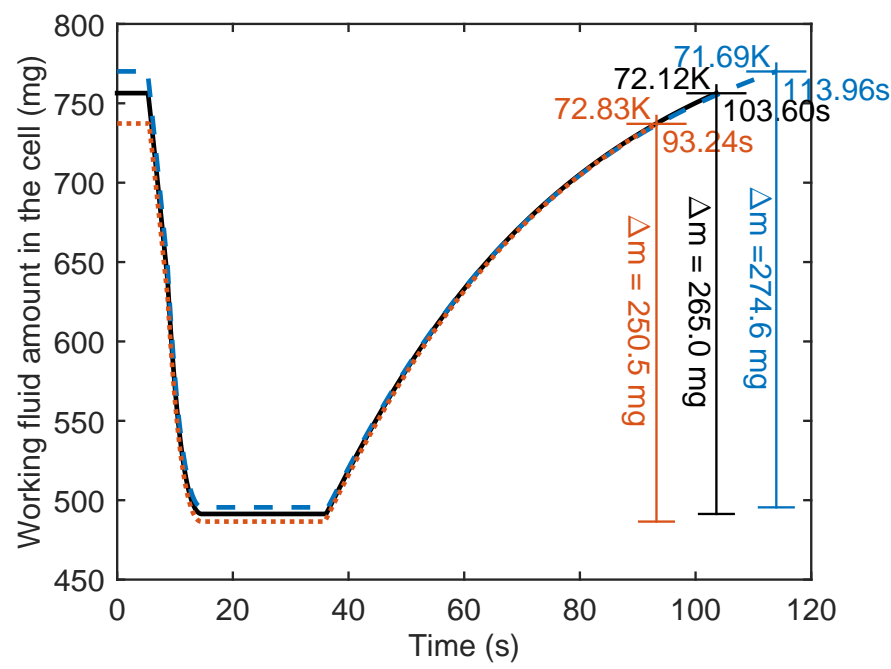

(b)

Figure 6.19. Changing cycle time experiment comparing to the simulation result. (a) Measured mass flow rate as a function of the cycle time changing based on the cycle time setting in the nominal condition; (b) Simulated fluid amount in the cell during the cycle for different cycle time. 
average input powers were $+11.1 \%$ and $-9.9 \%$ for Cases 29 and 30, respectively. At faster cycling, the compressor capacity, i.e. the mass-flow rate, improved but the corresponding efficiency degraded. Cycling slower yields a higher efficiency $(+2.3 \%$ in Case 30$)$ but generates less mass flow which means that more cells are required for a specific cooling capacity. The lower efficiency at faster cycling is because there is not sufficient time for full adsorption during the in-flow phase.

Figure 6.19 (a) presents the comparison between the experimental data and the simulation results when changing the cycle time. Figure 6.19 (b) shows the simulated amount of working fluid in the cell as a function of time. The minimum amounts when the cell is at its highest temperature are not too much different. However, at $10 \mathrm{~s}$ more cooling down time (slow cycling), the cell can be further cooled down from $72.12 \mathrm{~K}$ to $71.69 \mathrm{~K}$ thus adsorbing $10 \mathrm{mg}$ more of the working fluid. Similarly, the adsorption is $15 \mathrm{mg}$ less at a $10 \%$ shorter cycle time.

As mentioned in Chapter 3, the heat-sink temperature of the sorption compressor determines the low-temperature level of the sorption compressor cycle and thus strongly influences the compressor performance. The sensitivity of the helium compressor efficiency with respect to the heat-sink temperature is up to $5.75 \%$ per kelvin according to the simulation results as presented in Figure 6.20. In order to verify that, Cell 3 and 4 were cycled out of phase at various heat-sink temperatures from 67 to $72 \mathrm{~K}$ while the other settings were not changed. The measured sorption compressor performance, i.e. the normalized mass flow rate, in this case, is marked as diamond symbols in Figure 6.20 and has a very good match to the simulation results.

Overall, the 1-D dynamic model appears to simulate the compressor operation very well. Deviations between simulation and experimental results are measured of a few

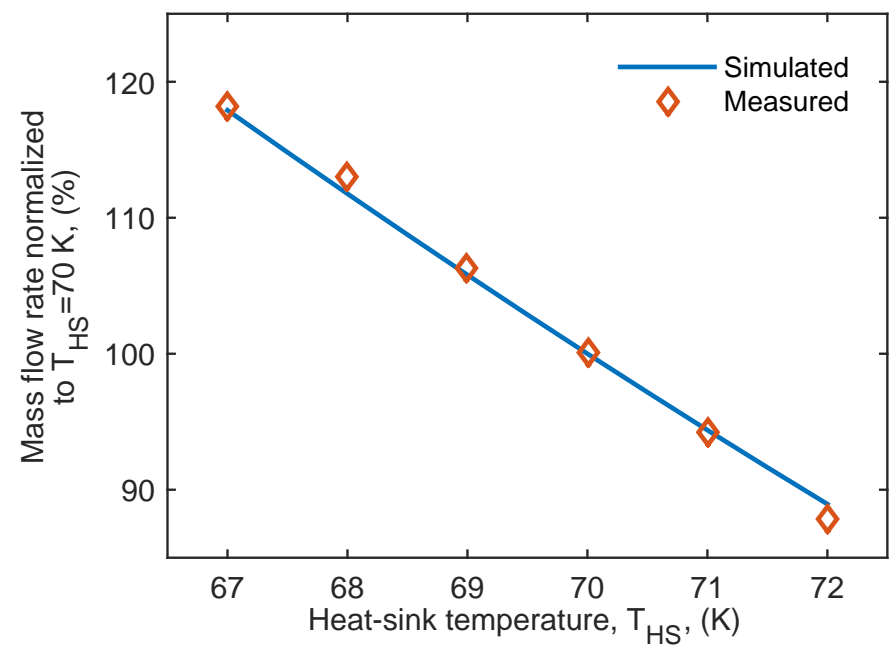

Figure 6.20. Normalized mass flow rate against the heat-sink temperature. 


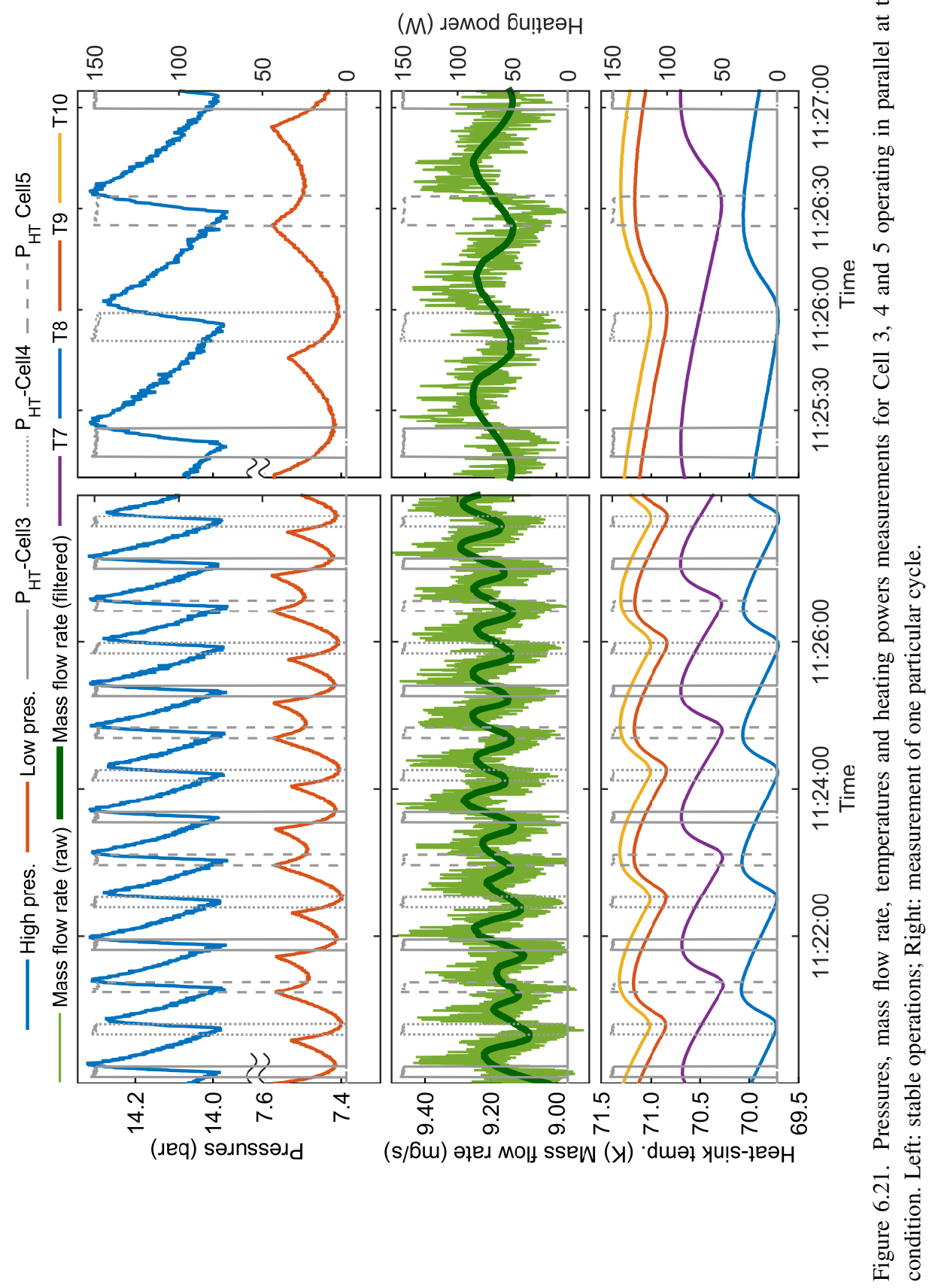


percents and can fully be accounted for by inaccuracies in model input parameters such as isotherm data, specific heat, and thermal conductivity. Despite the sensitivity to these input parameters, the model is very useful for design purposes.

\subsubsection{Multi-cells operation}

Cells 3, 4 and 5 were cycled together with $120^{\circ}$ phase difference. The stable operation status is plotted in Figure 6.21. The pressures oscillated 3 times faster than in the singlecell case. The amplitude of the high-pressure oscillation did not reduce because the outflow period is very short, i.e. $10 \%$ of the total cycle. In order to reduce the high-pressure swing more than 10 cells would be needed. In that case, the out-flow periods of the cells would overlap. The low-pressure swing, however, was reduced since the in-flow period of the cells is almost half of the cycle and thus significantly overlaps when three cells are used. The faster variation of the high pressure at equal amplitudes resulted in a larger instability of the flow rate (as a result of the operation of mass-flow controller M2 as discussed above). With an average heat-sink temperature of $70.4(70.4 \pm 0.5) \mathrm{K}$, a total average flow rate of $9.19(9.19 \pm 0.08) \mathrm{mg} / \mathrm{s}$ was achieved. In the multi-cell operation, the oscillation at the low-pressure side was reduced compared to the single-cell operation. Therefore, the total flow rate is higher than the sum of the flow rates that were measured in the single-cell operation. According to the flow rate we measured, the helium stage of the METIS cooler chain would require only 35 cells instead of 48 in the design.

\subsection{Conclusions}

A down-scaled helium sorption compressor for the METIS cooler was designed, fabricated, assembled and tested. It consists of four compressor cells connected in parallel with individual check valves. Two cells are made following the baseline cell design, one cell of which, unfortunately, could not be used because of a heater defect. The other two cells have an enhanced radial conduction by inserting aluminum foil between the carbon pills. The baseline cell can produce a mass flow rate of $2.73 \mathrm{mg} / \mathrm{s}$ between $14.08 \mathrm{bar}$ and 7.48 bar with a heat-sink temperature of $70 \mathrm{~K}$. The improvement by increasing the effective conductivity of the adsorbent material was verified: the other two cells performed better in the same setting by a factor of $8.7 \%$ and $7.2 \%$.

Furthermore, the sorption compressor performance against the cycling time and the heat-sink temperature was investigated. As the cycle time is increased, the cell can cool down closer to the heat-sink temperature and thus more gas is adsorbed. As a result, the efficiency increases but because of the longer cycle time, the average mass-flow rate decreases. Thus, a trade-off can be made between efficiency and number of cells needed for a specific cooling power. The heat-sink temperature has a strong effect. The efficiency changes close to $6 \%$ per kelvin of change in heat-sink temperature. The 1-D dynamic 
model described in Chapter 5 appears to simulate the compressor operation very well. Deviations between simulation and experimental results are measured of a few percent and can fully be accounted for by inaccuracies in model input parameters such as isotherm data, specific heat, and thermal conductivity. Despite the sensitivity to these input parameters, the model is very useful for design purposes. Finally, the compressor was operated with three cells running $120^{\circ}$ out of phase, and a total mass flow rate of $9.2 \mathrm{mg} / \mathrm{s}$ was achieved. Thus, the required flow rate of $105.8 \mathrm{mg} / \mathrm{s}$ in the METIS cooler would require 35 cells instead of the 42 cells in the design. 



\section{Scaled $40 \mathrm{~K}$ Neon-based Sorption Joule-Thomson Cooler}

In this chapter, we presents the design of the scaled $40 \mathrm{~K}$ neon sorption JT cooler that is to demonstrate the feasibility of the sorption cooler technology for the METIS instrument. The preliminary breadboard testing performed on the JT cold stage is discussed, and the current status of this cooler is stated. 


\subsection{Overview of the scaled $40 \mathrm{~K}$ neon-based sorption JT cooler}

The scaled $40 \mathrm{~K}$ neon sorption cooler was designed and built to demonstrate the feasibility of the sorption cooler technology for the METIS instrument. It is designed to produce $1.0 \mathrm{~W}$ cooling power at $40 \mathrm{~K}$ with a heat-sink temperature of $70 \mathrm{~K}$. Table 7.1 lists the design specifications for this demo cooler. A $0.5 \mathrm{~K}$ temperature difference is assumed for the $40 \mathrm{~K} \mathrm{CHX}$ to transfer the cooling power, therefore the low pressure is reduced to 13.6 bar corresponding to the boiling temperature of $39.5 \mathrm{~K}$. A design margin of $25 \%$ for the cooling power is considered resulting in a required mass flow rate of $44.5 \mathrm{mg} / \mathrm{s}$.

A CAD overview of this demo cooler is presented in Figure 7.1. It consists of two submodules that are the neon JT cold stage and the neon sorption compressor. Five sorption compressor cells are mounted on an isothermal cold platform that will be cooled down to $70 \mathrm{~K}$ by a pumped liquid nitrogen bath. Buffers and check valves are arranged in the

Table 7.1. Design specifications of the scaled neon-based sorption cooler.

\begin{tabular}{ccc}
\hline Parameters & Unit & Specification \\
\hline Cooling temperature & $\mathrm{K}$ & $40(39.5)$ \\
Cooling power & $\mathrm{W}$ & $1.0(1.25)$ \\
High pressure & $\mathrm{bar}$ & 112 \\
Low pressure & $\mathrm{bar}$ & $14.6(13.6)$ \\
Mass flow rate & $\mathrm{mg} / \mathrm{s}$ & $36.0(44.5)$ \\
Heat-sink temperature & $\mathrm{K}$ & 70 \\
\hline
\end{tabular}

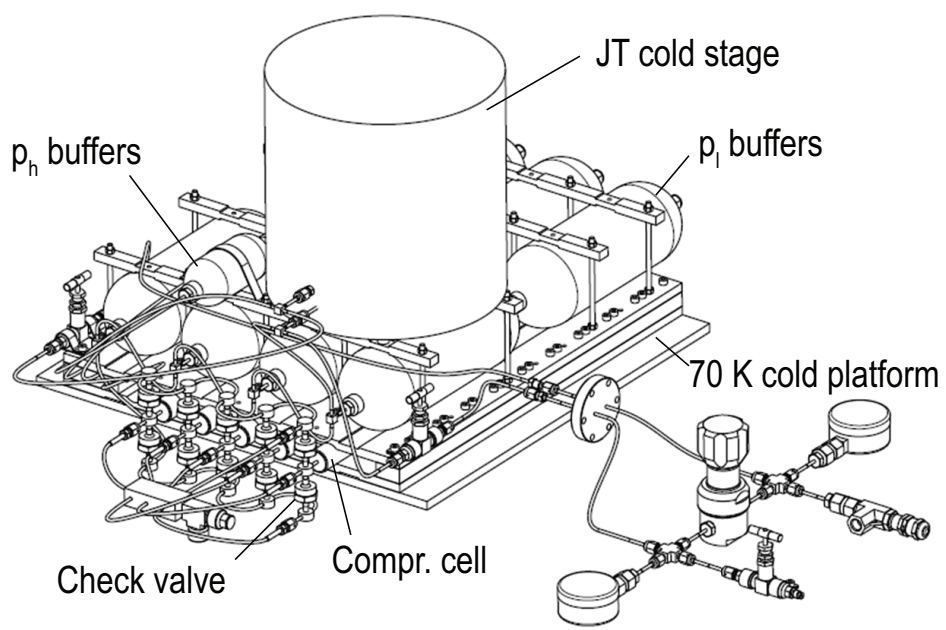

Figure 7.1. CAD overview of the scaled $40 \mathrm{~K}$ neon-based sorption cooler. 
same way as in the scaled helium sorption compressor. The JT cold stage assembly is contained in a radiation shield that is also thermally linked to the $70 \mathrm{~K}$ platform.

\section{2 $40 \mathrm{~K}$ neon cold stage design and primarily testing}

The $40 \mathrm{~K}$ neon cold stage has three components: a CFHX operating from $70 \mathrm{~K}$ to $40 \mathrm{~K}$, a JT restriction through which the high-pressure flow of 112 bar expands isenthalpicly to 13.6 bar and a $40 \mathrm{~K} \mathrm{CHX}$ that is able to extract the cooling power from the two phase neon flow with minimum temperature difference.

\subsubsection{CFHX}

Instead of using a tube-in-tube CFHX, a parallel-tube CFHX was chosen in this case. It is made of two $3 \mathrm{~m}$ long stainless steel tubes that are brazed side by side. It is designed to have an effectiveness of $98.7 \%$ with pressure drops of $0.20 \mathrm{mbar}$ for the high pressure and 0.95 mbar for the low pressure line. The thermal conduction loss through the CFHX is less than $1.0 \mathrm{~mW}$ which is negligible compared to the cooling power.

\subsubsection{Joule-Thomson restriction}

The pressure difference between the high- and low-pressure sides is almost 100 bar. Therefore, a capillary JT restriction is not suitable simply because it requires an enormous length to create such a large pressure drop with the flow rate corresponding to the design specifications.

A supersonic, orifice restriction was used in the neon JT cold stage, see Figure 7.2. It is a laser-machined hole in a stainless steel disk. The size was based on a model presented by Maytal [87]. According to this model, at an CFHX high-pressure inlet of $70 \mathrm{~K}$ at 112 bar

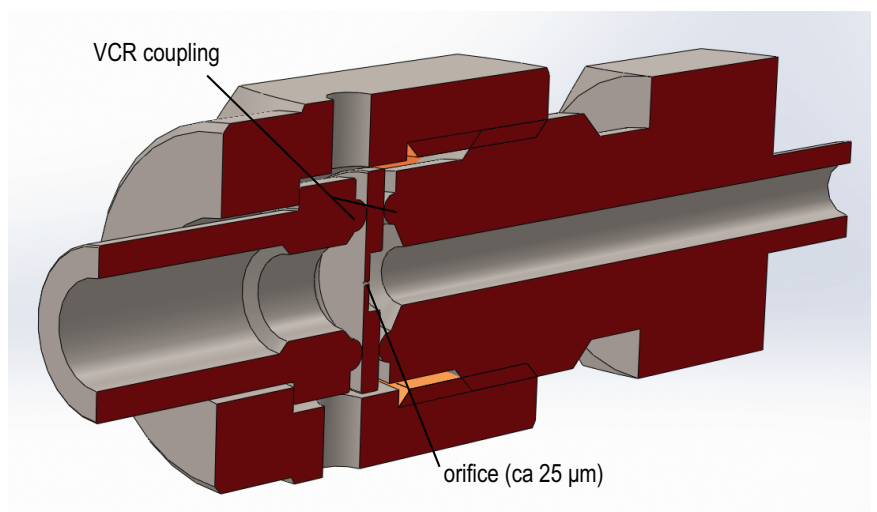

Figure 7.2. Cross-sectional view of the supersonic orifice restriction. 
and a cold-tip temperature of $40 \mathrm{~K}$, a flow of $44 \mathrm{mg} / \mathrm{s}$ can be realized with a hole diameter of $23 \mu \mathrm{m}$. After production the hole diameter was optically measured to be $23.3 \mu \mathrm{m}$. Flow experiments were performed with this restriction at $300 \mathrm{~K}$ and at $77 \mathrm{~K}$ with helium, nitrogen and neon. In these experiments the gas was vented to atmosphere and the high pressure was varied between roughly 30 bar and 90 bar. The measured flow in all cases was compared with the modeled flow and the best fit was obtained for a $25 \mathrm{~m}$ diameter hole in the model. Using this $25 \mu \mathrm{m}$, at the operating conditions of the neon cold stage, i.e. CFHX high-pressure inlet of $70 \mathrm{~K}$ at 112 bar and cold-tip at $40 \mathrm{~K}$, the model predicts a flow rate of $52 \mathrm{mg} / \mathrm{s}$ (scaling with the area of the restriction).

\subsection{3 $40 \mathrm{~K} \mathrm{CHX}$}

A moderate heat transfer coefficient of $0.1 \mathrm{~W} /\left(\mathrm{cm}^{2}-\mathrm{K}\right)$ was chosen for the $40 \mathrm{~K} \mathrm{CHX}$ design. It is based on free convection in the neon [88]. In reality, however, this coefficient will be higher due to the forced convection. With $0.5 \mathrm{~K}$ temperature difference for heat transfer, the $\mathrm{CHX}$ requires $20 \mathrm{~cm}^{2}$ heat transfer area for $1.0 \mathrm{~W}$.

The details of the $40 \mathrm{~K} \mathrm{CHX}$ module is shown in Figure 7.3. The CHX module is made of stainless steel tubing with a copper insert. The copper insert is a cylinder with micro grooves machined by EDM. These grooves are $400 \mu \mathrm{m}$ wide and create micro flow channels with a hydraulic diameter of $800 \mu \mathrm{m}$. The heat transfer area in such a module is $10.8 \mathrm{~cm}^{2}$. The stainless steel tube is clamped in an aluminum base block that is mount on the $40 \mathrm{~K}$ cold plate. Two such CHX modules are used to provide $1.0 \mathrm{~W}$ heat transfer.

\subsubsection{Cold stage assembly}

Figure 7.4 shows the $40 \mathrm{~K}$ neon cold stage assembly. The CFHX is bend into a helical coil. The entire cold stage is suspended in a radiation shield frame by 20 Kevlar wires.

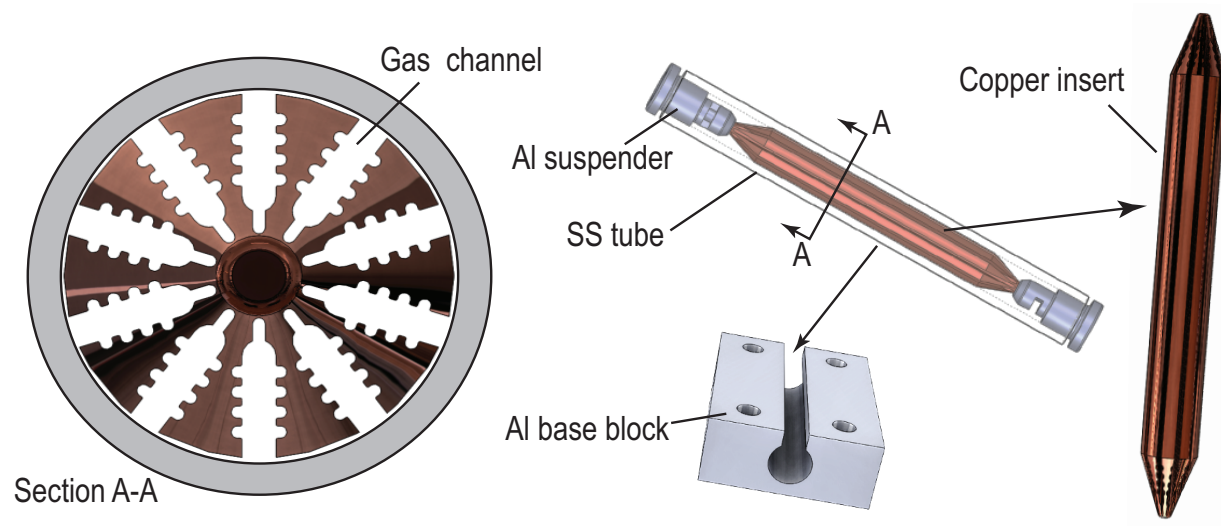

Figure 7.3. $40 \mathrm{~K}$ CHX module. 


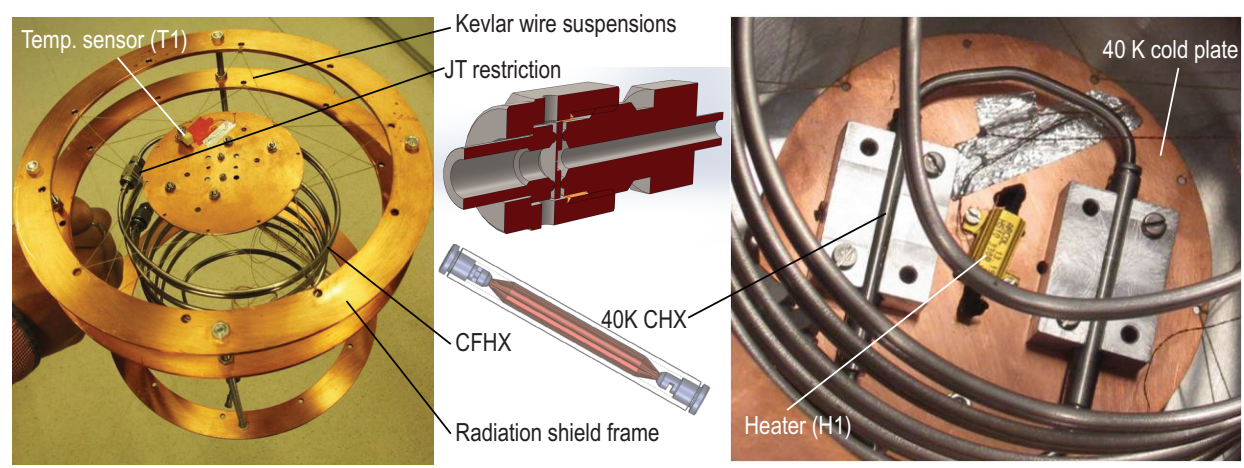

Figure 7.4. $40 \mathrm{~K}$ neon cold stage assembly.

Each suspending wire has a diameter of $0.3 \mathrm{~mm}$ thick and is $70 \mathrm{~mm}$ long. The conduction loss through the suspension is roughly $0.6 \mathrm{~mW}$. A temperature sensor and a heater are mounted on the $40 \mathrm{~K}$ cold plate for measuring the operating temperature and cooling power.

\subsubsection{Experimental verification}

The neon JT cold stage was tested in an open loop system. Figure 7.5 is a schematic of the test setup. The high-pressure neon flow is provided by a neon gas bottle with pressure

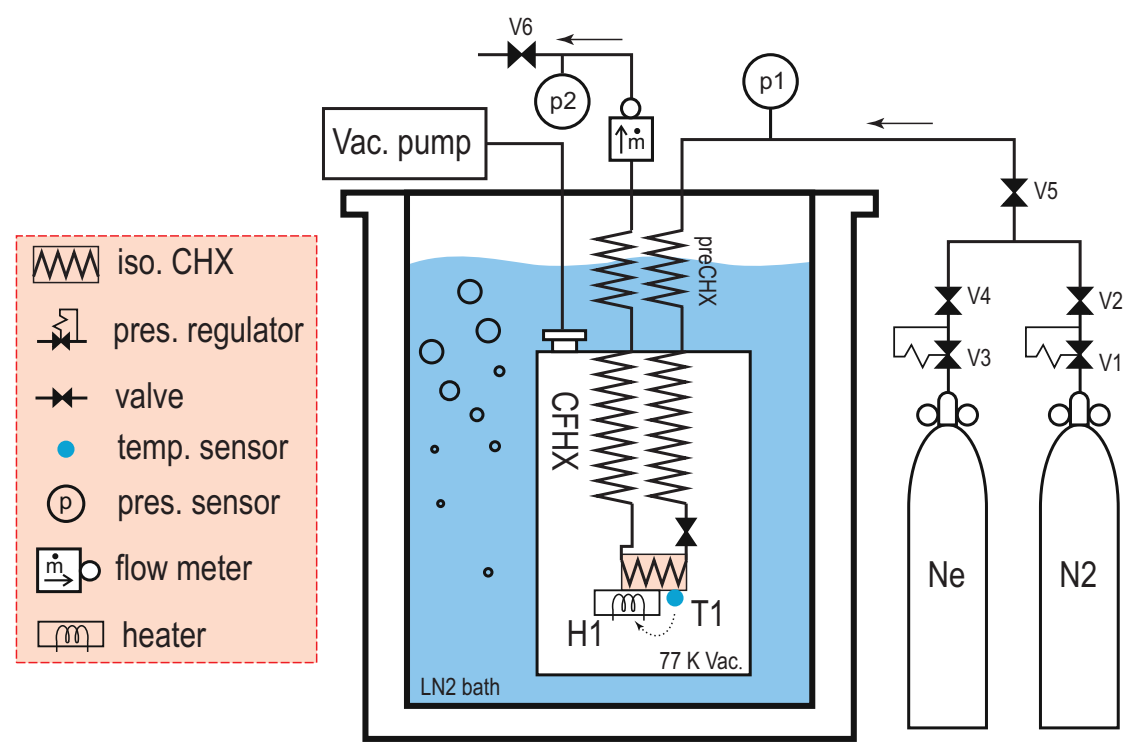

Figure 7.5. Schematic of the neon JT cold stage test setup. 
regulator (V3) and then precooled by a liquid nitrogen bath before entering the JT cold stage. The low-pressure flow is regulated by a valve (V6) before being exhausted into the atmosphere. The cold stage assembly is placed in a vacuum chamber that is submerged in the liquid nitrogen bath. Since the warm-end temperature of the CFHX in this setup is $77 \mathrm{~K}$ instead of $70 \mathrm{~K}$, the exit temperature of the high-pressure line (i.e. the JT inlet) is higher. At ideal operation of the CFHX (i.e. maximum enthalpy exchange) this would be $55 \mathrm{~K}$ instead of $52 \mathrm{~K}$. Because of the lower density at higher temperature, the mass flow through the restriction is lower. Based on Maytals model [87] and our test experiments we expect $48 \mathrm{mg} / \mathrm{s}$ instead of $52 \mathrm{mg} / \mathrm{s}$ as mentioned in Sec. 7.2.2, with a difference by about $10 \%$.

Figure 7.6 shows the record of the measurement, presenting cooling temperature T1, flow rate, cooling power, and pressures. Figure 7.7-Figure 7.10 are four zoomed sections of interest as indicated in Figure 7.6.

In order to save neon gas, the cold stage was first precooled to $77 \mathrm{~K}$ using nitrogen as the working fluid. The supply high pressure was 112 bar while the exhaust nitrogen was released into atmosphere with V6 fully open. It took about 5 hours to cool the cold stage from room temperature to $83 \mathrm{~K}$ (T1 in Figure 7.5), see Figure 7.7. Next, the working fluid was switched to neon, indicated with a in Figure 7.8. Next, the JT cold stage encountered a clogging issue and the flow rate dropped to zero while T1 started to increase ("b" in Figure 7.8). This is most probably caused by frozen impurities such as water and $\mathrm{CO}_{2}$ accumulated at the JT restriction. We applied $2.3 \mathrm{~W}$ heating power to warm up the cold plate hoping to depose the impurities ("c" in Figure 7.8). The cold stage was declogged as T1 reached $129 \mathrm{~K}$, the flow rate then suddenly rose to $18.5 \mathrm{mg} / \mathrm{s}$ ("d" in Figure 7.8). The heating was switched off and the cold stage started to cool down again. However, the cooling-down speed was merely $4.2 \mathrm{~K} / \mathrm{hr}$ since the flow rate at this high JT temperature was only $17-18 \mathrm{mg} / \mathrm{s}$ and the heat capacities of the materials are still high (>120 K). The neon bottle ran out quickly and its supply pressure started to drop ("e" in Figure 7.8). To accelerate the cooling, the vacuum chamber was filled with helium as a contact gas so that the liquid nitrogen bath was utilized to help cooling the cold stage when its temperature was still higher than $77 \mathrm{~K}$ ("f" in Figure 7.8). The cooling speed was increased significantly by a factor of 17.8 , to $75.0 \mathrm{~K} / \mathrm{hr}$. The mass flow rate increased consequently as the temperature decreased. As the temperature approached $77 \mathrm{~K}$, the cooling speed started to decrease. When $\mathrm{T} 1$ was $70 \mathrm{~K}$, the vacuum chamber was pumped again since the cold stage got colder than the liquid nitrogen bath ("g" in Figure 7.8). The cooling speed then further increased and it took 26 minutes to cool from $70 \mathrm{~K}$ to $40 \mathrm{~K}$ with the high pressure decreasing gradually from 94.0 to 89.4 bar and the flow rate increasing from 25.3 to $39.4 \mathrm{mg} / \mathrm{s}$.

A full neon bottle was connected to the system to provide sufficient high pressure ("a" in Figure 7.9). The cooling temperature $\mathrm{T} 1$ was set to $40 \mathrm{~K}$ to measure the cooling power. V6 was fully open resulting in a low pressure of $1 \mathrm{~atm}$. Next, the temperature control was switched off for about 11 minutes and the low pressure was regulated to 12.0 bar by 


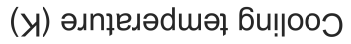

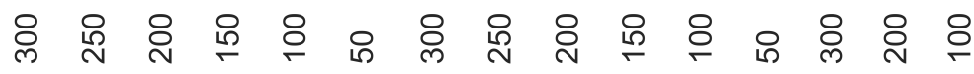

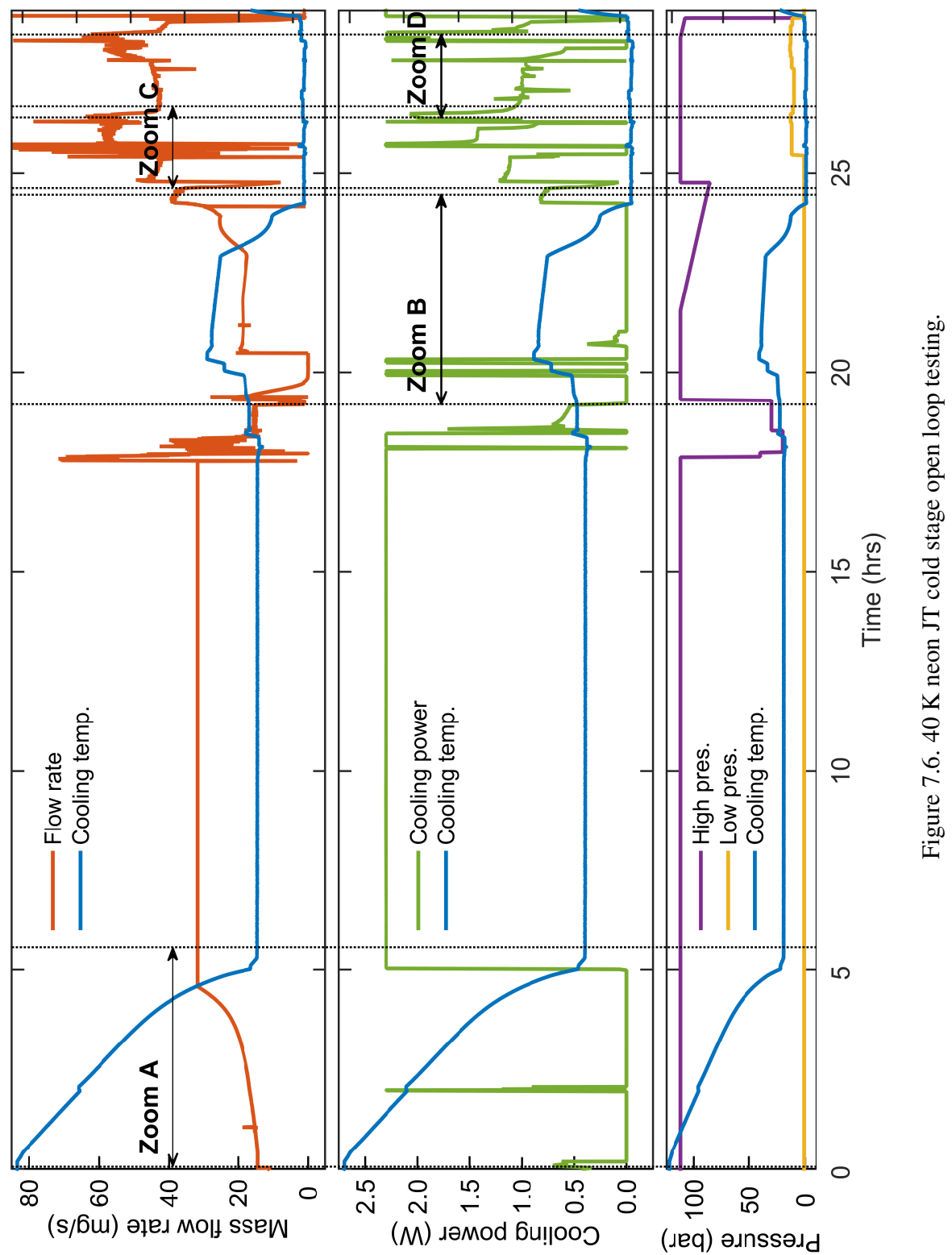




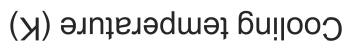

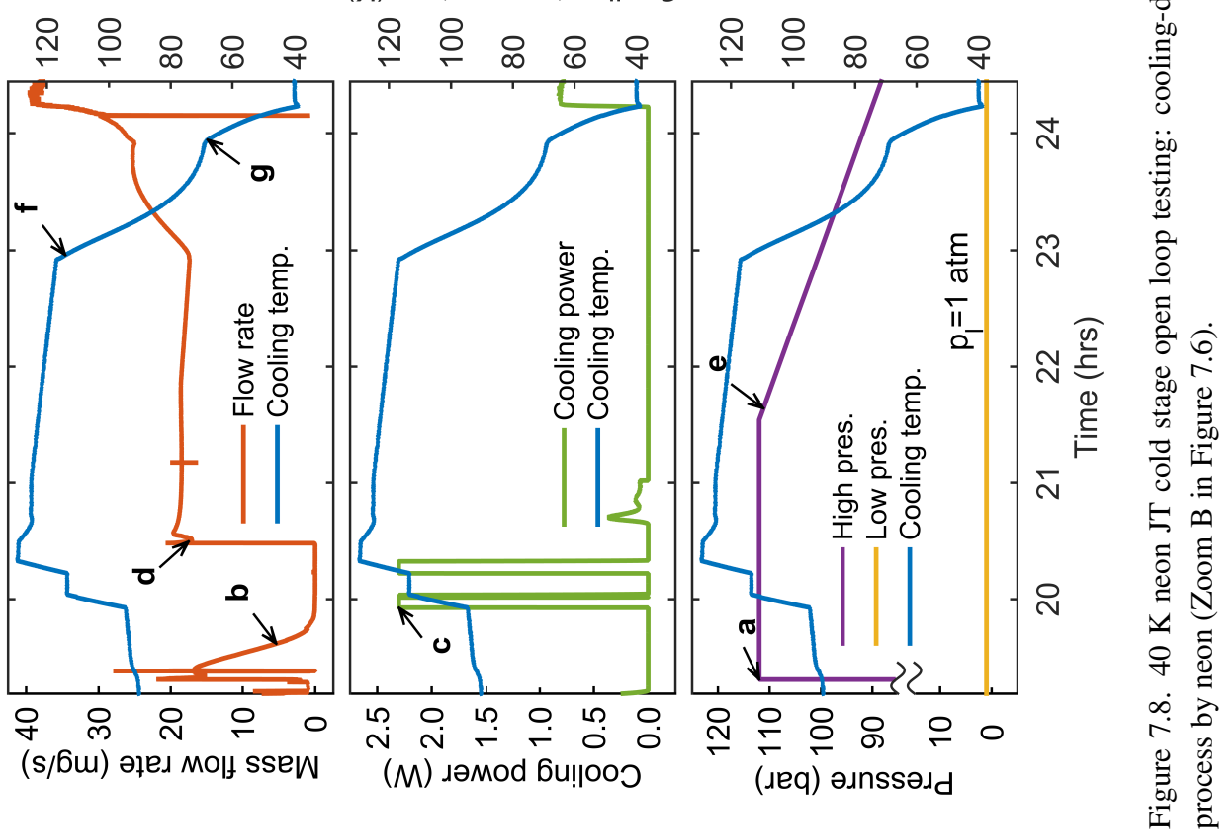

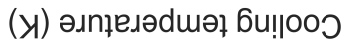

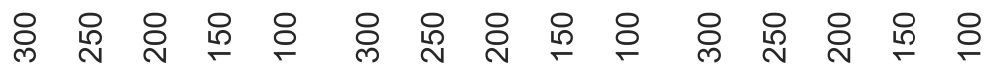
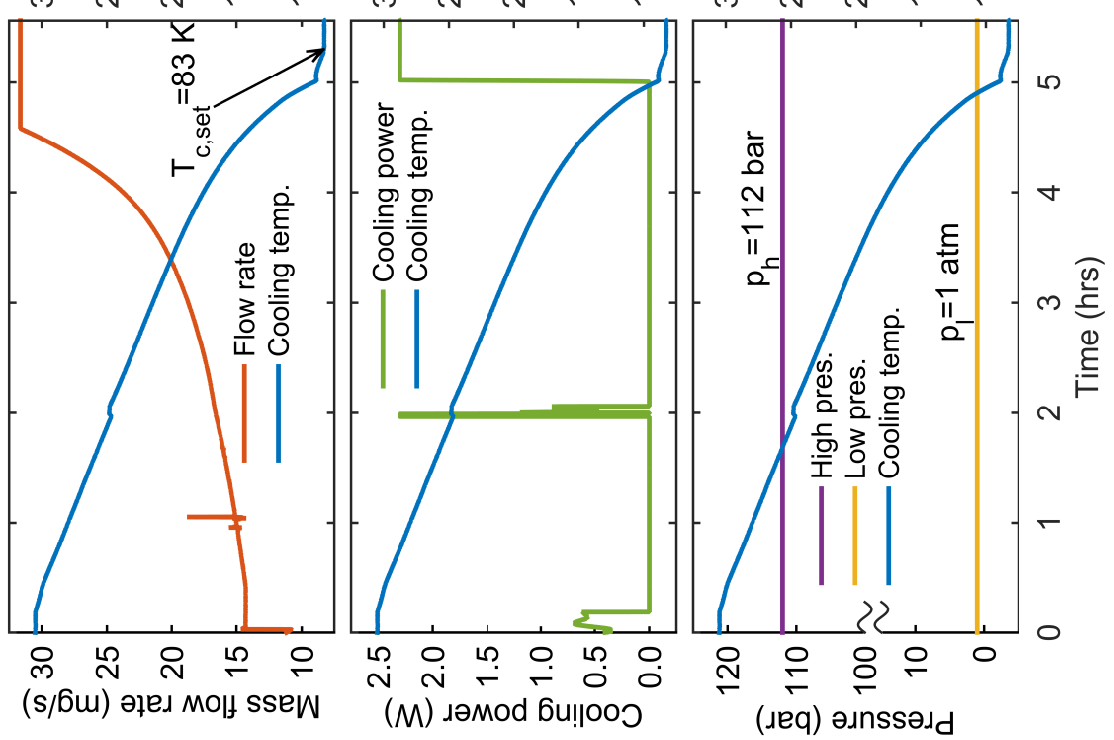

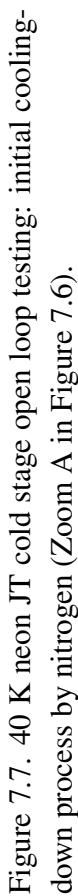




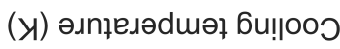

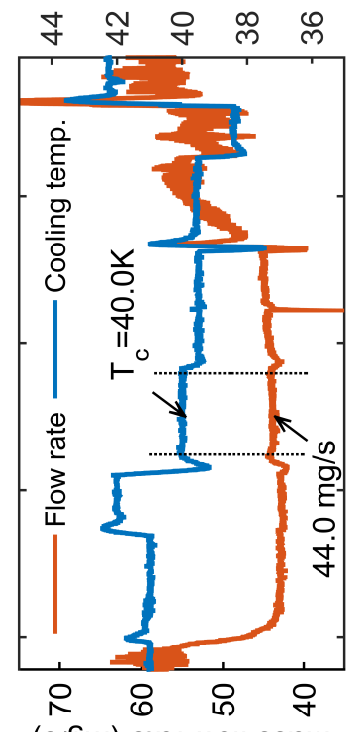

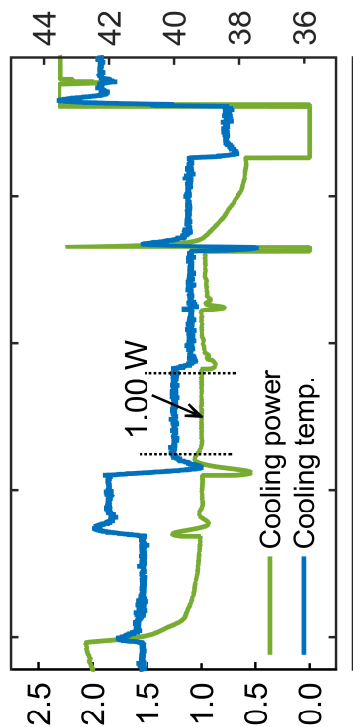

(M) ләмоd 6u!jooว

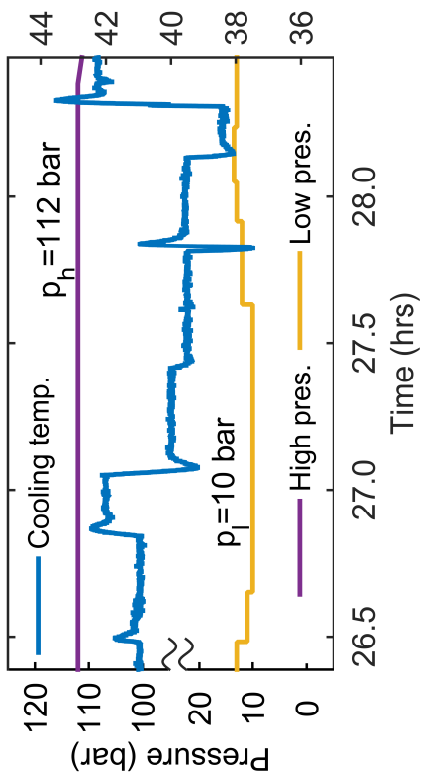

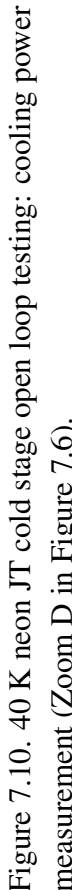

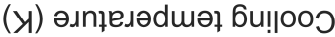

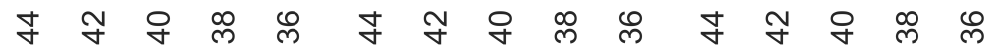
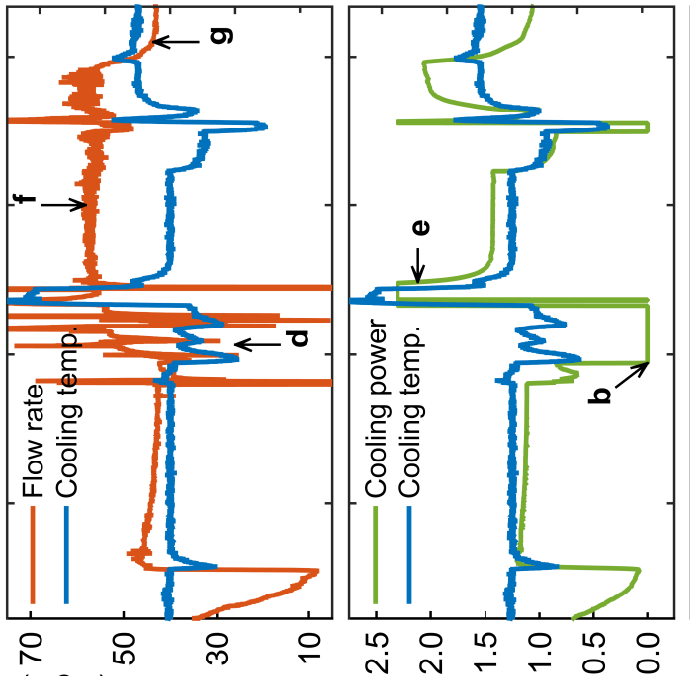

(M) ләмоd bu!ाooว

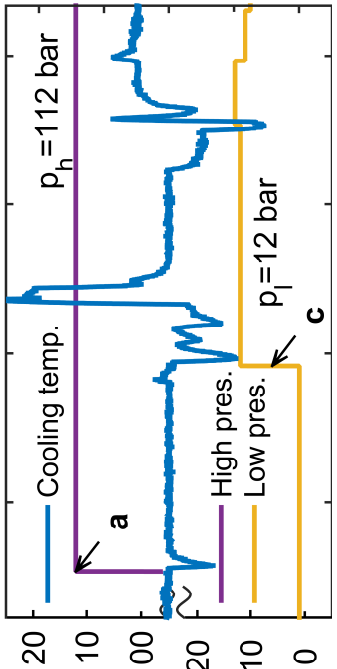

(ıeq) әınssəגd

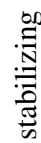

in

응

$\frac{1}{0}$

วิ

๑

욜ㄹ

5

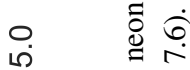

ลั่

$\forall$

우

a. $\Xi$

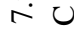

壳 
tuning V6 ("b" and "c" in Figure 7.9). Then the liquid was accumulated in the CHX and partly returned to the CFHX causing instability in the flow rate and the temperature (" $\mathrm{d}$ " in Figure 7.9). Once the temperature control was switched on again, a high heating peak was found because the $\mathrm{CHX}$ was filled completely with liquid neon that first needed to evaporate, at least partly. The heating power quickly returned to a moderate steady value of $1.43 \mathrm{~W}$ ("e" in Figure 7.9). At the same time the flow rate increased to $55-58 \mathrm{mg} / \mathrm{s}$ due to the evaporation of the neon liquid that was stored in the CHX (" $\mathrm{f}$ " in Figure 7.9). Moreover, in this part of the experiment the inlet of the JT will have been at a relatively low temperature because of the liquid present in the CFHX. This lower inlet temperature will also have contributed to a higher flow rate. Then, the set-point temperature and low pressure were adjusted serval times in order to achieve a steady operation with which we could measure the cooling power. The flow rate decreased to about $43 \mathrm{mg} / \mathrm{s}$ once the liquid amount in the $\mathrm{CXH}$ was low and the system reached equilibrium ("g" in Figure 7.9).

Finally, a cooling power of $1.00 \mathrm{~W}$ at $40 \mathrm{~K}$ was measured with a mass flow rate of $44.0 \mathrm{mg} / \mathrm{s}$ and a high pressure of $112 \mathrm{bar}$, as shown in Figure 7.10. The ideal gross cooling power was calculated to be $1.025 \mathrm{~W}$. The corresponding net cooling power was $0.985 \mathrm{~W}$ taking into account the CFHX inefficiency and other parasitic losses. As mentioned at the end of Sec. 7.2.5 we expected $48 \mathrm{mg} / \mathrm{s}$ based on modeling and test experiments. So, the flow is $10 \%$ lower which is quite well acceptable since in our operating conditions the neon at the JT is entering the two-phase regime and the model then gets less reliable. Furthermore, as also discussed in Sec. 7.2.5, in METIS cooler the precooling temperature is $70 \mathrm{~K}$ instead of $77 \mathrm{~K}$ and that will yield a higher flow rate by about $10 \%$. So, in operation we may expect a flow rate of about $48 \mathrm{mg} / \mathrm{s}$, corresponding to a gross cooling power of about $1.1 \mathrm{~W}$.

In order to achieve $40 \mathrm{~K}$ in our experiments, however, the low pressure had to be reduced to 10 bar corresponding to a boiling temperature of $37.54 \mathrm{~K}$, instead of the design value of 13.57 bar $\left(T_{\text {vap }}=39.5 \mathrm{~K}\right)$. This indicates that the temperature difference that is required for the $1 \mathrm{~W}$ heat transfer in the $40 \mathrm{~K} \mathrm{CHX}$ is about $2.5 \mathrm{~K}$ which is much higher than the design assumption of $0.5 \mathrm{~K}$. We expect this to be caused by a too small thermal contact between the copper insert and the stainless steel tube in the CHX. The copper insert is suspended in the stainless steel tube leaving a small gap in between. This gap will be a major thermal resistance in the thermal path from the two phase neon flow to the $40 \mathrm{~K}$ cold plate. In future, this $40 \mathrm{~K} \mathrm{CHX}$ will be redesigned.

\subsection{Neon sorption compressor design}

In Chapter 5, the main dimensions of the sorption compressor cell for the neon stage in the METIS cooler chain are listed in Table 5.2. The detailed design of the sorption compressor was carried out at the University of Twente (UT), whereas the compressor was manufactured and fabricated by Airbus Defence and Space Netherlands (ADSN). ADSN 
made some minor adaptation based on UTs original design according to the internal regulations and protocols with respect to production and safety. The diameter of the carbon pills was redesigned to be $14.29 \mathrm{~mm}$. Each pill has a height of $5.02 \mathrm{~mm}$ and 100 pills were inserted into each cell. A PTFE tube with a wall thickness of $2.82 \mathrm{~mm}$ was chosen as the insulation layer in the cell. The insulation at the ends of the cell was also made of PTFE. PTFE has a smaller thermal conductivity, similar specific heat and a larger density compared to Kapton that is used for the insulation in the UT design. This will influence the compressor performance slightly. A simulation was run to evaluate the performance of this ADSN design. The input heating power of $150 \mathrm{~W}$ per cell was fixed. The heating time is $77.97 \mathrm{~s}$, whereas the cycle time is $758.67 \mathrm{~s}$. Thus the average heat power yields $15.42 \mathrm{~W}$ for each cell. With a heat-sink temperature of $70 \mathrm{~K}$, each cell can deliver $11.16 \mathrm{mg} / \mathrm{s}$. Therefore, 4 compressor cells are needed for producing the required mass flow of $44.5 \mathrm{mg} / \mathrm{s}$. One additional cell is included for redundancy. The buffer sizes are $0.15 \mathrm{~L}$ and $8 \mathrm{~L}$ for the high pressure and low pressure, respectively. The mechanically adapted check valves (Sec. 6.2.3) were used in the neon sorption compressor.

At the time of this writing, the neon compressor is under construction, as shown in Figure 7.11. Once it is completed, the neon cold stage will be coupled to the compressor. Full-system test experiments for the entire cooler will then be carried out.

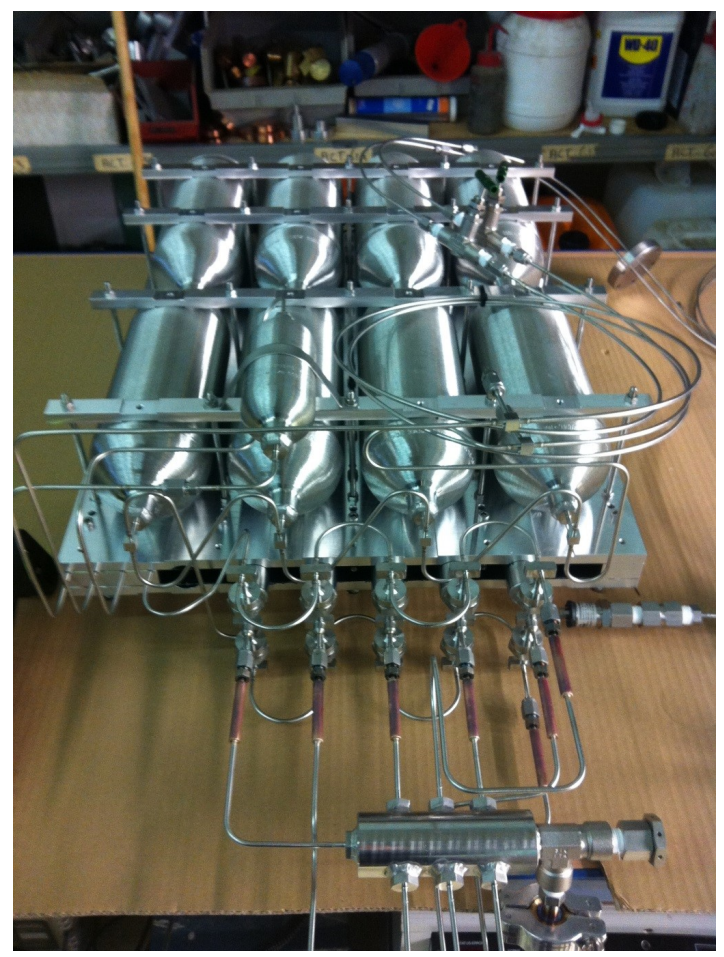

Figure 7.11. The neon sorption compressor assembly under construction at Airbus Defence and Space Netherlands. 


\subsection{Conclusions}

A $40 \mathrm{~K}$ neon sorption JT cooler setup is designed and fabricated. The cold stage of this cooler was tested in an open loop, and $1.00 \mathrm{~W}$ at $40 \mathrm{~K}$ was achieved as expected. The orifice JT restriction was verified with an inaccuracy of $8 \%$ and the CFHX also performed according to the design. However, in future the $40 \mathrm{~K} \mathrm{CHX}$ has to be redesigned to increase the thermal contact between the evaporating neon and the cold plate and thus to reduce the temperature difference required for adequate heat transfer. The compressor cells for this neon cooler demo are under construction at Airbus Defence and Space Netherlands and, after completion, these will be combined with the cold stage for full-system test experiments. 


\section{Conclusions and Outlook}

This thesis focused on the development of a vibrationless sorption cooler for the METIS instrument in the E-ELT. Starting with the knowledge and experience accumulated in earlier space-mission projects, the sorption cooling technology for this ground application was investigated theoretically. Modifications for this particular case were made based on the theoretical analysis. Experimentally, three demo setups were designed, fabricated and tested for validating the theoretical modelling. This research is summarized in this chapter. A few promising topics for future research are discussed here as well. 


\subsection{Conclusions}

The scope of the thesis is to provide vibration-free cooling at multiple cryogenic temperatures for the optics and infrared detectors in large ground telescopes. Based on the sorption cooling technology that was developed for space applications in the earlier work$\mathrm{s}$, in this thesis, we propose a sorption JT cooler chain for the METIS instrument in the E-ELT. This application requires cryogenic cooling stages at $40 \mathrm{~K}, 25 \mathrm{~K}$ and $8 \mathrm{~K}$ with respective cooling powers of $1.4 \mathrm{~W}, 1.1 \mathrm{~W}$, and $0.4 \mathrm{~W}$.

First, the thermodynamic fundamentals of the JT cold stage and the sorption compressor were introduced. Based on this thermal analysis, a general methodology for optimizing the working fluid in a sorption-based JT cooler is presented. By using this method, the working fluid of a JT cooler driven by a single-stage sorption compressor was optimized for two ranges of cold-tip operating temperatures: $65-160 \mathrm{~K}$ and $16-38 \mathrm{~K}$. In the former range, nitrogen is best at the low-temperature end, followed by carbon monoxide, methane, and krypton. The boundary temperatures depend on the heat-sink temperature. In the lower temperature range hydrogen is preferred up to $31 \mathrm{~K}$ and above that temperature neon is better. The optimization method was also extended to two-stage compression, and specifically nitrogen and carbon monoxide are considered as examples. Analogously, this method can be applied for other working fluids to find a maximum overall COP of the sorption cooler.

A numerical model for a tube-in-tube CFHX was built for design purposes. Including the longitudinal conduction and radiation losses, the model simulates the heat transfer between the flows and the tubes and the pressure drops in the flow.

A conceptual design of the METIS cooler chain was presented and impressions of configurations, size and performance were given. Three stages are thermally linked in parallel and provide cooling at $8 \mathrm{~K}$ (helium), $25 \mathrm{~K}$ (hydrogen) and $40 \mathrm{~K}$ (neon). The heatsink temperature for the sorption compressors is at $70 \mathrm{~K}$. The helium stage determines the overall input power and size of the system. It operates with a small pressure ratio (only 1.85) but consumes more than $60 \%$ of the total input power. The hydrogen stage uses a two-stage sorption compressor to compress the hydrogen from 0.12 bar to 23.7 bar. The neon stage requires a quite high optimum high pressure of almost 96 bar. In total, the cooler chain requires $1031 \mathrm{~W}$ input at $70 \mathrm{~K}$ (taking into account $25 \%$ margins in (pre)cooling powers and $10 \%$ margin added to the resulting input power).

To demonstrate the feasibility of the sorption JT technology for such a large ground application and validate the design, three demonstration setups were planned based on the baseline design of the METIS cooler chain:

\section{Full-scale $8 \mathrm{~K}$ helium JT cold stage:}

This demo is representative of the helium cold stage in the METIS cooler chain in terms of cooling power, cooling temperature and mass flow, but it is driven by a mechanical compressor. It should, therefore, be able to cool to $8 \mathrm{~K}$ with a helium mass flow rate of 
$100 \mathrm{mg} / \mathrm{s}$ precooled to a temperature of $15 \mathrm{~K}$. Precooling is supplied by a conventional mechanical refrigerator. The aim was to validate the cold stage performance, in particular regarding the $15-8 \mathrm{~K}$ counter flow heat exchanger (CFHX), the $8 \mathrm{~K}$ cold heat exchanger (CHX) and the JT restriction.

The full-scale $8 \mathrm{~K}$ helium JT cold stage demo was designed, fabricated and tested. It obtained $0.42 \mathrm{~W}$ cooling power at $8 \mathrm{~K}$ which is above the requirement $(0.4 \mathrm{~W})$ and within the $25 \%$ design margin.

\section{Scaled helium sorption compressor:}

This second demo is a scaled version of the helium compressor in the METIS cooler chain, which contains sorption compressor cells of sizes representative for METIS and operates with the same heat-sink temperature as the METIS cooler. However, the number of the cells is limited to about one tenth of the METIS helium compressor. The aim was to validate the performance of the helium sorption compressor, in particular regarding COP, cell size and the dynamic behavior of the sorption compressor. The setup was designed for four full size $(\varnothing 14.5 * 500 \mathrm{~mm})$ helium compressor cells, as an assembly expected to deliver a flow rate of $10.24 \mathrm{mg} / \mathrm{s}$ between 7.62 bar and 14.08 bar at a heat-sink temperature of $70 \mathrm{~K}$.

Since efficiency in this ground application was not of major concern, we developed a sorption compressor that could operate without a gas-gap heat switch. A dynamic model for this type of compressor was built and experimentally verified for simulating this new configuration and predicting the performance of the sorption compressor. Compared to the original configuration with a gas-gap heat switch, the switchless design requires $20 \%$ more input power but $14 \%$ fewer cells, and it greatly reduces manufacturing difficulties and production costs. Based on the model the detailed dimensions of the switchless compressor cells for the METIS cooler were determined.

Using the switchless configuration, the downscaled helium sorption compressor for the METIS cooler was designed, fabricated, assembled and tested. It consisted of 4 compressor cells connected in parallel with individual check valves. Two cells were made following the baseline cell design of which, unfortunately, one could not be used because of a heater defect. The other two cells have an enhanced radial conduction that was realized by inserting aluminum foil between the carbon pills. The baseline cell can produce a mass flow rate of $2.73 \mathrm{mg} / \mathrm{s}$ between 14.08 bar and 7.48 bar with a heat-sink temperature of $70 \mathrm{~K}$. The improvement by increasing the effective conductivity of the adsorbent material was verified: the other two cells performed better in the same setting, the mass flow being increased by $8.7 \%$ and $7.2 \%$.

Furthermore, the sorption compressor performance against the cycling time and the heat-sink temperature was investigated. As the cycle time is increased, the cell can cool down closer to the heat-sink temperature and thus more gas is adsorbed. As a result, the efficiency increases but because of the longer cycle time, the average mass-flow rate decreases. Thus, a trade-off can be made between efficiency and number of cells needed 
for a specific cooling power. In this process, the heat-sink temperature has a strong effect. The efficiency changes close to $6 \%$ per kelvin of change in heat-sink temperature. The 1D dynamic model described in Chapter 6 appears to simulate the compressor operation very well. Deviations between simulation and experimental results are measured of a few percent and can fully be accounted for by inaccuracies in model input parameters such as isotherm data, specific heat, and thermal conductivity. Despite the sensitivity to these input parameters, the model is very useful for design purposes. Finally, the compressor was operated with three cells running $120^{\circ}$ out of phase, and a total mass flow rate of $9.2 \mathrm{mg} / \mathrm{s}$ was achieved.

\section{Scaled $40 \mathrm{~K}$ neon sorption cooler:}

This is a complete neon-stage demo-cooler establishing about one fourth of the required cooling power of the neon stage cooler for METIS. The purpose is to demonstrate a fullyfunctional sorption cooler and validate the design of the critical components. It was designed to produce $1.0 \mathrm{~W}$ cooling power at $40 \mathrm{~K}$ with a neon flow rate of $35.6 \mathrm{mg} / \mathrm{s}$ and a heat-sink temperature of $70 \mathrm{~K}$.

This $40 \mathrm{~K}$ neon sorption JT cooler setup was designed and fabricated. The cold stage of this cooler was tested in an open loop, and $1.00 \mathrm{~W}$ at $40 \mathrm{~K}$ was achieved as expected. The orifice JT restriction was verified with an inaccuracy of $8 \%$ and the CFHX also performed according to the design. However, in future the $40 \mathrm{~K} \mathrm{CHX}$ has to be redesigned to increase the thermal contact between the evaporating neon and the cold plate and thus to reduce the temperature difference required for adequate heat transfer. The compressor cells for this neon cooler demo are under construction at Airbus Defence and Space Netherlands and, after completion, these will be combined with the cold stage for fullsystem test experiments.

\subsection{Outlook}

In order to have these sorption coolers applied in ground-based telescopes, their vibrationless operation in combination with an optical detector system should be demonstrated. Next, to make the technology even more competitive to traditional mechanical coolers, sorption coolers should be further improved with respect to efficiency and size. At the same time, the manufacturability, complexity of the system and the cost should be taken into account as well. To achieve this improvement, the following topics can be focused on in the future:

Adsorbent material: The adsorption capacity of the adsorbent will influence the sorption compressor performance significantly. Other adsorbent materials, such as metalorganic frameworks, may be suitable and have a better performance. For the saran carbon, its micro- and meso- structures and pore distribution can be further optimized for each particular working fluid.

Advanced adsorption cell design: To further improve the performance of the sorp- 
tion compressor, advanced adsorption cell design is required. Improving the heat transfer coefficient for heating/cooling the adsorbent is a key requirement for such design. Extending the heat transfer area, such as adding metal foam [89] or thin sheets (this thesis) in the adsorbent material, is one option. Distributive heating/cooling, which aims to deliver/extract heat more efficiently to and from the adsorbent material, is also very much of interest. Furthermore, advanced flow management is attractive to reduce the flow resistance for the working fluid entering the micro-pores of the adsorbent. Combining advanced additive manufacturing technique, it is possible to build composite micro-channels, such as fractal-shaped microchannel networks [90-92], in the adsorbent material. This is especially important for the low pressure operation when the loss associated with pressure drops becomes more significant.

Heat (cooling) Regeneration: Heat regeneration is a technique that is used in many adsorption heat-pump devices [93]. One sorption cell can be heated halfway by the waste heat that is recovered from other cells, to achieve an improvement in efficiency. This technique was also used in sorption coolers for cryogenic cooling [94]. For the METIS cooler, the sorption compressors are thermally cycled by electrical heating and cryogenic cooling from the $70 \mathrm{~K}$ heat sink that is established by pumped LN2. It is possible to use the regeneration technique to recover the cooling energy from cells to cool others. Therefore, it can greatly save LN2 and thus reduce the operation cost significantly.

Controlled heating: In this thesis, the heating of the sorption cell is constant in power. However, one can easily control the electrical heating power. To achieve a better performance, the heating power profile during the heating process of the compression cycle can be optimized to generate a uniform temperature profile in the adsorbent material and minimize the parasitic heat losses. Furthermore, the controlled heating can be used to stabilize the high pressure.

Heat switch: In Chapter 5, we propose the switchless sorption compressor concept for the METIS cooler to reduce manufacturing difficulties and production costs. The benefit of this modification was compared to the gas-gap heat switch concept that was used in previous work for space application. However, there are many alternative heat-switch types that may be suitable for the sorption compressor operating at cryogenic temperature $[95,96]$, such as a piezoelectric heat switch, a superconducting heat switch, a differential thermal expansion heat switch, etc.. The feasibility of these heat-switch types for ground applications like the METIS cooler needs to be further investigated.

Thermoelectric device: Gordon, etc. [97] proposed a miniature refrigerator that combined adsorption and thermoelectric devices. The idea is to use thermoelectric device to regenerate heat between sorption cells in an adsorption refrigerator.

Alternatively, for the METIS cooler thermoelectric devices can be assembled between the sorption container and the heat sink as a heat switch, as shown in Figure 8.1. During the heating process of the compression cycle, the thermoelectric device operates as a heat pump to pump heat from the heat sink to the adsorbent material. In such a way, the heat conduction loss during the heating phase is eliminated. In addition, compared to the 


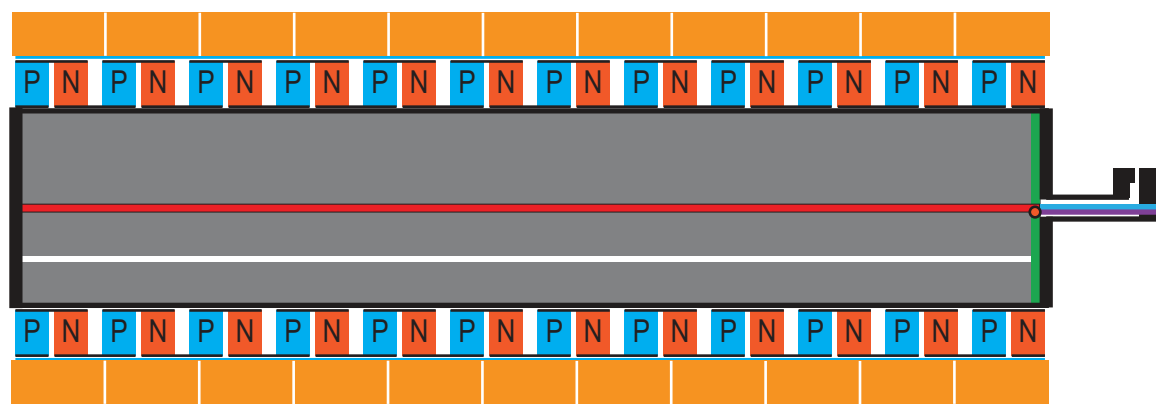

Figure 8.1. METIS sorption compressor combined with thermoelectric device.

central electrical heating, the heating effect of the thermoelectric device from the outer surface of the sorption container will establish a more uniform temperature distribution in the adsorbent. When the cell is cooled, the supply current for the thermoelectric device is reversed. The thermoelectric device then pumps heat from the adsorbent material to the heat sink. Such active heat pumping can greatly accelerate the cooling process resulting a faster cycling and reduction in the required number of cells. 


\section{Convection Term Approximation in 1-D Sorption Compressor Model}

The mass and energy conservation are considered in the 1-D sorption compressor model that described in Chapter 5, whereas the momentum equation is neglected. Therefore an approximation on the convection term in the energy equation is made, in which the velocity dependency is neglected. This appendix explains how this approximation is made. 
In the 1-D model for the sorption compressor described in Chapter 5, the energy conservation of the adsorbent mixture is given by Eq. 5.5,

$$
\begin{aligned}
& \rho_{\text {sor, },}[\underbrace{\left(c_{\text {sor }}+x_{a d s} c_{a d s}+x_{f r e} c_{p, f r e}\right) \frac{\partial T}{\partial t}}_{\text {(a.1) sensible heat }}-\underbrace{\left.\frac{R T^{2}}{M p} \frac{\partial p}{\partial T}\right|_{x_{a d s}} \frac{\partial x_{a d s}}{\partial t}}_{\text {(a.2) heat of adsorption }}]= \\
& \text { (a) Unsteady term (enthalpy change) } \\
& \underbrace{\nabla \cdot\left(k_{e f f} \nabla T\right)}-\underbrace{\rho_{\text {sor }, b} \nabla \cdot\left(x_{\text {fre }} h_{\text {fre }} \vec{V}\right)} \\
& \begin{array}{ll}
\text { (b) Diffusion term } & \text { (c) Convection term }
\end{array} \\
& \text { (heat flow due to the conduction) (enthalpy flow due to the convection) }
\end{aligned}
$$

The second term on the right side of the equation is the convection term that indicates the enthalpy flow due to the mass redistribution of the free working fluid. The velocity field as such of the fluid in the cell is not of direct interest to the model. In addition, we do not know its magnitude and, therefore, we will reformulate this second term such that the velocity disappears from the equation and is replaced by known parameters.

The convection term can be rewritten as,

$$
\rho_{\text {sor }, b} \nabla \cdot\left(x_{\text {fre }} h_{\text {fre }} \vec{V}\right)=\rho_{\text {sor }, b} h_{\text {fre }}\left(\nabla \cdot\left(x_{\text {fre }} \vec{V}\right)\right)+\rho_{\text {sor }, b} x_{\text {fre }} \vec{V} \cdot \nabla\left(h_{\text {fre }}\right)
$$

Using the mass conservation equation,

$$
\frac{\partial x_{t o t}}{\partial t}+\nabla \cdot\left(x_{f r e} \vec{V}\right)=0
$$

the convection term can be expressed as,

$$
\rho_{\text {sor }, b} \nabla \cdot\left(x_{\text {fre }} h_{f r e} \vec{V}\right)=-\rho_{\text {sor }, b} h_{\text {fre }} \frac{\partial x_{\text {tot }}}{\partial t}+\rho_{\text {sor }, b} x_{f r e} \vec{V} \cdot \nabla\left(h_{\text {fre }}\right)
$$

In the expression of the convection term, the velocity only appears in the second term that represents the effect of the gradient of the free fluid specific enthalpy in the velocity direction.

During the compression and decompression phases, the check valves are closed. According to the symmetric geometry of the adsorbent mixture domain, one can consider the free fluid to transfer only in the radial direction. By such assumption, the second term at the right side of Eq. A.4 can evaluated. It is a few percentage of the unsteady term. Therefore, it is reasonable to neglect this term in the 1-D model to simplify the calculation.

In the out-flow phase, the high-pressure check valve is open allowing for a flow out of the sorption compressor. Here, we assume that the radial fluid transfer is negligible and flow only occurs in axial direction. Thus, $\nabla\left(h_{f r e}\right)$ represents the gradient in the specific enthalpy of the working fluid at the flow boundary. At the closed end of the cell the 
flow is zero and at the open end the enthalpy of the fluid does not change (temperature is constant). Therefore, again the second term at the right side of Eq. A.4 can be neglected.

Similarly, the radial fluid transfer is neglected for the in-flow phase. In this, phase, the flow into the compressor cell comes from the low-pressure side of the cold stage with a pressure of $p_{l}$ and a temperature of the heat-sink temperature, $T_{H S}$. Therefore, $\nabla\left(h_{f r e}\right)$ can be approximated by,

$$
\nabla\left(h_{f r e}\right) \approx \frac{\left(h_{f r e}-h_{l, H S}\right)}{\Delta z}
$$

Here, $h_{l, H S}$ is the specific enthalpy of the working fluid at pressure $p_{l}$ and temperature $T_{H S}$, and $h_{f r e}$ is the specific enthalpy of the free working fluid in the cell. Furthermore, $\Delta z$ is an infinite small length at the flow boundary in which the incoming cold flow is decelerated to zero and heated to the temperature of the adsorbent mixture. The second term at the right side of Eq. A.4 can now be written as,

$$
\begin{aligned}
\rho_{s o r, b} x_{f r e} \vec{V} \cdot \nabla\left(h_{f r e}\right) & \approx \rho_{s o r, b} x_{f r e} V \frac{\left(h_{f r e}-h_{l, H S}\right)}{\Delta z} \\
& \approx \rho_{s o r, b}\left(h_{f r e}-h_{l, H S}\right) \frac{\partial x_{t o t}}{\partial t}
\end{aligned}
$$

Substitution of Eq. A.6 into Eq. A.4 results in,

$$
\rho_{\text {sor }, b} \nabla \cdot\left(x_{\text {fre }} h_{f r e} \vec{V}\right) \approx-\rho_{\text {sor }, b} h_{l, H S} \frac{\partial x_{\text {tot }}}{\partial t}
$$

In summary, the convection term in Eq. 5.5 is approximately calculated by,

$$
\rho_{s o r, b} \nabla \cdot\left(x_{f r e} h_{f r e} \vec{V}\right) \approx \begin{cases}-\rho_{s o r, b} h_{l, H S} \frac{\partial x_{t o t}}{\partial t} & \text { in-flow phase } \\ -\rho_{s o r, b} h_{f r e} \frac{\partial x_{t o t}}{\partial t} & \text { other phases }\end{cases}
$$





\section{Bibliography}

[1] R. Gilmozzi and P. Dierickx, "OWL Concept Study," The Messenger, vol. 100, pp. 1-10, 2000.

[2] G. Monnet and R. Gilmozzi, "Status of the European ELT," The Messenger, vol. 123, pp. 4-5, 2006.

[3] “OWL Concept Design Report,” European Southern Observatory, Tech. Rep. OWLTRE-ESO-0000-0001, 2004.

[4] The E-ELT Construction Proposal. München, Germany: European Southern Observatory, 2011.

[5] E-ELT Site Chosen, http://www. eso.org/public/news/eso1018/, 2010.

[6] R. Gilmozzi and J. Spyromilio, "The European Extremely Large Telescope (EELT)," The Messenger, vol. 127, pp. 11-19, 2007.

[7] B. R. Brandl, R. Lenzen, E. Pantin, et al., "Instrument concept and science case for the mid-IR E-ELT imager and spectrograph METIS," in Proc. SPIE 7735, Groundbased and Airborne Instrumentation for Astronomy III, I. S. McLean, S. K. Ramsay, and H. Takami, eds., 2010, 77352G.

[8] B. R. Brandl, R. Lenzen, E. Pantin, et al., "METIS: the thermal infrared instrument for the E-ELT," in Proc. SPIE 8446, Ground-based and Airborne Instrumentation for Astronomy IV, I. S. McLean, S. K. Ramsay, and H. Takami, eds., 2012, p. $84461 \mathrm{M}$.

[9] G. Kroes, R. ter Horst, J. Kragt, et al., "METIS opto-mechanical design and packaging study," in Proc. SPIE 7735, Ground-based and Airborne Instrumentation for Astronomy III, I. S. McLean, S. K. Ramsay, and H. Takami, eds., 2010, 77352J. 
[10] B. Benthem, J. Doornink, E. Boom, H. J. Holland, P. P. P. M. Lerou, J. F. Burger, and H. J. M. ter Brake, "Present status of developments in physical sorption cooling for space applications," Cryogenics, vol. 64, pp. 220-227, 2014.

[11] J. M. F. Vickers and E. J. Romvary, Intermittent type silica gel adsorption refrigerator, US Patent: 3270512A, 1963.

[12] H. H. van Mal and A. Mijnheer, "Hydrogen refrigerator for the $20 \mathrm{~K}$ region with a LaNi5-hydride thermal absorption compressor for hydrogen," in Proceedings of $4^{\text {th }}$ International Cryogenic Engineering, vol. 4, IPC Science and Technology Press Ltd., 1972, pp. 122-125.

[13] W. H. Hartwig, “Adsorption pumping cryogenic refrigerator studies," Engineering of Science, University of Texas at Austin, 47th Monthly Progress Report, National Aeronautics and Space Administration, Lyndon B. Johnson Space Center, Dec. 1, 1978-Dec. 31, Contract No. NASA 9-14491, Tech. Rep., 1978.

[14] W. H. Hartwig, "Cryogenic Refrigeration Concepts Utilizing Adsorption Pumping in Zeolites," in Advances in Cryogenic Engineering 23, K. D. Timmerhaus, ed., New York, US: Plenum Press, 1978, pp. 438-447.

[15] W. H. Hartwig, A. W. Woltman, and J. P. Masson, "Design theory and performance of cryogenic molecular adsorption refrigeration system," in Proceedings of $7^{\text {th }}$ International Cryogenic Engineering Conference, IPC Science and Technology Press Ltd., 1978, pp. 409-414.

[16] J. A. Jones, "LaNi ${ }_{5}$ hydride cryogenic refrigerator test results," in Refrigeration of Cryogenic Sensors, M. Gasser, ed., NASA, 1983, pp. 357-373.

[17] J. A. Jones, "Hydride adsorption refrigerator system for ten kelvin and below," in Proceedings of the Third Cryocooler Conference, 1984, pp. 53-64.

[18] K Karperos, "Operating characteristics of a hydrogen sorption refrigerator part 1: experiment design and results," in $4^{\text {th }}$ Proc. International Cryocooler Conference, G. Green, G. Patton, and M. Knox, eds., 1986, pp. 1-15.

[19] S. Bard, "Development of an 80-120 K charcoal-nitrogen adsorption cryocooler," in $4^{\text {th }}$ Proc. International Cryocooler Conference, G. Green, G. Patton, and M. Knox, eds., 1986, pp. 43-56.

[20] J. A. Jones, "Sorption cryogenic refrigeration - status and future," in Advances in Cryogenic Engineering 33, R. W. Fast, ed., New York, US: Plenum Press, 1988, pp. 869-878.

[21] S. Bard and J. A. Jones, "Development and Testing of an 80 K Oxide Sorption Cryocooler," in $5^{\text {th }}$ Proc. International Cryocooler Conference, 1988, pp. 13-23. 
[22] J. Alvares, E. Ryba, P. Sywulka, and L. A. Wade, "Design and Component Test Performance of an Efficient 4 W, 130 K Sorption Refrigerator," in Advances in Cryogenic Engineering 35B, R. W. Fast, ed., New York, US: Plenum Press, 1990, pp. 1367-1374.

[23] J. A. Jones and A. Lund, "Sorption J-T refrigeration utilizing manganese nitride chemisorption," in Advances in Cryogenic Engineering 35B, R. W. Fast, ed., New York, US: Plenum Press, 1990, pp. 1383-1390.

[24] L. Wade, E. Ryba, C. Weston, and J. Alvarez, "Test performance of a 2 W, $137 \mathrm{~K}$ sorption refrigerator," Cryogenics, vol. 32, no. 2, pp. 122-126, 1992.

[25] J. A. Alvares, R. J. Krylo, R. D. Snapp, C. Weston, P. Sywulka, and G. C. Abell, "Development of an Advanced Sorption Compressor and Its Application in a 125 K Cryocooler," in Cryocoolers 8, R. G. J. Ross, ed., New York, US: Plenum Press, 1995, pp. 569-579.

[26] L. A. Wade, J. J. Wu, S. Bard, T. B. Flanagan, J. D. Clewley, and S. Lou, "Performance, reliability, and life of hydride compressor components for 10 to $30 \mathrm{~K}$ sorption cryocoolers," in Advances in Cryogenic Engineering 39B, P. Kittle, ed., New York, US: Plenum Press, 1994, pp. 1483-1490.

[27] R. C. J. Bowman, D. R. Gilkinson, R. D. Snapp, G. C. Abell, B. D. Freeman, and E. L. Ryba, "Fabrication and testing of the metal hydride sorbent bed assembly for a periodic $10 \mathrm{~K}$ sorption cooler," in Cryocoolers 8, R. G. J. Ross, ed., New York, US: Plenum Press, 1995, pp. 601-608.

[28] S. Bard, J. J. Wu, P. Karlmann, P. Cowgill, C. Mirate, and J. Rodrigues, "Ground testing of a $10 \mathrm{~K}$ sorption cryocooler flight experiment (BETSCE)," in Cryocoolers 8, R. G. J. Ross, ed., New York, US: Plenum Press, 1995, pp. 609-621.

[29] S. Bard, P. Karlmann, J. Rodrigues, J. Wu, L. Wade, P. Cowgill, and K. M. Russ, "Flight demonstration of a 10 K sorption cooler," in Cryocoolers 9, R. G. J. Ross, ed., New York, US: Plenum Press, 1997, pp. 567-576.

[30] J. J. Wu, S. Bard, W. Boulter, J. Rodrigues, and R. Longsworth, "Experimental Demonstration of a $10 \mathrm{~K}$ Sorption Cryocooler Stage," in Advances in Cryogenic Engineering 39B, P. Kittle, ed., New York, US: Plenum Press, 1994, pp. 15071514.

[31] P. Bhandari, M. Prina, R. C. Bowman Jr, C. Paine, D. Pearson, and A. Nash, "Sorption coolers using a continuous cycle to produce $20 \mathrm{~K}$ for the Planck flight mission," Cryogenics, vol. 44, no. 6-8, pp. 395-401, 2004.

[32] R. C. Bowman, M. Prina, D. S. Barber, et al., "Evaluation of hydride compressor elements for the Planck sorption cryocooler," in Cryocoolers 12, R. G. Ross, ed., New York, US: Kluwer Acadamic / Plunum Publishers, 2003, pp. 627-635. 
[33] Last Command sent to ESA's Planck Space Telescope, http : / /www . esa . int / Our_Activities / Space_Science / Planck / Last_command_sent_to_ESA_S_ Planck_space_telescope, 2013.

[34] J. F. Burger, "Cryogenic microcooling," PhD thesis, University of Twente, Enschede, The Netherlands.

[35] J. F. Burger, H. J. Holland, J. H. Seppenwoolde, et al., "165 K Microcooler Operating with a Sorption Compressor and a Micromachined Cold Stage," in Cryocoolers 11, R. G. J. Ross, ed., New York, US: Kluwer Academic / Plunum Publishers, 2001, pp. 551-560.

[36] G. F. M. Wiegerinck, "Improving sorption compressors for cryogenic cooling," $\mathrm{PhD}$ thesis, University of Twente, Enschede, The Netherlands, 2005.

[37] G. F. M. Wiegerinck, J. F. Burger, H. J. Holland, E. Hondebrink, H. J. M. ter Brake, and H. Rogalla, "A sorption compressor with a single sorber bed for use with a Linde-Hampson cold stage," Cryogenics, vol. 46, no. 1, pp. 9-20, 2006.

[38] G. F. M. Wiegerinck, H. J. M. ter Brake, J. F. Burger, H. J. Holland, and H. Rogalla, "Thermodynamic optimization of sorption-based Joule-Thomson coolers," Cryogenics, vol. 47, no. 3, pp. 143-152, 2007.

[39] J. F. Burger, H. J. M. ter Brake, H. Rogalla, and M. Linder, "Vibration-free $5 \mathrm{~K}$ sorption cooler for ESA's Darwin mission,” Cryogenics, vol. 42, no. 2, pp. 97-108, 2002.

[40] J. F. Burger, H. J. M. ter Brake, H. J. Holland, et al., "Long-life vibration-free 4.5 K sorption cooler for space applications," Review of Scientific Instruments, vol. 78, no. 6, pp. 065 102-10, 2007.

[41] H. J. M. ter Brake, J. F. Burger, H. J. Holland, R. J. Meijer, A. V. Mudaliar, D. Zalewski, and M. Linder, “14.5 K Hydrogen Sorption Cooler: Design and Breadboard Tests,” in Cryocoolers 16, S. D. Miller and R. G. J. Ross, eds., Boulder, CO, USA: ICC Press, 2009, pp. 445-454.

[42] H. J. M. ter Brake, "Cryogenic Systems for Superconducting Devices," in Applications of Superconductivity, H. Weinstock, ed., ser. NATO ASI Series. Dordrecht, The Netherlands: Kluwer Academic Publishers, 2000, vol. 365, ch. 13.

[43] T. L. Bergman, A. S. Lavine, F. P. Incropera, and D. P. DeWitt, Fundamentals of Heat and Mass Transfer, 7th ed. John Wiley \& Sons, 2011.

[44] G. Walker, Cryocoolers. New York: Plenum Press, 1983.

[45] L. A. Wade, "An Overview of the Development of Sorption Refrigeration," in Advances in Cryogenic Engineering 37B, R. W. Fast, ed., New York: Plenum Press, 1992, pp. 1095-1106.

[46] S. Bard, "Improving adsorption cryocoolers by multi-stage compression and reducing void volume," Cryogenics, vol. 26, no. 8, pp. 450-458, 1986. 
[47] E. Robens, "Some intriguing items in history of adsorption," in Characterization of Porous Solids III, J. Rouquerol, F. Rodriguez-Reinoso, K. S. W. Sing, and K. K. Unger, eds. Amsterdam: Elsevier Science, 1994, pp. 109-118.

[48] F. Rouquerol, J. Rouquerol, and K. Sing, Adsorption by Powders and Porous Solids: Principles, Methodology and Applications. London, UK: Academic Press, 1999.

[49] L. Zhou, "Adsorption Isotherms for the Supercritical Region," in Adsorption: Theory, Modeling, and Analysis, J. Tóth, ed. New York, US: Marcel Dekker, Inc., 2002, pp. 211-250.

[50] L. Zhou, Y. Zhou, and Y. Sun, “A comparative study of hydrogen adsorption on superactivated carbon versus carbon nanotubes," International Journal of Hydrogen Energy, vol. 29, no. 5, pp. 475-479, 2004.

[51] A. Chambers, C. Park, R. T. K. Baker, and N. M. Rodriguez, "Hydrogen Storage in Graphite Nanofibers," The Journal of Physical Chemistry B, vol. 102, no. 22, pp. 4253-4256, 1998.

[52] J. P. Redmond and P. L. Walker, "Hydrogen sorption on graphite at elevated temperatures," The Journal of Physical Chemistry, vol. 64, no. 9, pp. 1093-1099, 1960.

[53] M. A. Ban̄ares-Mun̄oz, L. V. F. González, and J. M. M. Llorenta, “Adsorption isotherms of nitrogen and argon on an "agot" grade artificial nuclear graphite at 77 and 90 K," Carbon, vol. 25, no. 5, pp. 603-608, 1987.

[54] H. L. McDermot and J. C. Arnell, "The adsorption of nitrogen, oxygen, and argon by graphite," Canadian Journal of Chemistry, vol. 33, no. 5, pp. 913-922, 1955.

[55] J. F. Wacker, "Laboratory simulation of meteoritic noble gases. iii. sorption of neon, argon, krypton, and xenon on carbon: elemental fractionation," Geochimica et Cosmochimica Acta, vol. 53, no. 6, pp. 1421-1433, 1989.

[56] M. Polanyi, "Section III.-Theories of the adsorption of gases. A general survey and some additional remarks," Transactions of the Faraday Society, vol. 28, pp. 316333, 1932.

[57] M. M. Dubinin, "The potential theory of adsorption of gases and vapors for adsorbents with energetically nonuniform surfacesu," Chemical Reviews, vol. 60, no. 2, pp. 235-241, 1960.

[58] S. Ozawa, S. Kusumi, and Y. Ogino, "Physical Adsorption of Gases at High pressure - IV. An Improvement of the Dubinin-Astakhov Adsorption Equation," Journal of Colloid and Interface Science, vol. 56, no. 1, pp. 83-91, 1976.

[59] R. K. Agarwal and J. A. Schwarz, "Analysis of High-Pressure Adsorption of Gases on Activated Carbon by Potential-Theory," Carbon, vol. 26, no. 6, pp. 873-887, 1988. 
[60] M. M. Dubinin, "Physical Adsorption of Gases and Vapors in Micropores," in Progress in Surface and Membrane Science, D. A. Cadenhead, J. F. Danielli, and M. D. Rosenberg, eds. New York: Academic Press, 1975, vol. 9, ch. 1, pp. 1-70.

[61] D. M. Ruthven, Principles of adsorption and adsorption processes. New York, USA: John Wiley \& Sons, 1984.

[62] M. Suzuki, Adsorption Engineering. Amsterdam: Elsevier Science Publishers, 1990.

[63] K. S. Walton and M. D. LeVan, “Adsorbed-Phase Heat Capacities: Thermodynamically Consistent Values Determined from Temperature-Dependent Equilibrium Models," Industrial E Engineering Chemistry Research, vol. 44, no. 1, pp. 178182, 2005.

[64] J. F. Burger, H. J. Holland, L. A. Wade, H. J. M. ter Brake, and H. Rogalla, "Thermodynamic Considerations on a Microminiature Sorption Cooler," in Cryocoolers 10, R. G. J. Ross, ed., New York, US: Kluwer Academic / Plenmu Publishers, 1999, pp. 553-563.

[65] J. H. Derking, H. J. M. ter Brake, A. Sirbi, M. Linder, and H. Rogalla, "Optimization of the working fluid in a Joule-Thomson cold stage," Cryogenics, vol. 49, no. 3-4, pp. 151-157, 2009.

[66] E. W. Lemmon, M. L. Huber, and M. O. McLinden, Refprop 8.0, National Institute of Standards and Technology, 2007.

[67] V. C. Menon and S. Komarneni, "Porous adsorbents for vehicular natural gas storage: a review," Journal of Porous Materials, vol. 5, no. 1, pp. 43-58, 1998.

[68] A. Yavrouian and H. Schember, "Saran carbon sorbent development for sorption cryocooler use," Jet Propulsion Laboratory, California Institute of Technology, Tech. Rep. D-7368, 1990.

[69] T. Otowa, R. Tanibata, and M. Itoh, "Production and adsorption characteristics of MAXSORB: High-surface-area active carbon," Gas Separation $\mathcal{F}$ Purification, vol. 7, no. 4, pp. 241-245, 1993.

[70] J. F. Burger, H. J. Holland, R. J. Meijer, A. Sirbi, and H. J. M. ter Brake, "Vibration free 4.5 K sorption cooler," in Advances in Cryogenic Engineering 53B, J. G. Weisend II, ed., Melville, New York: AIP, 2008, pp. 1613-1620.

[71] H. J. M. ter Brake, Y. Wu, D. R. Zalewski, et al., "Sorption-based vibration-free cooler for the METIS instrument on E-ELT," in Proc. SPIE 8446, Ground-based and Airborne Instrumentation for Astronomy IV, I. S. McLean, S. K. Ramsay, and H. Takami, eds., 2012, 84467O.

[72] J. Nocedal and S. J. Wright, Numerical Optimization, 2nd ed., ser. Springer Series in Operations Research and Financial Engineering. New York: Springer, 2006. 
[73] D. E. Knuth, The art of computer programming, Volume 3: Sorting and Searching, 2nd ed. Redwood City, CA, USA: Addison Wesley Longman Publishing Co., Inc., 1998.

[74] B.-Z. Maytal and J. M. Pfotenhauer, Miniature Joule-Thomson Cryocooling: Principles and Practice, ser. International Cryogenics Monograph Series. Springer New York, 2013.

[75] H. Quack, "Theory of cascade refrigeration," in Advances in Cryogenic Engineering 57A, J. G. Weisend II, ed., Melville, New York: AIP, 2012, pp. 793-789.

[76] K. D. Timmerhaus and T. M. Flynn, Cryogenic Process Engineering. New York: Springer US, 1989.

[77] A. P. Rijpma and H. J. M. ter Brake, "Cryogenic thermometry with a common diode: Type BAS16," Cryogenics, vol. 46, no. 1, pp. 68-69, 2006.

[78] R. K. Shah and D. P. Sekulić, Fundamentals of Heat Exchanger Design. Hoboken, NJ, USA: John Wiley \& Sons, Inc., 2003.

[79] C. K. Chan, "Improved heat switch for gas sorption compressor," in Proceedings of the Third Cryocooler Conference, R. Radebaugh, B. Louie, and S. McCarthy, eds., Boulder, CO, USA: National Bureau of Standards, 1985, pp. 42-52.

[80] J. F. Burger, H. J. Holland, H. van Egmond, M. Elwenspoek, H. J. M. ter Brake, and H. Rogalla, "Fast Gas-Gap Heat Switch for a Microcooler," in Cryocoolers 10, R. G. J. Ross, ed., New York, US: Kluwer Academic / Plenmu Publishers, 1999, pp. 565-574.

[81] M. Prina, P. Bhandari, R. C. Bowman, C. Paine, and L. A. Wade, "Development of gas gap heat switch actuator for the PLANCK sorption cryocooler," in Advances in Cryogenic Engineering 45A, Q. Shu, ed., New York: Kluwer Academic/Plenum Publishers, 2000, pp. 553-560.

[82] J. W. Ekin, Experimental Techniques for Low-Temperature Measurements: Cryostat Design, Material Properties and Superconductor Critical-Current Testing. Oxford, UK: Oxford University Press, 2006.

[83] Y. Wu, T. Mulder, C. H. Vermeer, H. J. Holland, B. Benthem, and H. J. M. ter Brake, "Switchless sorption-compressor design," in Physics Procedia vol.67: Proceedings of $25^{\text {th }}$ International Cryogenic Engineering Conference, H. J. M. ter Brak, H. H. J. ten Kate, and S. Vanapalli, eds., 2014, pp. 411-416.

[84] T. Mulder, "New sorption compressor designs for the METIS cooler in the E-ELT," Master's thesis, University of Twente, Enschede, The Netherlands, 2014.

[85] E. D. Marquardt, J. P. Le, and R. Radebaugh, "Cryogenic Material Properties Database," in Cryocoolers 11, R. G. J. Ross, ed., New York, US: Kluwer Acadamic / Plunum Publishers, 2001, pp. 681-687. 
[86] T. T. Veenstra, G. C. F. Venhorst, J. F. Burger, H. J. Holland, H. J. M. ter Brake, A. Sirbi, and H. Rogalla, "Development of a stainless steel check valve for cryogenic applications,” Cryogenics, vol. 47, no. 2, pp. 121-126, 2007.

[87] B.-Z. Maytal, "Real gas choked flow conditions at low reduced-temperatures," Cryogenics, vol. 46, no. 1, pp. 21-29, 2006.

[88] J. M. Astruc, A. Lacaze, and P. Perroud, "Comparison of heat transfer to hydrogen, deuterium, and neon boiling with free convection at atmospheric pressure," Cryogenics, vol. 9, no. 4, pp. 248-250, 1969.

[89] P. Bhandari, R. C. Bowman, R. G. Chave, et al., "Sorption cryocooler development for the Planck surveyor mission," Astrophysical Letters and Communications, vol. 37, pp. 227-237, 2000.

[90] D. V. Pence, "Reduced pumping power and wall temperature in microchannel heat sinks with fractal-like branching channel networks," Microscale Thermophysical Engineering, vol. 6, no. 4, pp. 319-330, 2003.

[91] Y. P. Chen and P. Cheng, "Heat transfer and pressure drop in fractal tree-like microchannel nets," International Journal of Heat and Mass Transfer, vol. 45, no. 13, pp. 2643-2648, 2002.

[92] W. Escher, B. Michel, and D. Poulikakos, "Efficiency of optimized bifurcating treelike and parallel microchannel networks in the cooling of electronics," International Journal of Heat and Mass Transfer, vol. 52, no. 5-6, pp. 1421-1430, 2009.

[93] M. Pons, "Analysis of the adsorption cycles with thermal regeneration based on the entropic mean temperatures," Applied Thermal Engineering, vol. 17, no. 7, pp. 615-627, 1997.

[94] S. Bard and J. A. Jones, "Regenerative sorption compressors for cryogenic refrigeration," in Advances in Cryogenic Engineering 35B, R. W. Fast, ed., New York, US: Plenum Press, 1990, pp. 1357-1365.

[95] M. J. DiPirro and J. Shirron P, "Heat switches for ADRs," Cryogenics, vol. 62, pp. 172-176, 2014.

[96] D. G. Gilmore, Spacecraft Thermal Control Handbook: Fundamental technologies. Aerospace Press, 2002.

[97] J. M. Gordon, K. C. Ng, H. T. Chua, and A. Chakraborty, "The electro-adsorption chiller: a miniaturized cooling cycle with applications to micro-electronics," International Journal of Refrigeration, vol. 25, no. 8, pp. 1025-1033, 2002. 


\section{Summary}

METIS, the Mid-infrared E-ELT Imager and Spectrograph, is one of instruments in the European Extremely Large Telescope. Its detectors require cryogenic cooling at three temperature levels below that of liquid nitrogen, $8 \mathrm{~K}, 25 \mathrm{~K}$, and $40 \mathrm{~K}$. Vibration-free cooling is one of the technologies that were identified as the most needed in the development of the METIS instruments. Therefore, a useful cooling technology based on sorption Joule-Thomson (JT) coolers was proposed for the METIS by University of Twente. Sorption-based JT coolers operate vibration-free, have a potentially long life time, and cause no electromagnetic interference. So they are appealing to a wide variety of applications, such as cooling of low-noise amplifiers, superconducting electronics, and optical detectors.

This research focuses on developing a vibration-free sorption-based JT cooler for the METIS instrument in the E-ELT. The research is characterized by challenges such as multiple cooling levels, large cooling capacity with considerable efficiency and size, manufacturability and costs, etc.. The development was carried out following a roadmap:

\section{Initialization:}

The fundamental principles related to the sorption cooler technology were gathered and studied, as well as the basic inputs (e.g. the fluid properties; specifications, and characterizations of the adsorbent materials; and the cryogenic properties of the materials). Based on the properties of the working fluid and the adsorbent, we developed a generalized methodology for the optimization of the working fluid in a sorption-based JT cooler. This method was used to determine the most suitable working fluid for the specific cooling temperature levels in the METIS cooler chain. Furthermore, we modified a sophisticated adsorption-isotherm measuring setup to improve its measuring accuracy. A large number of adsorption isotherms was measured as input data for the sorption compressor develop- 
ment.

\section{Baseline design:}

In this level, a conceptual baseline design of the METIS cooler chain was raised to present a first impression. Three stages are thermally linked in parallel and provide cooling at $8 \mathrm{~K}$ (helium), $25 \mathrm{~K}$ (hydrogen) and $40 \mathrm{~K}$ (neon). The heat-sink temperature for the sorption compressors is at $70 \mathrm{~K}$. A quasi-static model was built. By using this model combined with suitable design margins and assumptions, we optimized the operating conditions (e.g. high pressures, regeneration temperatures and heat-sink temperature) and estimate the performance of the cooler chain on system level. Based on this baseline design, we identified the helium stage as the most critical one and we, therefore, focused research on that stage.

\section{Submodules design and validation:}

Following the baseline design of the METIS cooler chain, the work at this level was dedicated to realize two critical submodules in the cooler chain: (a) the helium JT cold stage and (b) the helium sorption compressor.

(a) The helium JT cold stage:

The helium JT cold stage consists of counter flow heat exchangers, JT restriction and $8 \mathrm{~K}$ cold heat exchanger. Numerical models were developed to design and optimize those components. A full-scale $8 \mathrm{~K}$ helium JT cold stage was fabricated and tested for demonstrating the helium JT cold stage in the METIS cooler chain. In this setup, a Gifford-McMahon cooler was used to provide the required pre-cooling power, whereas the cold stage was driven by a mechanical compressor. The measurement showed that this JT cold stage can obtain $0.42 \mathrm{~W}$ cooling power at $8.0 \mathrm{~K}$ which satisfies the design requirement $(0.4 \mathrm{~W})$.

(b) The helium sorption compressor:

The helium sorption compressor of the METIS cooler was validated by a scaled version of the helium compressor. The aim of this demo setup was to validate the performance of the helium sorption compressor, in particular regarding the coefficient of performance, cell size and the dynamic behavior of the sorption compressor. We developed a new configuration that allows the sorption compressor to operate without a gas-gap heat switch. This switchless design requires about $20 \%$ more input power but it uses $14 \%$ fewer cells and skipping the gas-gap heat switch greatly reduces manufacturing difficulties and production costs. A 1-dimensional dynamic model based on MATLAB for simulating the behavior of the sorption compressor and predicting its performance, was developed and experimentally validated. Based on that, the demo setup was designed in detail, fabricated, assembled and finally tested. In the baseline design, a compressor cell produced a mass flow rate of $2.73 \mathrm{mg} / \mathrm{s}$ with a heat-sink temperature of $70 \mathrm{~K}$. Incorporating enhanced radial thermal conduction by insertion of aluminum 
foil between the carbon pills, resulted in an increase of the mass flow rate 7 to $9 \%$ more than the baseline cell. The 1-dimensional dynamic sorption compressor model appears to simulate the compressor operation very well. Deviations between simulation and experimental results were measured of a few percent and can fully be accounted for by inaccuracies in model input parameters such as isotherm data, specific heat, and thermal conductivity. Despite the sensitivity to these input parameters, the model is very useful for design purposes. Finally, the compressor was operated with three cells running 120 out of phase, and a total mass flow rate of $9.2 \mathrm{mg} / \mathrm{s}$ was achieved.

\section{Full cooler demonstration:}

Associated with Airbus Defence \& Space Netherlands, a complete $40 \mathrm{~K}$ neon-stage demo cooler is under development to present the feasibility of the sorption cooling technology for this particular large-scale ground application. The $40 \mathrm{~K}$ JT cold stage was designed, fabricated and tested. A cooling power of $1.00 \mathrm{~W}$ at $40 \mathrm{~K}$ was achieved as expected. We also designed the neon sorption compressor unit and, at the moment of this writing, it is under construction at Airbus Defence \& Space Netherlands. After completion, the sorption compressor will be combined with the cold stage for full-cooler test experiments.

\section{Outlook:}

To make the technology even more competitive to traditional mechanical coolers, sorption coolers should be further improved with respect to efficiency and compactness. At the same time, the manufacturability, complexity of the system and the cost should be taken into account as well. To achieve such improvement, the following topics can be focused on in the future: improving the adsorption amount of the working fluid on the adsorbent by further optimizing the micro/meso structure and pore distribution in the adsorbent; looking for new adsorbent, such as metal-organic frameworks; developing a 3-dimensional sorption compressor model for further understanding the heat and mass transfer phenomena in the sorption compressor; improving the efficiency of heat transfer for heating/cooling the adsorbent and reducing pressure drops by introducing advanced sorption cell designs; optimizing a controlled heating; combining the heat(cooling) regeneration; seeking better candidates or alternatives for the heat switch device. 



\section{Samenvatting}

METIS is een mid-infrarood camera en spectrometer, (Mid-infrared E-ELT Imager and Spectograph) en is één van de instrumenten in E-ELT, de European Extremely Large Telescope. De detectoren in dit instrument hebben drie temperatuurniveaus nodig die beneden de temperatuur van vloeibaar stikstof liggen, namelijk: $8 \mathrm{~K}, 25 \mathrm{~K}$ en $40 \mathrm{~K}$. Eén van de technologieën die binnen het METIS instrument is geïdentificeerd als noodzakelijke ontwikkelingsstap, is trillingsvrije koeling. In dit verband heeft de Universiteit Twente op sorptie gebaseerde Joule Thomson (JT) koelers voorgesteld als geschikte koelertechnologie. Deze koelers functioneren trillingsvrij, kunnen een lange levensduur hebben, en veroorzaken geen storende elektromagnetische velden. Daarom zijn ze aantrekkelijk in een groot aantal toepassingsvelden zoals; koeling van ruisarme versterkers, supergeleidende elektronica en optische detectoren.

In dit promotie-onderzoek ligt de nadruk op het ontwikkelen van een trillingsvrije, op sorptie gebaseerde JT koeler voor het METIS instrument in het E-ELT project. Het onderzoek wordt gekarakteriseerd door verschillende grote uitdagingen zoals: meerdere temperatuurniveaus, maakbaarheid, kosten, een hoog koelvermogen met een hoge efficiëntie en beperkte afmetingen, enzovoort. Dit onderzoek is in de volgende stappen uitgevoerd:

\section{De initialisatie:}

De basisprincipes van de sorptiekoelertechnologie zijn verzameld en bestudeerd, evenals de belangrijkste invoerparameters (zoals de eigenschappen van het gas, karakterisatie van de absorbents en de cryogene eigenschappen van de materialen). Gebaseerd op de eigenschappen van het werkgas en de absorbent is een generieke methode ontwikkeld om het beste werkgas te selecteren voor een op sorptie gebaseerde JT koeler voor het METIS koelsysteem. Bovendien is een geavanceerde adsorptie-isothermenmeetopstelling aangepast om de nauwkeurigheid ervan te verbeteren. Met deze opstelling 
zijn een grote hoeveelheid adsorptie-isothermen gemeten. Deze isothermen dienen als input voor het ontwerp van de sorptiecompressor.

\section{Het standaard systeemontwerp:}

In de systeemontwerpfase is een conceptueel ontwerp van het koelsysteem gemaakt voor de METIS koeler. Drie parallelle koeltrappen worden thermisch aan elkaar verbonden en zorgen voor koeling bij $8 \mathrm{~K}$ (helium), $25 \mathrm{~K}$ (waterstof) en $40 \mathrm{~K}$ (neon). De sorptiecompressoren zijn thermisch verankerd op een basistemperatuur van $70 \mathrm{~K}$. Een quasistatisch model is ontwikkeld waarmee de bedrijfsparameters geoptimaliseerd zijn (zoals compressordrukken en -temperaturen), en de prestaties van het koelsysteem zijn afgeschat. Gebaseerd op het systeemontwerp is de meest kritische koelertrap gedentificeerd, namelijk de heliumtrap, en in het vervolg van het onderzoek is op deze trap dan ook de nadruk gelegd.

\section{Ontwerp en validatie van deelsystemen:}

Volgend op het conceptueel ontwerp van het METIS koelsysteem zijn twee kritische delen van het koelsysteem nader beschouwd en ontwikkeld, namelijk (a) de helium koude trap en (b) de helium sorptiecompressor.

(a) De helium JT koude trap:

Deze bestaat uit een tegenstroomwarmtewisselaar, een JT restrictie en de detectorwarmtewisselaar op een temperatuur van $8 \mathrm{~K}$. Numerieke modellen zijn ontwikkeld om deze componenten te ontwerpen en te optimaliseren. Een volledige $8 \mathrm{~K}$ helium JT koude trap is gefabriceerd en getest als prototype voor de helium JT koude trap in het METIS koelsysteem. In deze meetopstelling is een Gifford-McMahon koeler gebruikt om de voorkoeltemperaturen te realiseren en een mechanische compressor om de koude trap te voeden. De metingen gaven aan dat deze JT koude trap een koelvermogen van $0.42 \mathrm{~W}$ bij $8.0 \mathrm{~K}$ kan leveren, hetgeen boven de ontwerpeis ligt $(0.4 \mathrm{~W})$.

(b) De helium sorptiecompressor:

Een geschaalde versie van de helium-compressor is gerealiseerd om het ontwerp van de helium-sorptiecompressor van de METIS koeler te valideren, met name betreffende het rendement, in relatie tot de afmetingen van de cel en het dynamische gedrag. Een nieuw type sorptiecompressor is ontwikkeld werkend zonder een zogenaamde gasgap heat switch. Dit type gebruikt ongeveer $20 \%$ meer vermogen maar resulteert in $14 \%$ minder sorptiecellen in de METIS koeler, en is bovendien veel gemakkelijker en goedkoper te produceren. Een één-dimensionaal dynamisch model, welke het gedrag van de sorptiecompressor simuleert en de efficintie kan voorspellen, is ontwikkeld in MATLAB en experimenteel gevalideerd. Op basis hiervan is een demo-opstelling ontworpen, gefabriceerd, en getest. In het standaard ontwerp genereert een compressorcel een debiet van $2.73 \mathrm{mg} / \mathrm{s}$ bij een basistemperatuur van $70 \mathrm{~K}$. Door laagjes aluminium 
folie tussen de koolstofpillen te plaatsen wordt de warmtegeleiding in radiale richting verbeterd en wordt een 7 tot $9 \%$ hoger debiet gerealiseerd. Het één-dimensionaal dynamische sorptiemodel bleek de compressorwerking prima te kunnen simuleren. Verschillen tussen de simulaties en de experimentele resultaten, van enkele procenten, kunnen volledig verklaard worden door onzekerheden in de invoerparameters van het model zoals de sorptie-isothermen, de warmtecapaciteiten en de thermische geleiding. Ondanks de gevoeligheid voor deze invoerparameters is het model erg nuttig in de ontwerpfase. Uiteindelijk is een compressor gemaakt, werkend met drie cellen, die 120 graden uit fase lopen, welke een debiet haalt van $9.2 \mathrm{mg} / \mathrm{s}$.

\section{Demonstratie van een complete koeler:}

Een complete $40 \mathrm{~K}$ neon demo-koeler wordt momenteel ontwikkeld, samen met Airbus Defence \& Space Nederland, om de haalbaarheid van de sorptiekoeler technologie voor deze specifieke grootschalige toepassing aan te tonen. De $40 \mathrm{~K}$ JT koude trap is ontworpen, gefabriceerd en getest. Een koelvermogen van $1 \mathrm{~W}$ bij $40 \mathrm{~K}$ werd behaald, zoals verwacht. We hebben ook de neon sorptiecompressor ontworpen die momenteel wordt gefabriceerd bij Airbus Defence \& Space Nederland. Als deze sorptiecompressor af is zal deze gecombineerd worden met de koude trap voor experimenten aan een complete koeler.

\section{Outlook:}

Om sorptiekoelertechnologie nog beter te kunnen laten concurreren met traditionele mechanische koelers zullen verdere verbeteringen op het gebied van efficiëntie en afmetingen nodig zijn. Hierbij moeten ook aspecten als produceerbaarheid, complexiteit van het systeem en kosten meegenomen worden. Om dit gerealiseerd te krijgen kunnen de volgende onderwerpen beschouwd worden: het verbeteren van de hoeveelheid geadsorbeerd gas aan de adsorbent door verdere optimalisering van de micro/meso structuur en de poriegrootte verdeling van de adsorbent, het zoeken naar nieuwe adsorbents (zoals metalorganic frameworks), de ontwikkeling van een drie-dimensionaal sorptiemodel voor een nader begrip van warmte- en massatransportverschijnselen in de sorptiecompressor, het verbeteren van de efficiëntie van warmtetransport bij het opwarmen en afkoelen van de adsorbent, het verkleinen van de drukval in de sorptiecel, het optimaliseren van het opwarming van de adsorbent in de desorptie-fase, het regenereren van warmte, en het zoeken naar betere alternatieven voor de gas-gap heat switch. 



\section{Acknowledgements}

The Netherlands is the place where modern cryogenics started when Heike Kamerlingh Onnes first liquefy the helium (in 1908) and fund the superconductivity (in 1911) in his laboratory at Leiden University. This small beautiful country had been appealing to me as a Holy Land since I began my study on cryogenics. Therefore, it has been my truly privilege having such a wonderful cryogenic journey in the Netherlands. This thesis concludes the research work that I have carried out in the Energy, Materials and Systems (EMS) group of the University of Twente. With no doubt, it could not be done without the advice, help and encouragement that I have received in the past five years. Here I would like to present my acknowledgement to the people who have contributed to the successful outcome of my Ph.D. research.

First and foremost I wish to express my sincere gratitude to my advisor, Prof. Marcel ter Brake, who offered me this great opportunity to conduct my Ph.D. study in EMS. I have received his valuable advice and consistent encouragement throughout my research work. Thanks to Marcels patient guidance, I was able to concentrate my effort to the right direction at the beginning of my Ph.D. where I was at a loss facing multiple complex problems at the same time and obsessing about detail issues. Both consciously and unconsciously, Marcel has taught me to step back and think about the big picture from time to time during my research work. These systematic thinking and scientific methodologies are the most important thing that I have learned during my Ph.D. study. Furthermore, the joy and enthusiasm he has for the research was contagious and motivational for me as well. I appreciate all Marcels contributions of time, ideas and funding to make my Ph.D. experience productive and stimulating.

I would like to thank Cris Vermeer and Harry Holland for offering their solid technical support during my study. Cris helped me to fabricate and build most of the experimen- 
tal setups that described in my thesis. Lab work is full of failures and unexpectedness that kills ones will imperceptibly. But Cris always says This is not a problem but a challenge for us. His optimism has brought so much positive energy and determination into the work. With such enthusiasm, we overcame a lot of technical difficulties and had a great sense of achievement. During the lab work, we also had a lot of fun by telling jokes, discussing culture differences and sharing our traveling experiences. It was really a memorable experience. Harry, with 20-years experience on cryogenic engineering, is the living cryogenic technical Wikipedia in our lab. From Harry, I have learned a lot of empirical knowledges and technical skills that for sure will be my valuable assets for my future career.

I feel very grateful for the fellowship with Dr. Haishan Cao. Haishan and I both graduated from Zhejiang University in China. However, we had never known each other until I came to Netherlands. Haishan provided me a lot of helps and suggestions concerning my daily life when I first came to this country. At work, I have also very much enjoyed the discussions and brain storms between us related to all kind of science and technology.

I would like to appreciate Dr. David Zalewski and Dr. Johannes Burger for coaching me at the very beginning of the Ph.D. project. With their help, I was able to quickly master the fundamental knowledge of sorption-based cooling technology and basic cryogenic experimental skills. Their work on exploring adsorption characteristics on activated carbons provided essential inputs for the METIS sorption cooler development.

Thanks to the students I coached for their assistance and contributions to the project: Bram Hesselink, Bram Stapelbroek and Jeroen van Beek for measuring the adsorption isotherms, Edwin Dollekamp for his study on helium JT cold stage and Tim Mulder for his work on experimental verification of the sorption compressor model.

My sincere thanks also goes to Dr. Srinivas (Srini) Vanapalli, who has offered me plenty of useful information in respect to my postdoctoral works. Srini has shared his constructive criticism and opinions on my future career which has broaden my perspectives and given me more confidence during my job searching.

My research work has also received inputs, comments and reviews from extensive collaborations. Regarding that, I give my thanks to Prof. Bernhard Brandl at Leiden University, Dr. Frank Molster at Nederlandse Onderzoekschool Voor Astronomie, Bruin Benthem at Airbus Defence and Space Netherlands B.V., Ronald Roelfsema at Netherlands Institute for Radio Astronomy, Prof. T.H. van der Meer at the Laboratory of Thermal Engineering in University of Twente, Prof. A.T.A.M. de Waele at Eindhoven University of Technology and Jie Chen at Pengli Cryogenic Technology Inc..

My special gratitude goes to my master supervisors, Prof. Zhihua Gan and Prof. Limin Qiu, in Cryoboat group of Zhejiang University. It was their consistent effort on international collaborations that widened my horizons and consequently facilitated my oversea study. They have also given me many useful advice and suggestions regarding to my career.

EMS is a big family and full of joy. Here I would like to thank our secretaries Ans 
and Inke who kindly offered many assistance in arranging my employment, business trips and graduations. I give my thanks to Chao, Hendrie, Nir, Konstantyn, Marc, Ruben, Gabriella, Gonzalo, Wilco, Tommaso, Marcel, Peng, Anne, Afroditi, Jaap, Bas, Hidde, and Jianfeng for all the enjoyable time in this warm family and the fruitful discussions at the colloquiums.

Moreover, I have met many nice friends in Enschede. I would like to express my special thanks to Li Liu who like a big sister gave me lots of helps and suggestions to my daily life. I also thank all my friends I met on the basketball court, Xiaokui Zhang, Tianpei Wang, Zhao Wang, Zhou Fang, Wenlong Chen, Yin Tao et al. It was my honor to play and compete on the same court.

Lastly, I would like to thank my parents for all their love, encouragement and understanding. I love them so much, and I would not made it this so far without them. My beloved wife, Yi, you has been my constant source of courage and inspiration, which allowed me to finish this journey. We have been through so much together in the past five years, overcoming countless difficulties. I am truly grateful for your unconditional love and faithful support, and feel so happy and exciting about the beautiful adventure we are about to embark upon together. 



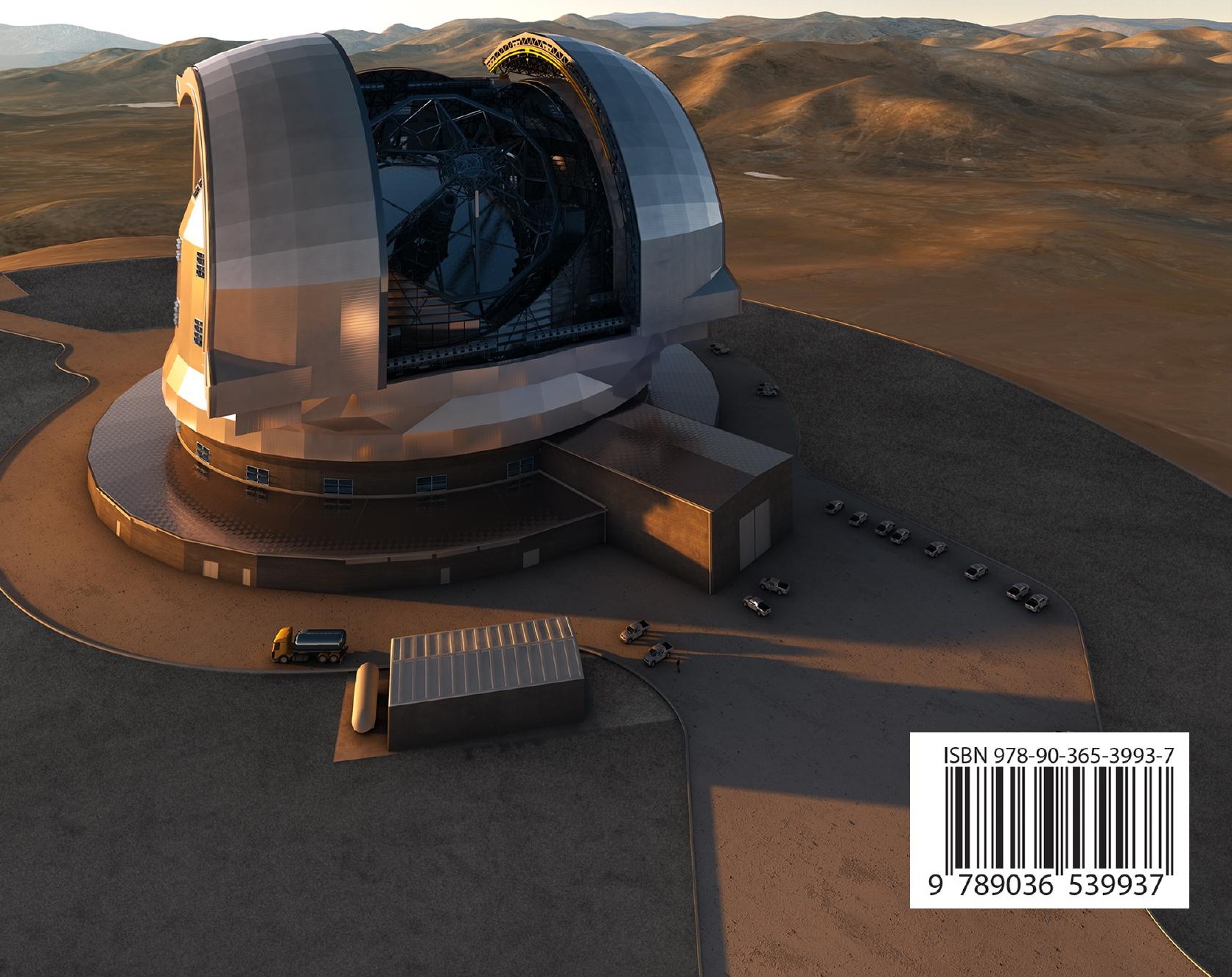

